[Supporting Information]

\title{
Difluoroisoxazolacetophenone: A Difluoroalkylation Reagent for Organocatalytic Vinylogous Nitroaldol Reactions of 1,2-Diketones
}

Yong Zhang,* Jin Ge, Liang Luo, Su-Qiong Yan, Guo-Wei Lai, Zu-Qin Mei, Hai-Qing Luo and Xiao-Lin Fan

Key Laboratory of Organo-pharmaceutical Chemistry, Gannan Normal University, Ganzhou 341000, P. R. China.

Fax:+86(0)797 8393536; E-mail: yong_zhanggnnu@126.com

\section{Contents}

1. General information S2

2. Experimental section S4

3. Crystal structure S18

4. NMR spectra S19 


\section{General information}

\section{General methods}

NMR spectra were recorded on a liquid NMR spectrometer $\left(400 \mathrm{MHz}\right.$ for ${ }^{1} \mathrm{H}$, $101 \mathrm{MHz}$ for ${ }^{13} \mathrm{C}$ and $376 \mathrm{MHz}$ for ${ }^{19} \mathrm{~F}$ ) using DMSO- $d_{6}$ as the solvent. The residual proton in DMSO- $d_{6}(\delta=2.50)$ served as an internal standard for ${ }^{1} \mathrm{H}$ NMR, and the ${ }^{13} \mathrm{C}$-atom of DMSO- $d_{6}$ was used as an internal standard $(\delta=39.52)$ for ${ }^{13} \mathrm{C}$ NMR. Chemical shifts are reported in ppm and the coupling constants $J$ are given in Hz. The following abbreviations were used to explain the multiplicities: s (singlet), d (doublet), $\mathrm{t}$ (triplet), q (quartet), m (multiplet), br (broad). HRMS anaylses were performed at Bruker Daltonic. Inc mass instrument (ESI) and Thermo Scientific LTQ-Orbitrap XL.

\section{Materials}

Isatins were commercially available. $\mathrm{N}$-protected isatins were prepared according to the literature procedures. ${ }^{1}$ Difluoroisoxazolacetophenone $\mathbf{2}$ and monofluoroisoxazolacetophenone $\mathbf{6}$ were prepared according to the followed procedures. Unless otherwise noted, analytical grade solvents and commercially available reagents were used without further purification.

\section{Experimental procedure for the synthesis of difluoroisoxazolacetophenone 2a}

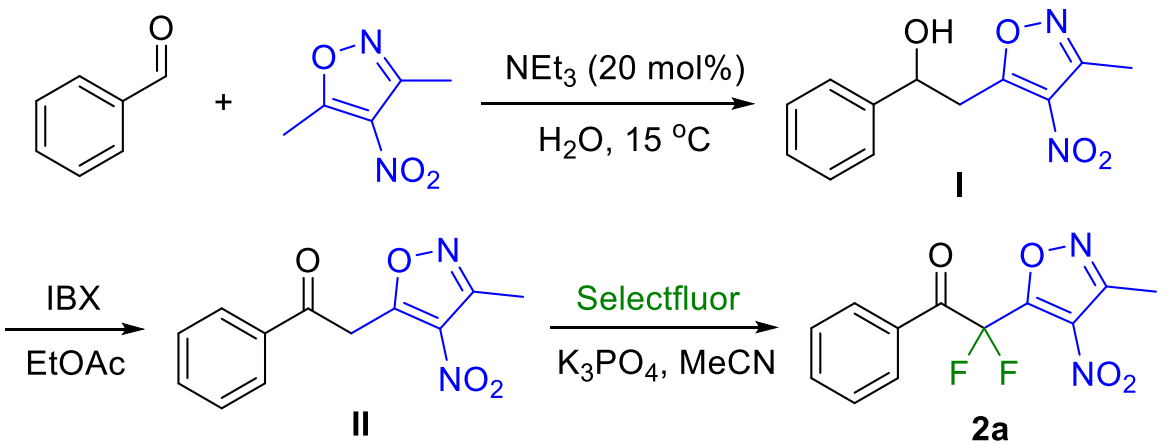

The mixture of 3,5-dimethyl-4-nitroisoxazole (1.42 g, $10.0 \mathrm{mmol})$, benzaldehyde $(1.17 \mathrm{~g}, 11.0 \mathrm{mmol})$, triethylamine $(2.0 \mathrm{mmol})$ in $5 \mathrm{~mL} \mathrm{H}_{2} \mathrm{O}$ were stirred at room temperature for 16 hours. After the reaction was completed (according to the TLC analysis), the mixture was extracted with ethyl acetate. The combined organic phase was dried over $\mathrm{Na}_{2} \mathrm{SO}_{4}$ and concentrated in vacuo. The residue was subjected to silica gel chromatography using PE/EA (v/v = 10/1) as eluent to afford I in $90 \%$ yield $(2.23$ g).

A mixture of $\mathbf{I}(1.0 \mathrm{mmol})$ and $o$-iodoxybenzoic acid (IBX) $(2.0 \mathrm{mmol})$ in EtOAc $(10 \mathrm{~mL})$ was refluxed at $80{ }^{\circ} \mathrm{C}$ (oil bath) for $6 \mathrm{~h}$. After completion of the reaction (monitored by TLC), the reaction mixture was cooled to room temperature and filtered. The filtrate was concentrated and recrystallized from ethanol to give II in $88 \%$ 
yield (216.5 mg).

II $(0.739 \mathrm{~g}, 3.0 \mathrm{mmol})$, Selectfluor $(2.34 \mathrm{~g}, 6.6 \mathrm{mmol})$ and potassium phosphate $(0.669 \mathrm{~g}, 3.15 \mathrm{mmol})$ were dissolved in $6.6 \mathrm{~mL}$ of anhydrous acetonitrile. The mixture was stirred at room temperature for 1 day and the reaction was monitored by TLC analysis. Once completed, the reaction mixture was filtered and washed several times with dichloromethane. The combined organic layers were washed with saturated aqueous ammonium chloride and dried over anhydrous $\mathrm{Na}_{2} \mathrm{SO}_{4}$. The solvent was removed under reduced pressure to give difluoroisoxazolacetophenone 2a: pale yellow solid; mp: 99-101 ${ }^{\circ} \mathrm{C}$; $804 \mathrm{mg}, 95 \%$ yield, ${ }^{1} \mathrm{H}$ NMR (400 MHz, DMSO- $\left.d_{6}\right) \delta$ (ppm) 8.13-8.10 (m, 2H), 7.75-7.71 (m, 1H), 7.59-7.55 (m, 2H), $2.65(\mathrm{~s}, 3 \mathrm{H}) ;{ }^{13} \mathrm{C}$ NMR (101 MHz, DMSO- $\left.d_{6}\right) \delta(\mathrm{ppm}) 184.5\left(\mathrm{t}, J_{\mathrm{C}-\mathrm{F}}=28.6 \mathrm{~Hz}, 1 \mathrm{C}\right), 162.0\left(\mathrm{t}, J_{\mathrm{C}-\mathrm{F}}=\right.$ $30.0 \mathrm{~Hz}, 1 \mathrm{C}), 156.5,135.2,130.7\left(\mathrm{t}, J_{\mathrm{C}-\mathrm{F}}=2.7 \mathrm{~Hz}, 1 \mathrm{C}\right), 130.0\left(\mathrm{t}, J_{\mathrm{C}-\mathrm{F}}=2.6 \mathrm{~Hz}, 1 \mathrm{C}\right)$, 129.0, $111.9\left(\mathrm{t}, J_{\mathrm{C}-\mathrm{F}}=261.4 \mathrm{~Hz}, 1 \mathrm{C}\right), 11.0 .{ }^{19} \mathrm{~F}$ NMR (376 MHz, DMSO- $\left.d_{6}\right) \delta(\mathrm{ppm})$ -99.8 (s, 2F). HRMS (ESI) m/z: [M - H]- Calcd for $\mathrm{C}_{12} \mathrm{H}_{7} \mathrm{~F}_{2} \mathrm{~N}_{2} \mathrm{O}_{4} 281.0379$; Found 281.0379 .

\section{Experimental procedure for the synthesis of monofluoroisoxazolacetophenone 6}

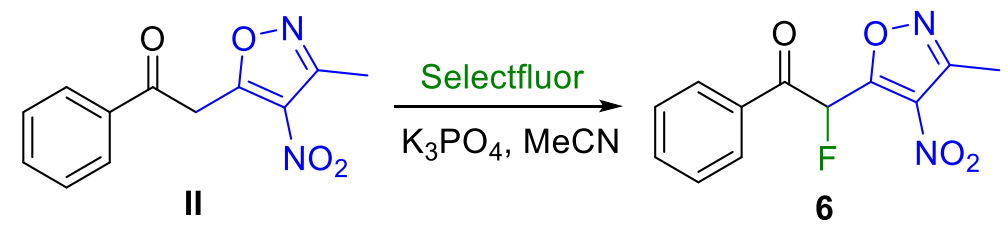

II (0.246 g, $1.0 \mathrm{mmol})$, Selectfluor $(0.354 \mathrm{mg}, 1.0 \mathrm{mmol})$ and potassium phosphate $(0.071 \mathrm{~g}, 0.33 \mathrm{mmol})$ were dissolved in $2 \mathrm{~mL}$ of anhydrous acetonitrile. The mixture was stirred at room temperature for 2 days and the reaction was monitored by TLC analysis. Once completed, the reaction mixture was filtered and washed several times with dichloromethane. The combined organic layers were washed with saturated aqueous ammonium chloride and dried over anhydrous $\mathrm{Na}_{2} \mathrm{SO}_{4}$. The solvent was removed under reduced pressure and the residue was purified by silica gel chromatography using $\mathrm{PE} /$ acetone $(\mathrm{v} / \mathrm{v}=15 / 1)$ as eluent to give monofluoroisoxazolacetophenone (6) : pale yellow liquid; $237.2 \mathrm{mg}, 90 \%$ yield, ${ }^{1} \mathrm{H}$ NMR (400 MHz, $\left.\mathrm{CDCl}_{3}\right) \delta(\mathrm{ppm}) 7.94(\mathrm{~d}, J=7.6 \mathrm{~Hz}, 2 \mathrm{H}), 7.59$ (t, $\left.J=7.4 \mathrm{~Hz}, 1 \mathrm{H}\right)$, $7.45(\mathrm{t}, J=7.4 \mathrm{~Hz}, 2 \mathrm{H}), 7.16(\mathrm{~d}, J=45.6 \mathrm{~Hz}, 1 \mathrm{H}), 2.52(\mathrm{~s}, 3 \mathrm{H}) ;{ }^{13} \mathrm{C} \mathrm{NMR}(101 \mathrm{MHz}$, $\left.\mathrm{CDCl}_{3}\right) \delta(\mathrm{ppm}) 188.6\left(\mathrm{~d}, J_{\mathrm{C}-\mathrm{F}}=20.9 \mathrm{~Hz}, 1 \mathrm{C}\right), 165.2\left(\mathrm{t}, J_{\mathrm{C}-\mathrm{F}}=20.3 \mathrm{~Hz}, 1 \mathrm{C}\right), 155.7$, $134.8,133.4,133.1\left(\mathrm{~d}, J_{\mathrm{C}-\mathrm{F}}=2.1 \mathrm{~Hz}, 1 \mathrm{C}\right), 129.9,129.12\left(\mathrm{~d}, J_{\mathrm{C}-\mathrm{F}}=163.5 \mathrm{~Hz}, 1 \mathrm{C}\right)$, $129.08\left(\mathrm{~d}, J_{\mathrm{C}-\mathrm{F}}=3.4 \mathrm{~Hz}, 1 \mathrm{C}\right), 129.0,85.2\left(\mathrm{~d}, J_{\mathrm{C}-\mathrm{F}}=191.0 \mathrm{~Hz}, 1 \mathrm{C}\right), 11.1 .{ }^{19} \mathrm{~F}$ NMR (376 MHz, DMSO- $d_{6}$ ) $\delta$ (ppm) -189.1 (s, 1F). HRMS (ESI) m/z: [M - H]- Calcd for $\mathrm{C}_{12} \mathrm{H}_{8} \mathrm{FN}_{2} \mathrm{O}_{4}$ 263.0474; Found 263.0472.

1. (a) Dhara, K.; Mandal, T.; Das, I.; Dash, J. Angew. Chem. Int. Ed. 2015, 54, 15831-15835. (b) Ji, H. H; Zhu, Y. Z; Shao, Y; Liu. J; Yuan, Y; Jia, X. D. J. Org. Chem. 2017, 82, 9859-9865. (c) Lee, Y. C; Patil, S. S.; Golz, C.; Strohman, C.; Ziegler, S.; Kumar, K.; Waldmann, H. Nat. Commun. 2016, 8, 14043. 


\section{Experimental section}

\subsection{Attempt on organocatalyzed asymmetric vinylogous nitroaldol reaction}

Isatin 1a (14.7 mg, $0.1 \mathrm{mmol})$, difluoroisoxazolacetophenone 2a (42.3 $\mathrm{mg}, 0.15$ mmol) and $0.5 \mathrm{~mL} \mathrm{CH} \mathrm{CH}_{3} \mathrm{OH}$ were stirred at room temperature, then chiral organocatalyst $(10 \mathrm{~mol} \%, 0.01 \mathrm{mmol})$ was added. The reaction mixture was stirred for 2 days. Once completed, the reaction mixture was concentrated and purified by silica gel chromatography using $\mathrm{PE} /$ acetone $(\mathrm{v} / \mathrm{v}=3 / 1)$ as eluent. The ee value were determined by HPLC analysis using Daicel chiral AD-H column, solvent: hexane/ isopropanol $=90 / 10$, flow rate: $1.0 \mathrm{~mL} / \mathrm{min}$, detection at $254 \mathrm{~nm}$.
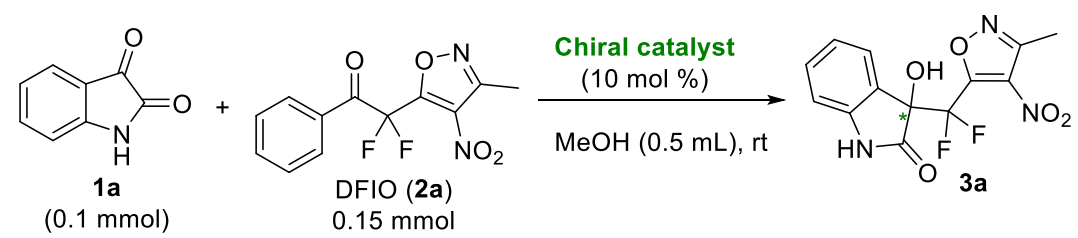

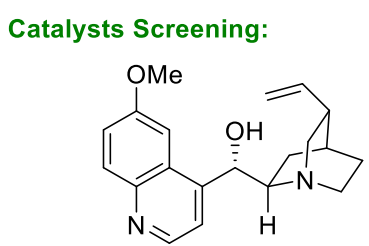

$88 \%$ yield, $0 \%$ ee

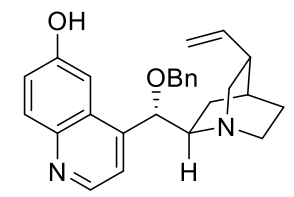

$45 \%$ yield, $0 \%$ ee

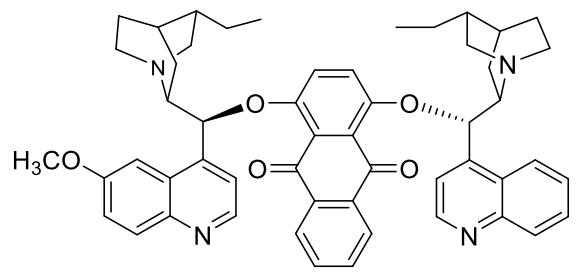

$40 \%$ yield, $0 \%$ ee

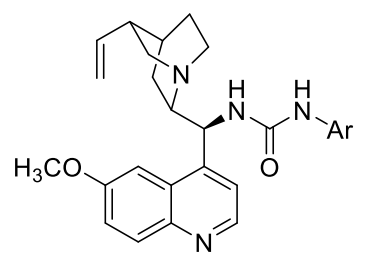

$35 \%$ yield, $0 \%$ ee $\mathrm{Ar}=3,3-\left(\mathrm{CF}_{3}\right)_{2} \mathrm{C}_{6} \mathrm{H}_{3}$

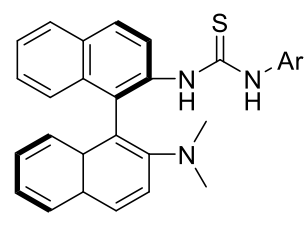

$40 \%$ yield, $0 \%$ ee

\subsection{Unsuccessful examples of vinylogous nitroaldol reaction of other electrophiles} and $2 \mathrm{a}$

Scheme S1. Unsuccessful examples

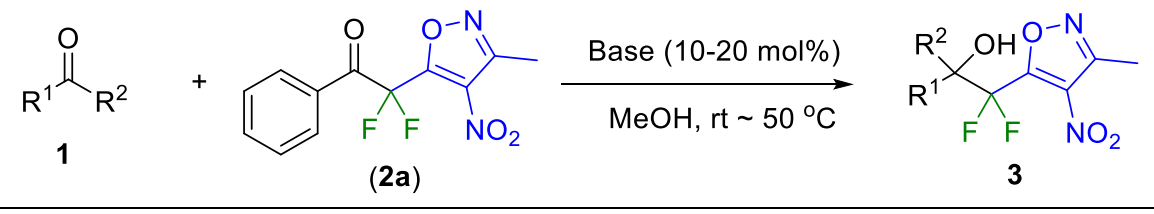

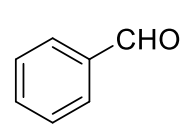

trace<smiles>COC(=O)C(=O)c1ccccc1</smiles>

no reaction<smiles>O=CC=Cc1ccccc1</smiles>

mixture<smiles>O=C(c1ccccc1)C(F)(F)F</smiles>

no reaction

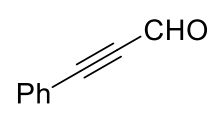

mixture<smiles>O=C(C#Cc1ccccc1)C(F)(F)F</smiles>

no reaction<smiles>CC(=O)c1ccccc1</smiles>

no reaction<smiles>O=C(C(=O)c1ccccc1)c1ccccc1</smiles>

no reaction 
2.3 Representative procedure for the direct vinylogous nitroaldol addition of difluoroisoxazolacetophenone to isatins

Isatin 1a $(0.15 \mathrm{mmol})$, difluoroisoxazolacetophenone $\mathbf{2 a}(0.20 \mathrm{mmol})$ and 0.75 $\mathrm{mL} \mathrm{CH} \mathrm{CH}_{3} \mathrm{OH}$ were stirred at room temperature, then TEA (10 mol\%, $\left.0.015 \mathrm{mmol}\right)$ was added. After the reaction was completed (indicated by color change from red to light yellow), the product 3a was purified by silica gel chromatography and isolated in high yield (eluent: PE/acetone $=3 / 1, \mathrm{v} / \mathrm{v}$ ).

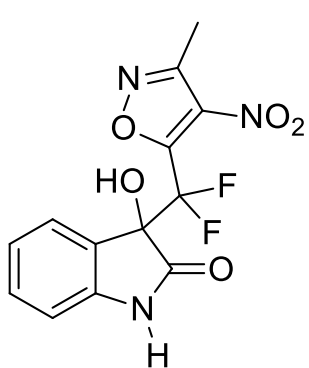

3-(Difluoro(3-methyl-4-nitroisoxazol-5-yl)methyl)-3-hydroxy-

5-methylindolin-2-one (3a): pale yellow solid; mp: 179-180 ${ }^{\circ} \mathrm{C}$;

$48.3 \mathrm{mg}, 99 \%$ yield, ${ }^{1} \mathrm{H}$ NMR (400 MHz, DMSO- $\left.d_{6}\right) \delta(\mathrm{ppm})$ $10.82(\mathrm{~s}, 1 \mathrm{H}), 7.59$ (s, 1H), 7.39 (dd, $J=18.4,7.6 \mathrm{~Hz}, 2 \mathrm{H}), 7.08$ $(\mathrm{t}, J=7.4 \mathrm{~Hz}, 1 \mathrm{H}), 6.92(\mathrm{~d}, J=8.0 \mathrm{~Hz}, 1 \mathrm{H}), 2.53(\mathrm{~s}, 3 \mathrm{H}) ;{ }^{13} \mathrm{C}$ NMR (101 MHz, DMSO- $\left.d_{6}\right) \delta(\mathrm{ppm}) 172.7\left(\mathrm{~d}, J_{\mathrm{C}-\mathrm{F}}=6.3 \mathrm{~Hz}, 1 \mathrm{C}\right)$, $157.8\left(\mathrm{t}, J_{\mathrm{C}-\mathrm{F}}=33.7 \mathrm{~Hz}, 1 \mathrm{C}\right), 156.7,143.0,133.3,131.4,126.5$, $124.7,122.4,115.7\left(\mathrm{dd}, J_{\mathrm{C}-\mathrm{F}}=255.3,249.8 \mathrm{~Hz}, 1 \mathrm{C}\right), 110.4,77.2$ $\left(\mathrm{dd}, J_{\mathrm{C}-\mathrm{F}}=31.6,24.6 \mathrm{~Hz}, 1 \mathrm{C}\right), 10.8 .{ }^{19} \mathrm{~F}$ NMR $\left(376 \mathrm{MHz}, \mathrm{DMSO}-d_{6}\right) \delta(\mathrm{ppm})-106.7$ $(\mathrm{d}, J=276.0 \mathrm{~Hz}, 1 \mathrm{~F}),-108.5$ (d, $J=276.0 \mathrm{~Hz}, 1 \mathrm{~F})$. HRMS (ESI) m/z: [M - H]- Calcd for $\mathrm{C}_{13} \mathrm{H}_{8} \mathrm{~F}_{2} \mathrm{~N}_{3} \mathrm{O}_{5} 324.0438$; Found 324.0437 .

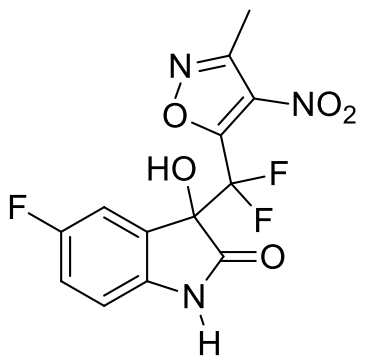

3-(Difluoro(3-methyl-4-nitroisoxazol-5-yl)methyl)-5-fluoro

-3-hydroxyindolin-2-one (3b): yellow solid; $\mathrm{mp}$ : decomposed at $201{ }^{\circ} \mathrm{C} ; 50.9 \mathrm{mg}, 99 \%$ yield, ${ }^{1} \mathrm{H}$ NMR (400 $\mathrm{MHz}$, DMSO- $\left.d_{6}\right) \delta(\mathrm{ppm}) 10.88(\mathrm{~s}, 1 \mathrm{H}), 7.77(\mathrm{~s}, 1 \mathrm{H})$, 7.28-7.22 (m, 2H), $6.94(\mathrm{dd}, J=8.0,4.0 \mathrm{~Hz}, 1 \mathrm{H}), 2.53(\mathrm{~s}, 3 \mathrm{H})$; ${ }^{13} \mathrm{C}$ NMR (101 MHz, DMSO- $\left.d_{6}\right) \delta(\mathrm{ppm}) 172.5\left(\mathrm{~d}, J_{\mathrm{C}-\mathrm{F}}=6.2\right.$ $\mathrm{Hz}, 1 \mathrm{C}), 158.0\left(\mathrm{~d}, J_{\mathrm{C}-\mathrm{F}}=237.2 \mathrm{~Hz}, 1 \mathrm{C}\right), 157.5\left(\mathrm{t}, J_{\mathrm{C}-\mathrm{F}}=33.7\right.$

$\mathrm{Hz}, 1 \mathrm{C}), 156.8,139.2\left(\mathrm{~d}, J_{\mathrm{C}-\mathrm{F}}=1.7 \mathrm{~Hz}, 1 \mathrm{C}\right), 133.4,126.1\left(\mathrm{~d}, J_{\mathrm{C}-\mathrm{F}}=8.1 \mathrm{~Hz}, 1 \mathrm{C}\right), 117.9$ $\left(\mathrm{d}, J_{\mathrm{C}-\mathrm{F}}=23.2 \mathrm{~Hz}, 1 \mathrm{C}\right), 115.5\left(\mathrm{dd}, J_{\mathrm{C}-\mathrm{F}}=255.7,249.7 \mathrm{~Hz}, 1 \mathrm{C}\right), 114.1\left(\mathrm{~d}, J_{\mathrm{C}-\mathrm{F}}=25.0\right.$ $\mathrm{Hz}, 1 \mathrm{C}), 111.5\left(\mathrm{~d}, J_{\mathrm{C}-\mathrm{F}}=7.8 \mathrm{~Hz}, 1 \mathrm{C}\right), 77.4\left(\mathrm{dd}, J_{\mathrm{C}-\mathrm{F}}=31.5,24.9 \mathrm{~Hz}, 1 \mathrm{C}\right), 10.8 .{ }^{19} \mathrm{~F}$ NMR (376 MHz, DMSO- $\left.d_{6}\right) \delta(\mathrm{ppm})-106.9(\mathrm{~d}, J=276.4 \mathrm{~Hz}, 1 \mathrm{~F}),-108.6(\mathrm{~d}, J=$ $276.7 \mathrm{~Hz}, 1 \mathrm{~F}),-120.6(\mathrm{~s}, 1 \mathrm{~F})$. HRMS (ESI) m/z: [M - H]- Calcd for $\mathrm{C}_{13} \mathrm{H}_{7} \mathrm{~F}_{3} \mathrm{~N}_{3} \mathrm{O}_{5}$ 342.0343; Found 342.0341.

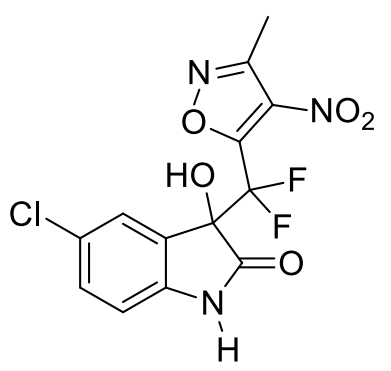

\section{5-Chloro-3-(difluoro(3-methyl-4-nitroisoxazol-5-yl)methyl}

)- 3-hydroxyindolin-2-one (3c): yellow solid; mp: decomposed at $208{ }^{\circ} \mathrm{C} ; 49.1 \mathrm{mg}, 91 \%$ yield, ${ }^{1} \mathrm{H}$ NMR (400 $\left.\mathrm{MHz}, \mathrm{DMSO}-d_{6}\right) \delta(\mathrm{ppm}) 11.03(\mathrm{~s}, 1 \mathrm{H}), 7.83(\mathrm{~s}, 1 \mathrm{H})$, 7.46-7.43 (m, 2H), $6.96(\mathrm{~d}, J=8.4 \mathrm{~Hz} 1 \mathrm{H}), 2.53(\mathrm{~s}, 3 \mathrm{H}) ;{ }^{13} \mathrm{C}$ NMR (101 MHz, DMSO- $\left.d_{6}\right) \delta(\mathrm{ppm}) 172.4\left(\mathrm{~d}, J_{\mathrm{C}-\mathrm{F}}=6.5 \mathrm{~Hz}\right.$, $1 \mathrm{C}), 157.5\left(\mathrm{t}, J_{\mathrm{C}-\mathrm{F}}=33.7 \mathrm{~Hz}, 1 \mathrm{C}\right), 156.9,142.0,133.5,131.4$, $126.5,126.5,115.6\left(\mathrm{dd}, J_{\mathrm{C}-\mathrm{F}}=255.8,249.7 \mathrm{~Hz}, 1 \mathrm{C}\right), 112.2,77.3\left(\mathrm{dd}, J_{\mathrm{C}-\mathrm{F}}=31.8,24.4\right.$ $\mathrm{Hz}, 1 \mathrm{C}), 10.8 .{ }^{19} \mathrm{~F}$ NMR (376 MHz, DMSO- $\left.d_{6}\right) \delta(\mathrm{ppm})-106.8(\mathrm{~d}, J=277.1 \mathrm{~Hz}, 1 \mathrm{~F})$, 
$-108.5(\mathrm{~d}, J=276.7 \mathrm{~Hz}, 1 \mathrm{~F})$. HRMS (ESI) m/z: [M - H]- Calcd for $\mathrm{C}_{13} \mathrm{H}_{7} \mathrm{ClF}_{2} \mathrm{~N}_{3} \mathrm{O}_{5}$ 358.0048 ; Found 358.0049.

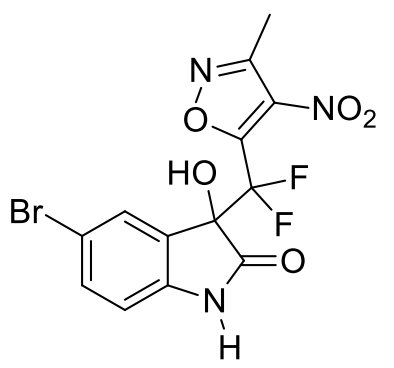

5-Bromo-3-(difluoro(3-methyl-4-nitroisoxazol-5-yl)methyl )-3-hydroxyindolin-2-one (3d): pale yellow solid; $\mathrm{mp}$ : decomposed at $205{ }^{\circ} \mathrm{C} ; 50.8 \mathrm{mg}, 84 \%$ yield, ${ }^{1} \mathrm{H}$ NMR $(400$ MHz, DMSO- $\left.d_{6}\right) \delta(\mathrm{ppm}) 11.02(\mathrm{~s}, 1 \mathrm{H}), 7.79(\mathrm{~s}, 1 \mathrm{H}), 7.59(\mathrm{~d}$, $J=8.4 \mathrm{~Hz}, 1 \mathrm{H}), 7.54(\mathrm{~s}, 1 \mathrm{H}), 6.91(\mathrm{~d}, J=8.4 \mathrm{~Hz}, 1 \mathrm{H}), 2.53$ $(\mathrm{s}, 3 \mathrm{H}) ;{ }^{13} \mathrm{C}$ NMR (101 MHz, DMSO- $\left.d_{6}\right) \delta(\mathrm{ppm}) 172.1(\mathrm{~d}$, $\left.J_{\mathrm{C}-\mathrm{F}}=6.5 \mathrm{~Hz}, 1 \mathrm{C}\right), 157.4\left(\mathrm{t}, J_{\mathrm{C}-\mathrm{F}}=33.8 \mathrm{~Hz}, 1 \mathrm{C}\right), 156.8,142.3$, 134.1, 133.4, 129.1, 126.8, 115.5 (dd, $\left.J_{\mathrm{C}-\mathrm{F}}=255.9,250.0 \mathrm{~Hz}, 1 \mathrm{C}\right), 113.9,112.6,77.2$ $\left(\mathrm{dd}, J_{\mathrm{C}-\mathrm{F}}=31.9,24.5 \mathrm{~Hz}, 1 \mathrm{C}\right), 10.8 .{ }^{19} \mathrm{~F}$ NMR $\left(376 \mathrm{MHz}, \mathrm{DMSO}-d_{6}\right) \delta(\mathrm{ppm})-106.6$ (d, $J=276.7 \mathrm{~Hz}, 1 \mathrm{~F}),-108.5$ (d, $J=276.7 \mathrm{~Hz}, 1 \mathrm{~F})$. HRMS (ESI) m/z: [M - H]- Calcd for $\mathrm{C}_{13} \mathrm{H}_{7} \mathrm{BrF}_{2} \mathrm{~N}_{3} \mathrm{O}_{5} 401.9543$; Found 401.9538 .

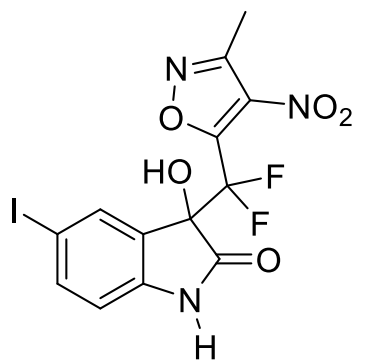

\section{3-(Difluoro(3-methyl-4-nitroisoxazol-5-yl)methyl)-3-hydro} xy-5-iodoindolin-2-one (3e): yellow solid; mp: decomposed at $206{ }^{\circ} \mathrm{C} ; 64.3 \mathrm{mg}, 95 \%$ yield, ${ }^{1} \mathrm{H}$ NMR (400 MHz, DMSO- $d_{6}$ ) $\delta(\mathrm{ppm}) 10.99(\mathrm{~s}, 1 \mathrm{H}), 7.74(\mathrm{~m}, 2 \mathrm{H}), 7.67(\mathrm{~s}, 1 \mathrm{H}), 6.79(\mathrm{~d}, J=$ $8.4 \mathrm{~Hz}, 1 \mathrm{H}), 2.53(\mathrm{~s}, 3 \mathrm{H}) ;{ }^{13} \mathrm{C}$ NMR $\left(101 \mathrm{MHz}, \mathrm{DMSO}-d_{6}\right) \delta$ $(\mathrm{ppm}) 171.9\left(\mathrm{~d}, J_{\mathrm{C}-\mathrm{F}}=6.5 \mathrm{~Hz}, 1 \mathrm{C}\right), 157.4\left(\mathrm{t}, J_{\mathrm{C}-\mathrm{F}}=33.7 \mathrm{~Hz}\right.$, 1C), $156.7,142.7,139.8,134.5,133.3,127.0,115.5\left(\mathrm{dd}, J_{\mathrm{C}-\mathrm{F}}=\right.$ $255.5,249.6 \mathrm{~Hz}, 1 \mathrm{C}), 112.9,85.0,77.0\left(\mathrm{dd}, J_{\mathrm{C}-\mathrm{F}}=31.9,24.4 \mathrm{~Hz}, 1 \mathrm{C}\right), 10.7 .{ }^{19} \mathrm{~F}$ NMR $\left(376 \mathrm{MHz}, \mathrm{DMSO}-d_{6}\right) \delta(\mathrm{ppm})-106.6(\mathrm{~d}, J=276.4 \mathrm{~Hz}, 1 \mathrm{~F}),-108.6(\mathrm{~d}, J=277.1 \mathrm{~Hz}$, 1F). HRMS (ESI) m/z: [M - H]- Calcd for $\mathrm{C}_{13} \mathrm{H}_{7} \mathrm{~F}_{2} \mathrm{IN}_{3} \mathrm{O}_{5} 449.9404$; Found 449.9401.

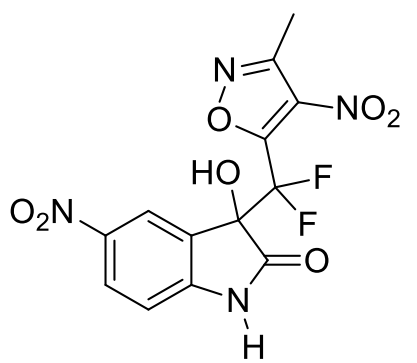

3-(Difluoro(3-methyl-4-nitroisoxazol-5-yl)methyl)-3-hyd roxy-5-nitroindolin-2-one (3f): yellow solid; mp: 149-151 ${ }^{\circ} \mathrm{C} ; 45.0 \mathrm{mg}, 81 \%$ yield, ${ }^{1} \mathrm{H}$ NMR (400 MHz, DMSO- $\left.d_{6}\right) \delta$ (ppm) $11.62(\mathrm{~s}, 1 \mathrm{H}), 8.36(\mathrm{dd}, J=8.8,2.4 \mathrm{~Hz}, 1 \mathrm{H}), 8.23$ (s, $1 \mathrm{H}), 8.06(\mathrm{~s}, 1 \mathrm{H}) 7.17(\mathrm{~d}, J=8.8 \mathrm{~Hz}, 1 \mathrm{H}), 2.54(\mathrm{~s}, 3 \mathrm{H}) ;{ }^{13} \mathrm{C}$ NMR $\left(101 \mathrm{MHz}, \mathrm{DMSO}-d_{6}\right) \delta(\mathrm{ppm}) 172.8\left(\mathrm{~d}, J_{\mathrm{C}-\mathrm{F}}=6.3\right.$ $\mathrm{Hz}, 1 \mathrm{C}), 157.1$ (t, $\left.J_{\mathrm{C}-\mathrm{F}}=33.6 \mathrm{~Hz}, 1 \mathrm{C}\right), 156.8,149.2,142.6$, 133.4, 128.4, 125.3, 121.9, $115.3\left(\mathrm{dd}, J_{\mathrm{C}-\mathrm{F}}=256.2,250.3 \mathrm{~Hz}, 1 \mathrm{C}\right), 110.0,76.9(\mathrm{dd}$, $\left.J_{\mathrm{C}-\mathrm{F}}=31.7,24.7 \mathrm{~Hz}, 1 \mathrm{C}\right), 10.7 .{ }^{19} \mathrm{~F}$ NMR $\left(376 \mathrm{MHz}, \mathrm{DMSO}-d_{6}\right) \delta(\mathrm{ppm})-106.6(\mathrm{~d}, J$ $=278.2 \mathrm{~Hz}, 1 \mathrm{~F}),-108.5(\mathrm{~d}, J=278.2 \mathrm{~Hz}, 1 \mathrm{~F})$. HRMS (ESI) m/z: [M - H]- Calcd for $\mathrm{C}_{13} \mathrm{H}_{7} \mathrm{~F}_{2} \mathrm{~N}_{4} \mathrm{O}_{7} 369.0288$; Found 369.0285.

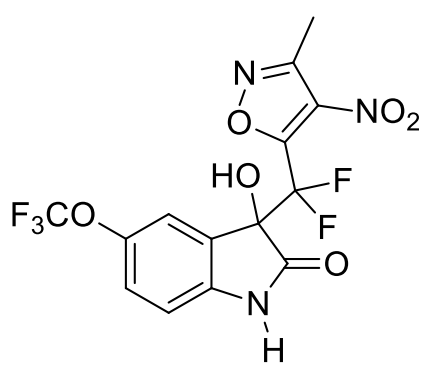

3-(Difluoro(3-methyl-4-nitroisoxazol-5-yl)methyl)-3-hy droxy-5-(trifluoromethoxy)indolin-2-one (3g): yellow solid; mp: $183-186{ }^{\circ} \mathrm{C} ; 58.3 \mathrm{mg}, 95 \%$ yield, ${ }^{1} \mathrm{H}$ NMR (400 MHz, DMSO- $\left.d_{6}\right) \delta(\mathrm{ppm}) 11.07(\mathrm{~s}, 1 \mathrm{H}), 7.86(\mathrm{~d}, J=1.6$ $\mathrm{Hz}, 1 \mathrm{H}), 7.42$ (d, $J=8.4 \mathrm{~Hz}, 1 \mathrm{H}), 7.37$ (s, 1H), 7.03 (d, $J$ $=8.4 \mathrm{~Hz}, 1 \mathrm{H}), 2.53(\mathrm{~s}, 3 \mathrm{H}) ;{ }^{13} \mathrm{C} \mathrm{NMR}(101 \mathrm{MHz}$, 
DMSO- $\left.d_{6}\right) \delta(\mathrm{ppm}) 172.5\left(\mathrm{~d}, J_{\mathrm{C}-\mathrm{F}}=6.2 \mathrm{~Hz}, 1 \mathrm{C}\right), 157.3\left(\mathrm{t}, J_{\mathrm{C}-\mathrm{F}}=33.7 \mathrm{~Hz}, 1 \mathrm{C}\right), 156.8$, $143.4\left(\mathrm{~d}, J_{\mathrm{C}-\mathrm{F}}=1.9 \mathrm{~Hz}, 1 \mathrm{C}\right), 142.2,133.4,126.1,124.0,120.2\left(\mathrm{q}, J_{\mathrm{C}-\mathrm{F}}=254.3 \mathrm{~Hz}\right.$, $1 \mathrm{C}), 120.0,115.5\left(\mathrm{dd}, J_{\mathrm{C}-\mathrm{F}}=255.7,250.0 \mathrm{~Hz}, 1 \mathrm{C}\right), 111.7,77.3\left(\mathrm{dd}, J_{\mathrm{C}-\mathrm{F}}=31.7,24.6\right.$ $\mathrm{Hz}, 1 \mathrm{C}), 10.8 .{ }^{19} \mathrm{~F}$ NMR (376 MHz, DMSO-d $) \delta(\mathrm{ppm})-57.4$ (s, 3F), -106.9 (d, $J=$ $277.1 \mathrm{~Hz}, 1 \mathrm{~F}),-108.7$ (d, $J=276.7 \mathrm{~Hz}, 1 \mathrm{~F})$. HRMS (ESI) m/z: [M - H]- Calcd for $\mathrm{C}_{14} \mathrm{H}_{7} \mathrm{~F}_{5} \mathrm{~N}_{3} \mathrm{O}_{6} 408.0260$; Found 408.0257.

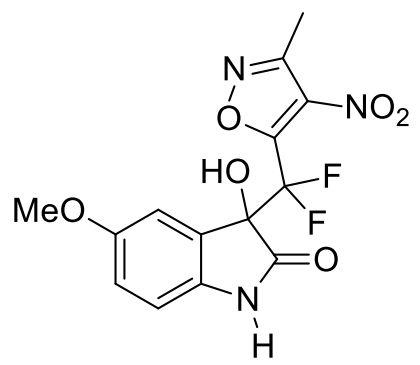

3-(Difluoro(3-methyl-4-nitroisoxazol-5-yl)methyl)-3-hyd roxy-5-methoxyindolin-2-one (3h): brown solid; $\mathrm{mp}$ : $187-188{ }^{\circ} \mathrm{C}$; $43.2 \mathrm{mg}, 81 \%$ yield, ${ }^{1} \mathrm{H}$ NMR (400 MHz, DMSO- $\left.d_{6}\right) \delta(\mathrm{ppm}) 10.65(\mathrm{~s}, 1 \mathrm{H}), 7.61(\mathrm{~s}, 1 \mathrm{H}), 6.99-6.95$ $(\mathrm{m}, 2 \mathrm{H}), 6.85-6.83(\mathrm{~m}, 1 \mathrm{H}), 3.74(\mathrm{~s}, 3 \mathrm{H}), 2.53(\mathrm{~s}, 3 \mathrm{H}) ;{ }^{13} \mathrm{C}$ NMR (101 MHz, DMSO- $\left.d_{6}\right) \delta(\mathrm{ppm}) 172.4\left(\mathrm{~d}, J_{\mathrm{C}-\mathrm{F}}=6.1\right.$ $\mathrm{Hz}, 1 \mathrm{C}), 157.7$ (t, $\left.J_{\mathrm{C}-\mathrm{F}}=33.9 \mathrm{~Hz}, 1 \mathrm{C}\right), 156.7,155.1,136.1$, $133.3,125.7,116.1,115.7\left(\mathrm{dd}, J_{\mathrm{C}-\mathrm{F}}=255.6,250.0 \mathrm{~Hz}, 1 \mathrm{C}\right), 113.1,111.0,77.5(\mathrm{dd}$, $\left.J_{\mathrm{C}-\mathrm{F}}=31.4,24.6 \mathrm{~Hz}, 1 \mathrm{C}\right), 55.6,10.7 .{ }^{19} \mathrm{~F} \mathrm{NMR}\left(376 \mathrm{MHz}, \mathrm{DMSO}-d_{6}\right) \delta(\mathrm{ppm})$ $-107.1(\mathrm{~d}, J=276.0 \mathrm{~Hz}, 1 \mathrm{~F}),-108.6(\mathrm{~d}, J=276.0 \mathrm{~Hz}, 1 \mathrm{~F})$. HRMS (ESI) m/z: [M - H]Calcd for $\mathrm{C}_{14} \mathrm{H}_{10} \mathrm{~F}_{2} \mathrm{~N}_{3} \mathrm{O}_{6} 354.0543$; Found 354.0543 .

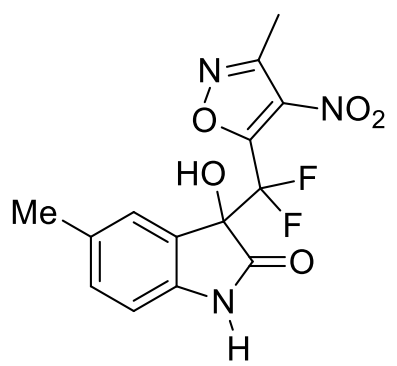

\section{3-(Difluoro(3-methyl-4-nitroisoxazol-5-yl)methyl)-3-hydr} oxy-5-methylindolin-2-one (3i): yellow solid; mp: decomposed at $192{ }^{\circ} \mathrm{C} ; 50.3 \mathrm{mg}, 99 \%$ yield, ${ }^{1} \mathrm{H}$ NMR (400 MHz, DMSO- $\left.d_{6}\right) \delta(\mathrm{ppm}) 10.71(\mathrm{~s}, 1 \mathrm{H}), 7.53(\mathrm{~s}, 1 \mathrm{H}), 7.25(\mathrm{~s}$, $1 \mathrm{H}), 7.19(\mathrm{~d}, J=8.0 \mathrm{~Hz}, 1 \mathrm{H}), 6.81(\mathrm{~d}, J=7.6 \mathrm{~Hz}, 1 \mathrm{H}), 2.53$ $(\mathrm{s}, 3 \mathrm{H}), 2.30(\mathrm{~s}, 3 \mathrm{H}) ;{ }^{13} \mathrm{C}$ NMR $\left(101 \mathrm{MHz}, \mathrm{DMSO}-d_{6}\right) \delta$ $(\mathrm{ppm}) 172.6\left(\mathrm{~d}, J_{\mathrm{C}-\mathrm{F}}=6.5 \mathrm{~Hz}, 1 \mathrm{C}\right), 157.8\left(\mathrm{~d}, J_{\mathrm{C}-\mathrm{F}}=34.0 \mathrm{~Hz}\right.$, 1C), 156.6, 140.5, 133.3, 131.5, 131.3, 127.0, 124.7, 115.7 (dd, $J_{\mathrm{C}-\mathrm{F}}=255.2,249.2 \mathrm{~Hz}$, 1C), 110.1, $77.2\left(\mathrm{dd}, J_{\mathrm{C}-\mathrm{F}}=31.8,24.4 \mathrm{~Hz}, 1 \mathrm{C}\right), 20.5,10.6 .{ }^{19} \mathrm{~F}$ NMR $(376 \mathrm{MHz}$, DMSO- $\left.d_{6}\right) \delta(\mathrm{ppm})-106.8(\mathrm{~d}, J=275.6 \mathrm{~Hz}, 1 \mathrm{~F}),-108.6(\mathrm{~d}, J=275.6 \mathrm{~Hz}, 1 \mathrm{~F})$. HRMS (ESI) m/z: [M - H]- Calcd for $\mathrm{C}_{14} \mathrm{H}_{10} \mathrm{~F}_{2} \mathrm{~N}_{3} \mathrm{O}_{5}$ 338.0594; Found 338.0593.

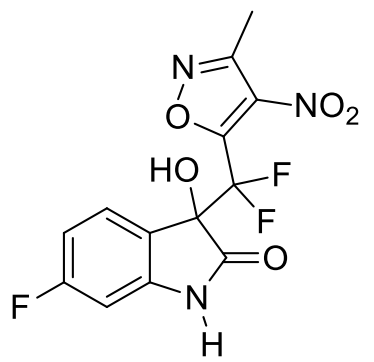

\section{3-(Difluoro(3-methyl-4-nitroisoxazol-5-yl)methyl)-6-fluoro} -3-hydroxyindolin-2-one (3j): white solid; mp: $185-187{ }^{\circ} \mathrm{C}$; $43.2 \mathrm{mg}, 84 \%$ yield, ${ }^{1} \mathrm{H}$ NMR (400 MHz, DMSO- $\left.d_{6}\right) \delta(\mathrm{ppm})$ $11.02(\mathrm{~s}, 1 \mathrm{H}), 7.68(\mathrm{~s}, 1 \mathrm{H}), 7.45(\mathrm{t}, J=6.8 \mathrm{~Hz}, 1 \mathrm{H}), 6.92-6.87$ $(\mathrm{m}, 1 \mathrm{H}), 6.77(\mathrm{~d}, J=8.8 \mathrm{~Hz}, 1 \mathrm{H}), 2.53(\mathrm{~s}, 3 \mathrm{H}) ;{ }^{13} \mathrm{C} \mathrm{NMR}(101$ $\left.\mathrm{MHz}, \mathrm{DMSO}-d_{6}\right) \delta(\mathrm{ppm}) 172.8\left(\mathrm{~d}, J_{\mathrm{C}-\mathrm{F}}=6.2 \mathrm{~Hz}, 1 \mathrm{C}\right), 163.9$ $\left(\mathrm{d}, J_{\mathrm{C}-\mathrm{F}}=244.7 \mathrm{~Hz}, 1 \mathrm{C}\right), 157.6\left(\mathrm{t}, J_{\mathrm{C}-\mathrm{F}}=33.8 \mathrm{~Hz}, 1 \mathrm{C}\right), 156.7$, $144.9\left(\mathrm{~d}, J_{\mathrm{C}-\mathrm{F}}=12.5 \mathrm{~Hz}, 1 \mathrm{C}\right), 133.3,128.3\left(\mathrm{~d}, J_{\mathrm{C}-\mathrm{F}}=10.4 \mathrm{~Hz}, 1 \mathrm{C}\right), 120.5\left(\mathrm{~d}, J_{\mathrm{C}-\mathrm{F}}=2.8\right.$ $\mathrm{Hz}, 1 \mathrm{C}), 115.5$ (dd, $\left.J_{\mathrm{C}-\mathrm{F}}=255.0,249.6 \mathrm{~Hz} 1 \mathrm{C}\right), 108.7$ (d, $\left.J_{\mathrm{C}-\mathrm{F}}=22.5 \mathrm{~Hz}, 1 \mathrm{C}\right), 98.8$ (d, $\left.J_{\mathrm{C}-\mathrm{F}}=27.1 \mathrm{~Hz}, 1 \mathrm{C}\right), 76.8\left(\mathrm{dd}, J_{\mathrm{C}-\mathrm{F}}=31.7,24.8 \mathrm{~Hz}, 1 \mathrm{C}\right), 10.7 .{ }^{19} \mathrm{~F}$ NMR $(376 \mathrm{MHz}$, DMSO-d $\left.{ }_{6}\right) \delta(\mathrm{ppm})-107.7(\mathrm{~d}, J=278.2 \mathrm{~Hz}, 1 \mathrm{~F}),-108.1(\mathrm{~s}, 1 \mathrm{~F})-108.5(\mathrm{~d}, J=278.2$ $\mathrm{Hz}, 1 \mathrm{~F}$ ). HRMS (ESI) m/z: [M - H]- Calcd for $\mathrm{C}_{13} \mathrm{H}_{7} \mathrm{~F}_{3} \mathrm{~N}_{3} \mathrm{O}_{5}$ 342.0343; Found 342.0343 . 
<smiles>Cc1noc(C(F)(F)C2(O)C(=O)Nc3cc(Cl)ccc32)c1[N+](=O)[O-]</smiles>

6-Chloro-3-(difluoro(3-methyl-4-nitroisoxazol-5-yl)methyl )-3-hydroxyindolin-2-one (3k): yellow solid; $\mathrm{mp}$ : decomposed at $178{ }^{\circ} \mathrm{C} ; 52.2 \mathrm{mg}, 97 \%$ yield, ${ }^{1} \mathrm{H}$ NMR (400 $\left.\mathrm{MHz}, \mathrm{DMSO}-d_{6}\right) \delta(\mathrm{ppm}) 11.01(\mathrm{~s}, 1 \mathrm{H}), 7.72(\mathrm{~d}, J=1.6 \mathrm{~Hz}$, $1 \mathrm{H}), 7.43(\mathrm{~d}, J=7.6 \mathrm{~Hz}, 1 \mathrm{H}), 7.14(\mathrm{dd}, J=8.21 .6 \mathrm{~Hz}, 1 \mathrm{H})$, $6.96(\mathrm{~d}, J=2.0 \mathrm{~Hz}, 1 \mathrm{H}), 2.53(\mathrm{~s}, 3 \mathrm{H}) ;{ }^{13} \mathrm{C} \mathrm{NMR}(101 \mathrm{MHz}$, DMSO- $\left.d_{6}\right) \delta(\mathrm{ppm}) 172.6\left(\mathrm{~d}, J_{\mathrm{C}-\mathrm{F}}=6.2 \mathrm{~Hz}, 1 \mathrm{C}\right), 157.5\left(\mathrm{t}, J_{\mathrm{C}-\mathrm{F}}\right.$ $=33.8 \mathrm{~Hz}, 1 \mathrm{C}), 156.8,144.5,135.8,113.4,128.0,123.4,122.2,115.5\left(\mathrm{dd}, J_{\mathrm{C}-\mathrm{F}}=\right.$ $255.5,249.8 \mathrm{~Hz}, 1 \mathrm{C}), 111.6,76.9\left(\mathrm{dd}, J_{\mathrm{C}-\mathrm{F}}=31.6,24.7 \mathrm{~Hz}, 1 \mathrm{C}\right), 10.7 .{ }^{19} \mathrm{~F}$ NMR $(376$ MHz, DMSO- $\left.d_{6}\right) \delta(\mathrm{ppm})-106.8(\mathrm{~d}, J=276.7 \mathrm{~Hz}, 1 \mathrm{~F}),-108.5(\mathrm{~d}, J=276.4 \mathrm{~Hz}, 1 \mathrm{~F})$. HRMS (ESI) m/z: [M - H]- Calcd for $\mathrm{C}_{13} \mathrm{H}_{7} \mathrm{ClF}_{2} \mathrm{~N}_{3} \mathrm{O}_{5}$ 358.0048; Found 358.0049.

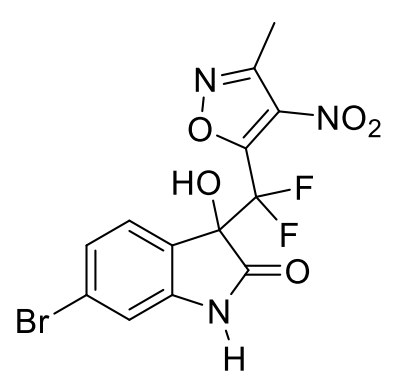

6-Bromo-3-(difluoro(3-methyl-4-nitroisoxazol-5-yl)methyl) -3-hydroxyindolin-2-one (3I): white solid; mp: 205-207 ${ }^{\circ} \mathrm{C}$; $55.8 \mathrm{mg}, 92 \%$ yield, ${ }^{1} \mathrm{H}$ NMR (400 MHz, DMSO- $\left.d_{6}\right) \delta(\mathrm{ppm})$ $11.02(\mathrm{~s}, 1 \mathrm{H}), 7.74(\mathrm{~s}, 1 \mathrm{H}), 7.38-7.28(\mathrm{~m}, 2 \mathrm{H}), 7.09(\mathrm{~s}, 1 \mathrm{H})$, $2.53(\mathrm{~s}, 3 \mathrm{H}) ;{ }^{13} \mathrm{C}$ NMR $\left(101 \mathrm{MHz}, \mathrm{DMSO}-d_{6}\right) \delta(\mathrm{ppm}) 172.5$ $\left(\mathrm{d}, J_{\mathrm{C}-\mathrm{F}}=6.4 \mathrm{~Hz}, 1 \mathrm{C}\right), 157.5\left(\mathrm{t}, J_{\mathrm{C}-\mathrm{F}}=33.8 \mathrm{~Hz}, 1 \mathrm{C}\right), 156.8$, $144.6,133.4,128.3,125.2,124.3,124.0,115.5\left(\mathrm{dd}, J_{\mathrm{C}-\mathrm{F}}=\right.$ 255.8, 249.7 Hz, 1C), 113.4, $77.0\left(\mathrm{dd}, J_{\mathrm{C}-\mathrm{F}}=31.8,24.7 \mathrm{~Hz}\right.$, 1C), 10.8. ${ }^{19} \mathrm{~F}$ NMR (376 MHz, DMSO- $\left.d_{6}\right) \delta(\mathrm{ppm})-106.8(\mathrm{~d}, J=276.7 \mathrm{~Hz}, 1 \mathrm{~F})$, $-108.5(\mathrm{~d}, J=276.7 \mathrm{~Hz}, 1 \mathrm{~F})$. HRMS (ESI) m/z: [M - H]- Calcd for $\mathrm{C}_{13} \mathrm{H}_{7} \mathrm{BrF}_{2} \mathrm{~N}_{3} \mathrm{O}_{5}$ 401.9543; Found 401.9544.

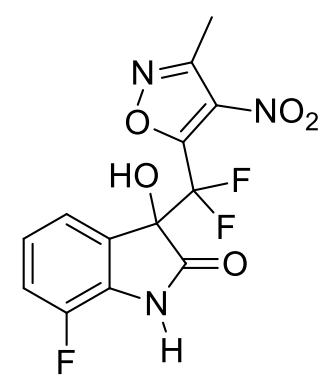

3-(Difluoro(3-methyl-4-nitroisoxazol-5-yl)methyl)-7-fluoro-3-

hydroxyindolin-2-one (3m): pale yellow solid; mp: 173-174 ${ }^{\circ} \mathrm{C}$; $50.4 \mathrm{mg}, 98 \%$ yield, ${ }^{1} \mathrm{H}$ NMR (400 MHz, DMSO- $\left.d_{6}\right) \delta(\mathrm{ppm})$ $11.44(\mathrm{~s}, 1 \mathrm{H}), 7.79(\mathrm{~s}, 1 \mathrm{H}), 7.37-7.28(\mathrm{~m}, 2 \mathrm{H}), 7.15-7.10(\mathrm{~m}, 1 \mathrm{H})$, $2.54(\mathrm{~s}, 3 \mathrm{H}) ;{ }^{13} \mathrm{C}$ NMR $\left(101 \mathrm{MHz}, \mathrm{DMSO}-d_{6}\right) \delta(\mathrm{ppm}) 172.5(\mathrm{~d}$, $\left.J_{\mathrm{C}-\mathrm{F}}=6.3 \mathrm{~Hz}, 1 \mathrm{C}\right), 157.5\left(\mathrm{t}, J_{\mathrm{C}-\mathrm{F}}=33.7 \mathrm{~Hz}, 1 \mathrm{C}\right), 156.8,146.5(\mathrm{~d}$, $\left.J_{\mathrm{C}-\mathrm{F}}=242.2 \mathrm{~Hz}, 1 \mathrm{C}\right), 133.4,130.1\left(\mathrm{t}, J_{\mathrm{C}-\mathrm{F}}=12.8 \mathrm{~Hz}, 1 \mathrm{C}\right), 127.4$ $\left(\mathrm{d}, J_{\mathrm{C}-\mathrm{F}}=3.4 \mathrm{~Hz}, 1 \mathrm{C}\right), 123.5\left(\mathrm{~d}, J_{\mathrm{C}-\mathrm{F}}=5.7 \mathrm{~Hz}, 1 \mathrm{C}\right), 122.6,118.5\left(\mathrm{~d}, J_{\mathrm{C}-\mathrm{F}}=16.9 \mathrm{~Hz}\right.$, 1C), $115.5\left(\mathrm{dd}, J_{\mathrm{C}-\mathrm{F}}=255.7,249.7 \mathrm{~Hz}, 1 \mathrm{C}\right), 77.3\left(\mathrm{dd}, J_{\mathrm{C}-\mathrm{F}}=31.6,24.5 \mathrm{~Hz}, 1 \mathrm{C}\right), 10.7$. ${ }^{19} \mathrm{~F}$ NMR $\left(376 \mathrm{MHz}, \mathrm{DMSO}-d_{6}\right) \delta(\mathrm{ppm})-106.8(\mathrm{~d}, J=276.7 \mathrm{~Hz}, 1 \mathrm{~F}),-108.6(\mathrm{~d}, J=$ $277.1 \mathrm{~Hz}, 1 \mathrm{~F}$ ), 132.3 (s, 1F). HRMS (ESI) m/z: [M - H]- Calcd for $\mathrm{C}_{13} \mathrm{H}_{7} \mathrm{~F}_{3} \mathrm{~N}_{3} \mathrm{O}_{5}$ 342.0343; Found 342.0342.<smiles>Cc1noc(C(F)(F)C2(O)C(=O)Nc3c(Br)cccc32)c1[N+](=O)[O-]</smiles>

7-Bromo-3-(difluoro(3-methyl-4-nitroisoxazol-5-yl)methyl)-3hydroxyindolin-2-one (3n): pale yellow solid; mp: $188-190{ }^{\circ} \mathrm{C}$; $57.6 \mathrm{mg}, 95 \%$ yield, ${ }^{1} \mathrm{H}$ NMR (400 MHz, DMSO- $\left.d_{6}\right) \delta(\mathrm{ppm})$ $11.19(\mathrm{~s}, 1 \mathrm{H}), 7.78(\mathrm{~d}, J=1.2 \mathrm{~Hz}, 1 \mathrm{H}), 7.60(\mathrm{~d}, J=8.0 \mathrm{~Hz}, 1 \mathrm{H})$, $7.44(\mathrm{~d}, J=7.2 \mathrm{~Hz}, 1 \mathrm{H}), 7.06$ (t, $J=7.8 \mathrm{~Hz}, 1 \mathrm{H}), 2.54$ (s, 3H); ${ }^{13} \mathrm{C}$ NMR (101 MHz, DMSO- $\left.d_{6}\right) \delta(\mathrm{ppm}) 172.6\left(\mathrm{~d}, J_{\mathrm{C}-\mathrm{F}}=6.3 \mathrm{~Hz}\right.$, $1 \mathrm{C}), 157.4\left(\mathrm{t}, J_{\mathrm{C}-\mathrm{F}}=33.7 \mathrm{~Hz}, 1 \mathrm{C}\right), 156.8,142.4,134.2,133.4$, 
126.4, 125.6, 124.2, $115.5\left(\mathrm{dd}, J_{\mathrm{C}-\mathrm{F}}=255.8,249.6 \mathrm{~Hz}, 1 \mathrm{C}\right), 102.7,77.9\left(\mathrm{dd}, J_{\mathrm{C}-\mathrm{F}}=\right.$ 31.8, $24.7 \mathrm{~Hz}, 1 \mathrm{C}), 10.8 .{ }^{19} \mathrm{~F}$ NMR $\left(376 \mathrm{MHz}, \mathrm{DMSO}-d_{6}\right) \delta(\mathrm{ppm})-107.1(\mathrm{~d}, J=$ $277.1 \mathrm{~Hz}, 1 \mathrm{~F}),-108.5$ (d, $J=277.1 \mathrm{~Hz}, 1 \mathrm{~F}$ ). HRMS (ESI) m/z: [M - H]- Calcd for $\mathrm{C}_{13} \mathrm{H}_{7} \mathrm{BrF}_{2} \mathrm{~N}_{3} \mathrm{O}_{5} 401.9543$; Found 401.9541.

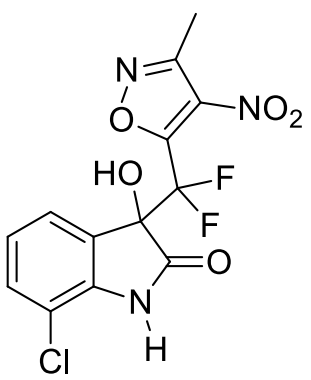

7-Chloro-3-(difluoro(3-methyl-4-nitroisoxazol-5-yl)methyl)-3-

hydroxyindolin-2-one (3o): yellow solid; mp: 192-193 ${ }^{\circ} \mathrm{C} ; 53.4$ mg, 99\% yield, ${ }^{1} \mathrm{H}$ NMR (400 MHz, DMSO- $\left.d_{6}\right) \delta(\mathrm{ppm}) 11.35$ (s, 1H), $7.79(\mathrm{~s}, 1 \mathrm{H}), 7.45(\mathrm{~d}, J=29.6 \mathrm{~Hz}, 2 \mathrm{H}), 7.13(\mathrm{~s}, 1 \mathrm{H}), 2.54(\mathrm{~s}$, $3 \mathrm{H}) ;{ }^{13} \mathrm{C}$ NMR $\left(101 \mathrm{MHz}, \mathrm{DMSO}-d_{6}\right) \delta(\mathrm{ppm}) 172.7\left(\mathrm{~d}, J_{\mathrm{C}-\mathrm{F}}=\right.$ $5.9 \mathrm{~Hz}, 1 \mathrm{C}), 157.4$ (t, $\left.J_{\mathrm{C}-\mathrm{F}}=33.3 \mathrm{~Hz}, 1 \mathrm{C}\right), 156.8,140.7,133.4$, $131.3,126.4,125.1,123.8,115.5\left(\mathrm{dd}, J_{\mathrm{C}-\mathrm{F}}=256.0,248.9 \mathrm{~Hz}, 1 \mathrm{C}\right)$, $114.7,77.7\left(\mathrm{dd}, J_{\mathrm{C}-\mathrm{F}}=32.0,25.0 \mathrm{~Hz}, 1 \mathrm{C}\right), 10.8 .{ }^{19} \mathrm{~F}$ NMR $\left(376 \mathrm{MHz}, \mathrm{DMSO}-d_{6}\right) \delta$ (ppm) $-106.8(\mathrm{~d}, J=277.1 \mathrm{~Hz}, 1 \mathrm{~F}),-108.6(\mathrm{~d}, J=277.1 \mathrm{~Hz}, 1 \mathrm{~F}) . \mathrm{HRMS}(\mathrm{ESI}) \mathrm{m} / \mathrm{z}$ : [M - H]- Calcd for $\mathrm{C}_{13} \mathrm{H}_{7} \mathrm{ClF}_{2} \mathrm{~N}_{3} \mathrm{O}_{5}$ 358.0048; Found 358.0048.

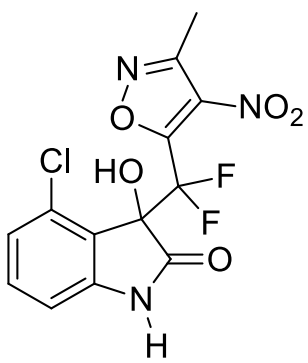

4-Chloro-3-(difluoro(3-methyl-4-nitroisoxazol-5-yl)methyl)

-3-hydroxyindolin-2-one (3p): yellow solid; mp: 210-213 ${ }^{\circ} \mathrm{C}$; $53.4 \mathrm{mg}, 99 \%$ yield, ${ }^{1} \mathrm{H}$ NMR (400 MHz, DMSO- $\left.d_{6}\right) \delta(\mathrm{ppm})$ $11.05(\mathrm{~s}, 1 \mathrm{H}), 7.57(\mathrm{~s}, 1 \mathrm{H}), 7.38(\mathrm{t}, J=7.8 \mathrm{~Hz}, 1 \mathrm{H}), 7.08(\mathrm{~d}, J$ $=8.0 \mathrm{~Hz}, 1 \mathrm{H}), 6.89(\mathrm{~d}, J=7.6 \mathrm{~Hz}, 1 \mathrm{H}), 2.53(\mathrm{~s}, 3 \mathrm{H}) ;{ }^{13} \mathrm{C}$ NMR $\left(101 \mathrm{MHz}, \mathrm{DMSO}-d_{6}\right) \delta(\mathrm{ppm}) 172.7\left(\mathrm{~d}, J_{\mathrm{C}-\mathrm{F}}=6.4 \mathrm{~Hz}\right.$, $1 \mathrm{C}), 157.7\left(\mathrm{t}, J_{\mathrm{C}-\mathrm{F}}=33.6 \mathrm{~Hz}, 1 \mathrm{C}\right), 156.7,145.2,133.4,132.8$, $132.1,124.0,121.4,116.0\left(\mathrm{dd}, J_{\mathrm{C}-\mathrm{F}}=260.2,249.5 \mathrm{~Hz}, 1 \mathrm{C}\right), 109.4,79.2\left(\mathrm{dd}, J_{\mathrm{C}-\mathrm{F}}=\right.$ 31.7, 25.5 Hz, 1C), 10.7. ${ }^{19} \mathrm{~F}$ NMR (376 MHz, DMSO- $\left.d_{6}\right) \delta(\mathrm{ppm})-101.1(\mathrm{~d}, J=$ $273.0 \mathrm{~Hz}, 1 \mathrm{~F}),-108.4(\mathrm{~d}, J=273.0 \mathrm{~Hz}, 1 \mathrm{~F})$. HRMS (ESI) m/z: [M - H]- Calcd for $\mathrm{C}_{13} \mathrm{H}_{7} \mathrm{ClF}_{2} \mathrm{~N}_{3} \mathrm{O}_{5}$ 358.0048; Found 358.0047.

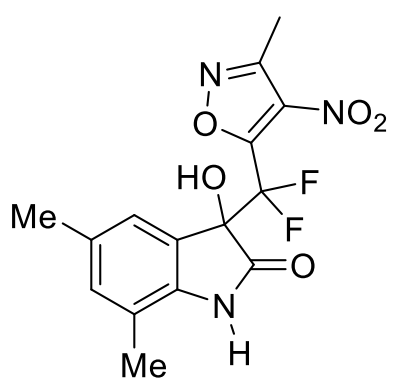

\section{3-(Difluoro(3-methyl-4-nitroisoxazol-5-yl)methyl)-3-hydr} oxy-5,7-dimethylindolin-2-one (3q): yellow oil; $44.5 \mathrm{mg}$, $84 \%$ yield, ${ }^{1} \mathrm{H}$ NMR (400 MHz, DMSO- $\left.d_{6}\right) \delta(\mathrm{ppm}) 10.78(\mathrm{~s}$, $1 \mathrm{H}), 7.50(\mathrm{~s}, 1 \mathrm{H}), 7.08(\mathrm{~s}, 1 \mathrm{H}), 7.01(\mathrm{~s}, 1 \mathrm{H}), 2.53(\mathrm{~s}, 3 \mathrm{H})$, $2.26(\mathrm{~s}, 3 \mathrm{H}), 2.18(\mathrm{~s}, 3 \mathrm{H}) ;{ }^{13} \mathrm{C}$ NMR (101 MHz, DMSO- $\left.d_{6}\right)$ $\delta(\mathrm{ppm}) 173.2\left(\mathrm{~d}, J_{\mathrm{C}-\mathrm{F}}=6.4 \mathrm{~Hz}, 1 \mathrm{C}\right), 157.9\left(\mathrm{t}, J_{\mathrm{C}-\mathrm{F}}=33.8 \mathrm{~Hz}\right.$, 1C), 156.7, 139.1, 133.4, 133.0, 131.3, 124.4, 124.3, 120.0, $115.8\left(\mathrm{dd}, J_{\mathrm{C}-\mathrm{F}}=255.1,249.0 \mathrm{~Hz}, 1 \mathrm{C}\right), 77.4\left(\mathrm{dd}, J_{\mathrm{C}-\mathrm{F}}=31.7\right.$, $24.5 \mathrm{~Hz}, 1 \mathrm{C}), 20.5,16.2,10.8 .{ }^{19} \mathrm{~F}$ NMR $\left(376 \mathrm{MHz}, \mathrm{DMSO}-d_{6}\right) \delta(\mathrm{ppm})-106.7$ (d, $J=$ $276.0 \mathrm{~Hz}, 1 \mathrm{~F}),-108.5$ (d, $J=275.6 \mathrm{~Hz}, 1 \mathrm{~F})$. HRMS (ESI) m/z: [M - H]- Calcd for $\mathrm{C}_{15} \mathrm{H}_{12} \mathrm{~F}_{2} \mathrm{~N}_{3} \mathrm{O}_{5} 352.0751$; Found 352.0748. 


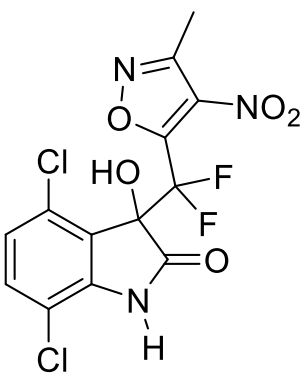

4,7-Dichloro-3-(difluoro(3-methyl-4-nitroisoxazol-5-yl)methyl

)-3-hydroxyindolin-2-one (3r): light yellow solid; mp: 169-171 ${ }^{\circ} \mathrm{C}$; $49.6 \mathrm{mg}, 84 \%$ yield, ${ }^{1} \mathrm{H}$ NMR (400 MHz, DMSO- $\left.d_{6}\right) \delta(\mathrm{ppm})$ $11.55(\mathrm{~s}, 1 \mathrm{H}), 7.74(\mathrm{~d}, J=2.8 \mathrm{~Hz}, 1 \mathrm{H}), 7.50(\mathrm{~d}, J=8.8 \mathrm{~Hz}, 1 \mathrm{H})$, $7.13(\mathrm{~d}, J=8.4 \mathrm{~Hz}, 1 \mathrm{H}), 2.54(\mathrm{~s}, 3 \mathrm{H}) ;{ }^{13} \mathrm{C} \mathrm{NMR}(101 \mathrm{MHz}$, DMSO- $\left.d_{6}\right) \delta(\mathrm{ppm}) 172.8\left(\mathrm{~d}, J_{\mathrm{C}-\mathrm{F}}=6.5 \mathrm{~Hz}, 1 \mathrm{C}\right), 157.4\left(\mathrm{t}, J_{\mathrm{C}-\mathrm{F}}=\right.$ $33.4 \mathrm{~Hz}, 1 \mathrm{C}), 156.8,142.7,133.5,132.6,130.9,125.2,122.9$, $115.9\left(\mathrm{dd}, J_{\mathrm{C}-\mathrm{F}}=260.7,249.3 \mathrm{~Hz}, 1 \mathrm{C}\right), 113.8,79.9\left(\mathrm{dd}, J_{\mathrm{C}-\mathrm{F}}=31.9,25.4 \mathrm{~Hz}, 1 \mathrm{C}\right), 10.7$ ${ }^{19} \mathrm{~F}$ NMR (376 MHz, DMSO- $\left.d_{6}\right) \delta(\mathrm{ppm})-101.0(\mathrm{~d}, J=273.7 \mathrm{~Hz}, 1 \mathrm{~F}),-108.4(\mathrm{~d}, J=$ $273.7 \mathrm{~Hz}, 1 \mathrm{~F}$ ). HRMS (ESI) m/z: [M - H]- Calcd for $\mathrm{C}_{13} \mathrm{H}_{6} \mathrm{Cl}_{2} \mathrm{~F}_{2} \mathrm{~N}_{3} \mathrm{O}_{5} 391.9658$; Found 391.9660 .

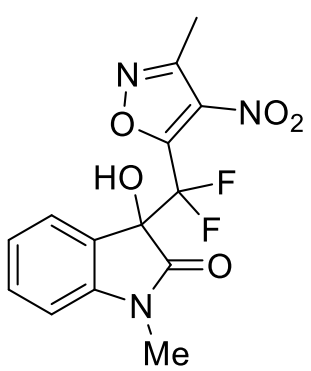

\section{3-(Difluoro(3-methyl-4-nitroisoxazol-5-yl)methyl)-3-hydroxy-}

1-methylindolin-2-one (3s): white solid; mp: 128-139 ${ }^{\circ} \mathrm{C} ; 50.3$ mg, 99\% yield, ${ }^{1} \mathrm{H}$ NMR (400 MHz, DMSO- $\left.d_{6}\right) \delta(\mathrm{ppm}) 7.69(\mathrm{~s}$, $1 \mathrm{H}), 7.47(\mathrm{~s}, 2 \mathrm{H}), 7.15(\mathrm{~s}, 2 \mathrm{H}), 3.12(\mathrm{~s}, 3 \mathrm{H}), 2.53(\mathrm{~s}, 3 \mathrm{H}) ;{ }^{13} \mathrm{C}$ NMR (101 MHz, DMSO- $\left.d_{6}\right) \delta(\mathrm{ppm}) 170.9\left(\mathrm{~d}, J_{\mathrm{C}-\mathrm{F}}=6.4 \mathrm{~Hz}, 1 \mathrm{C}\right)$, $157.6\left(\mathrm{t}, J_{\mathrm{C}-\mathrm{F}}=33.8 \mathrm{~Hz}, 1 \mathrm{C}\right), 156.8,144.3,133.3,131.5,126.1$, $124.0,123.1,115.6\left(\mathrm{dd}, J_{\mathrm{C}-\mathrm{F}}=255.7,250.0 \mathrm{~Hz}, 1 \mathrm{C}\right), 109.5,77.0$ $\left(\mathrm{dd}, J_{\mathrm{C}-\mathrm{F}}=31.3,25.3 \mathrm{~Hz}, 1 \mathrm{C}\right), 26.3,10.8 .{ }^{19} \mathrm{~F}$ NMR $\left(376 \mathrm{MHz}, \mathrm{DMSO}-d_{6}\right) \delta(\mathrm{ppm})$ -106.9 (d, $J=275.6 \mathrm{~Hz}, 1 \mathrm{~F}),-108.4$ (d, $J=276.0 \mathrm{~Hz}, 1 \mathrm{~F})$. HRMS (ESI) m/z: [M + $\mathrm{Na}]+$ Calcd for $\mathrm{C}_{14} \mathrm{H}_{11} \mathrm{~F}_{2} \mathrm{~N}_{3} \mathrm{O}_{5} \mathrm{Na}$ 362.0546; Found 362.0552.

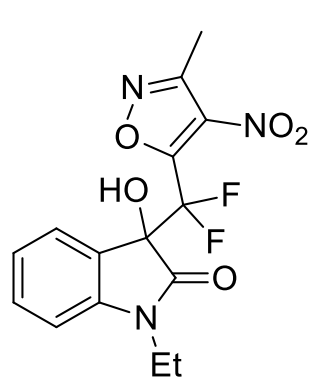

3-(Difluoro(3-methyl-4-nitroisoxazol-5-yl)methyl)-1-ethyl-3-h ydroxyindolin-2-one (3t): white solid; $\mathrm{mp}$ : $185-187^{\circ} \mathrm{C} ; 52.5 \mathrm{mg}$, 99\% yield, ${ }^{1} \mathrm{H}$ NMR (400 MHz, DMSO- $\left.d_{6}\right) \delta(\mathrm{ppm}) 7.70(\mathrm{~s}, 1 \mathrm{H})$, 7.49 (s, 2H), $7.18(\mathrm{~s}, 2 \mathrm{H}), 3.68(\mathrm{dd}, J=24.6,5.8 \mathrm{~Hz}, 2 \mathrm{H}), 2.54(\mathrm{~s}$, $3 \mathrm{H}), 1.14(\mathrm{~s}, 3 \mathrm{H}) ;{ }^{13} \mathrm{C}$ NMR $\left(101 \mathrm{MHz}, \mathrm{DMSO}-d_{6}\right) \delta(\mathrm{ppm})$ $170.6\left(\mathrm{~d}, J_{\mathrm{C}-\mathrm{F}}=6.2 \mathrm{~Hz}, 1 \mathrm{C}\right), 157.7\left(\mathrm{t}, J_{\mathrm{C}-\mathrm{F}}=33.4 \mathrm{~Hz}, 1 \mathrm{C}\right), 156.8$, $143.3,133.3,131.5,126.3,124.2,122.9,115.6\left(\mathrm{dd}, J_{\mathrm{C}-\mathrm{F}}=255.4\right.$, $250.1 \mathrm{~Hz}, 1 \mathrm{C}), 109.5,76.9\left(\mathrm{dd}, J_{\mathrm{C}-\mathrm{F}}=30.9,25.0 \mathrm{~Hz}, 1 \mathrm{C}\right), 34.5$, 12.2, 10.8. ${ }^{19} \mathrm{~F}$ NMR (376 MHz, DMSO- $\left.d_{6}\right) \delta(\mathrm{ppm})-107.0(\mathrm{~d}, J=275.6 \mathrm{~Hz}, 1 \mathrm{~F})$, $-108.3(\mathrm{~d}, J=276.0 \mathrm{~Hz}, 1 \mathrm{~F})$. HRMS (ESI) $\mathrm{m} / \mathrm{z}:[\mathrm{M}+\mathrm{Na}]+$ Calcd for $\mathrm{C}_{15} \mathrm{H}_{13} \mathrm{~F}_{2} \mathrm{~N}_{3} \mathrm{O}_{5} \mathrm{Na}$ 376.0721; Found 376.0708.

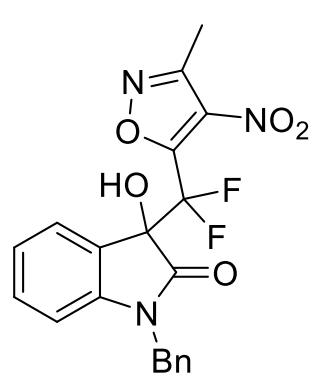

1-Benzyl-3-(difluoro(3-methyl-4-nitroisoxazol-5-yl)methyl)-

3-hydroxyindolin-2-one (3u): pale yellow solid; mp: 178-181 ${ }^{\circ} \mathrm{C} ; 57.3 \mathrm{mg}, 92 \%$ yield, ${ }^{1} \mathrm{H}$ NMR (400 MHz, DMSO- $\left.d_{6}\right) \delta$ (ppm) $7.87(\mathrm{~d}, J=2.0 \mathrm{~Hz}, 1 \mathrm{H}), 7.50(\mathrm{~d}, J=7.6 \mathrm{~Hz}, 1 \mathrm{H})$, 7.42-7.38 (m, 1H), 7.35-7.33 (m, 4H), 7.31-7.26 (m, 1H), 7.15 $(\mathrm{t}, J=7.4 \mathrm{~Hz} 1 \mathrm{H}), 6.99(\mathrm{~d}, J=7.6 \mathrm{~Hz} 1 \mathrm{H}), 4.97(\mathrm{~d}, J=3.9 \mathrm{~Hz}$, $1 \mathrm{H}), 4.82(\mathrm{~d}, J=3.9 \mathrm{~Hz}, 1 \mathrm{H}), 2.55(\mathrm{~s}, 3 \mathrm{H}) ;{ }^{13} \mathrm{C} \mathrm{NMR}(101$ 
$\left.\mathrm{MHz}, \mathrm{DMSO}-d_{6}\right) \delta(\mathrm{ppm}) 171.2\left(\mathrm{~d}, J_{\mathrm{C}-\mathrm{F}}=6.7 \mathrm{~Hz}, 1 \mathrm{C}\right), 157.6\left(\mathrm{t}, J_{\mathrm{C}-\mathrm{F}}=33.8 \mathrm{~Hz}, 1 \mathrm{C}\right)$, $156.8,143.3,135.5,133.4,131.4,128.7,127.6,127.3,126.4,124.0,123.2,115.7(\mathrm{dd}$, $\left.J_{\mathrm{C}-\mathrm{F}}=255.8,249.7 \mathrm{~Hz}, 1 \mathrm{C}\right), 111.0,77.1\left(\mathrm{dd}, J_{\mathrm{C}-\mathrm{F}}=31.7,25.0 \mathrm{~Hz}, 1 \mathrm{C}\right), 42.9,10.8 .{ }^{19} \mathrm{~F}$ NMR (376 MHz, DMSO-d $d_{6} \delta(\mathrm{ppm})-106.6(\mathrm{~d}, J=276.0 \mathrm{~Hz}, 1 \mathrm{~F}),-108.4(\mathrm{~d}, J=$ $276.4 \mathrm{~Hz}, 1 \mathrm{~F}$ ). HRMS (ESI) $\mathrm{m} / \mathrm{z}$ : [M $+\mathrm{Na}]+$ Calcd for $\mathrm{C}_{20} \mathrm{H}_{15} \mathrm{~F}_{2} \mathrm{~N}_{3} \mathrm{O}_{5} \mathrm{Na} 438.0887$; Found 438.0864 .

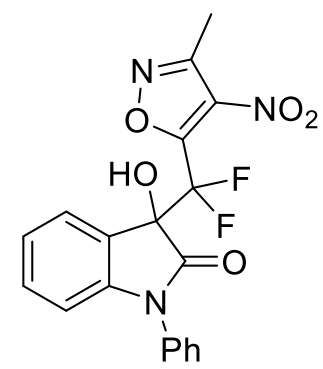

3-(Difluoro(3-methyl-4-nitroisoxazol-5-yl)methyl)-3-hydroxy -1-phenylindolin-2-one (3v): ivory white solid; $\mathrm{mp}$ : decomposed at $225{ }^{\circ} \mathrm{C} ; 51.1 \mathrm{mg}, 85 \%$ yield, ${ }^{1} \mathrm{H}$ NMR $(400 \mathrm{MHz}$, DMSO- $\left.d_{6}\right) \delta(\mathrm{ppm}) 7.93(\mathrm{~s}, 1 \mathrm{H}), 7.63-7.60(\mathrm{~m}, 3 \mathrm{H}), 7.51(\mathrm{t}, J=$ $7.2 \mathrm{~Hz}, 1 \mathrm{H}), 7.45(\mathrm{~d}, J=7.2 \mathrm{~Hz}, 1 \mathrm{H}), 7.38(\mathrm{~d}, J=7.6 \mathrm{~Hz}, 2 \mathrm{H})$, $7.24(\mathrm{t}, J=7.2 \mathrm{~Hz}, 1 \mathrm{H}), 6.85(\mathrm{~d} J=8.0 \mathrm{~Hz}, 1 \mathrm{H}), 2.54(\mathrm{~s}, 3 \mathrm{H})$; ${ }^{13} \mathrm{C}$ NMR $\left(101 \mathrm{MHz}, \mathrm{DMSO}-d_{6}\right) \delta(\mathrm{ppm}) 170.6\left(\mathrm{~d}, J_{\mathrm{C}-\mathrm{F}}=6.0 \mathrm{~Hz}\right.$, 1C), $157.7\left(\mathrm{t}, J_{\mathrm{C}-\mathrm{F}}=33.8 \mathrm{~Hz}, 1 \mathrm{C}\right), 156.9,144.1,133.3,133.2,131.6,130.0,128.7$, 126.7, 126.4, 123.9. 123.8, $115.6\left(\mathrm{dd}, J_{\mathrm{C}-\mathrm{F}}=255.7,250.2 \mathrm{~Hz}, 1 \mathrm{C}\right), 109.8,77.1(\mathrm{dd}$, $\left.J_{\mathrm{C}-\mathrm{F}}=30.8,25.3 \mathrm{~Hz}, 1 \mathrm{C}\right), 10.8 .{ }^{19} \mathrm{~F} \mathrm{NMR}\left(376 \mathrm{MHz}, \mathrm{DMSO}-d_{6}\right) \delta(\mathrm{ppm})-106.8(\mathrm{~d}, J$ $=276.7 \mathrm{~Hz}, 1 \mathrm{~F}),-108.0(\mathrm{~d}, J=277.7 \mathrm{~Hz}, 1 \mathrm{~F})$. HRMS (ESI) m/z: $[\mathrm{M}+\mathrm{Na}]+$ Calcd for $\mathrm{C}_{19} \mathrm{H}_{13} \mathrm{~F}_{2} \mathrm{~N}_{3} \mathrm{O}_{5} \mathrm{Na} 424.0721$; Found 424.0707.

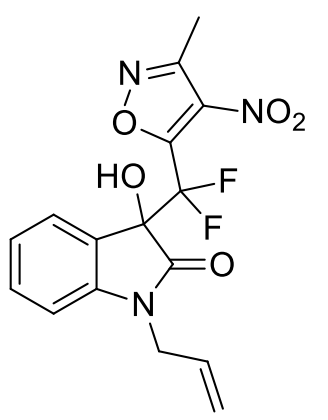

1-Allyl-3-(difluoro(3-methyl-4-nitroisoxazol-5-yl)methyl)-3-hy droxyindolin-2-one (3w): pale yellow solid; mp: 152-154 ${ }^{\circ} \mathrm{C}$; $52.0 \mathrm{mg}, 95 \%$ yield, ${ }^{1} \mathrm{H}$ NMR (400 MHz, DMSO- $\left.d_{6}\right) \delta(\mathrm{ppm})$ $7.76(\mathrm{~s}, 1 \mathrm{H}), 7.50-7.44(\mathrm{~m}, 2 \mathrm{H}), 7.17(\mathrm{t}, J=7.4 \mathrm{~Hz}, 1 \mathrm{H}), 7.06(\mathrm{~d}$, $J=8.0 \mathrm{~Hz}, 1 \mathrm{H}), 5.84-5.75(\mathrm{~m}, 1 \mathrm{H}), 5.19(\mathrm{~d}, J=12.8 \mathrm{~Hz}, 2 \mathrm{H})$, 4.38-4.20 (m, 2H), $2.53(\mathrm{~s}, 3 \mathrm{H}) ;{ }^{13} \mathrm{C}$ NMR (101 MHz, DMSO- $\left.d_{6}\right)$ $\delta(\mathrm{ppm}) 170.9\left(\mathrm{~d}, J_{\mathrm{C}-\mathrm{F}}=6.4 \mathrm{~Hz}, 1 \mathrm{C}\right), 157.7\left(\mathrm{t}, J_{\mathrm{C}-\mathrm{F}}=33.8 \mathrm{~Hz}, 1 \mathrm{C}\right)$, $156.9,143.5,133.4,131.5,131.1,126.3,124.1,123.2,117.2$,

$115.7\left(\mathrm{dd}, J_{\mathrm{C}-\mathrm{F}}=255.4,249.8 \mathrm{~Hz}, 1 \mathrm{C}\right), 111.1,77.1\left(\mathrm{dd}, J_{\mathrm{C}-\mathrm{F}}=31.4,25.1 \mathrm{~Hz}, 1 \mathrm{C}\right), 41.7$, 10.8. ${ }^{19} \mathrm{~F}$ NMR (376 MHz, DMSO- $\left.d_{6}\right) \delta(\mathrm{ppm})-106.9(\mathrm{~d}, J=278.2 \mathrm{~Hz}, 1 \mathrm{~F}),-108.5(\mathrm{~d}$, $J=274.5 \mathrm{~Hz}, 1 \mathrm{~F})$. HRMS (ESI) $\mathrm{m} / \mathrm{z}$ : $[\mathrm{M}+\mathrm{H}]+$ Calcd for $\mathrm{C}_{16} \mathrm{H}_{14} \mathrm{~F}_{2} \mathrm{~N}_{3} \mathrm{O}_{5}$ 366.0902; Found 366.0896 .

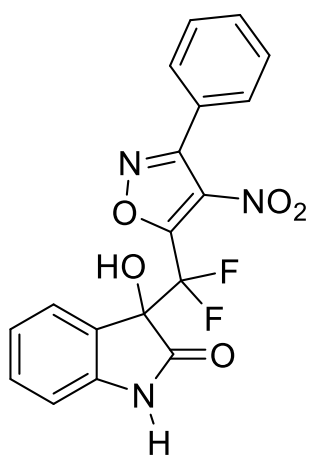

3-(Difluoro(4-nitro-3-phenylisoxazol-5-yl)methyl)-3-hydroxyi ndolin-2-one (4a): white solid; mp: $153-154{ }^{\circ} \mathrm{C} ; 52.3 \mathrm{mg}, 90 \%$ yield, ${ }^{1} \mathrm{H}$ NMR (400 MHz, DMSO- $\left.d_{6}\right) \delta(\mathrm{ppm}) 10.91(\mathrm{~s}, 1 \mathrm{H})$, $7.78(\mathrm{~s}, 1 \mathrm{H}), 7.68-7.58(\mathrm{~m}, 5 \mathrm{H}), 7.46(\mathrm{~d}, J=7.2 \mathrm{~Hz}, 1 \mathrm{H}), 7.40(\mathrm{t}$, $J=7.8 \mathrm{~Hz}, 1 \mathrm{H}), 7.11(\mathrm{t}, J=7.4 \mathrm{~Hz}, 1 \mathrm{H}), 6.95(\mathrm{~d}, J=7.6 \mathrm{~Hz}, 1 \mathrm{H})$; ${ }^{13} \mathrm{C}$ NMR (101 MHz, DMSO- $\left.d_{6}\right) \delta(\mathrm{ppm}) 172.7\left(\mathrm{~d}, J_{\mathrm{C}-\mathrm{F}}=6.0 \mathrm{~Hz}\right.$, $1 \mathrm{C}), 158.1\left(\mathrm{t}, J_{\mathrm{C}-\mathrm{F}}=34.0 \mathrm{~Hz}, 1 \mathrm{C}\right), 157.7,143.1,132.4,131.5(\mathrm{~d}$, $\left.J_{\mathrm{C}-\mathrm{F}}=2.0 \mathrm{~Hz}, 1 \mathrm{C}\right), 131.4,129.2,128.5\left(\mathrm{~d}, J_{\mathrm{C}-\mathrm{F}}=7.2 \mathrm{~Hz}, 1 \mathrm{C}\right)$, $126.6,124.6,124.5\left(\mathrm{~d}, J_{\mathrm{C}-\mathrm{F}}=6.4 \mathrm{~Hz}, 1 \mathrm{C}\right), 122.5,115.7\left(\mathrm{dd}, J_{\mathrm{C}-\mathrm{F}}=\right.$ 
255.6, 248.4 Hz, 1C), 110.6, $77.3\left(\mathrm{dd}, J_{\mathrm{C}-\mathrm{F}}=32.2,25.0 \mathrm{~Hz}, 1 \mathrm{C}\right) .{ }^{19} \mathrm{~F}$ NMR $(471 \mathrm{MHz}$, DMSO- $\left.d_{6}\right) \delta(\mathrm{ppm})-106.4(\mathrm{~d}, J=278.3 \mathrm{~Hz}, 1 \mathrm{~F}),-108.5$ (d, $\left.J=278.8 \mathrm{~Hz}, 1 \mathrm{~F}\right)$. HRMS (ESI) $\mathrm{m} / \mathrm{z}$ : $[\mathrm{M}+\mathrm{H}]+$ Calcd for $\mathrm{C}_{18} \mathrm{H}_{12} \mathrm{~F}_{2} \mathrm{~N}_{3} \mathrm{O}_{5} 388.1422$; Found 388.1449.

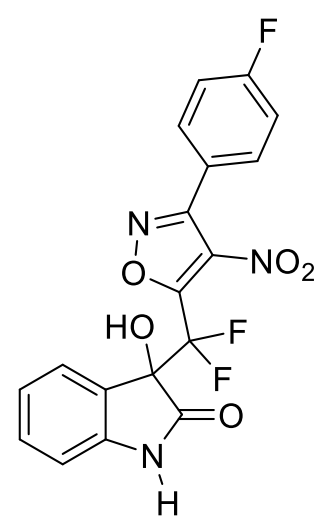

3-(Difluoro(3-(4-fluorophenyl)-4-nitroisoxazol-5-yl)methyl)-3 -hydroxyindolin-2-one (4b): white solid; mp: $181-183{ }^{\circ} \mathrm{C} ; 56.2$ mg, 92\% yield, ${ }^{1} \mathrm{H}$ NMR (400 MHz, DMSO- $\left.d_{6}\right) \delta$ (ppm) 10.90 $(\mathrm{s}, 1 \mathrm{H}), 7.76(\mathrm{~s}, 3 \mathrm{H}), 7.47-7.38(\mathrm{~m}, 4 \mathrm{H}), 7.11(\mathrm{t}, J=7.4 \mathrm{~Hz}, 1 \mathrm{H})$, $6.95(\mathrm{~d}, J=7.6 \mathrm{~Hz}, 1 \mathrm{H}) ;{ }^{13} \mathrm{C} \mathrm{NMR}\left(101 \mathrm{MHz}, \mathrm{DMSO}-d_{6}\right) \delta$ $(\mathrm{ppm}) 172.4\left(\mathrm{~d}, J_{\mathrm{C}-\mathrm{F}}=6.7 \mathrm{~Hz}, 1 \mathrm{C}\right), 163.6\left(\mathrm{~d}, J_{\mathrm{C}-\mathrm{F}}=248.1 \mathrm{~Hz}\right.$, 1C), $157.9\left(\mathrm{t}, J_{\mathrm{C}-\mathrm{F}}=34.3 \mathrm{~Hz}, 1 \mathrm{C}\right), 156.7,142.8,132.2,131.2$, $131.0\left(\mathrm{~d}, J_{\mathrm{C}-\mathrm{F}}=9.0 \mathrm{~Hz}, 1 \mathrm{C}\right), 126.3,124.2,122.2,120.9\left(\mathrm{~d}, J_{\mathrm{C}-\mathrm{F}}=\right.$ $3.1 \mathrm{~Hz}, 1 \mathrm{C}), 116.1\left(\mathrm{~d}, J_{\mathrm{C}-\mathrm{F}}=22.1 \mathrm{~Hz}, 1 \mathrm{C}\right), 115.4\left(\mathrm{dd}, J_{\mathrm{C}-\mathrm{F}}=\right.$ 255.4, 248.2 Hz, 1C), 110.3, $77.0\left(\mathrm{dd}, J_{\mathrm{C}-\mathrm{F}}=32.0,24.2 \mathrm{~Hz}, 1 \mathrm{C}\right)$.

${ }^{19} \mathrm{~F}$ NMR (471 MHz, DMSO- $\left.d_{6}\right) \delta(\mathrm{ppm})-106.5(\mathrm{~d}, J=278.3 \mathrm{~Hz}, 1 \mathrm{~F}),-108.7(\mathrm{~s}, 1 \mathrm{~F})$, $-109.5(\mathrm{~d}, J=278.3 \mathrm{~Hz}, 1 \mathrm{~F})$. HRMS (ESI) $\mathrm{m} / \mathrm{z}:[\mathrm{M}+\mathrm{Na}]+$ Calcd for $\mathrm{C}_{18} \mathrm{H}_{10} \mathrm{~F}_{3} \mathrm{~N}_{3} \mathrm{O}_{5} \mathrm{Na}$ 428.0461; Found 428.0458.

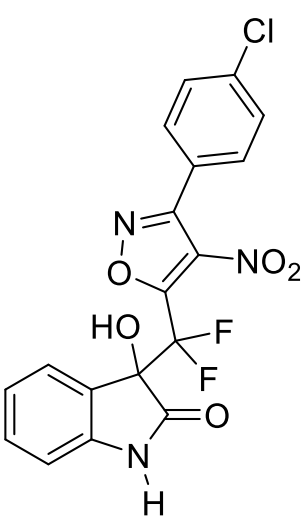

\section{3-((3-(4-Chlorophenyl)-4-nitroisoxazol-5-yl)difluoromethyl)-3}

-hydroxyindolin-2-one (4c): yellow solid; mp: 187-188 ${ }^{\circ} \mathrm{C} ; 59.6$ mg, 94\% yield, ${ }^{1} \mathrm{H}$ NMR (400 MHz, DMSO- $\left.d_{6}\right) \delta(\mathrm{ppm}) 10.90(\mathrm{~s}$, $1 \mathrm{H}), 7.75-7.66(\mathrm{~m}, 5 \mathrm{H}), 7.46(\mathrm{~d}, J=7.2 \mathrm{~Hz}, 1 \mathrm{H}), 7.40(\mathrm{t}, J=7.6$ $\mathrm{Hz}, 1 \mathrm{H}), 7.10(\mathrm{t}, J=7.6 \mathrm{~Hz}, 1 \mathrm{H}), 6.94(\mathrm{~d}, J=7.6 \mathrm{~Hz}, 1 \mathrm{H}) ;{ }^{13} \mathrm{C}$ NMR (101 MHz, DMSO- $\left.d_{6}\right) \delta(\mathrm{ppm}) 172.6\left(\mathrm{~d}, J_{\mathrm{C}-\mathrm{F}}=5.5 \mathrm{~Hz}, 1 \mathrm{C}\right)$, $158.3\left(\mathrm{t}, J_{\mathrm{C}-\mathrm{F}}=34.1 \mathrm{~Hz}, 1 \mathrm{C}\right), 156.9,143.0,136.4,132.4,131.4$, $130.5,129.2,126.5,124.4,123.5,122.4,115.6\left(\mathrm{dd}, J_{\mathrm{C}-\mathrm{F}}=255.9\right.$, $248.7 \mathrm{~Hz}, 1 \mathrm{C}), 110.5,77.2\left(\mathrm{dd}, J_{\mathrm{C}-\mathrm{F}}=31.8,24.2 \mathrm{~Hz}, 1 \mathrm{C}\right) .{ }^{19} \mathrm{~F}$ NMR (471 MHz, DMSO- $\left.d_{6}\right) \delta(\mathrm{ppm})-106.5(\mathrm{~d}, J=277.8 \mathrm{~Hz}$, 1F), $-109.5(\mathrm{~d}, J=277.8 \mathrm{~Hz}, 1 \mathrm{~F})$. HRMS (ESI) $\mathrm{m} / \mathrm{z}:[\mathrm{M}+\mathrm{Na}]+$ Calcd for $\mathrm{C}_{18} \mathrm{H}_{10} \mathrm{ClF}_{2} \mathrm{~N}_{3} \mathrm{O}_{5} \mathrm{Na} 444.0169$; Found 444.0173.

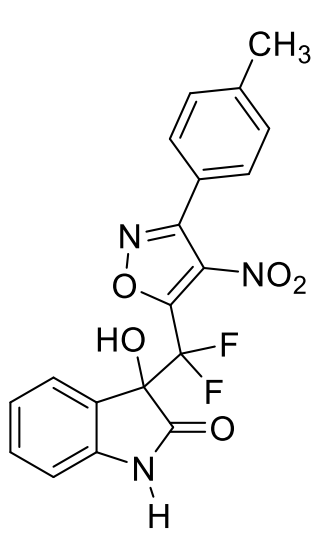

\section{3-(Difluoro(4-nitro-3-(p-tolyl)isoxazol-5-yl)methyl)-3-hydrox} yindolin-2-one (4d): pale yellow solid; mp: decomposed at 196 ${ }^{\circ} \mathrm{C} ; 56.3 \mathrm{mg}, 93 \%$ yield, ${ }^{1} \mathrm{H}$ NMR (400 MHz, DMSO- $\left.d_{6}\right) \delta(\mathrm{ppm})$ $10.90(\mathrm{~s}, 1 \mathrm{H}), 7.75(\mathrm{~s}, 1 \mathrm{H}), 7.55(\mathrm{~s}, 2 \mathrm{H}), 7.40(\mathrm{~s}, 4 \mathrm{H}), 7.11(\mathrm{~s}$, $1 \mathrm{H}), 6.94(\mathrm{~s}, 1 \mathrm{H}), 2.40(\mathrm{~s}, 3 \mathrm{H}) ;{ }^{13} \mathrm{C} \mathrm{NMR}\left(101 \mathrm{MHz}, \mathrm{DMSO}-d_{6}\right)$ $\delta(\mathrm{ppm}) 172.6\left(\mathrm{~d}, J_{\mathrm{C}-\mathrm{F}}=6.3 \mathrm{~Hz}, 1 \mathrm{C}\right), 157.9\left(\mathrm{t}, J_{\mathrm{C}-\mathrm{F}}=31.6 \mathrm{~Hz}\right.$, 1C), $157.5,143.0,141.5,132.3,131.5,129.8,128.3,126.5$, $124.5,122.5,121.6,115.7\left(\mathrm{dd}, J_{\mathrm{C}-\mathrm{F}}=254.9,248.3 \mathrm{~Hz}, 1 \mathrm{C}\right), 110.5$, $77.3\left(\mathrm{dd}, J_{\mathrm{C}-\mathrm{F}}=32.0,25.2 \mathrm{~Hz}, 1 \mathrm{C}\right), 21.1 .{ }^{19} \mathrm{~F} \mathrm{NMR}(471 \mathrm{MHz}$, DMSO- $\left.d_{6}\right) \delta(\mathrm{ppm})-106.3(\mathrm{~d}, J=278.8 \mathrm{~Hz}, 1 \mathrm{~F}),-110.0(\mathrm{~d}, J=$ $278.3 \mathrm{~Hz}, 1 \mathrm{~F}$ ). HRMS (ESI) $\mathrm{m} / \mathrm{z}$ : [M + Na]+ Calcd for $\mathrm{C}_{19} \mathrm{H}_{13} \mathrm{~F}_{2} \mathrm{~N}_{3} \mathrm{O}_{5} \mathrm{Na}$ 424.0712; Found 424.0709 . 


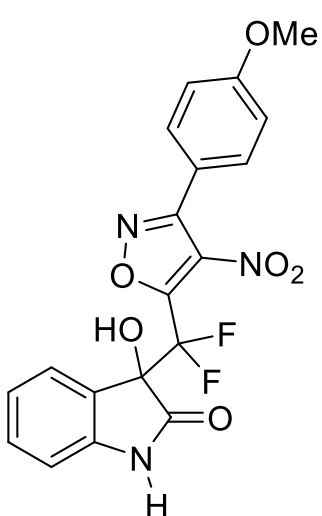

$\mathrm{H}$

3-(Difluoro(3-(4-methoxyphenyl)-4-nitroisoxazol-5-yl)methyl

)-3-hydroxyindolin-2-one (4e): pale yellow solid; mp: decomposed at $168{ }^{\circ} \mathrm{C} ; 52.6 \mathrm{mg}, 84 \%$ yield, ${ }^{1} \mathrm{H}$ NMR $(400 \mathrm{MHz}$, DMSO- $\left.d_{6}\right) \delta(\mathrm{ppm}) 10.88(\mathrm{~s}, 1 \mathrm{H}), 7.73(\mathrm{~s}, 1 \mathrm{H}), 7.60(\mathrm{~d}, J=7.6$ $\mathrm{Hz}, 2 \mathrm{H}), 7.42-7.40(\mathrm{~m}, 2 \mathrm{H}), 7.15-7.10(\mathrm{~m}, 3 \mathrm{H}), 6.94-6.91(\mathrm{~m}$, $1 \mathrm{H}), 3.85(\mathrm{~s}, 3 \mathrm{H}) ;{ }^{13} \mathrm{C} \mathrm{NMR}\left(101 \mathrm{MHz}, \mathrm{DMSO}-d_{6}\right) \delta(\mathrm{ppm})$ $172.6\left(\mathrm{~d}, J_{\mathrm{C}-\mathrm{F}}=6.3 \mathrm{~Hz}, 1 \mathrm{C}\right), 161.6,157.7\left(\mathrm{t}, J_{\mathrm{C}-\mathrm{F}}=32.5 \mathrm{~Hz}, 1 \mathrm{C}\right)$, $157.1,143.0,132.1,131.4,130.0,126.4,124.4,122.4,116.4$, $115.6\left(\mathrm{dd}, J_{\mathrm{C}-\mathrm{F}}=255.5,244.0 \mathrm{~Hz}, 1 \mathrm{C}\right), 114.7,110.4,77.2(\mathrm{dd}$, $\left.J_{\mathrm{C}-\mathrm{F}}=32.2,24.4 \mathrm{~Hz}, 1 \mathrm{C}\right), 55.4 .{ }^{19} \mathrm{~F}$ NMR (471 MHz, DMSO- $d_{6}$ ) $\delta(\mathrm{ppm})-106.3(\mathrm{~d}, J=278.3 \mathrm{~Hz}, 1 \mathrm{~F}),-110.0(\mathrm{~d}, J=278.3 \mathrm{~Hz}, 1 \mathrm{~F})$. HRMS (ESI) m/z: $[\mathrm{M}+\mathrm{Na}]+$ Calcd for $\mathrm{C}_{19} \mathrm{H}_{13} \mathrm{~F}_{2} \mathrm{~N}_{3} \mathrm{O}_{6} \mathrm{Na} 440.0663$; Found 440.0662 .

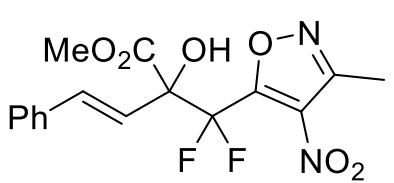

Methyl (E)-2-(difluoro(3-methyl-4-nitroisoxazol-5-yl) methyl) -2-hydroxy-4-phenylbut-3-enoate (5a): white solid; mp: $104-106{ }^{\circ} \mathrm{C} ; 34.3 \mathrm{mg}, 62 \%$ yield, ${ }^{1} \mathrm{H}$ NMR (400 MHz, $\left.\mathrm{CDCl}_{3}\right) \delta(\mathrm{ppm})$ 7.37-7.35 (m, 2H), 7.31-7.21 (m, 3H), 6.84 $(\mathrm{d}, J=8.0 \mathrm{~Hz}, 1 \mathrm{H}), 6.44(\mathrm{dd}, J=15.8,1.8 \mathrm{~Hz}, 1 \mathrm{H}), 4.21(\mathrm{~s}, 1 \mathrm{H}), 3.93(\mathrm{~s}, 3 \mathrm{H}), 2.48(\mathrm{~s}$, $3 \mathrm{H}) ;{ }^{13} \mathrm{C} \mathrm{NMR}\left(101 \mathrm{MHz}, \mathrm{CDCl}_{3}\right) \delta(\mathrm{ppm}) 169.4,159.1$ (dd, $J_{\mathrm{C}-\mathrm{F}}=34.8,33.5 \mathrm{~Hz}, 1 \mathrm{C}$ ), $156.4,134.9\left(\mathrm{~d}, J_{\mathrm{C}-\mathrm{F}}=26.0 \mathrm{~Hz}, 1 \mathrm{C}\right), 132.7,129.9,128.8,128.7,127.1,119.2\left(\mathrm{~d}, J_{\mathrm{C}-\mathrm{F}}\right.$ $=2.9 \mathrm{~Hz}, 1 \mathrm{C}), 115.8\left(\mathrm{dd}, J_{\mathrm{C}-\mathrm{F}}=260.2,257.9 \mathrm{~Hz}, 1 \mathrm{C}\right), 79.4\left(\mathrm{t}, J_{\mathrm{C}-\mathrm{F}}=26.8 \mathrm{~Hz}, 1 \mathrm{C}\right)$, 54.7, 11.2. ${ }^{19} \mathrm{~F}$ NMR $\left(376 \mathrm{MHz}, \mathrm{CDCl}_{3}\right) \delta(\mathrm{ppm})-104.7(\mathrm{~d}, J=272.6 \mathrm{~Hz}, 1 \mathrm{~F}),-106.2$ $(\mathrm{d}, J=272.2 \mathrm{~Hz}, 1 \mathrm{~F})$. HRMS (ESI) $\mathrm{m} / \mathrm{z}:[\mathrm{M}+\mathrm{Na}]+$ Calcd for $\mathrm{C}_{16} \mathrm{H}_{14} \mathrm{~F}_{2} \mathrm{~N}_{2} \mathrm{O}_{6} \mathrm{Na}$ 391.0710; Found 391.0709.

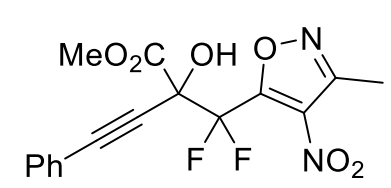

Methyl 2-(difluoro(3-methyl-4-nitroisoxazol-5-yl)methyl)2-hydroxy-4-phenylbut-3-ynoate (5b): yellow oil; $51.1 \mathrm{mg}$, 93\% yield, ${ }^{1} \mathrm{H}$ NMR (400 MHz, $\left.\mathrm{CDCl}_{3}\right) \delta(\mathrm{ppm}) 7.50-7.48(\mathrm{~m}$, 2H), 7.41-7.31 (m, 3H), $4.45(\mathrm{~s}, 1 \mathrm{H}), 4.02(\mathrm{~s}, 3 \mathrm{H}), 2.58(\mathrm{~s}$, $3 \mathrm{H}) ;{ }^{13} \mathrm{C} \mathrm{NMR}\left(101 \mathrm{MHz}, \mathrm{CDCl}_{3}\right) \delta(\mathrm{ppm}) 167.0,158.7\left(\mathrm{dd}, J_{\mathrm{C}-\mathrm{F}}=35.4,32.7 \mathrm{~Hz}, 1 \mathrm{C}\right)$, 156.5, 133.1, 132.1, 129.8, 128.4, 120.3, $114.3\left(\mathrm{dd}, J_{\mathrm{C}-\mathrm{F}}=261.2,258.7 \mathrm{~Hz}, 1 \mathrm{C}\right), 89.0$, $79.3\left(\mathrm{~d}, J_{\mathrm{C}-\mathrm{F}}=6.2 \mathrm{~Hz}, 1 \mathrm{C}\right), 74.1\left(\mathrm{dd}, J_{\mathrm{C}-\mathrm{F}}=31.6,29.1 \mathrm{~Hz}, 1 \mathrm{C}\right), 55.3,11.2 .{ }^{19} \mathrm{~F}$ NMR $\left(376 \mathrm{MHz}, \mathrm{CDCl}_{3}\right) \delta(\mathrm{ppm})-103.6(\mathrm{~d}, J=268.8 \mathrm{~Hz}, 1 \mathrm{~F}),-107.1(\mathrm{~d}, J=268.5 \mathrm{~Hz}, 1 \mathrm{~F})$. HRMS (ESI) m/z: [M + Na]+ Calcd for $\mathrm{C}_{16} \mathrm{H}_{12} \mathrm{~F}_{2} \mathrm{~N}_{2} \mathrm{O}_{6} \mathrm{Na} 389.0553$; Found 389.0551.

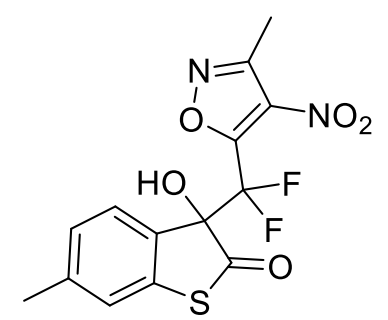

3-(difluoro(3-methyl-4-nitroisoxazol-5-yl)methyl)-3-hydrox y-6-methylbenzo[b]thiophen-2(3H)-one (5c): yellow oil; $42.8 \mathrm{mg}, 81 \%$ yield, ${ }^{1} \mathrm{H}$ NMR (400 MHz, DMSO- $\left.d_{6}\right) \delta(\mathrm{ppm})$ $8.16(\mathrm{~s}, 1 \mathrm{H}), 7.49(\mathrm{~s}, 1 \mathrm{H}), 7.37(\mathrm{~d}, J=8.0 \mathrm{~Hz}, 1 \mathrm{H}), 7.24(\mathrm{~s}$, 1H), $2.53(\mathrm{~s}, 3 \mathrm{H}), 2.37(\mathrm{~s}, 3 \mathrm{H}) ;{ }^{13} \mathrm{C} \mathrm{NMR}(101 \mathrm{MHz}$, DMSO- $\left.d_{6}\right) \delta(\mathrm{ppm}) 201.1,157.1\left(\mathrm{t}, J_{\mathrm{C}-\mathrm{F}}=34.0 \mathrm{~Hz}, 1 \mathrm{C}\right), 156.9$, $142.2,133.8,133.4,128.3,128.0,127.7,124.4,115.2\left(\mathrm{dd}, J_{\mathrm{C}-\mathrm{F}}=262.0,251.0 \mathrm{~Hz}, 1 \mathrm{C}\right)$, $84.8\left(\mathrm{dd}, J_{\mathrm{C}-\mathrm{F}}=30.0,24.0 \mathrm{~Hz}, 1 \mathrm{C}\right), 21.1,10.7 .{ }^{19} \mathrm{~F} \mathrm{NMR}\left(376 \mathrm{MHz}, \mathrm{DMSO}-d_{6}\right) \delta$ 
(ppm) -105.7 (d, $J=275.2 \mathrm{~Hz}, 1 \mathrm{~F}),-107.8(\mathrm{~d}, J=275.2 \mathrm{~Hz}, 1 \mathrm{~F})$. HRMS (ESI) m/z: $[\mathrm{M}+\mathrm{H}]+$ Calcd for $\mathrm{C}_{14} \mathrm{H}_{11} \mathrm{~F}_{2} \mathrm{~N}_{2} \mathrm{O}_{5} \mathrm{~S}$ 357.0356; Found 357.0365.

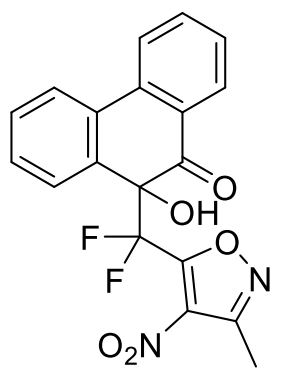

10-(difluoro(3-methyl-4-nitroisoxazol-5-yl)methyl)-10-hydroxyp henanthren-9(10H)-one (5d): pale yellow solid; mp: 190-192 ${ }^{\circ} \mathrm{C}$; $50.7 \mathrm{mg}, 88 \%$ yield, ${ }^{1} \mathrm{H}$ NMR (400 MHz, DMSO- $\left.d_{6}\right) \delta$ (ppm) 8.18 $(\mathrm{d}, J=8.0 \mathrm{~Hz}, 1 \mathrm{H}), 8.12(\mathrm{~d}, J=8.0 \mathrm{~Hz}, 1 \mathrm{H}), 7.83-7.81(\mathrm{~m}, 2 \mathrm{H})$, 7.59-7.50 (m, 3H), 7.47-7.42 (m, 2H), $2.45(\mathrm{~s}, 3 \mathrm{H}) ;{ }^{13} \mathrm{C}$ NMR $(101$ $\left.\mathrm{MHz}, \mathrm{DMSO}-d_{6}\right) \delta(\mathrm{ppm}) 195.6,157.7\left(\mathrm{t}, J_{\mathrm{C}-\mathrm{F}}=34.0 \mathrm{~Hz}, 1 \mathrm{C}\right)$, $156.7,137.2,136.0,133.0,132.1,131.5,130.5,128.94,128.93$, $128.9,127.2,124.7,123.7,115.9\left(\mathrm{dd}, J_{\mathrm{C}-\mathrm{F}}=260.0,258.0 \mathrm{~Hz}, 1 \mathrm{C}\right), 79.8\left(\mathrm{dd}, J_{\mathrm{C}-\mathrm{F}}=\right.$ 26.5, 23.5 Hz, 1C), 10.7. ${ }^{19} \mathrm{~F}$ NMR (376 MHz, DMSO- $\left.d_{6}\right) \delta(\mathrm{ppm})-103.1(\mathrm{~d}, J=$ $265.5 \mathrm{~Hz}, 1 \mathrm{~F}),-104.9$ (d, $J=265.5 \mathrm{~Hz}, 1 \mathrm{~F})$. HRMS (ESI) m/z: [M + H]+ Calcd for $\mathrm{C}_{19} \mathrm{H}_{13} \mathrm{~F}_{2} \mathrm{~N}_{2} \mathrm{O}_{5}$ 387.0793; Found 387.0787.

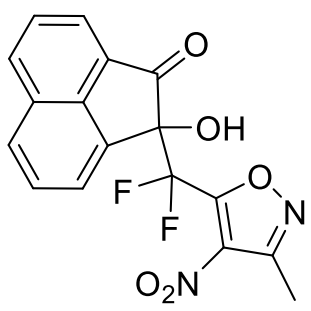

2-(difluoro(3-methyl-4-nitroisoxazol-5-yl)methyl)-2-hydroxya cenaphthylen-1(2H)-one (5e): white solid; mp: 162-163 ${ }^{\circ} \mathrm{C}$; $51.5 \mathrm{mg}, 95 \%$ yield, ${ }^{1} \mathrm{H}$ NMR (400 MHz, DMSO- $\left.d_{6}\right) \delta(\mathrm{ppm})$ $8.42(\mathrm{~d}, J=8.0 \mathrm{~Hz}, 1 \mathrm{H}), 8.20(\mathrm{~d}, J=8.0 \mathrm{~Hz}, 1 \mathrm{H}), 8.05(\mathrm{~d}, J=8.0$ $\mathrm{Hz}, 1 \mathrm{H}), 7.91(\mathrm{t}, J=8.0 \mathrm{~Hz}, 1 \mathrm{H}), 7.87-7.80(\mathrm{~m}, 3 \mathrm{H}), 2.57(\mathrm{~s}, 3 \mathrm{H})$; ${ }^{13} \mathrm{C}$ NMR $\left(101 \mathrm{MHz}, \mathrm{DMSO}-d_{6}\right) \delta(\mathrm{ppm}) 198.1,158.0\left(\mathrm{t}, J_{\mathrm{C}-\mathrm{F}}=\right.$ $34.5 \mathrm{~Hz}, 1 \mathrm{C}), 156.8,142.1,133.5,133.3,132.9,130.2,130.0,129.0,128.9,127.1$, $123.4,122.7,116.0\left(\mathrm{dd}, J_{\mathrm{C}-\mathrm{F}}=256.5,249.5 \mathrm{~Hz}, 1 \mathrm{C}\right), 80.5\left(\mathrm{dd}, J_{\mathrm{C}-\mathrm{F}}=31.0,24.0 \mathrm{~Hz}\right.$, 1C), 10.7. ${ }^{19} \mathrm{~F}$ NMR $\left(376 \mathrm{MHz}, \mathrm{DMSO}-d_{6}\right) \delta(\mathrm{ppm})-104.6(\mathrm{~d}, J=277.9 \mathrm{~Hz}, 1 \mathrm{~F})$, $-106.6(\mathrm{~d}, J=277.9 \mathrm{~Hz}, 1 \mathrm{~F})$. HRMS (ESI) $\mathrm{m} / \mathrm{z}$ : $[\mathrm{M}+\mathrm{H}]+$ Calcd for $\mathrm{C}_{17} \mathrm{H}_{11} \mathrm{~F}_{2} \mathrm{~N}_{2} \mathrm{O}_{5}$ 361.0636; Found 361.0626.

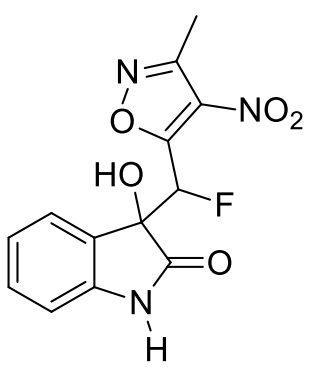

\section{3-((4-nitro-3-methylisoxazol-5-yl)fluoromethyl)-3-hydroxyind} olin-2-one (8) : yellow solid; $\mathrm{mp}: 180-185{ }^{\circ} \mathrm{C} ; 20.0 \mathrm{mg}, 65 \%$ yield, $97: 3 \mathrm{dr}$, this compound is relatively unstable during column chromatography. ${ }^{1} \mathrm{H}$ NMR (400 MHz, DMSO-d $) \delta(\mathrm{ppm}) 10.72$ (s, 1H), $7.32(\mathrm{t}, J=7.6 \mathrm{~Hz} 1 \mathrm{H}), 7.08-7.01(\mathrm{~m}, 2 \mathrm{H}), 6.97(\mathrm{~s}, 1 \mathrm{H})$, $6.90(\mathrm{~d}, J=8.0 \mathrm{~Hz} 1 \mathrm{H}), 6.38(\mathrm{~d}, J=42.8 \mathrm{~Hz}, 1 \mathrm{H}), 2.55$ (s, 3H); ${ }^{13} \mathrm{C}$ NMR (101 MHz, DMSO- $\left.d_{6}\right) \delta(\mathrm{ppm}) 174.8\left(\mathrm{~d}, J_{\mathrm{C}-\mathrm{F}}=3.4 \mathrm{~Hz}\right.$, $1 \mathrm{C}), 164.6\left(\mathrm{~d}, J_{\mathrm{C}-\mathrm{F}}=19.7 \mathrm{~Hz}, 1 \mathrm{C}\right), 155.6,142.3,130.5,126.8,125.8,121.8,110.1$, $86.3\left(\mathrm{~d}, J_{\mathrm{C}-\mathrm{F}}=183.2 \mathrm{~Hz}, 1 \mathrm{C}\right), 76.2\left(\mathrm{~d}, J_{\mathrm{C}-\mathrm{F}}=25.3 \mathrm{~Hz}, 1 \mathrm{C}\right), 10.9 .{ }^{19} \mathrm{~F}$ NMR $(376 \mathrm{MHz}$, DMSO- $\left.d_{6}\right) \delta(\mathrm{ppm})-200.7(\mathrm{~d}, J=421.1 \mathrm{~Hz}, 1 \mathrm{~F})$. HRMS (ESI) m/z: [M - H]- Calcd for $\mathrm{C}_{10} \mathrm{H}_{9} \mathrm{FN}_{3} \mathrm{O}_{5} 306.0532$; Found 306.0532 . 
2.3 Control reactions.

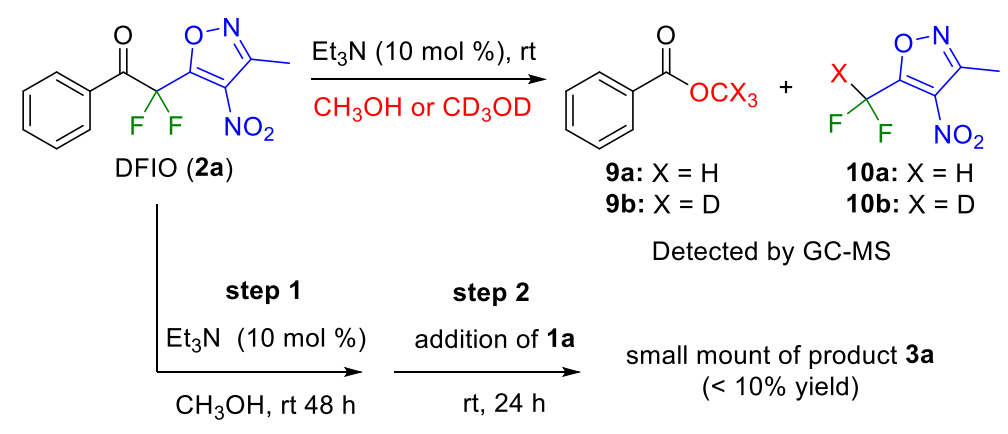

\section{GC-MS Spectrums}
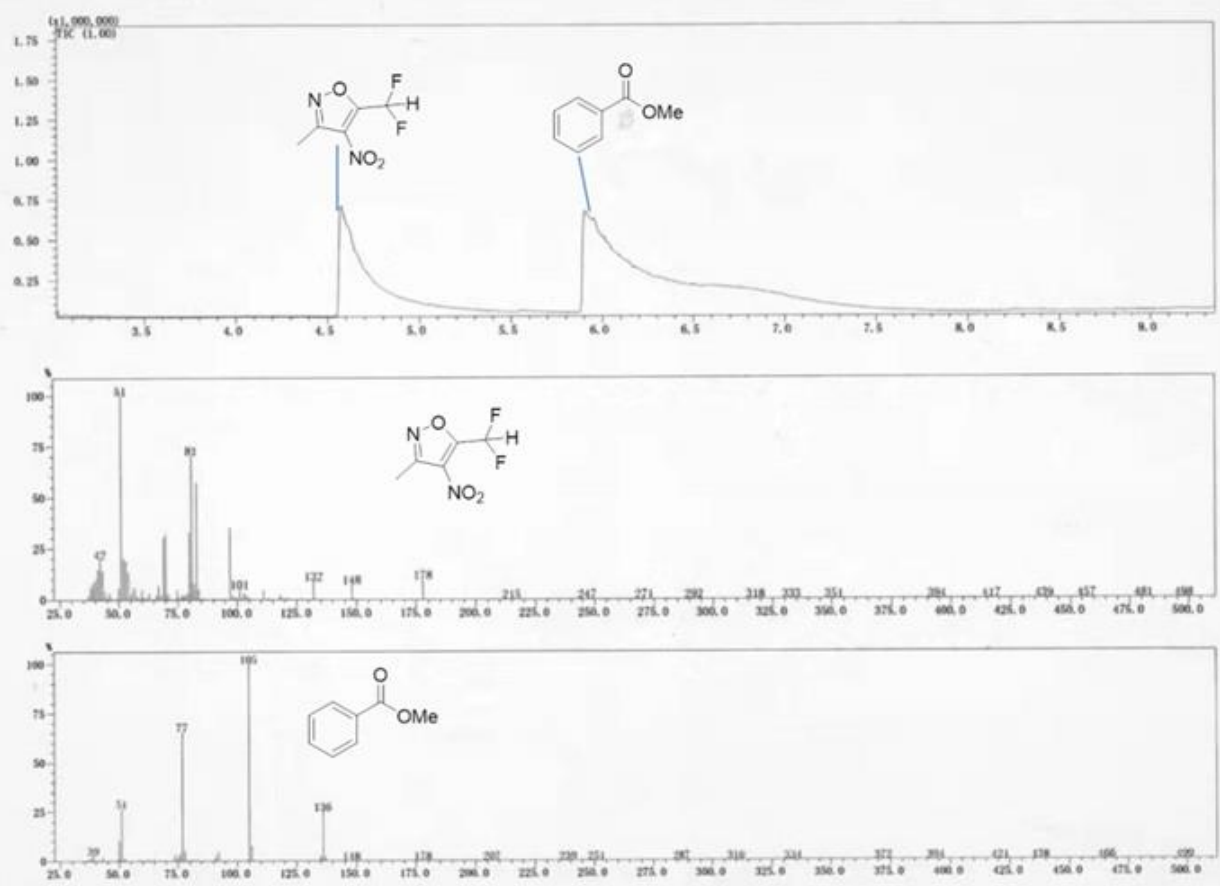

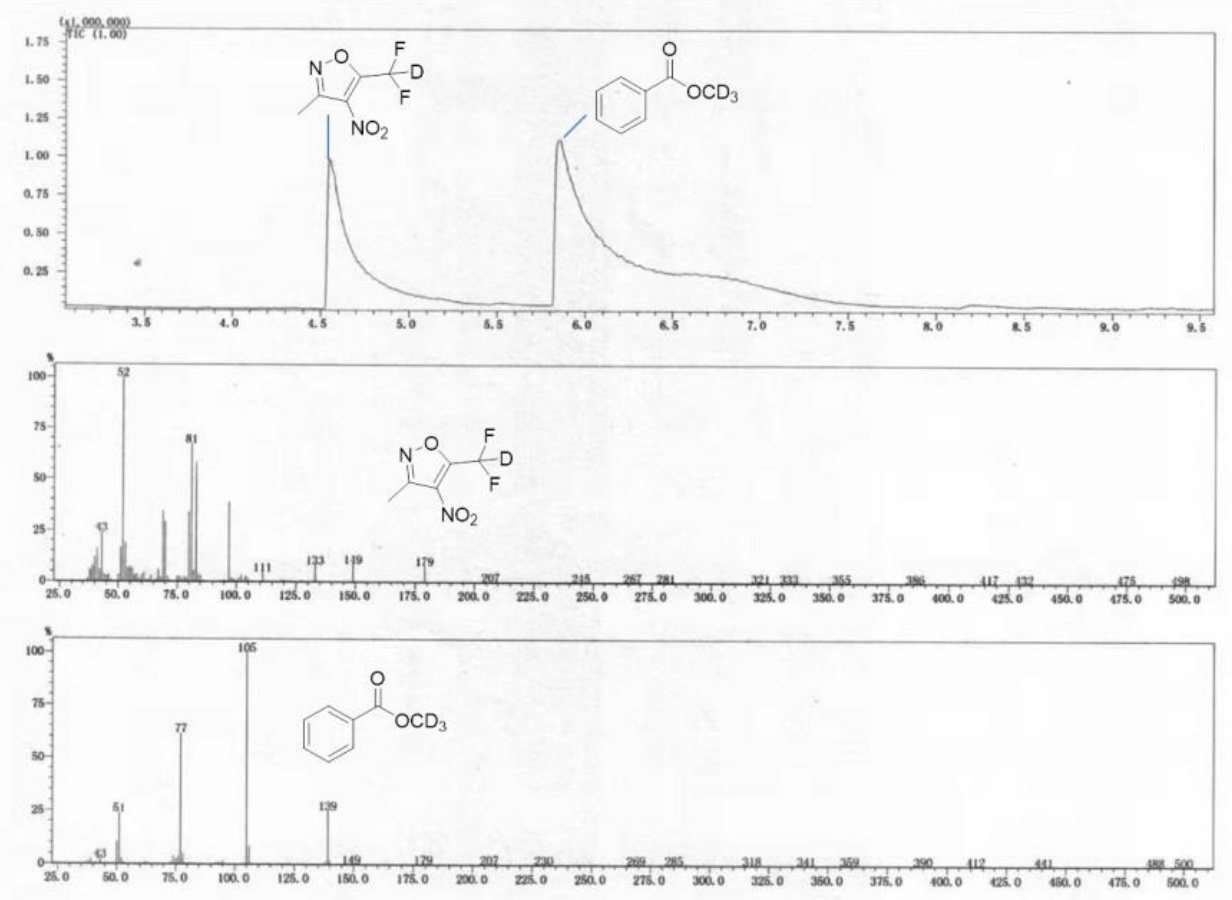

\subsection{Reaction procedure for gram-scale synthesis of $3 \mathrm{a}$.}<smiles>O=C1Nc2ccccc2C1=O</smiles>

$1 \mathrm{a}$<smiles>Cc1noc(C(F)(F)C(=O)c2ccccc2)c1[N+](=O)[O-]</smiles>

$2 a$

$0.736 \mathrm{~g}, 5.0 \mathrm{mmol} \quad 1.693 \mathrm{~g}, 6.0 \mathrm{mmol}$

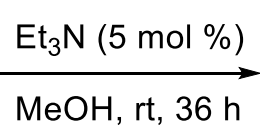

$\mathrm{MeOH}, \mathrm{rt}, 36 \mathrm{~h}$<smiles>Cc1noc(C(F)(F)C2(O)C(=O)Nc3ccccc32)c1[N+](=O)[O-]</smiles>

$3 a$

$1.615 \mathrm{~g}, 99 \%$ yield

Isatin $1 \mathrm{a}(0.736 \mathrm{~g}, 5.0 \mathrm{mmol})$, difluoroisoxazolacetophenone $\mathbf{2 a}(1.693 \mathrm{~g}, 6.0$ mmol) and $5 \mathrm{~mL} \mathrm{CH}_{3} \mathrm{OH}$ were stirred at room temperature, then TEA ( $5 \mathrm{~mol} \%, 0.25$ mmol) was added. After stirred for 36 hours, the reaction mixture was concentrated, and the product 3a was purified by silica gel chromatography (eluent: PE/acetone $=$ $3 / 1, \mathrm{v} / \mathrm{v})$.

\subsection{Transformations of $3 a$.}<smiles>Cc1noc(C(F)(F)C2(O)C(=O)Nc3ccccc32)c1N</smiles>

11

$99 \%$ yield

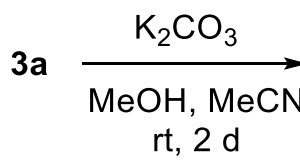<smiles>O=C(O)C(F)(F)C1(O)C(=O)Nc2ccccc21</smiles>

12

$83 \%$ yield 
To the solution of 3a $(65.1 \mathrm{mg}, 0.2 \mathrm{mmol}, 1.0$ equiv.) in acetic acid ( $5 \mathrm{~mL})$, zinc dust $(0.47 \mathrm{~g}, 3.13 \mathrm{mmol}, 15.6$ equiv. $)$ was added slowly. After stirred for $1.5 \mathrm{~h}$ at room temperature, the mixture was filtered over celite, diluted with ethyl acetate $(10 \mathrm{~mL})$, and then washed with saturated sodium bicarbonate solution $(2 \times 10 \mathrm{~mL})$. The combined organic phase was dried over $\mathrm{Na}_{2} \mathrm{SO}_{4}$ and concentrated in vacuo to give a yellow solid

3-((4-amino-3-methylisoxazol-5-yl)difluoromethyl)-3-hydroxyindolin-2-one 11: mp: decomposed at $160{ }^{\circ} \mathrm{C} ; 58.4 \mathrm{mg}, 99 \%$ yield, ${ }^{1} \mathrm{H}$ NMR (400 MHz, DMSO- $\left.d_{6}\right) \delta$ (ppm) $10.62(\mathrm{~s}, 1 \mathrm{H}), 7.44$ (s, 1H), 7.29 (t, $J=7.0 \mathrm{~Hz}, 1 \mathrm{H}), 7.15$ (d, $J=6.8 \mathrm{~Hz}, 1 \mathrm{H})$, $6.98(\mathrm{~d}, J=6.8 \mathrm{~Hz}, 1 \mathrm{H}), 6.83(\mathrm{~d}, J=7.2 \mathrm{~Hz}, 1 \mathrm{H}), 4.51(\mathrm{~s}, 2 \mathrm{H}), 2.14(\mathrm{~s}, 3 \mathrm{H}) ;{ }^{13} \mathrm{C} \mathrm{NMR}$ $\left(101 \mathrm{MHz}, \mathrm{DMSO}-d_{6}\right) \delta(\mathrm{ppm}) 173.4,154.2,142.8,139.4\left(\mathrm{t}, J_{\mathrm{C}-\mathrm{F}}=32.1 \mathrm{~Hz}, 1 \mathrm{C}\right)$, $130.7,128.1,125.9,125.8,121.9,117.3\left(\mathrm{t}, J_{\mathrm{C}-\mathrm{F}}=248.3 \mathrm{~Hz}, 1 \mathrm{C}\right), 110.0,77.5\left(\mathrm{t}, J_{\mathrm{C}-\mathrm{F}}\right.$ $=28.7 \mathrm{~Hz}, 1 \mathrm{C}), 9.0 .{ }^{19} \mathrm{~F}$ NMR $\left(376 \mathrm{MHz}, \mathrm{DMSO}-d_{6}\right) \delta(\mathrm{ppm})-107.1(\mathrm{~d}, J=271.9 \mathrm{~Hz}$, $1 \mathrm{~F}),-108.5(\mathrm{~d}, J=272.2 \mathrm{~Hz}, 1 \mathrm{~F})$. HRMS (ESI) $\mathrm{m} / \mathrm{z}:[\mathrm{M}+\mathrm{H}]+$ Calcd for $\mathrm{C}_{13} \mathrm{H}_{12} \mathrm{~F}_{2} \mathrm{~N}_{3} \mathrm{O}_{3}$ 296.0847; Found 296.0841.

3a (65.0 mg, $0.2 \mathrm{mmol}, 1.0$ equiv.), potassium carbonate $(69.1 \mathrm{mg}, 0.5 \mathrm{mmol}, 2.5$ equiv.), $\mathrm{CH}_{3} \mathrm{OH}$ (19.2 mg, $0.6 \mathrm{mmol}, 3.0$ equiv.) and acetonitrile (1 mL) were stirred at room temperature for 2 days. After completion of the reaction, the mixture was treated with $1 \mathrm{M}$ hydrochloric acid and concentrated under reduced pressure. The solid mixture was washed several times with methanol to give a yellow solid of 2,2-difluoro-2-(3-hydroxy-2-oxoindolin-3-yl)acetic acid 12: $\mathrm{mp}$ : decomposed at $280{ }^{\circ} \mathrm{C}$; $40.5 \mathrm{mg}, 83 \%$ yield, ${ }^{1} \mathrm{H}$ NMR (400 MHz, DMSO- $\left.d_{6}\right) \delta(\mathrm{ppm}) 10.38(\mathrm{~s}, 1 \mathrm{H})$, $8.80(\mathrm{~s}, 1 \mathrm{H}), 7.30(\mathrm{~d}, J=6.8 \mathrm{~Hz}, 1 \mathrm{H}), 7.25(\mathrm{t}, J=7.0 \mathrm{~Hz}, 1 \mathrm{H}), 6.96-6.92(\mathrm{~m}, 1 \mathrm{H})$, $6.80(\mathrm{~d}, J=7.2 \mathrm{~Hz}, 1 \mathrm{H}) ;{ }^{13} \mathrm{C}$ NMR $\left(101 \mathrm{MHz}, \mathrm{DMSO}-d_{6}\right) \delta(\mathrm{ppm}) 175.2,164.5,143.2$, $129.9,126.9,126.0,121.5,112.6\left(\mathrm{t}, J_{\mathrm{C}-\mathrm{F}}=262.0 \mathrm{~Hz}, 1 \mathrm{C}\right), 109.7,76.8\left(\mathrm{t}, J_{\mathrm{C}-\mathrm{F}}=26.4\right.$ $\mathrm{Hz}, 1 \mathrm{C}) .{ }^{19} \mathrm{~F}$ NMR (376 MHz, DMSO- $\left.d_{6}\right) \delta$ (ppm) -114.5 (s, 2F). HRMS (ESI) m/z: [M - H]- Calcd for $\mathrm{C}_{10} \mathrm{H}_{6} \mathrm{~F}_{2} \mathrm{NO}_{4}$ 242.0270; Found 242.0265. 


\section{Crystal structure}
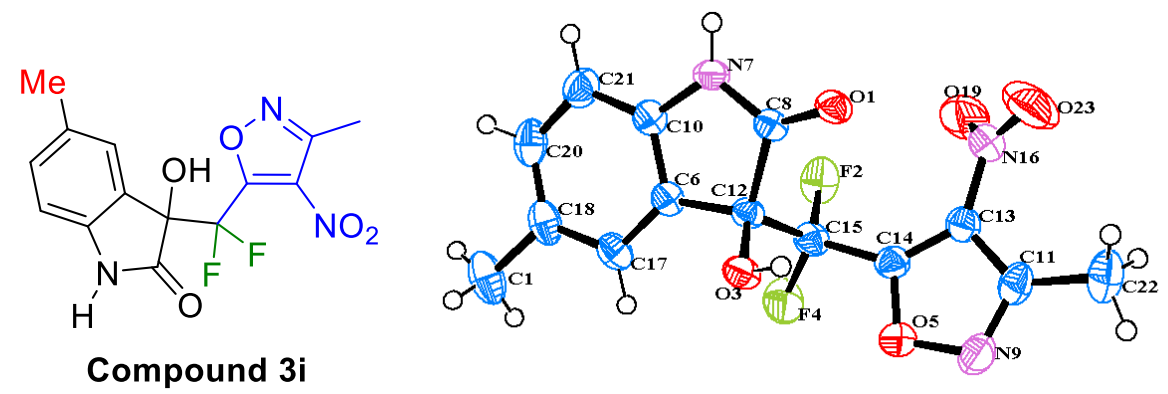

Figure S1: Molecular structure of $\mathbf{3 i}$ with $50 \%$ probability ellipsoids.

Synthesis was carried out by reaction of $\mathbf{1 i}(0.15 \mathrm{mmol})$ and $\mathbf{2 a}(0.20 \mathrm{mmol})$ in $\mathrm{MeOH}$ for $24 \mathrm{~h}$ in a reaction tube. Single crystals suitable for X-ray analysis were grown from ethyl acetate solution of $\mathbf{3 i}$ by slow vapor diffusion of n-hexane. Single-crystal X-ray diffraction data were collected on a SuperNova, Dual, $\mathrm{Cu}$ at zero, EOS diffractometer. The crystal was kept at 293.15(10)K during data collection.

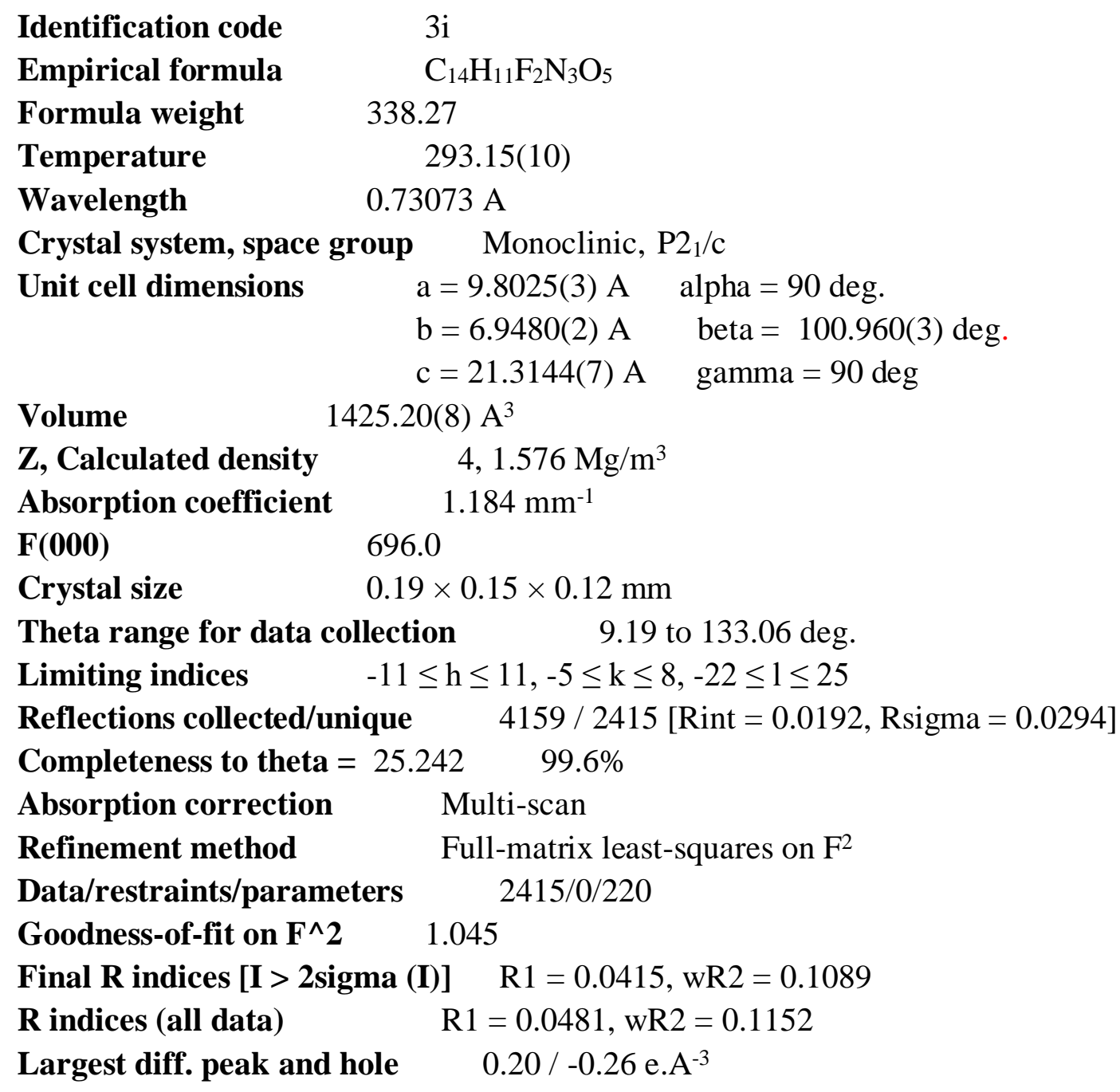




\section{NMR spectrum}

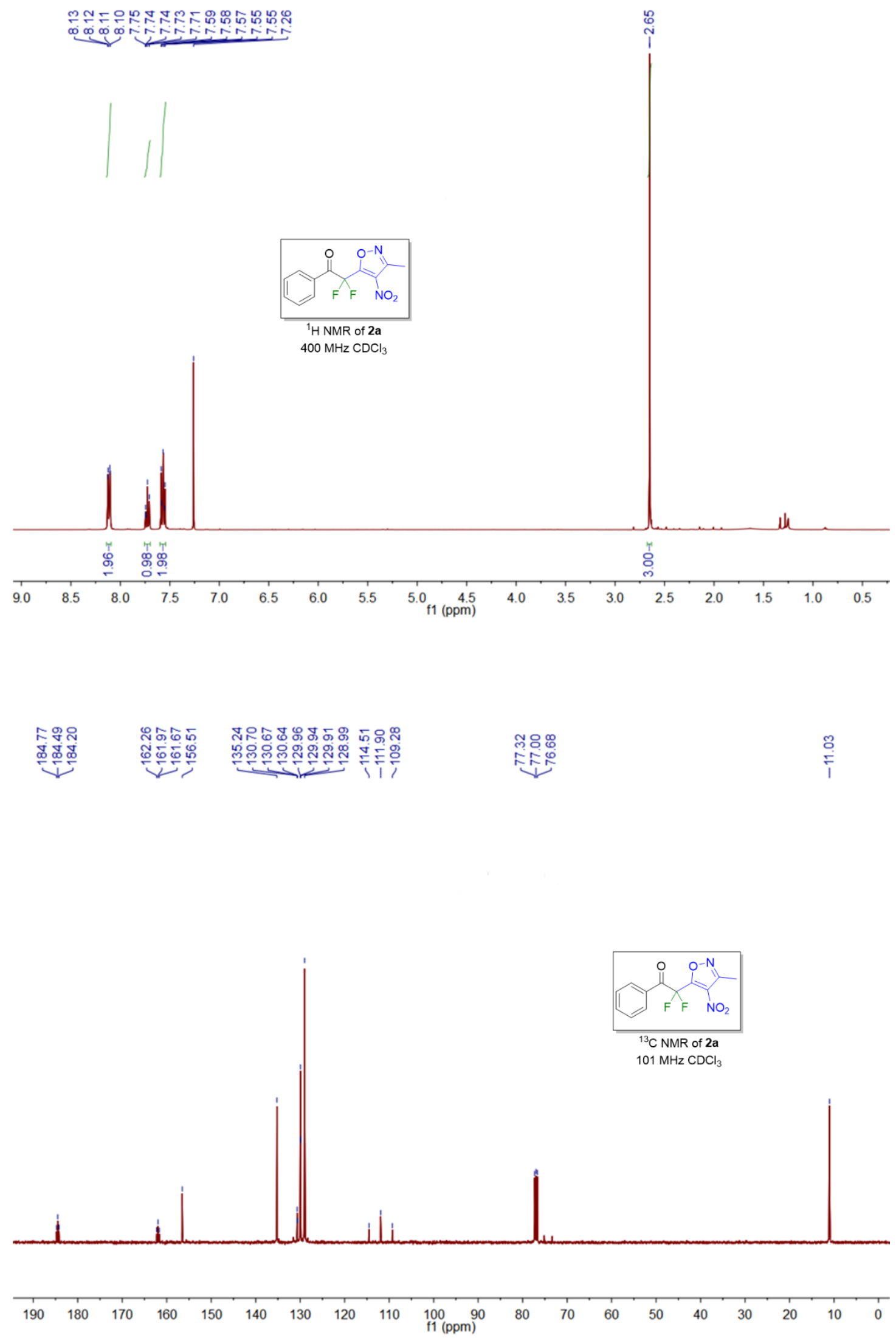



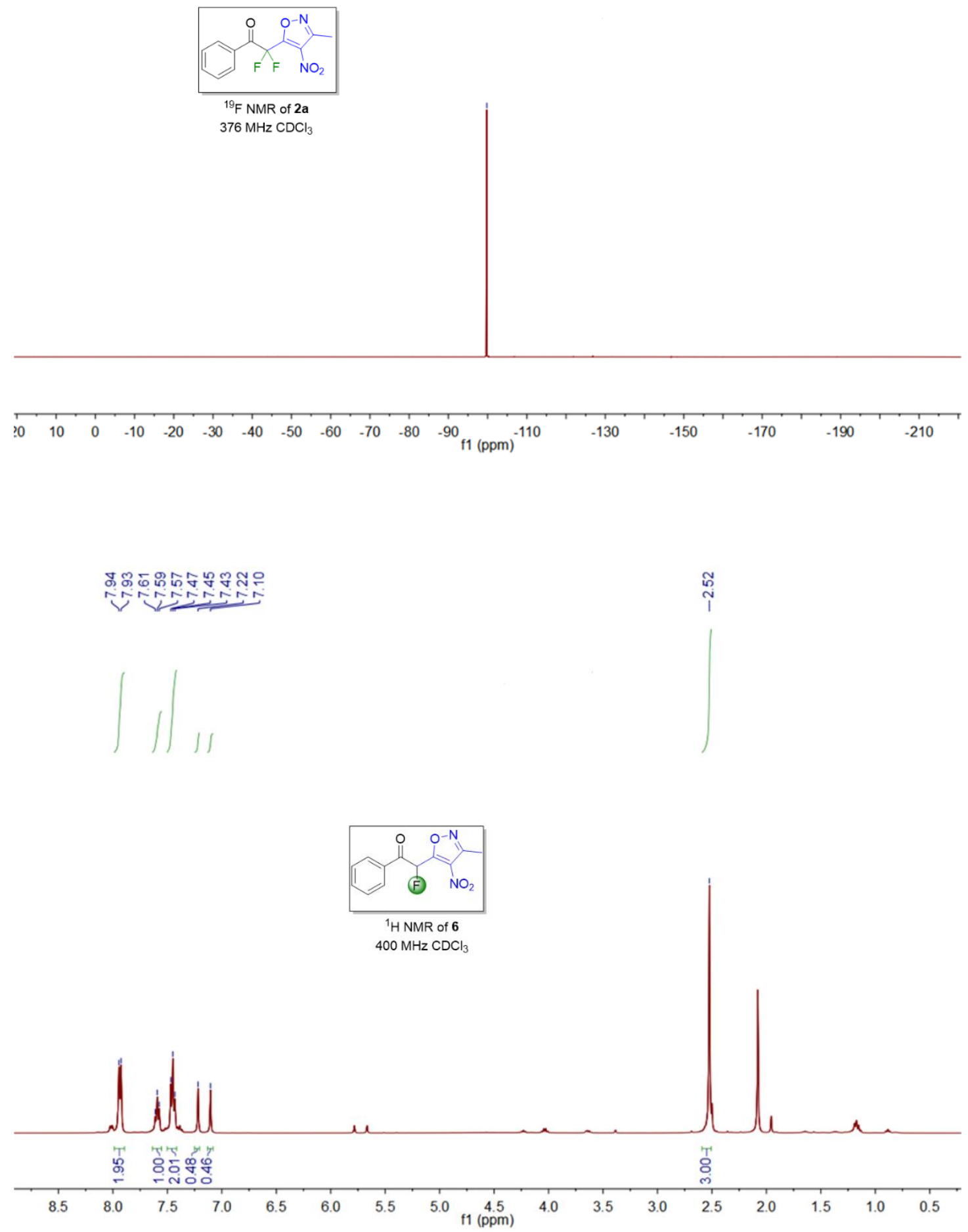


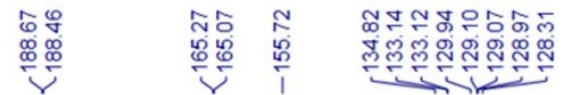

퓨융

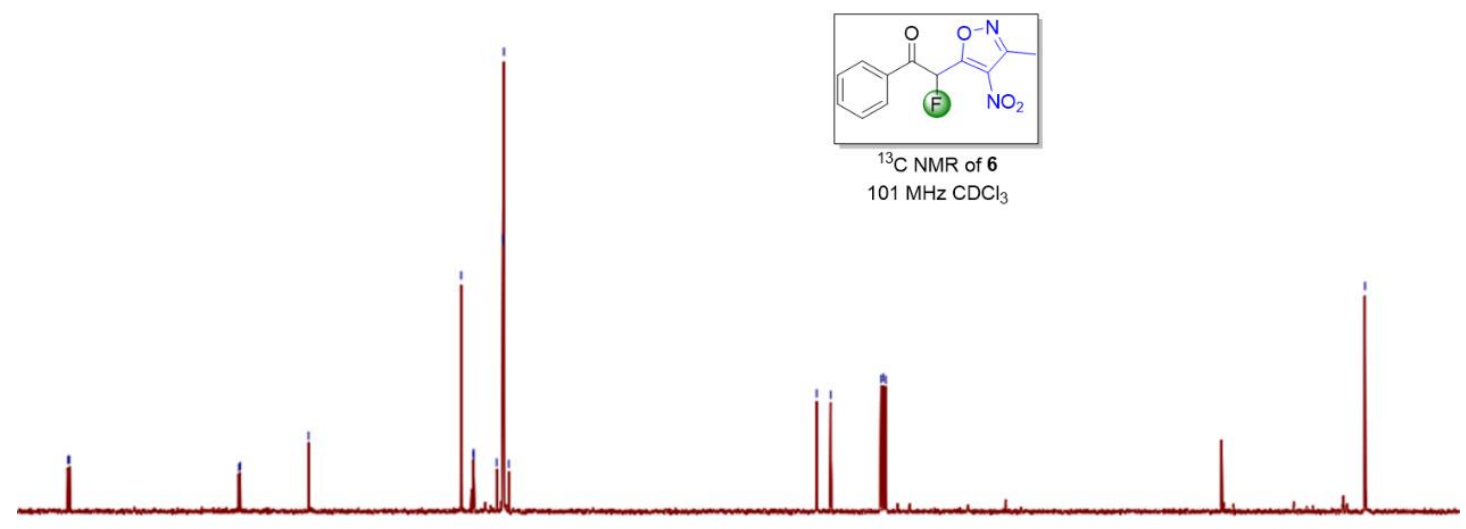

$\begin{array}{llllllllllllllllllll}190 & 180 & 170 & 160 & 150 & 140 & 130 & 120 & 110 & \begin{array}{l}100 \\ \mathrm{f} 1(\mathrm{ppm})\end{array} & 90 & 80 & 70 & 60 & 50 & 40 & 30 & 20 & 10 & 0\end{array}$

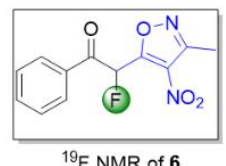

$376 \mathrm{MHz} \mathrm{CDCl}_{3}$ 

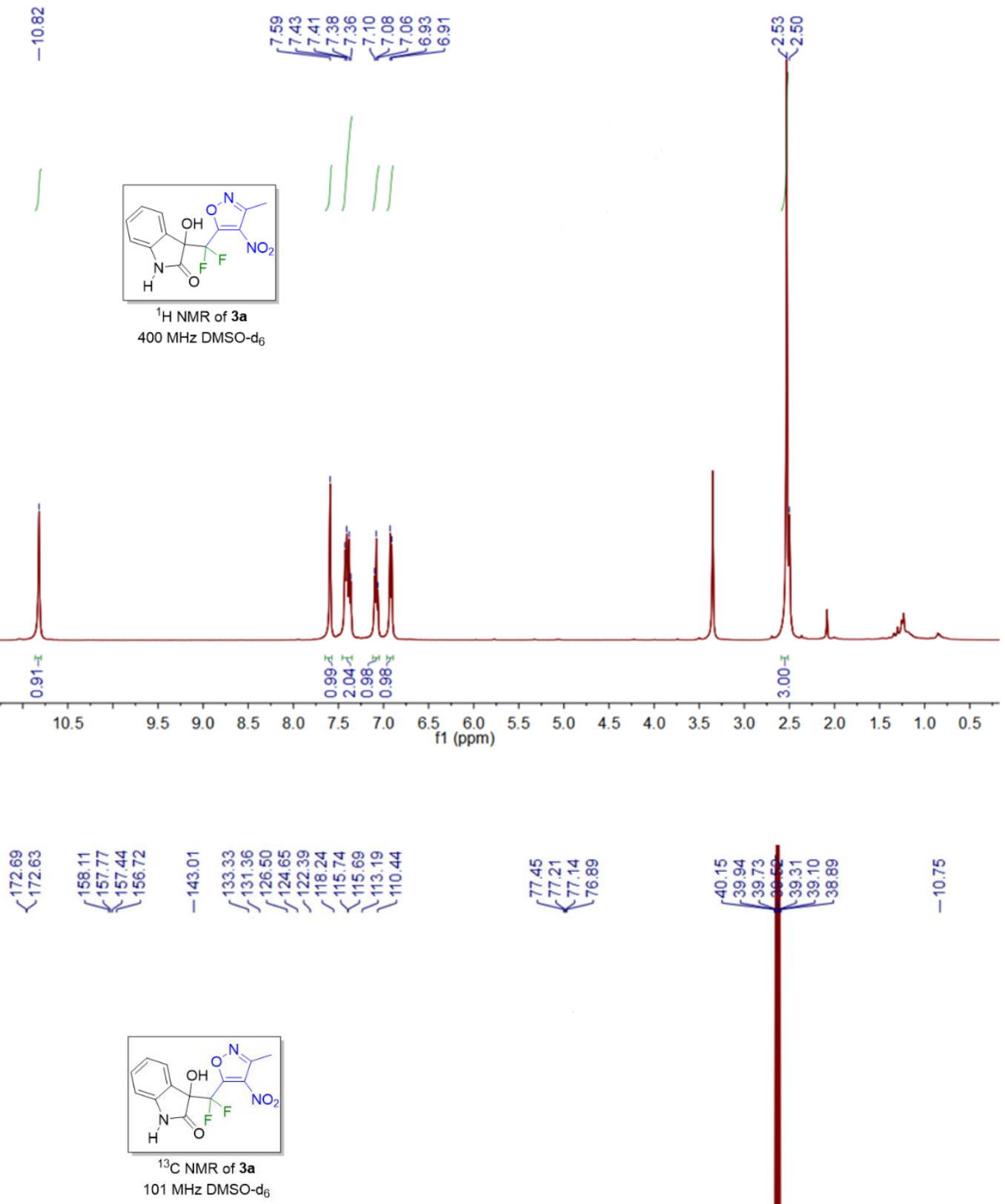

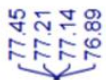

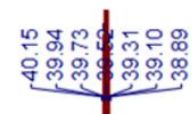

$\frac{n}{\text { 웅 }}$

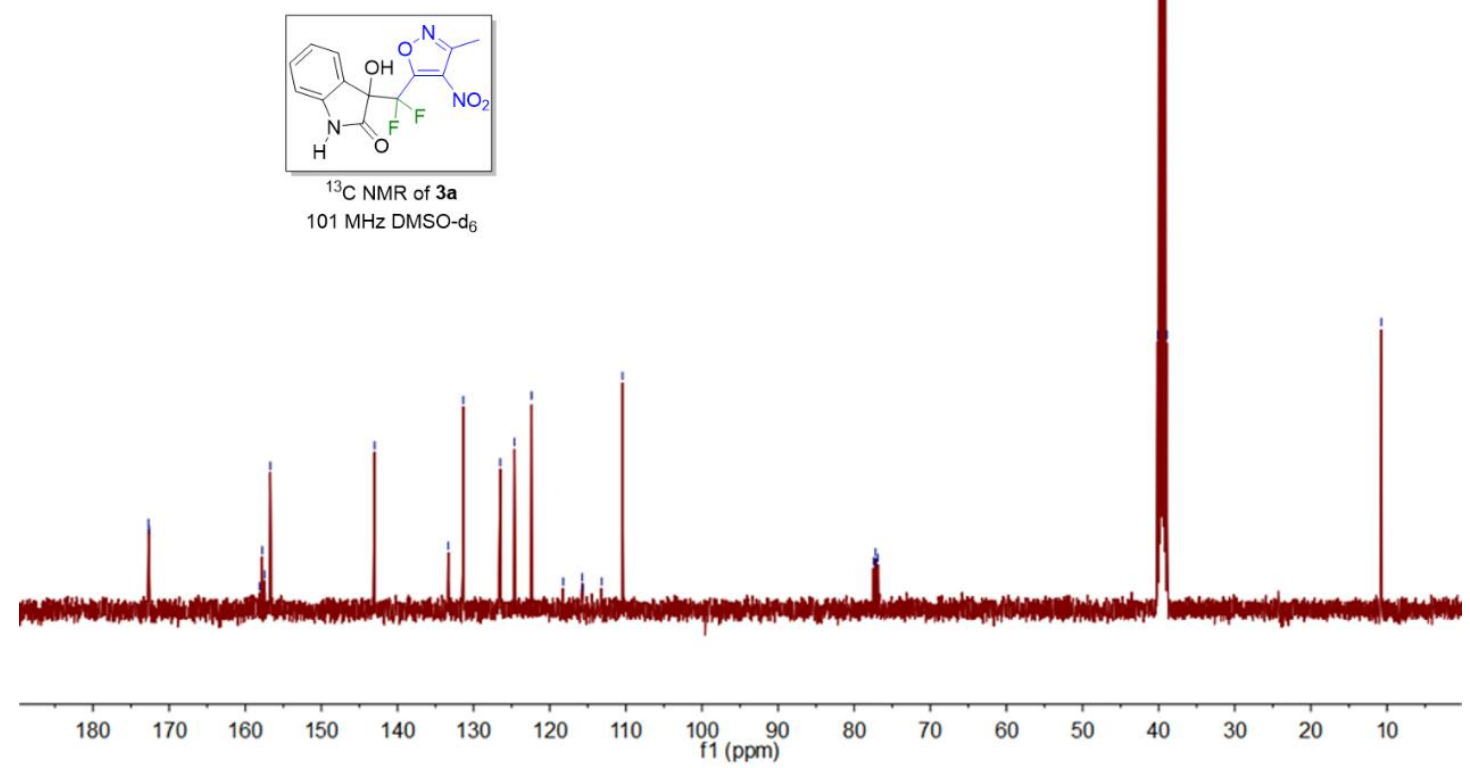



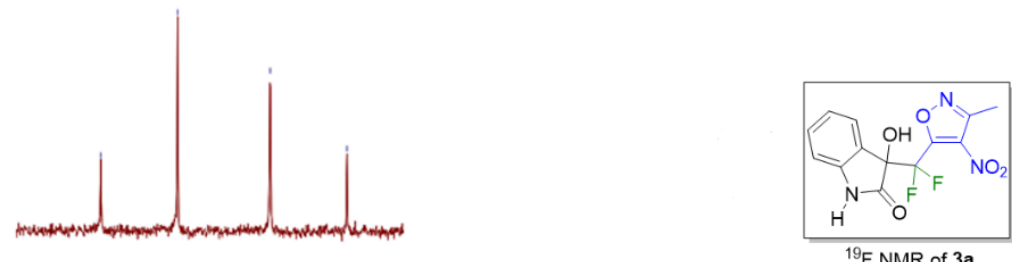

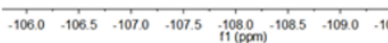

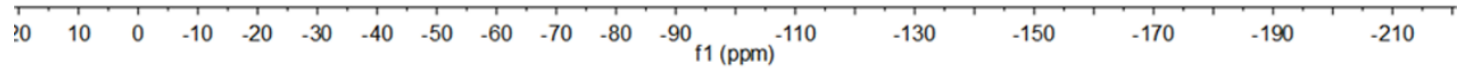

\begin{tabular}{l}
$\infty$ \\
$\infty$ \\
0 \\
\hline
\end{tabular}

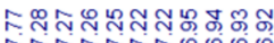

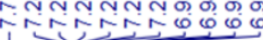

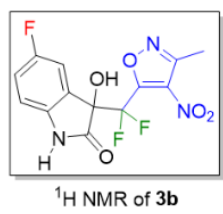

$400 \mathrm{MHz}$ DMSO-d

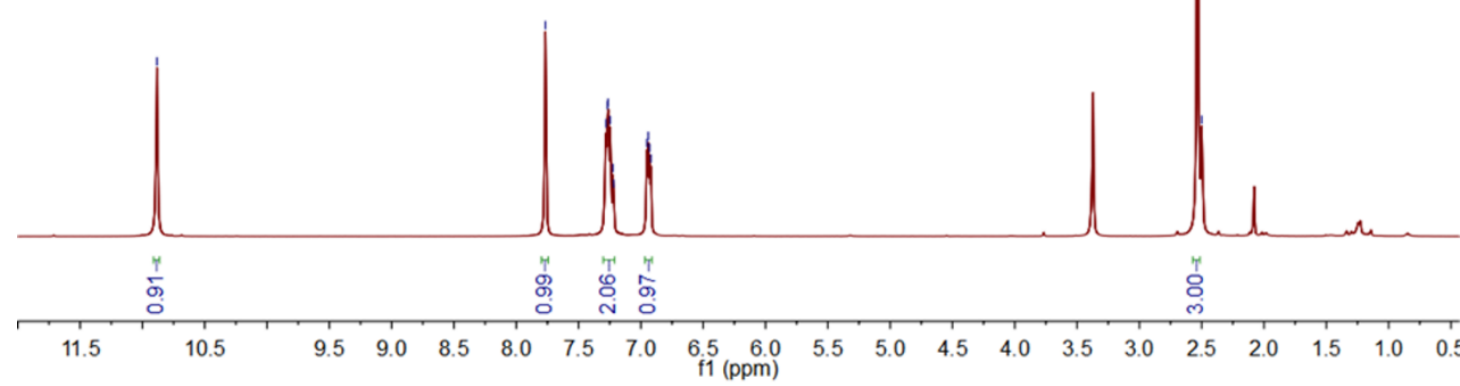




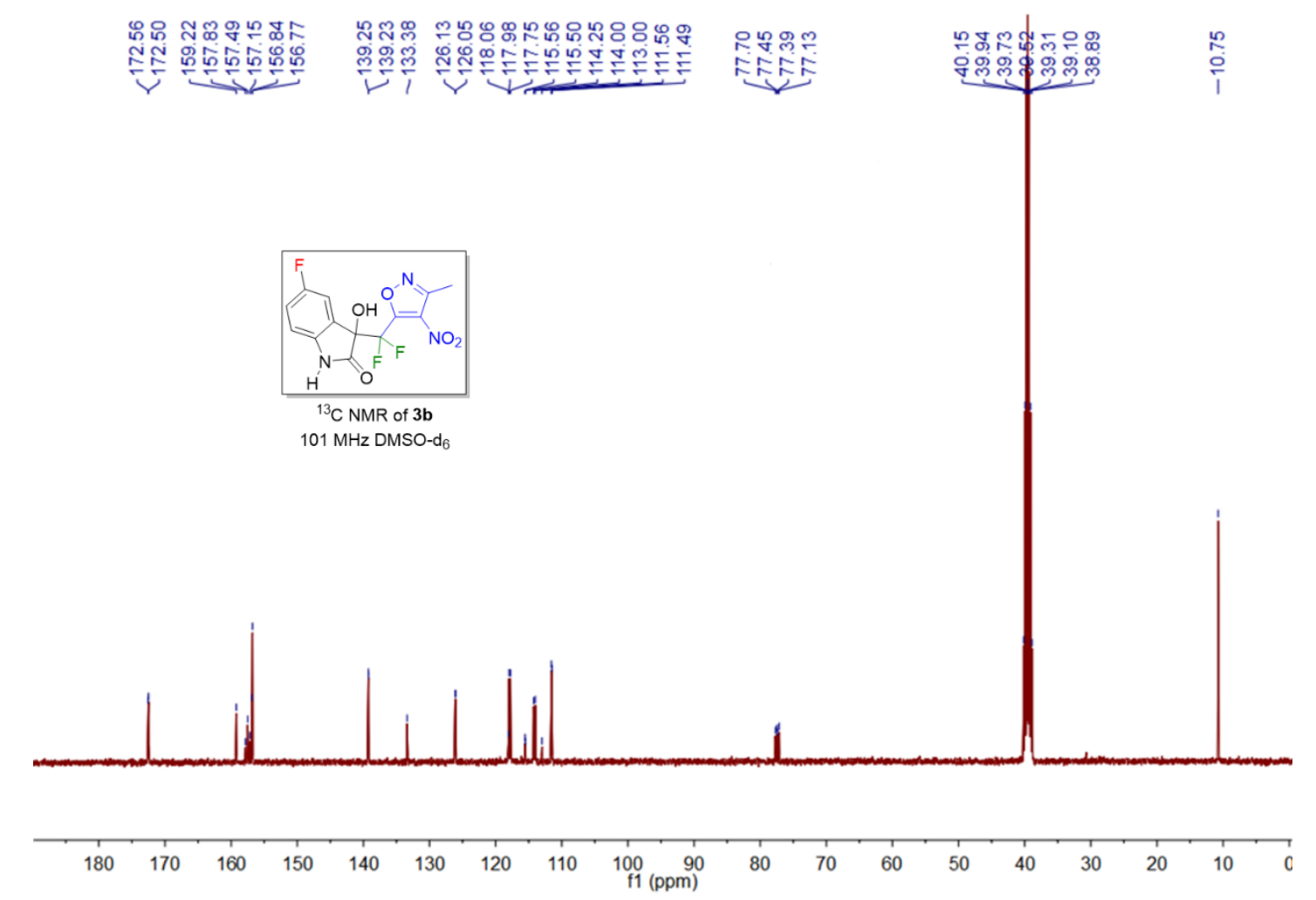

줒ํํ 중

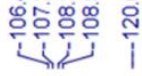
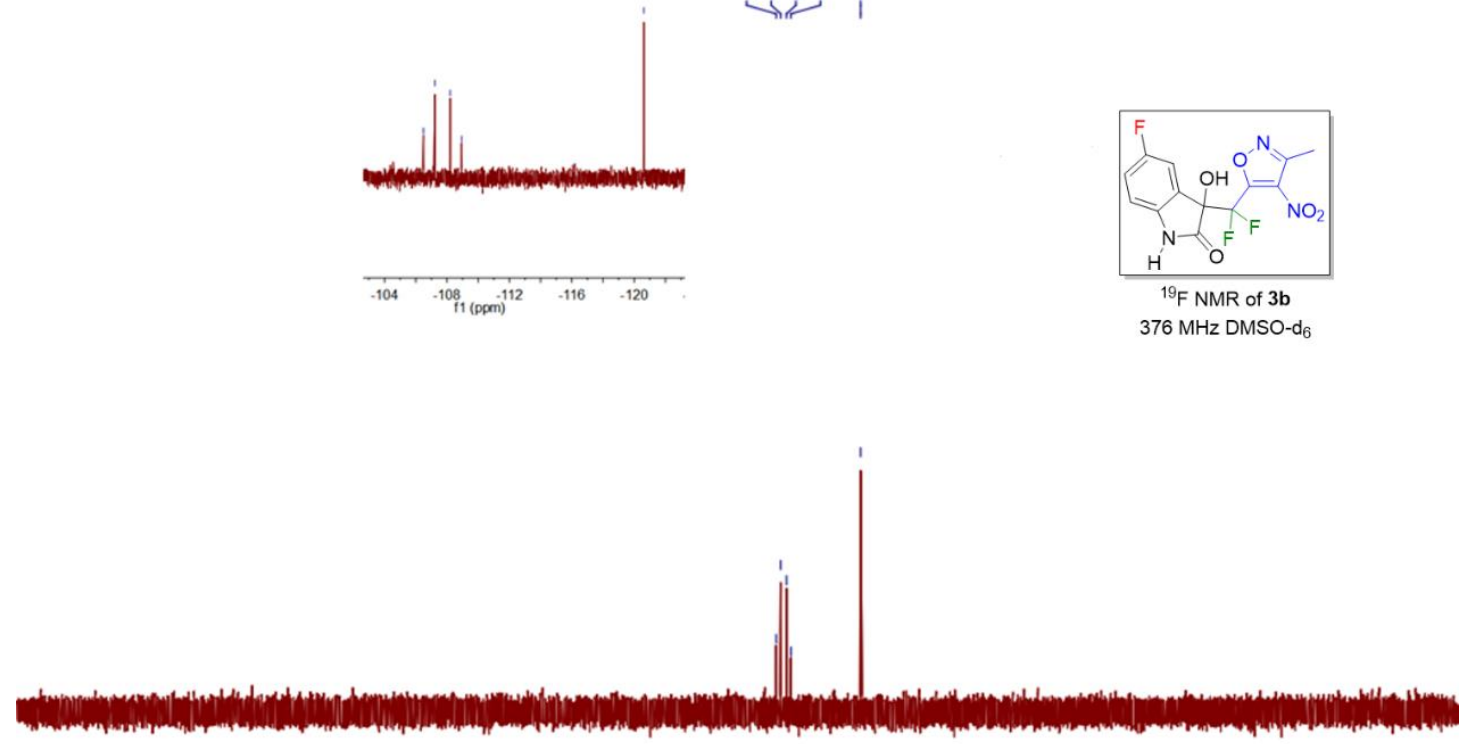

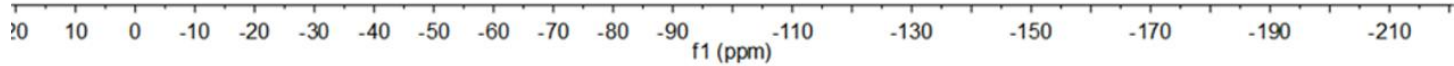




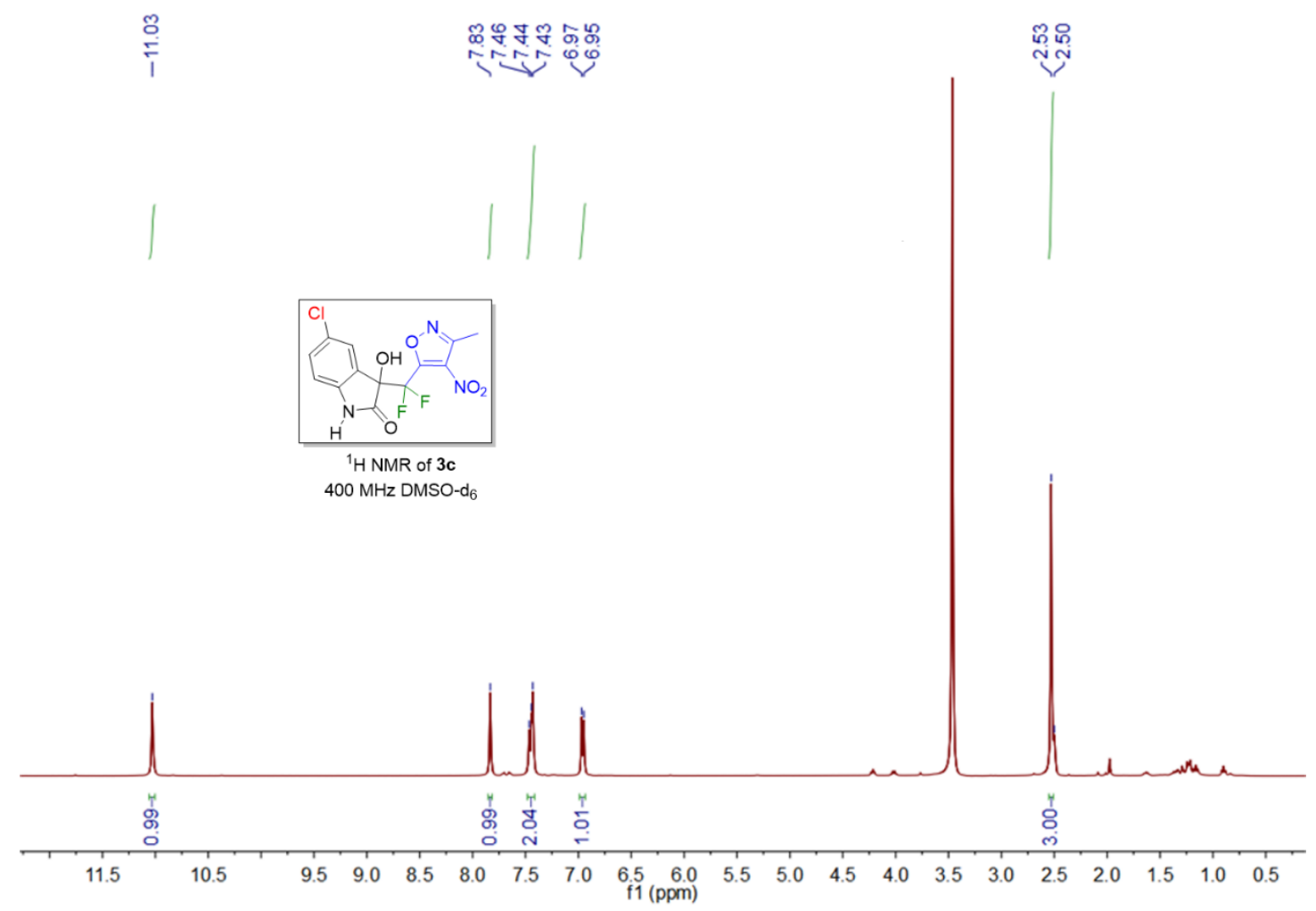

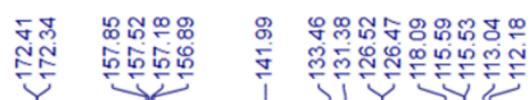

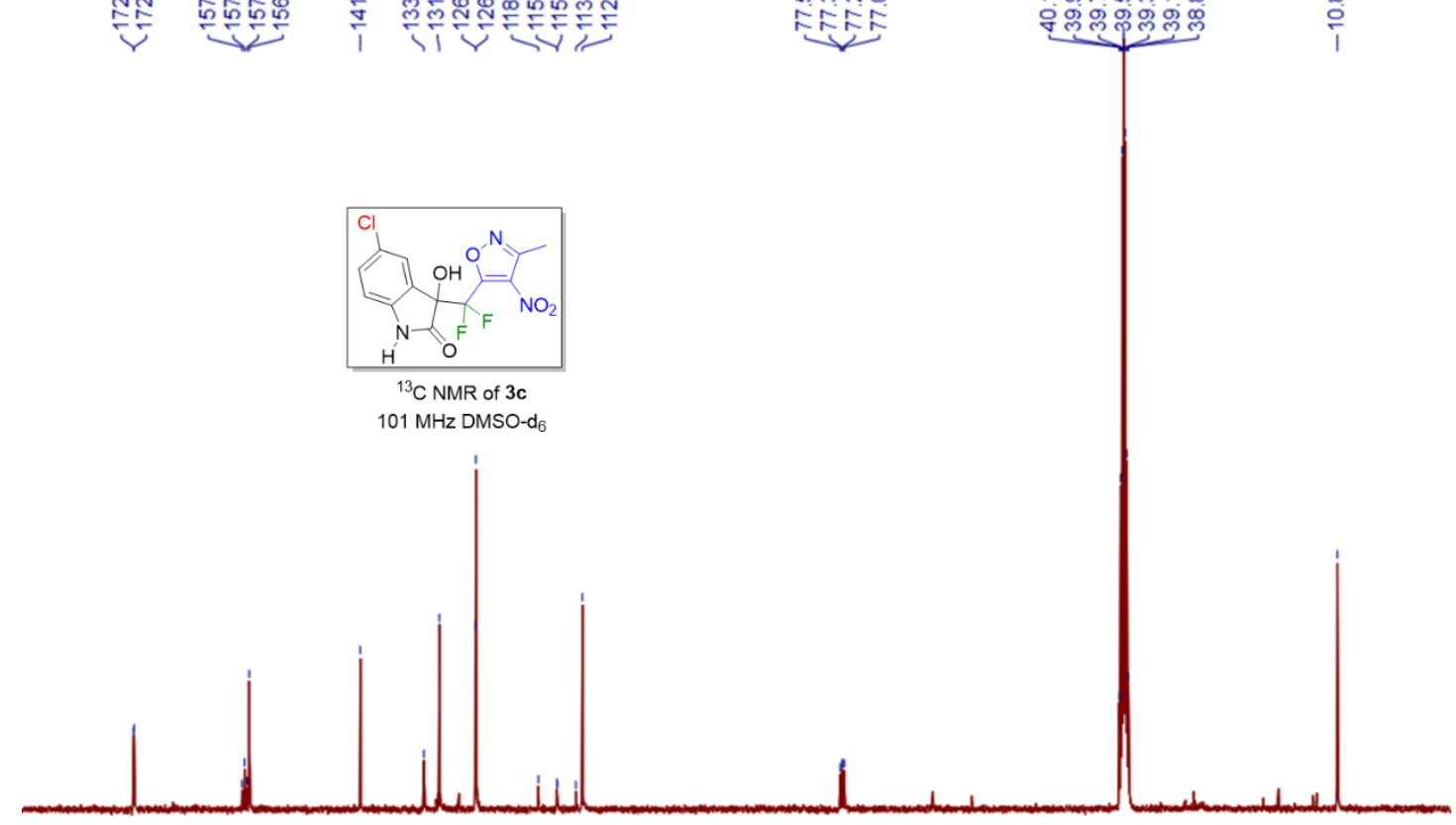

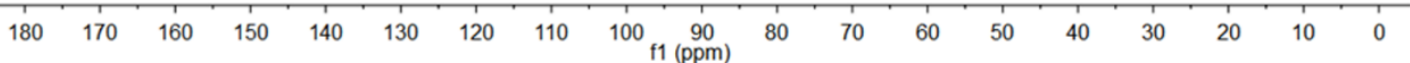



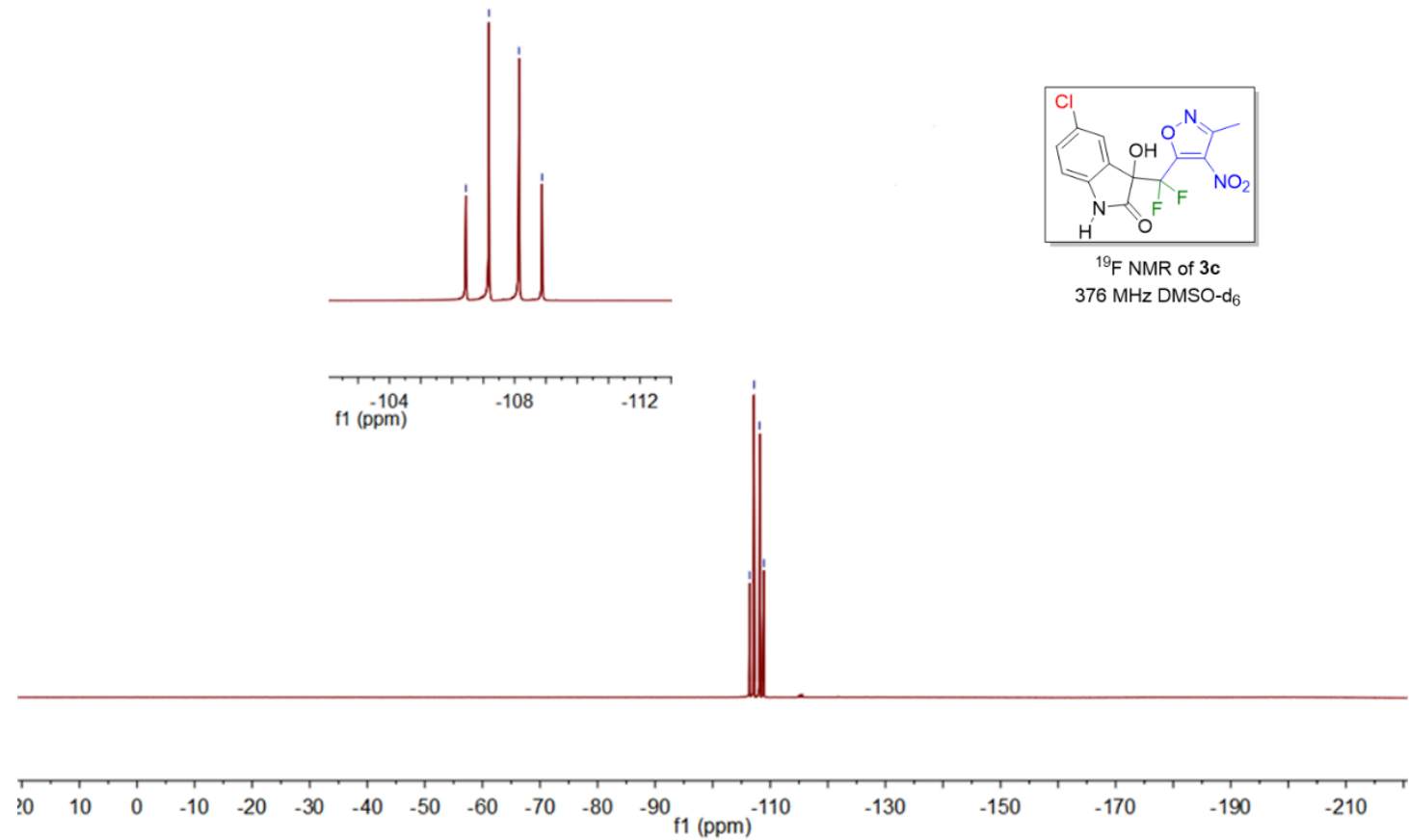

$376 \mathrm{MHz}$ DMSO-d
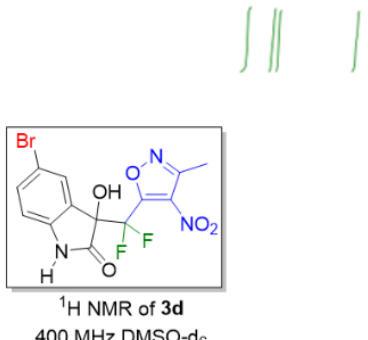

$400 \mathrm{MHz}$ DMSO-d

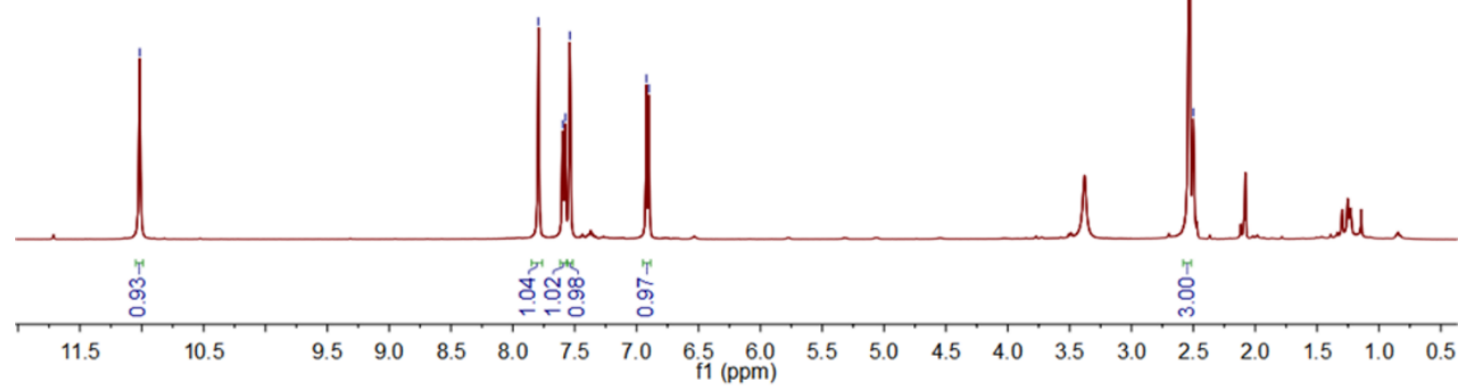



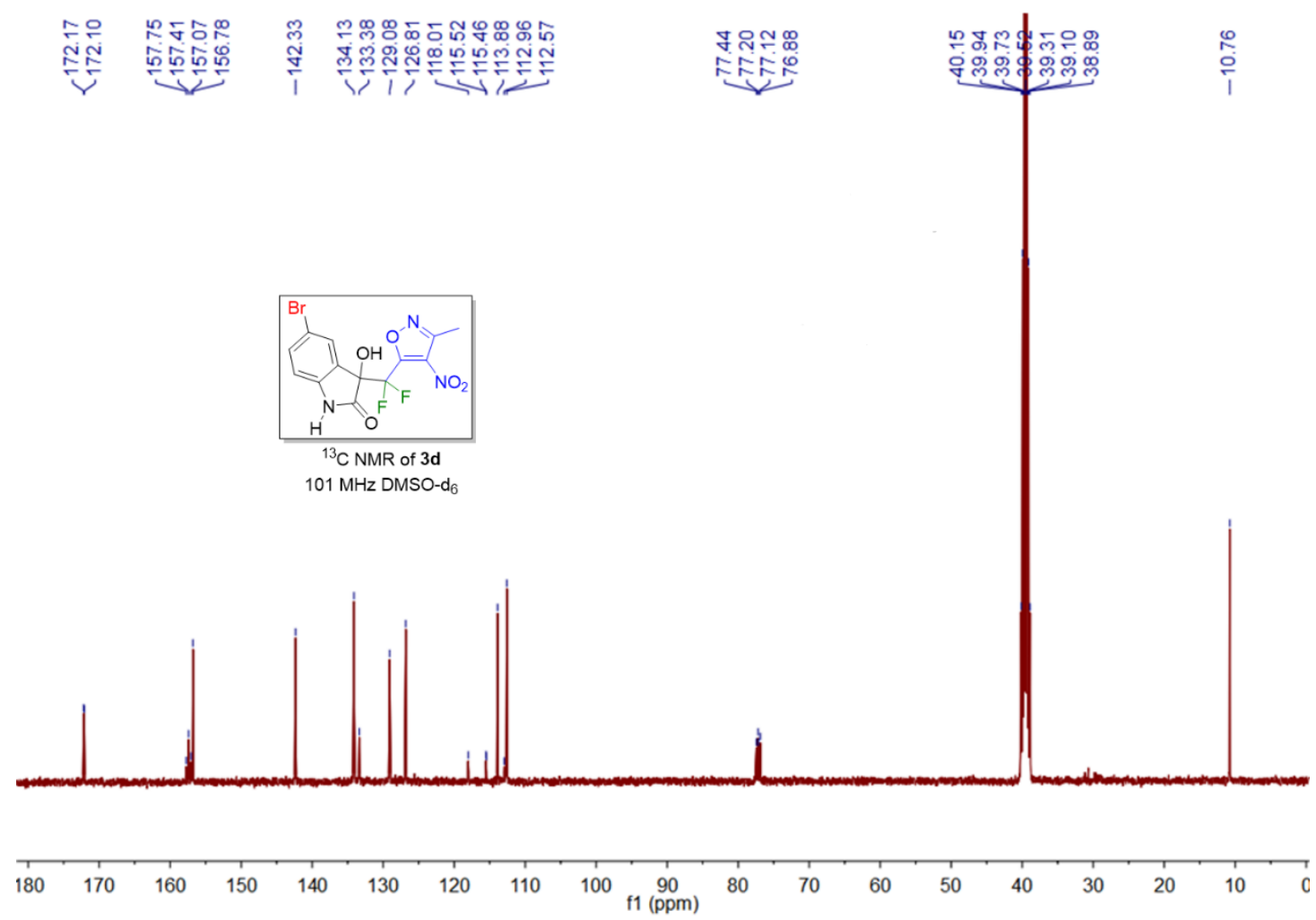

ํㅜ응

ํํㅇํํ

定
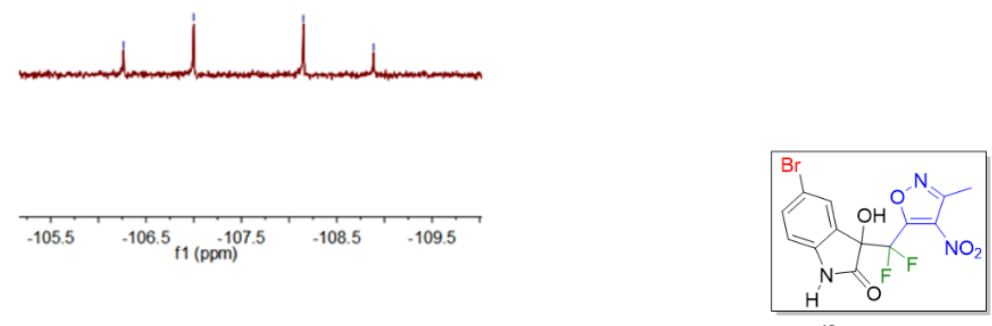

${ }^{19} \mathrm{~F}$ NMR of $\mathbf{3 d}$ $376 \mathrm{MHz}$ DMSO-d 6

\begin{tabular}{llllllllllllllllll}
\hline 0 & 10 & 0 & -10 & -20 & -30 & -40 & -50 & -60 & -70 & -80 & -90 & 1 & 1 & 1 & 1 & 1 & 1 \\
$\mathrm{f} 1(\mathrm{ppm})$ & -130 & -150 & -170 & -190 & -210
\end{tabular}



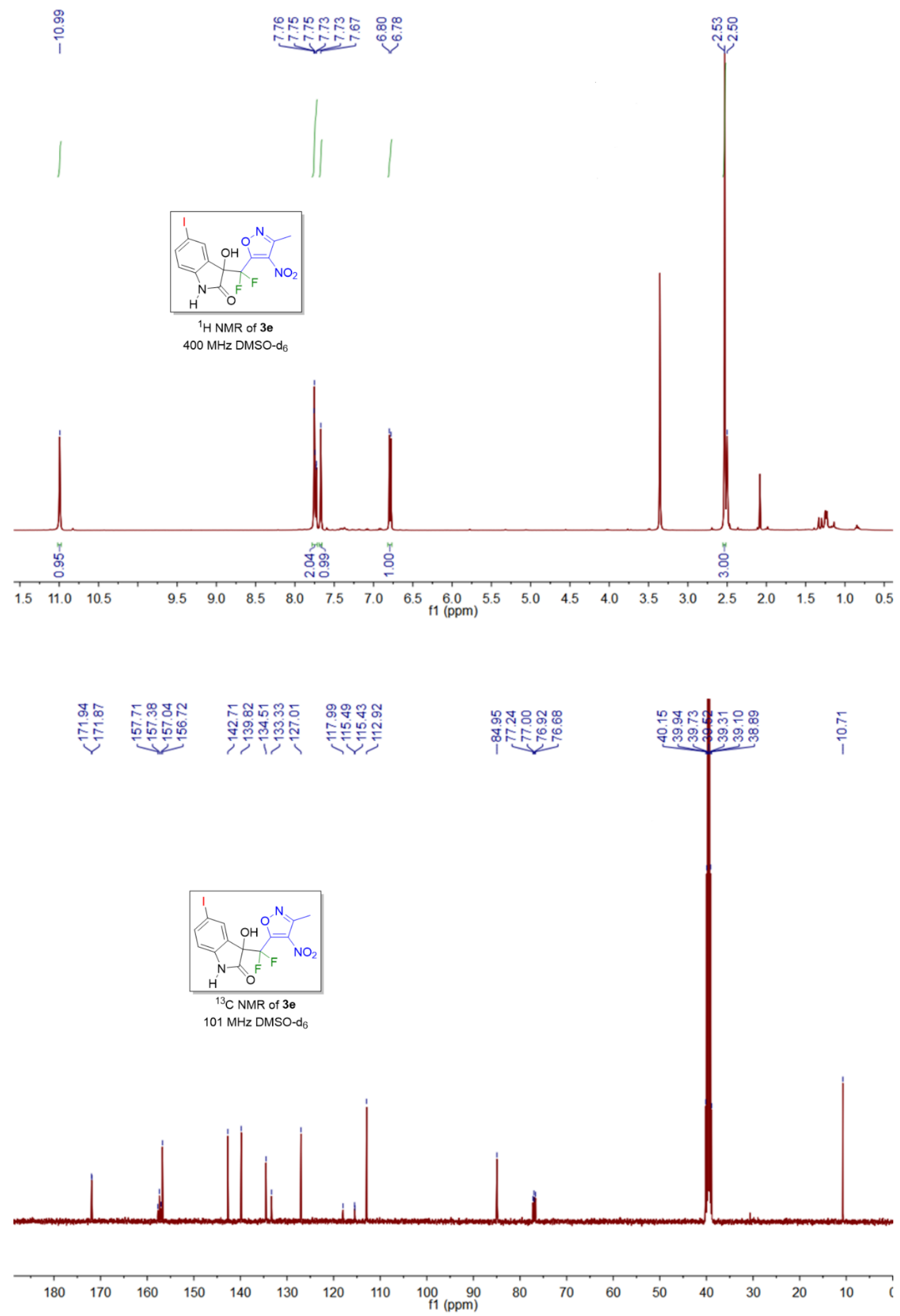
ำกำ

운운

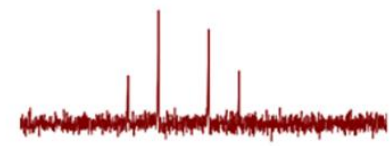

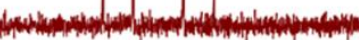

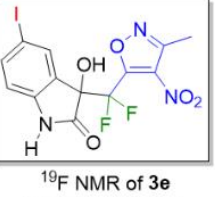

$376 \mathrm{MHz}$ DMSO-d

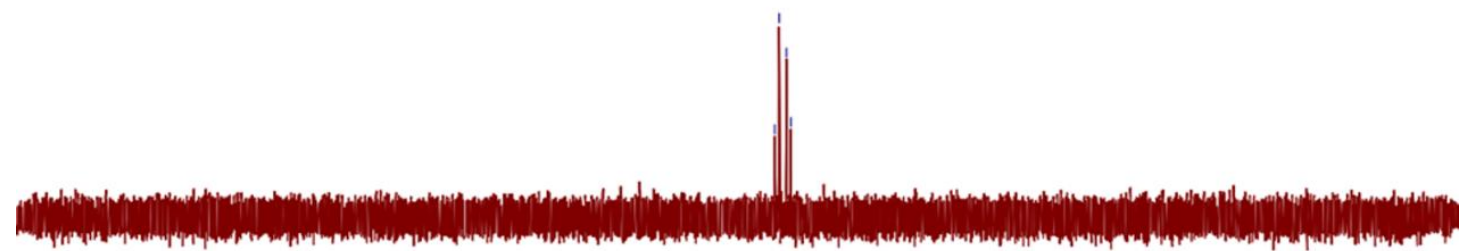

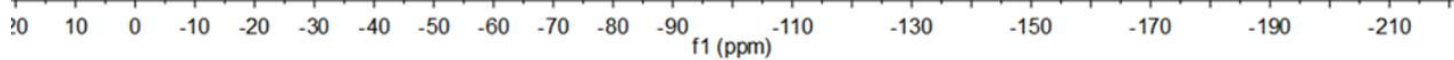

$\underbrace{\stackrel{0}{*}}_{1}$
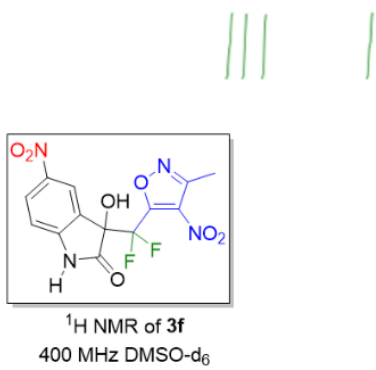

$400 \mathrm{MHz}$ DMSO-d

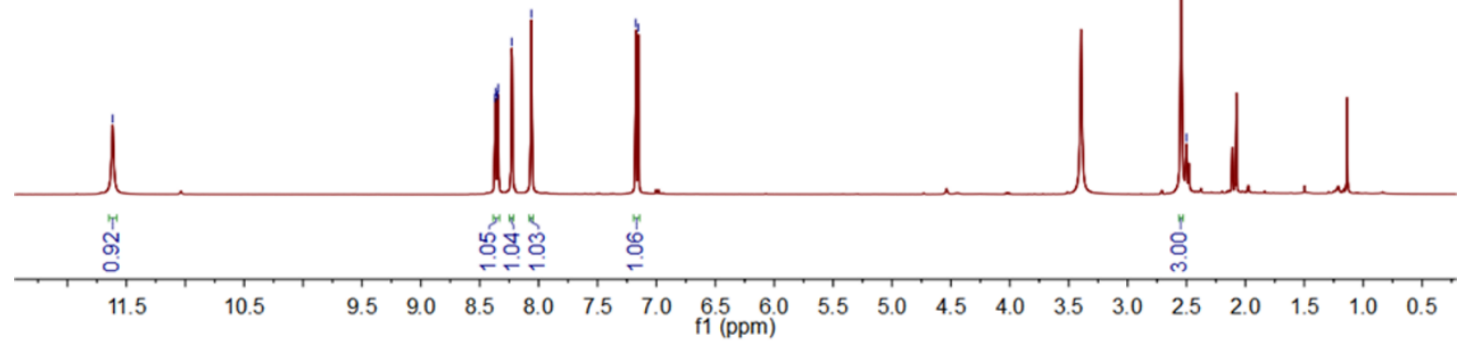



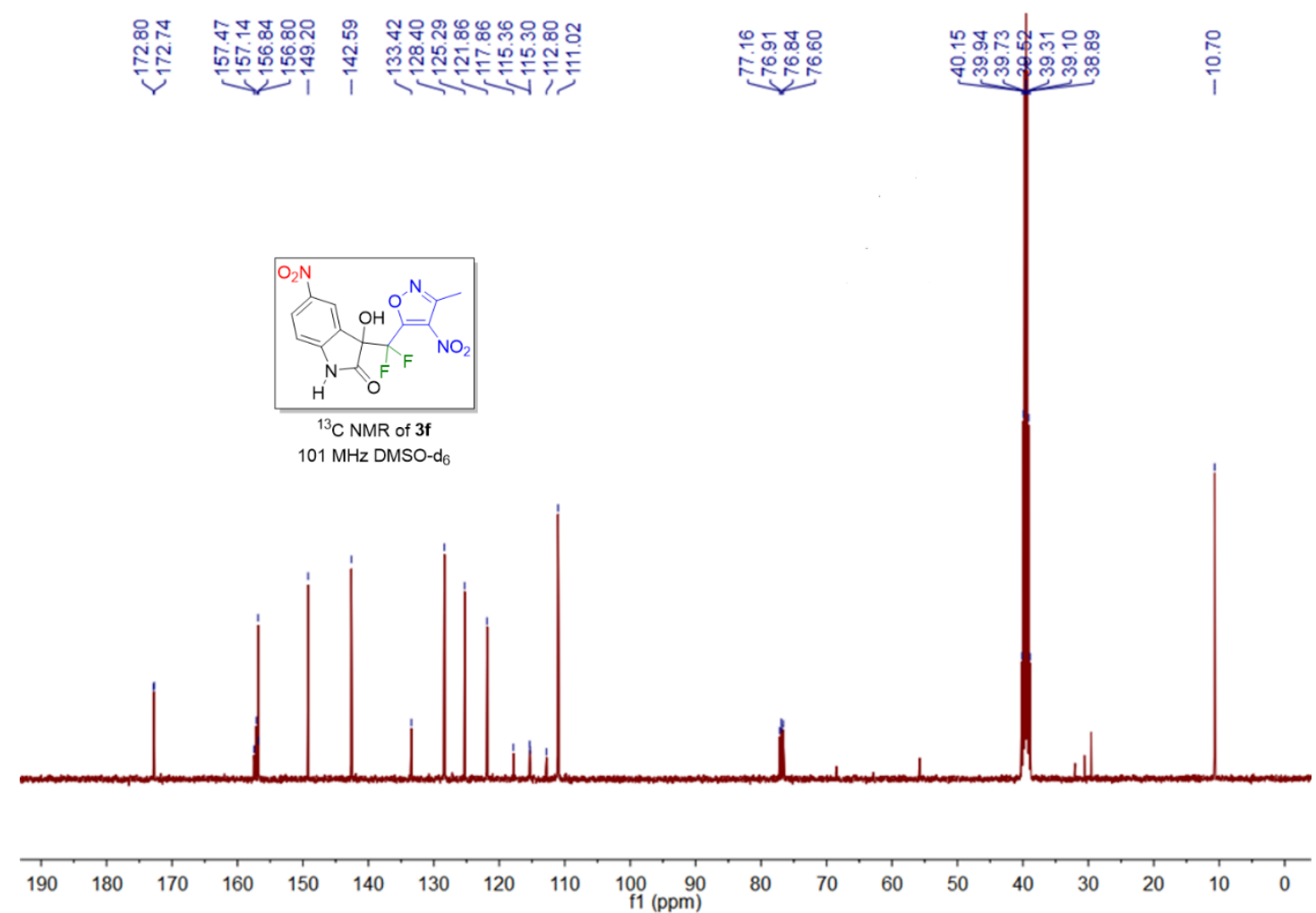

ำํํㅇ

응웅음

운욱

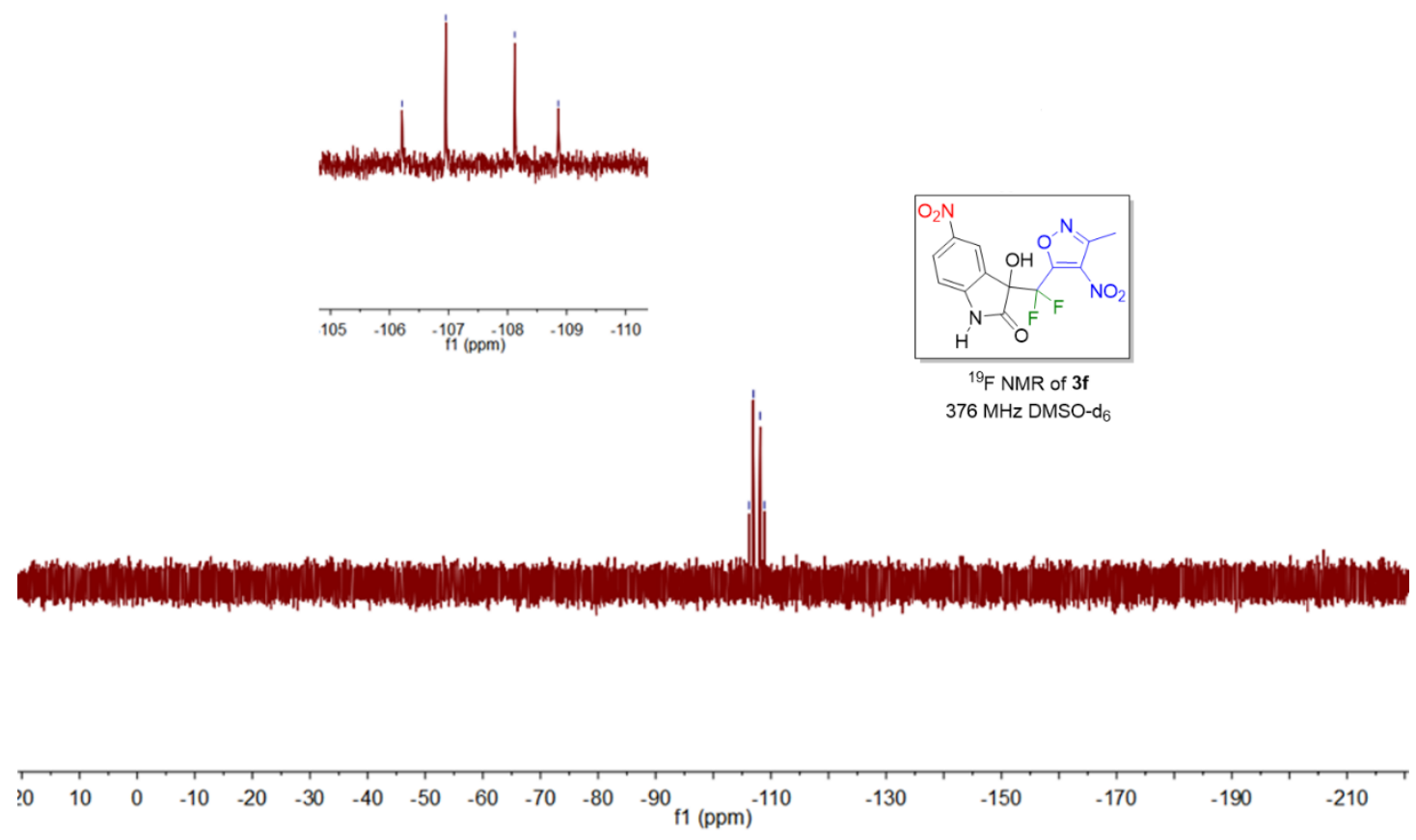




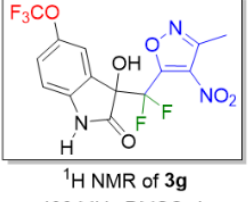

$400 \mathrm{MHz}$ DMSO-d

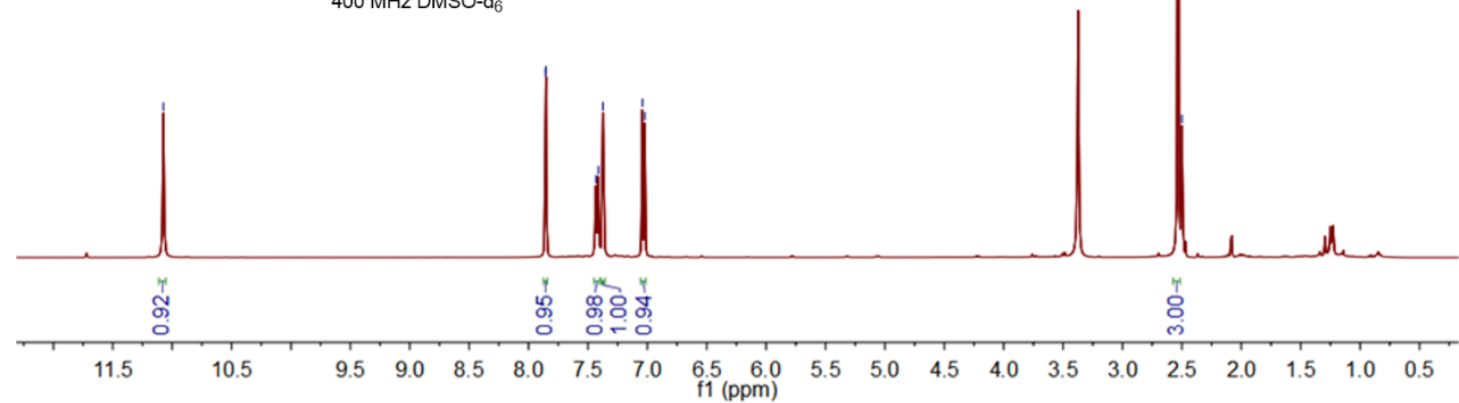

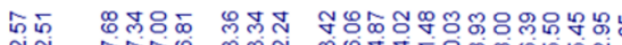

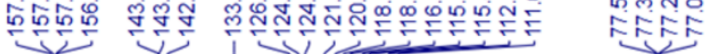

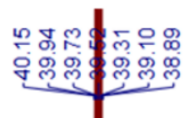

ำ

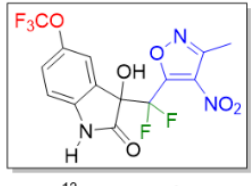

${ }^{13} \mathrm{C}$ NMR of $3 \mathrm{~g}$

$101 \mathrm{MHz}$ DMSO-d 6

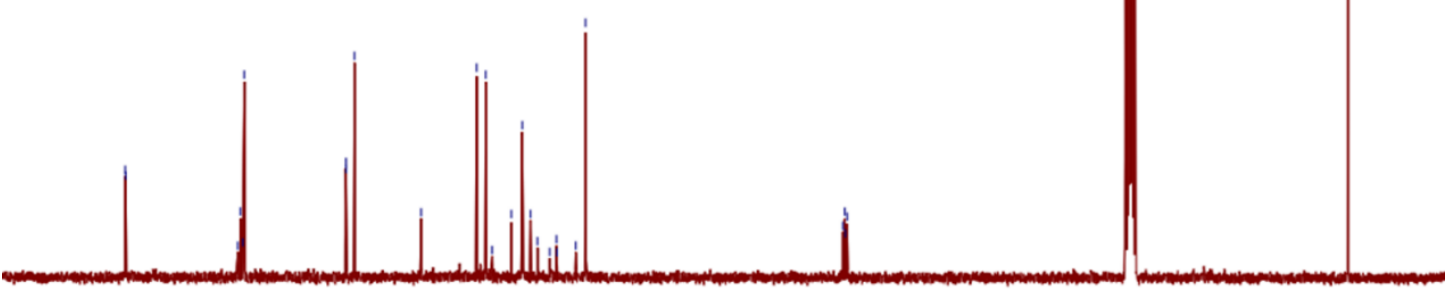

$\begin{array}{lllllllll}180 & 170 & 160 & 150 & 140 & 130 & 120 & 110 & 100 \begin{array}{l}90 \\ \mathrm{f} 1(\mathrm{ppm})\end{array}\end{array}$

$\begin{array}{lllllllll}80 & 70 & 60 & 50 & 40 & 30 & 20 & 10 & 0\end{array}$ 

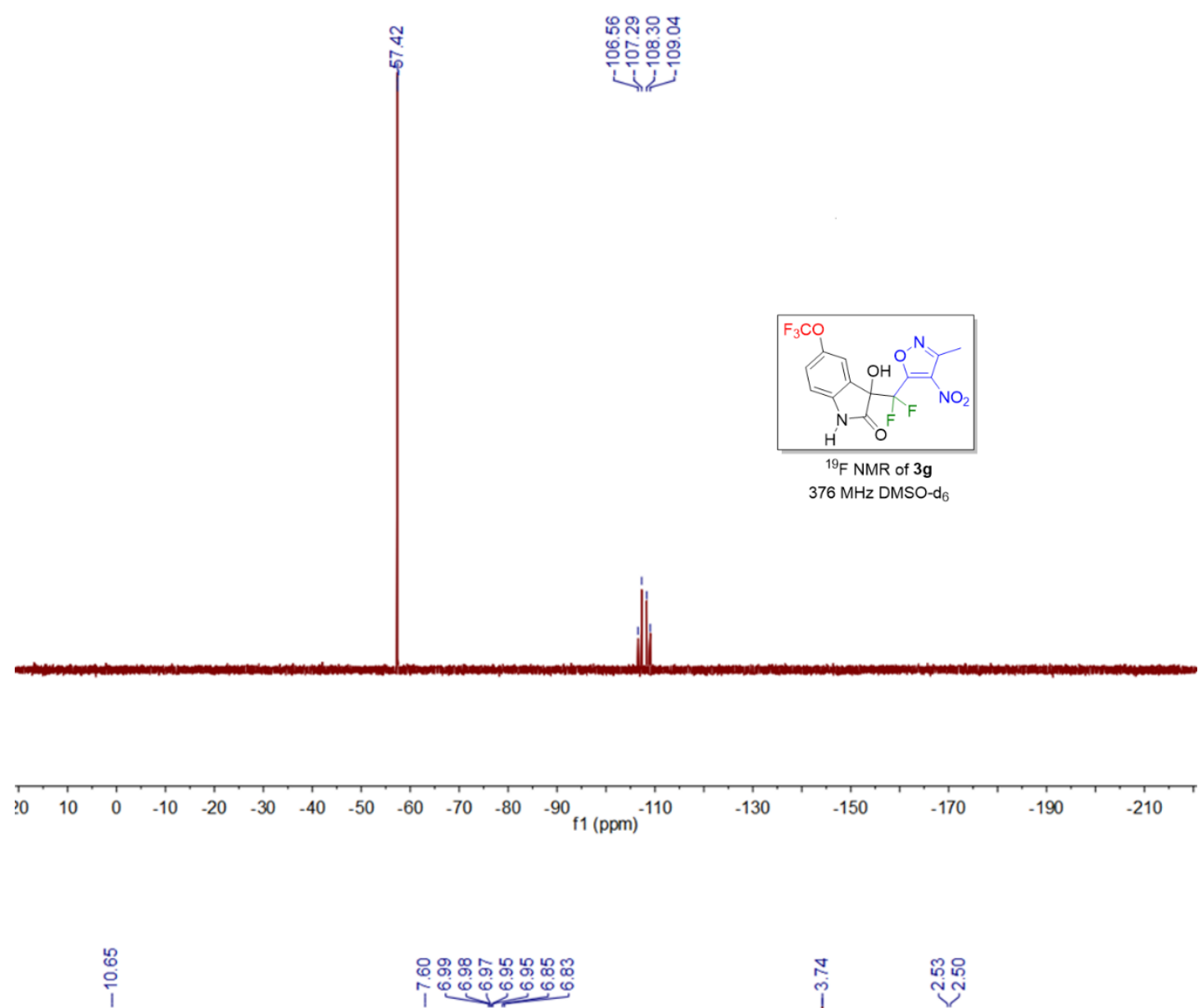

i
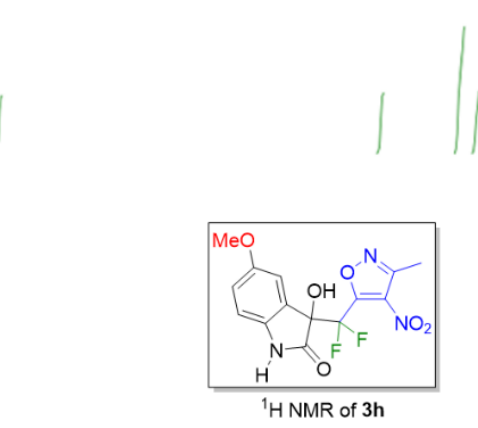

$400 \mathrm{MHz}$ DMSO-d 6

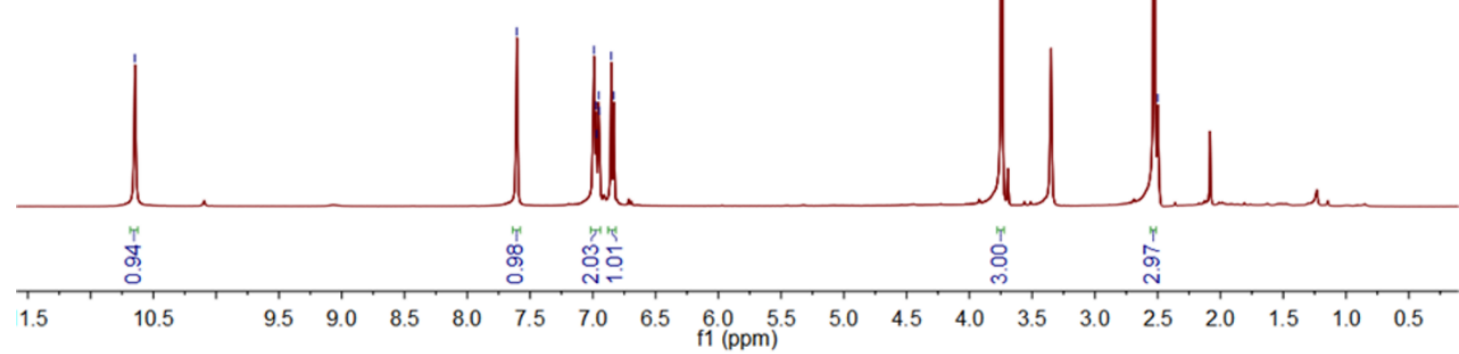



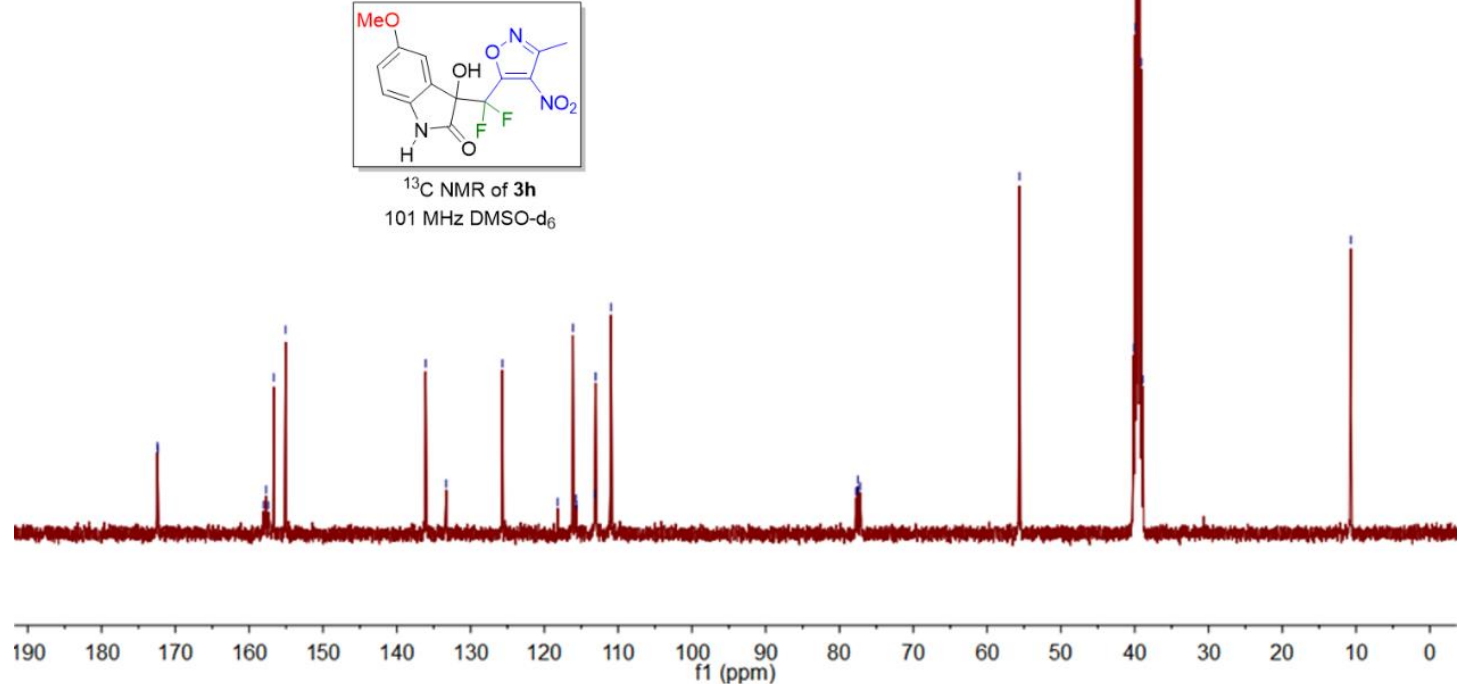

훙으응

连
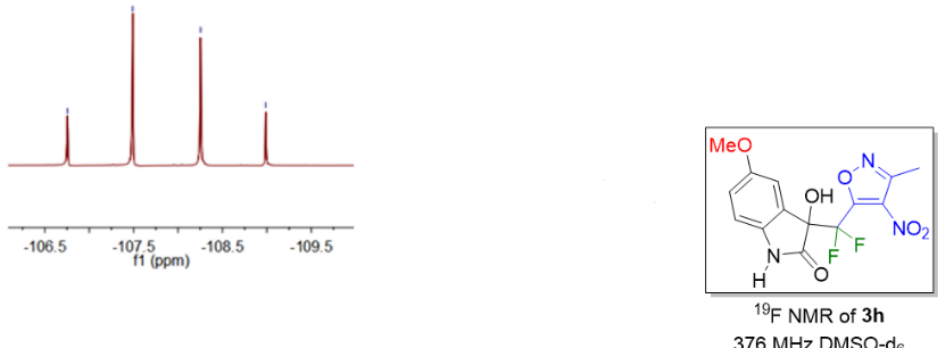

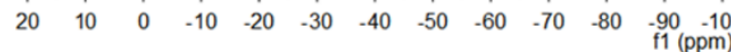

$-120 \quad-140$

$-160$

$-180 \quad-200$ 

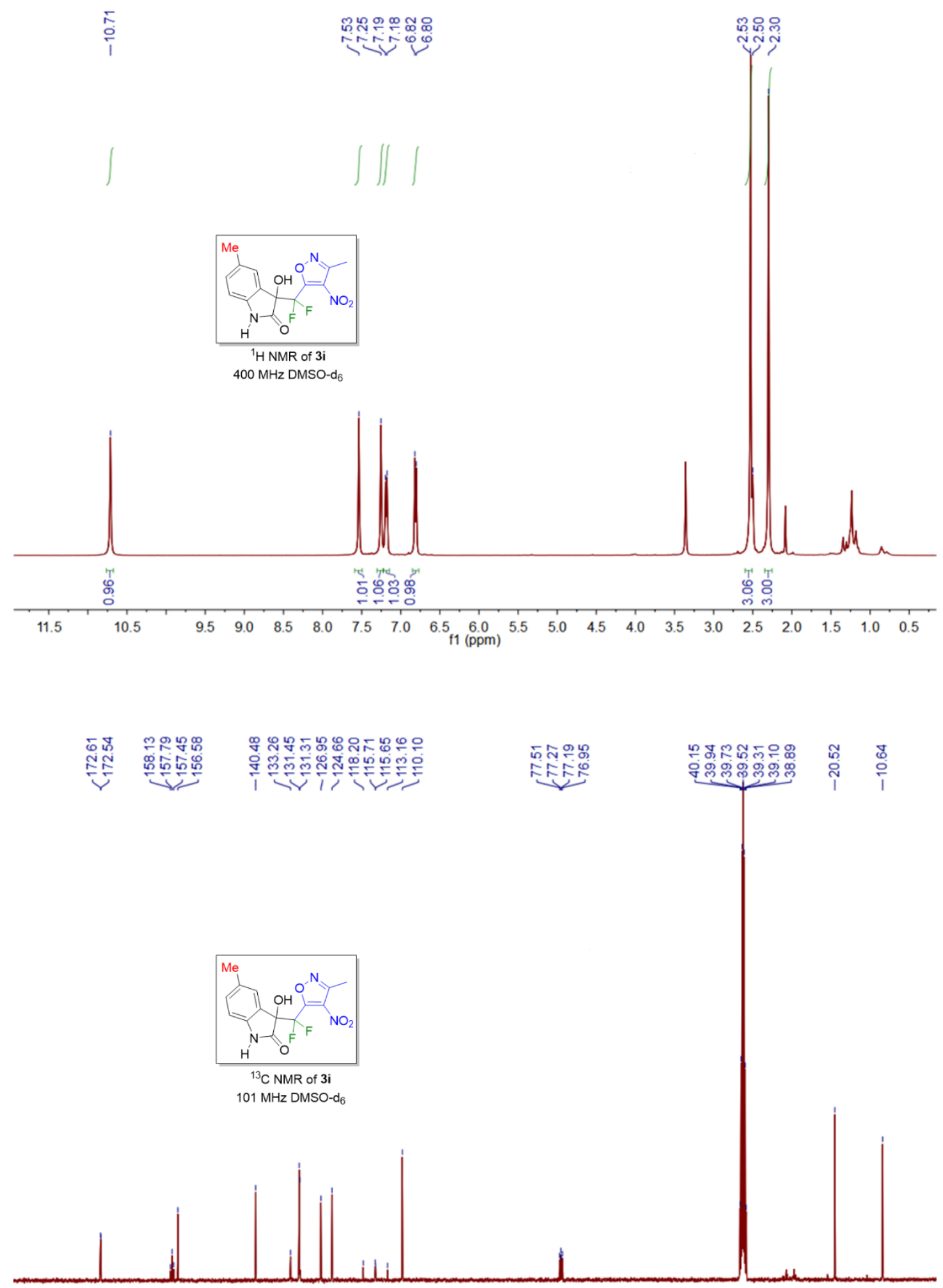

\begin{tabular}{llllllllllllllllllll}
\hline 1 & 180 & 170 & 160 & 150 & 140 & 130 & 120 & 110 & $\underset{\mathrm{f} 1}{100}(\mathrm{ppm})$ & 90 & 80 & 70 & 60 & 50 & 40 & 30 & 20 & 10 & 0
\end{tabular} 
ㄴากㅇำ

운윰ํำ
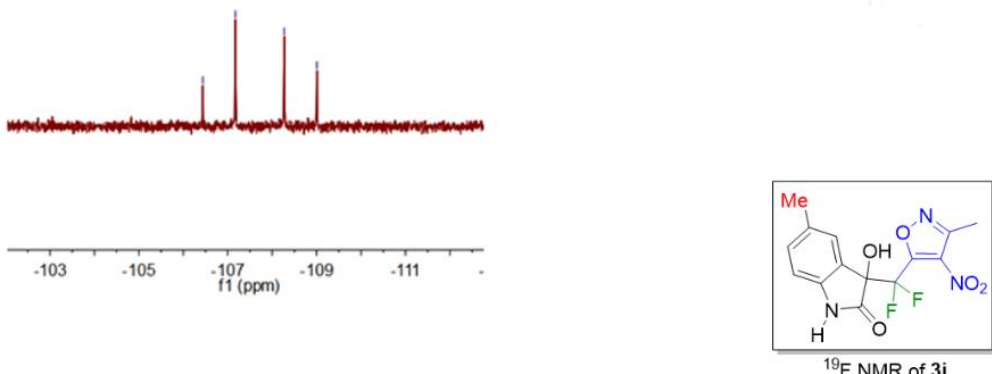

$376 \mathrm{MHz}$ DMSO-d
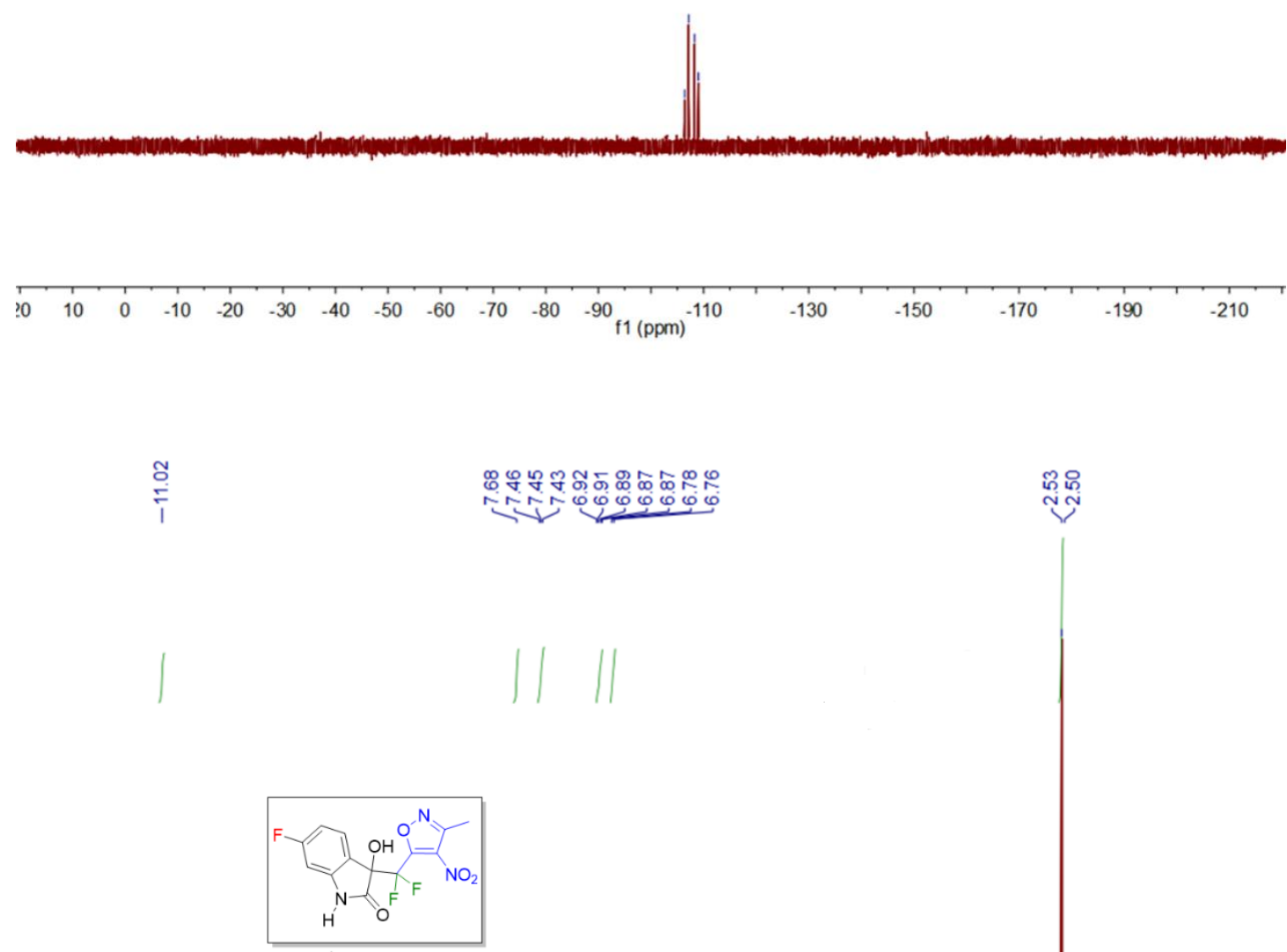

${ }^{1} \mathrm{H}$ NMR of $3 \mathrm{j}$

$400 \mathrm{MHz}$ DMSO-d 6

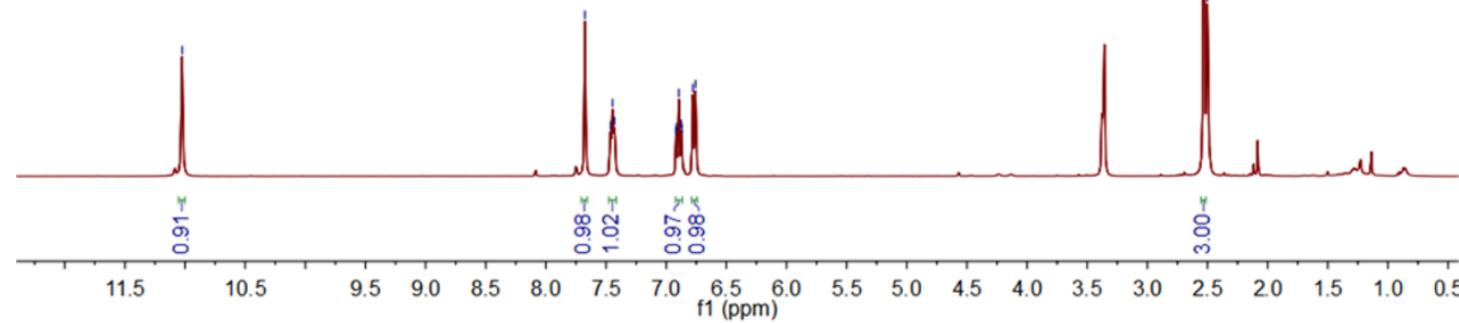




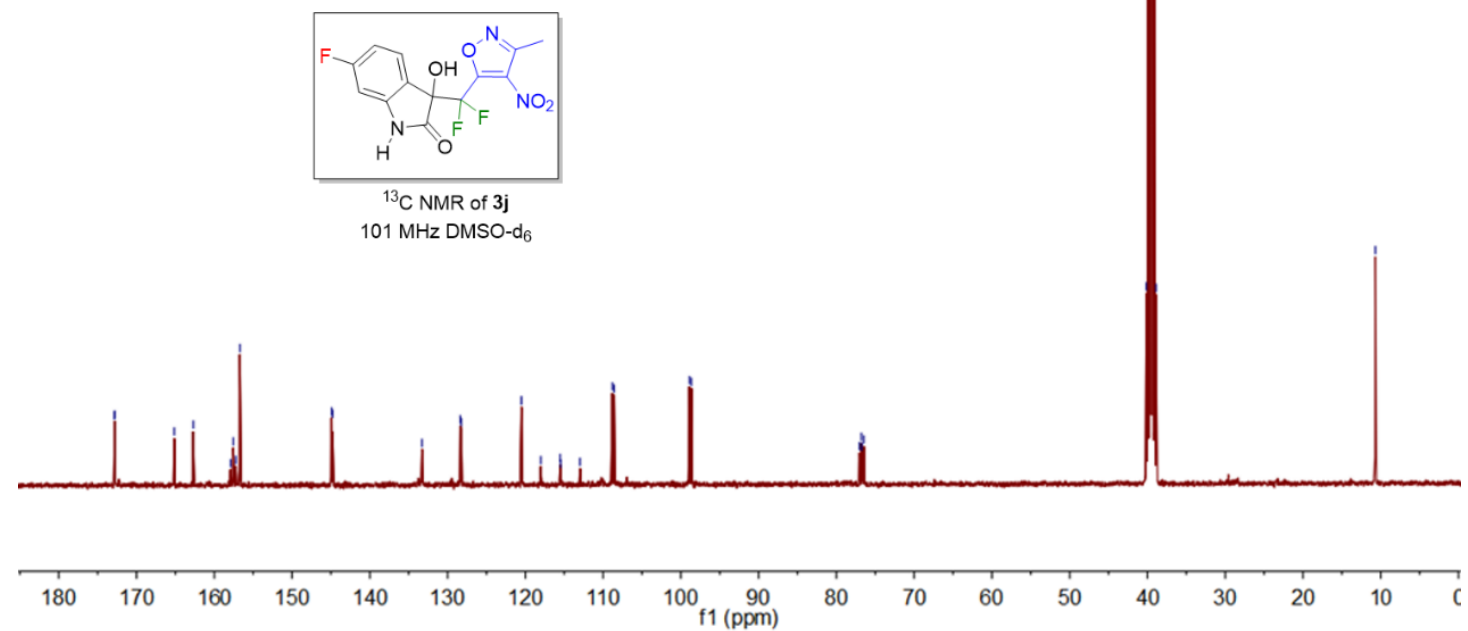

กู่

흥흐응응으

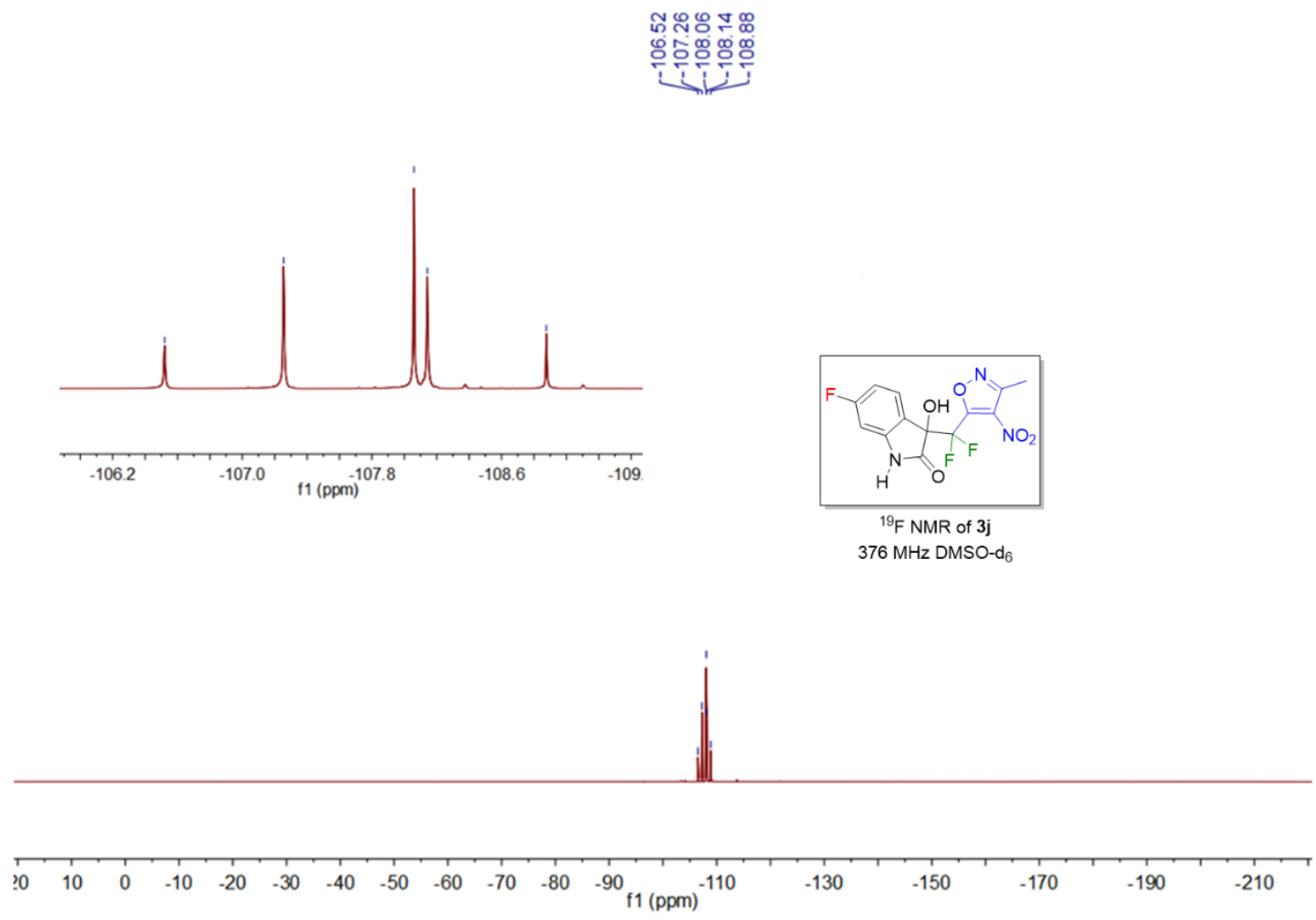



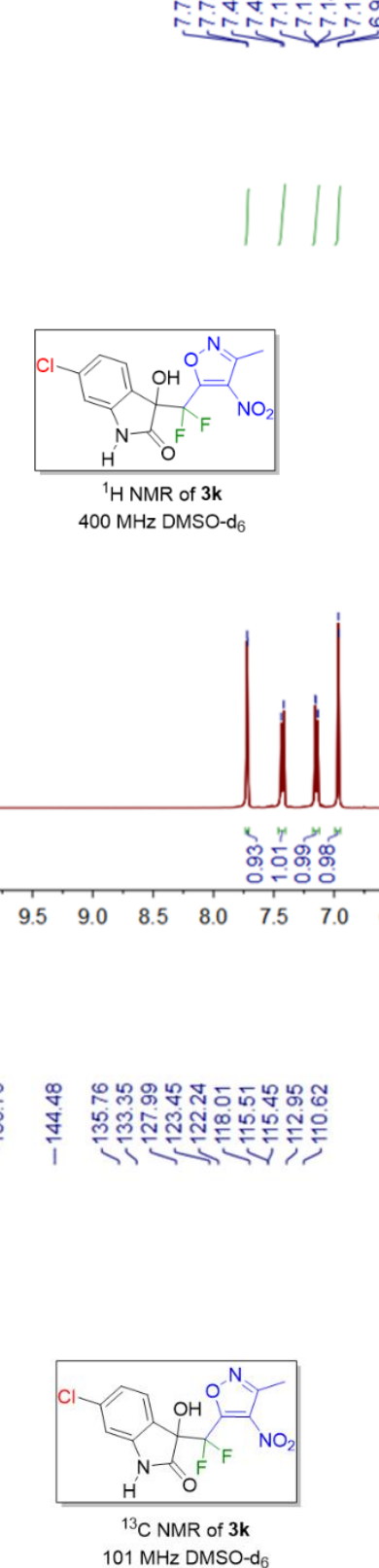
$101 \mathrm{MHz}$ DMSO-d

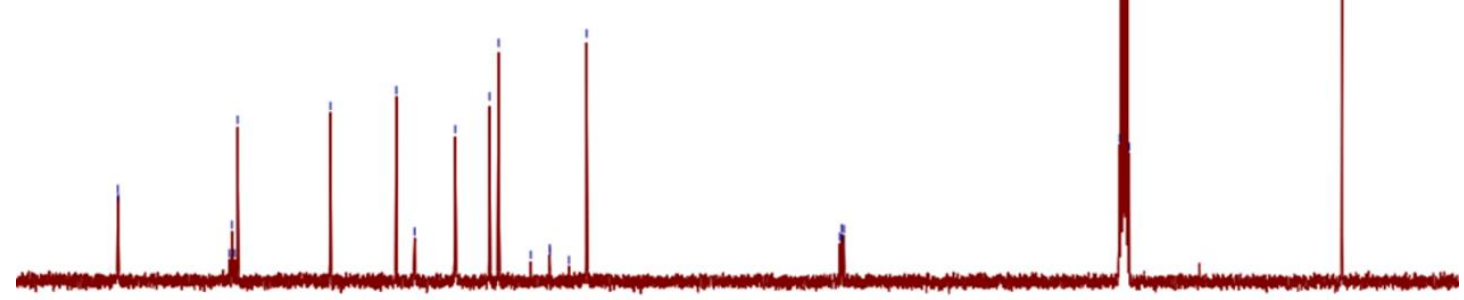

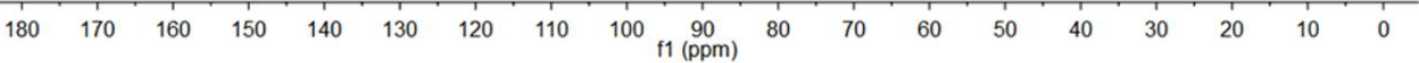



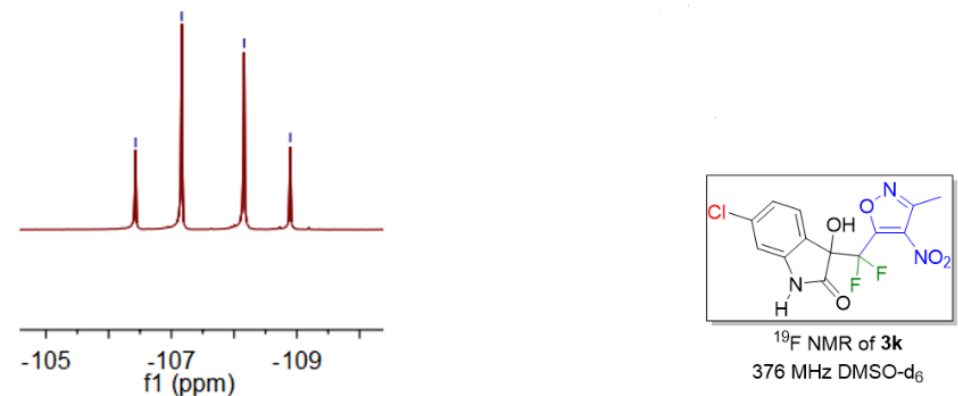

$376 \mathrm{MHz}$ DMSO-d 6

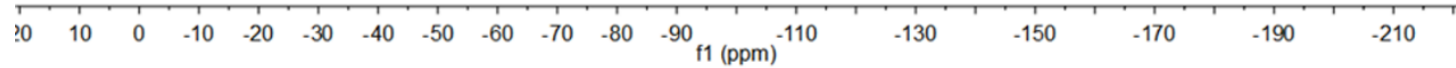

要
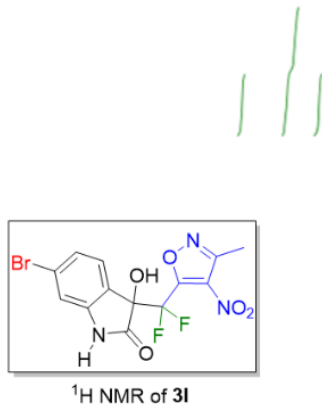

$400 \mathrm{MHz}$ DMSO-d

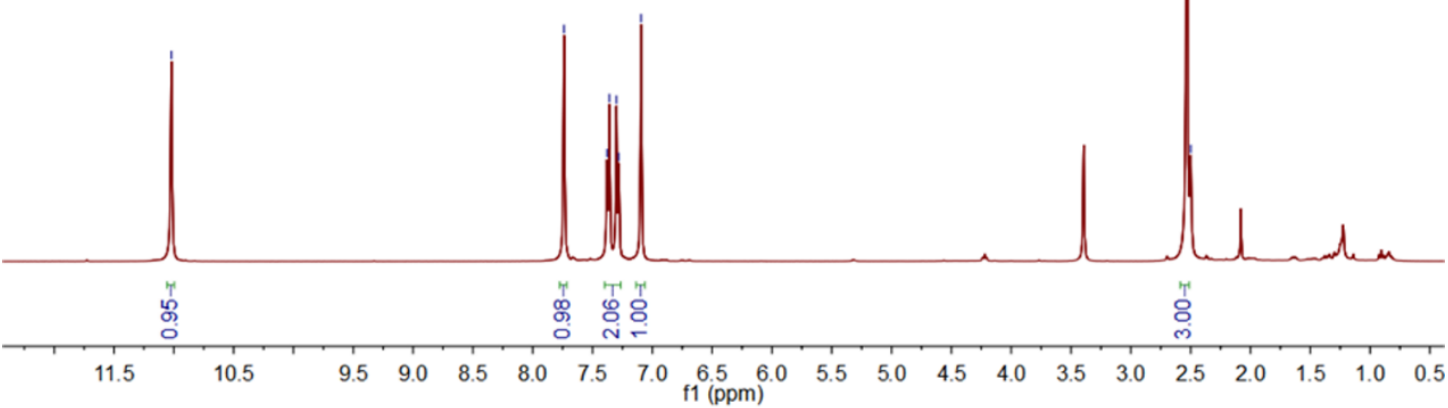




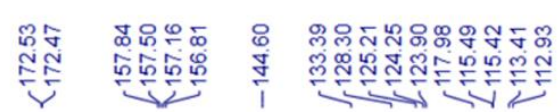

유ㅇㅛㅠ

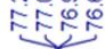

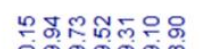

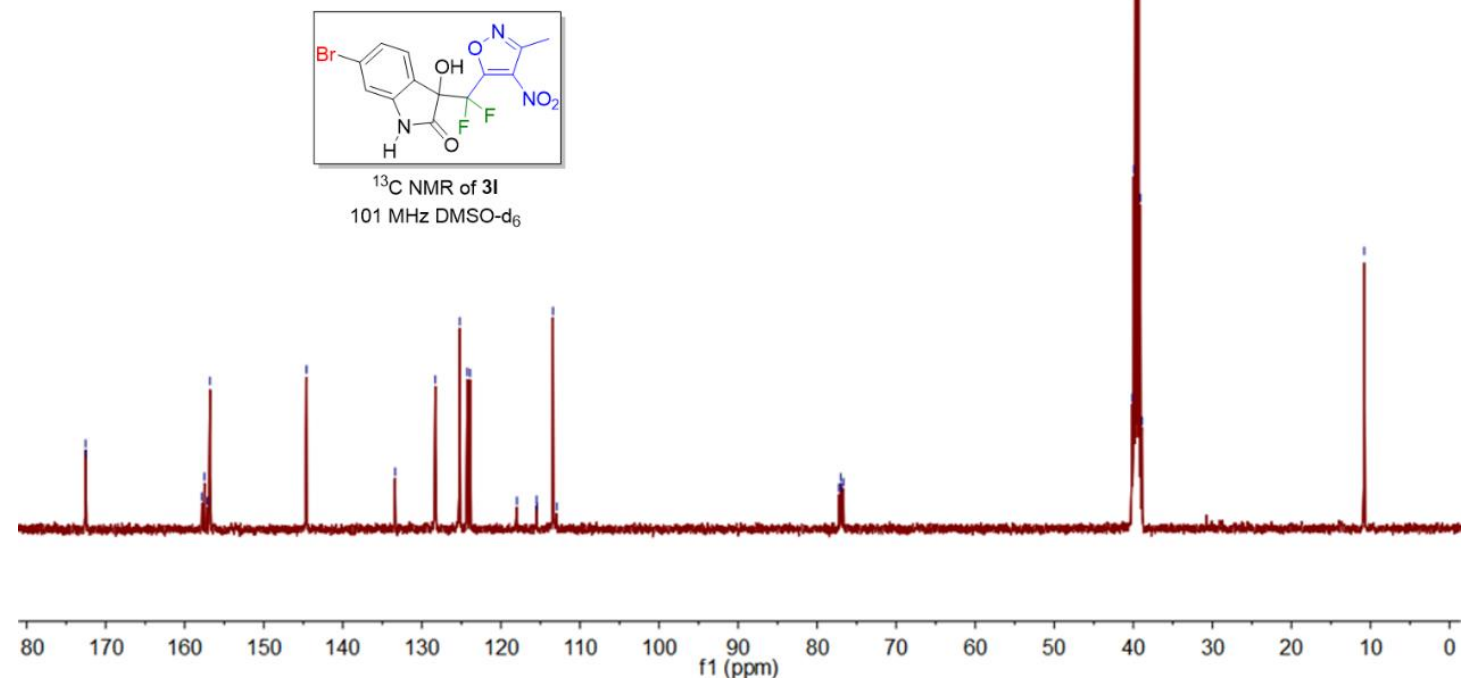

$101 \mathrm{MHz}$ DMSO-d 6

ํำ등

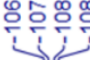
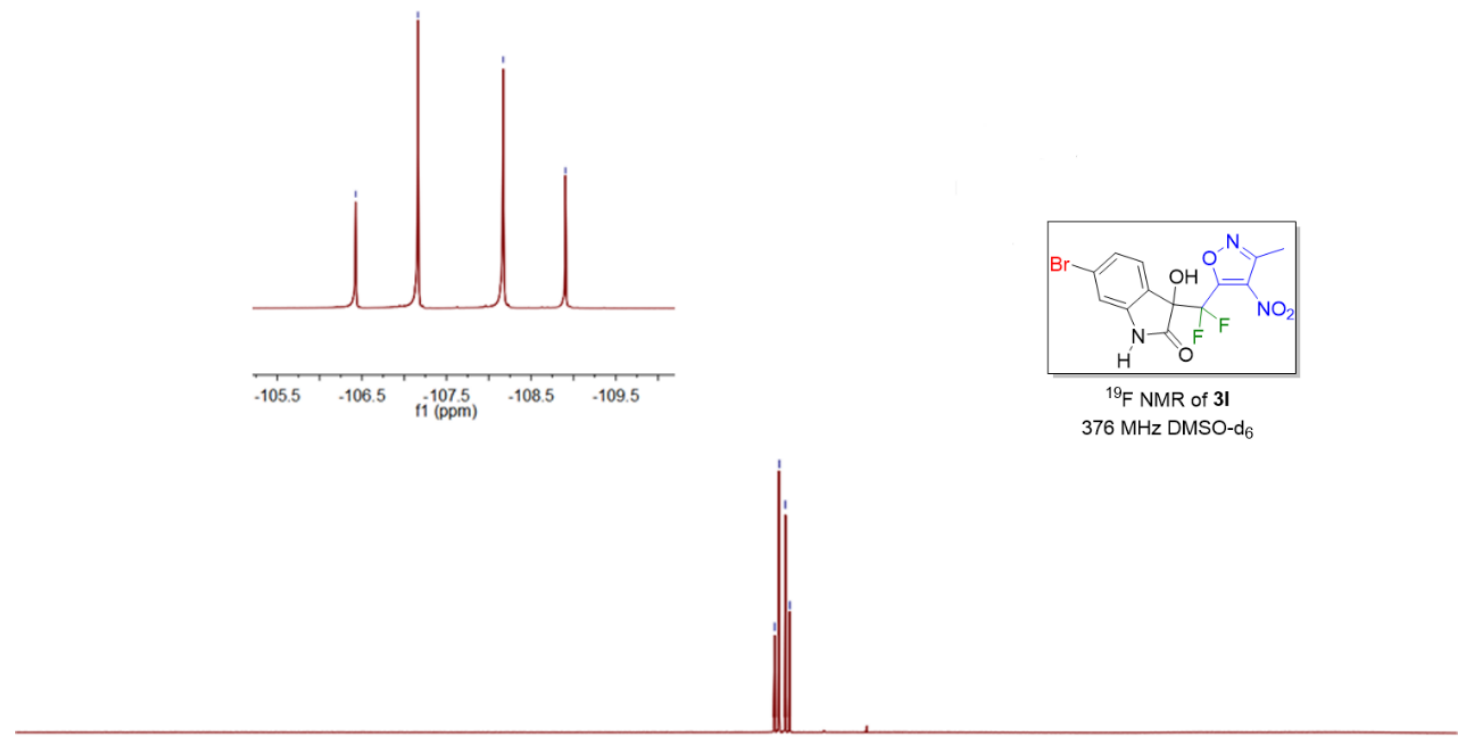

$\begin{array}{llllllllllllll}0 & 10 & 0 & -10 & -20 & -30 & -40 & -50 & -60 & -70 & -80 & -90 & & \\ \mathrm{f} 1(\mathrm{ppm}) & -11\end{array}$

$-130$

$-150$

$-190 \quad-210$ 

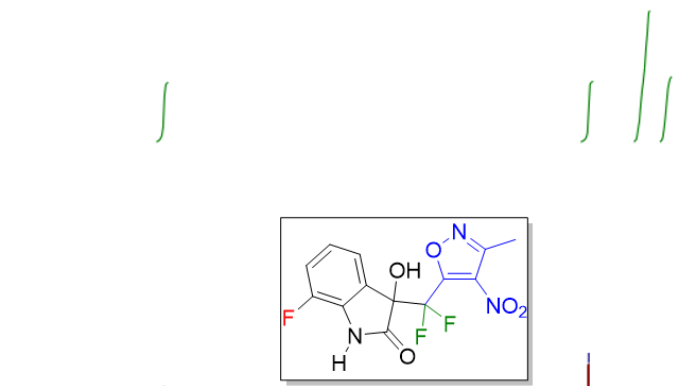

${ }^{1} \mathrm{H}$ NMR of $3 \mathrm{~m}$

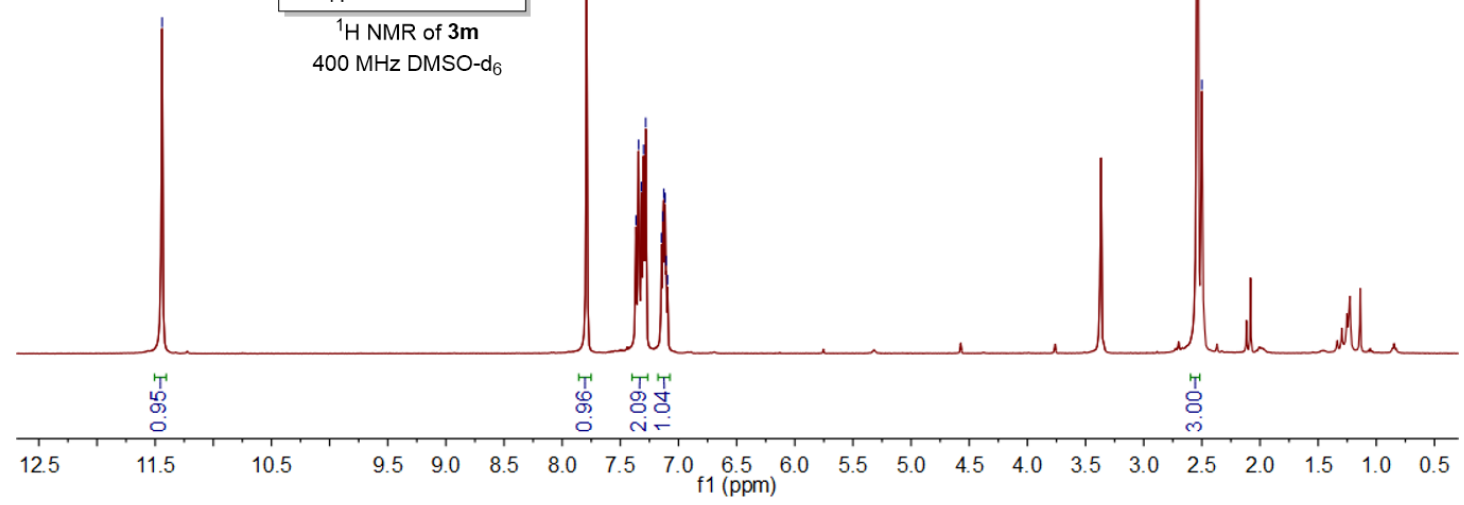

กู้

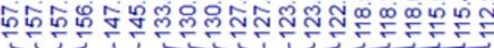

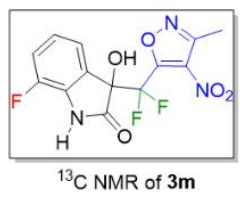

$101 \mathrm{MHz}$ DMSO-d

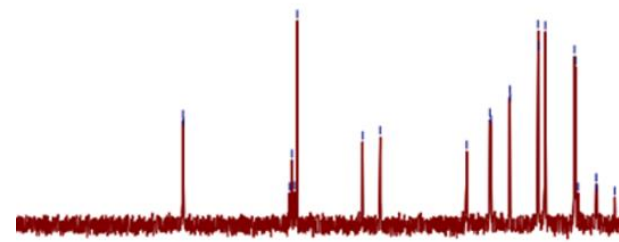




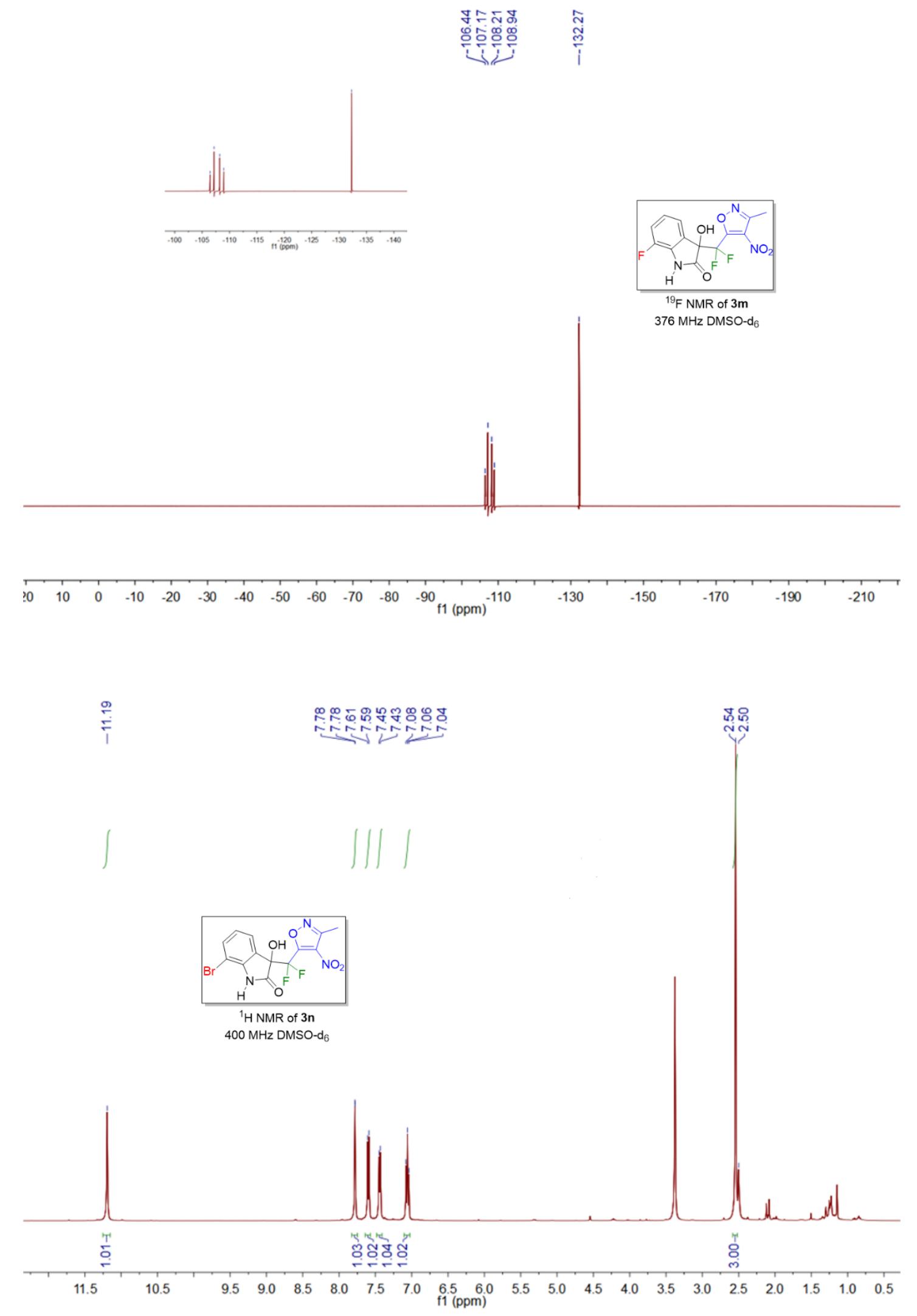



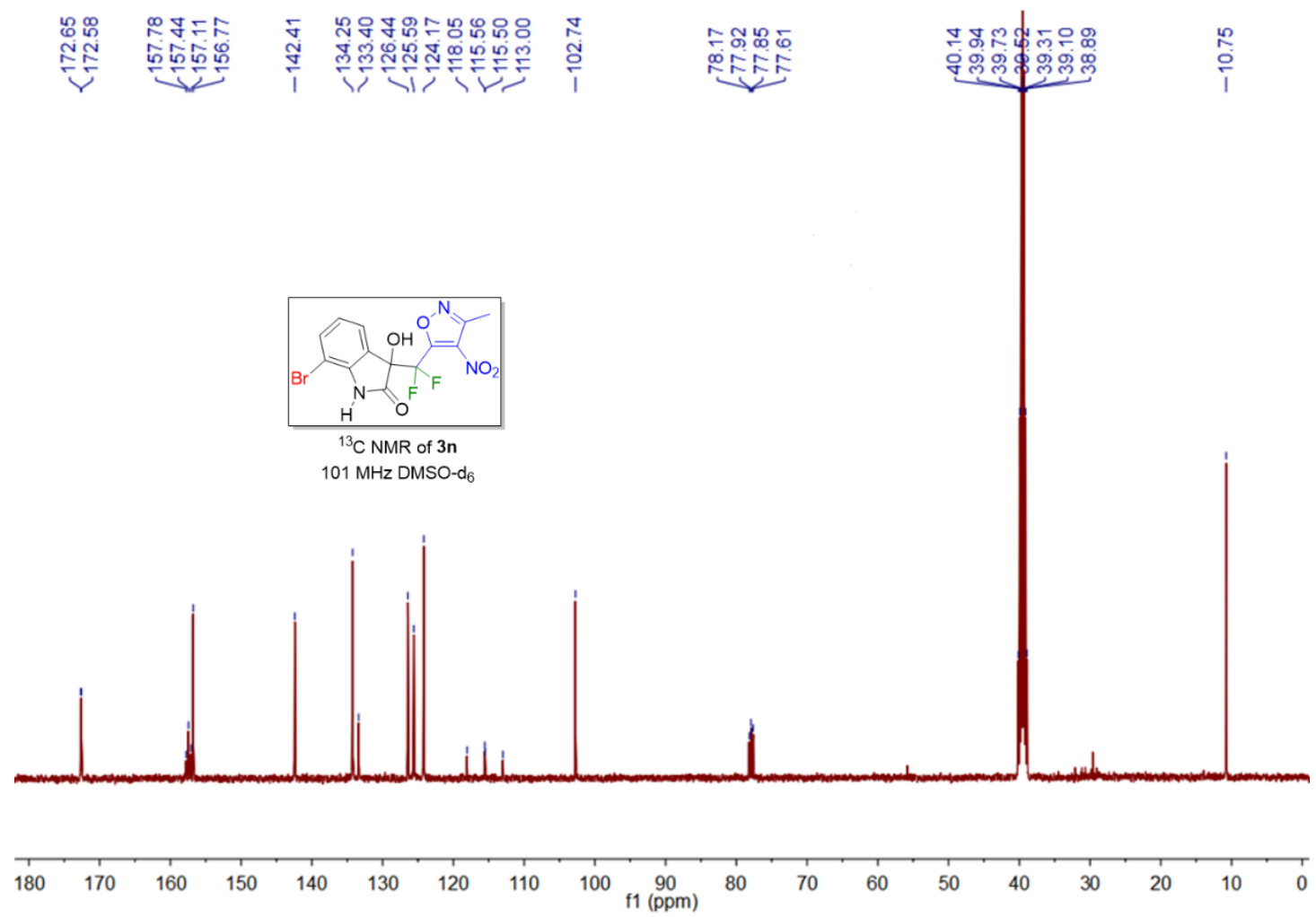

๓ำ듬

ㅇํㅇㅇํ음

운윽
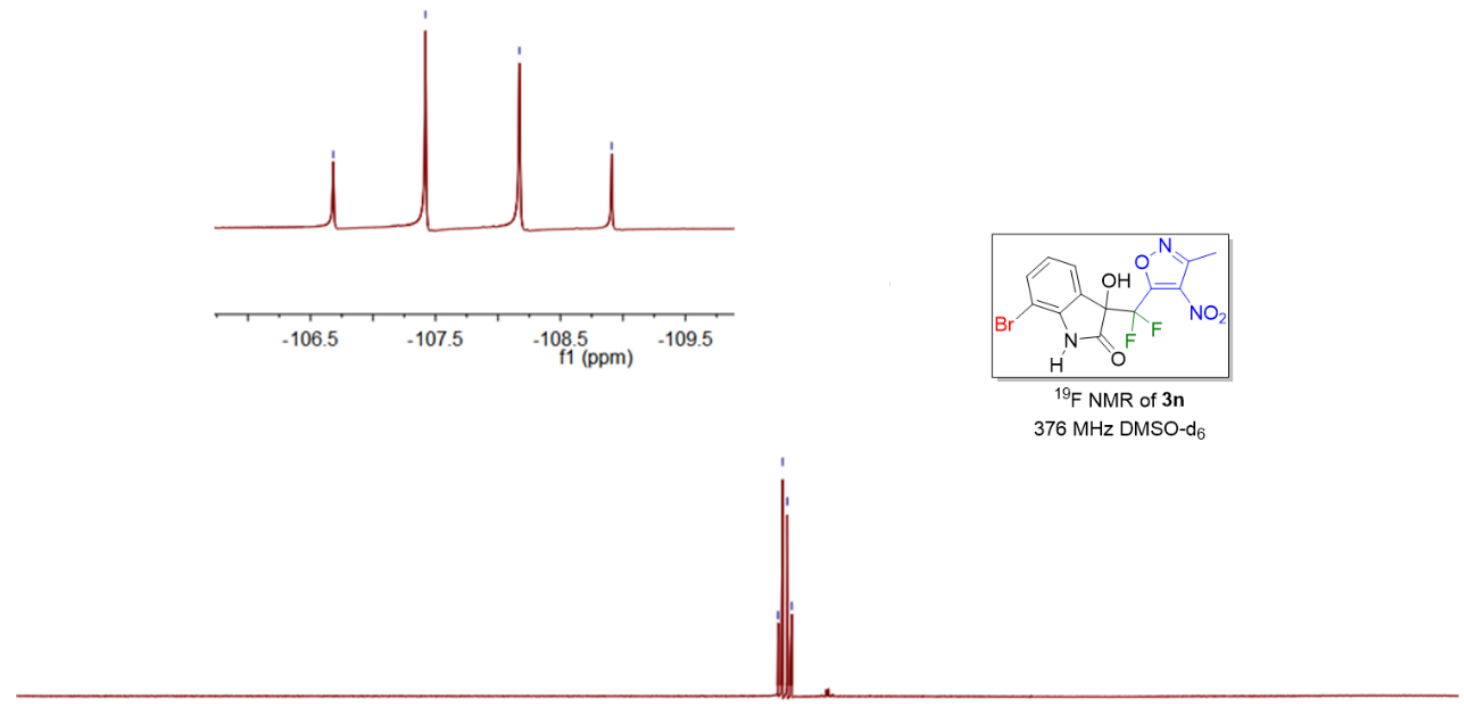

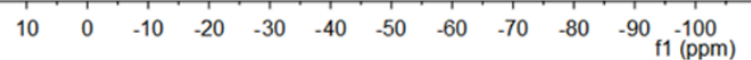

$\begin{array}{llllll}-120 & -140 & -160 & -180 & -200\end{array}$ 


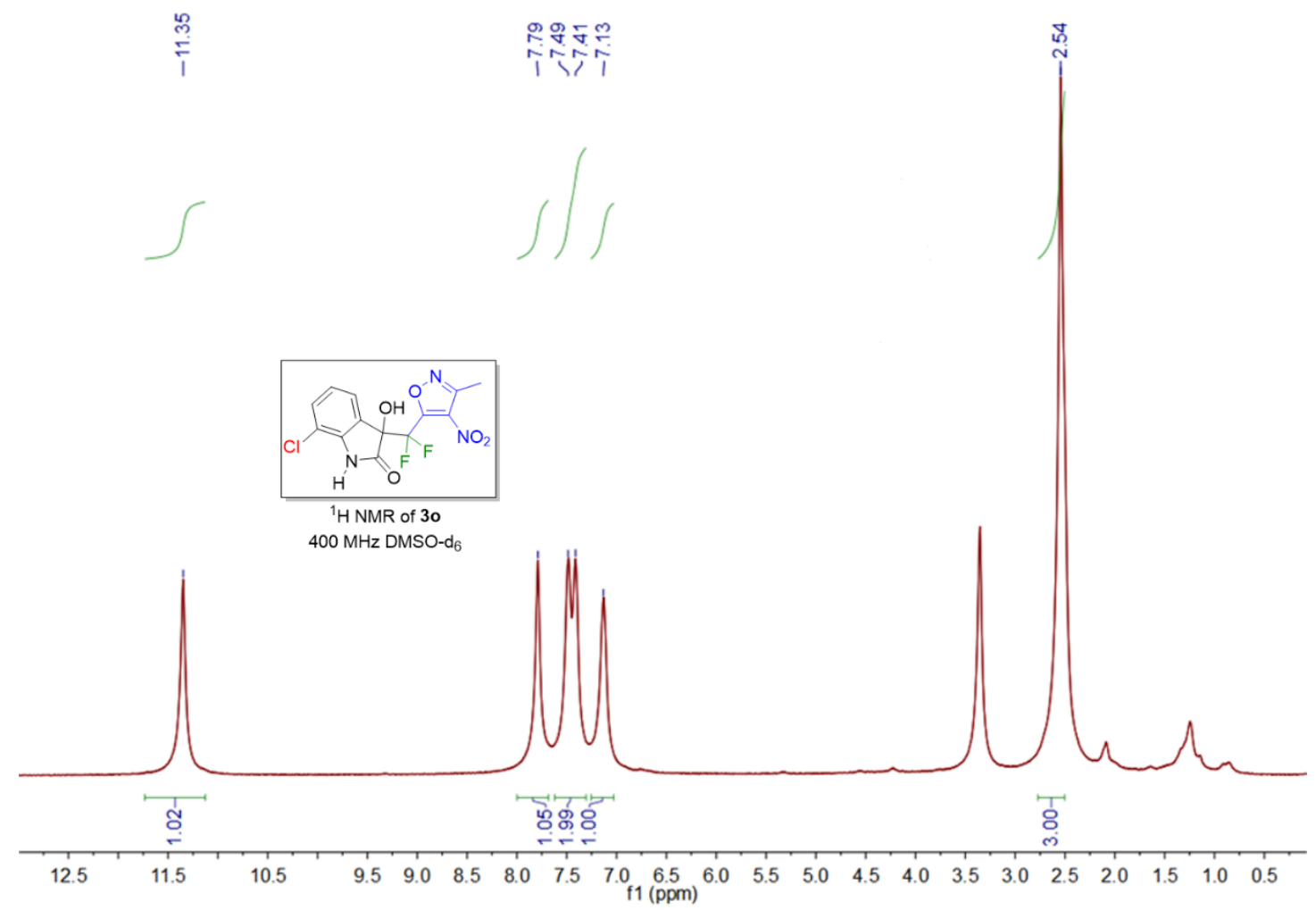

ل
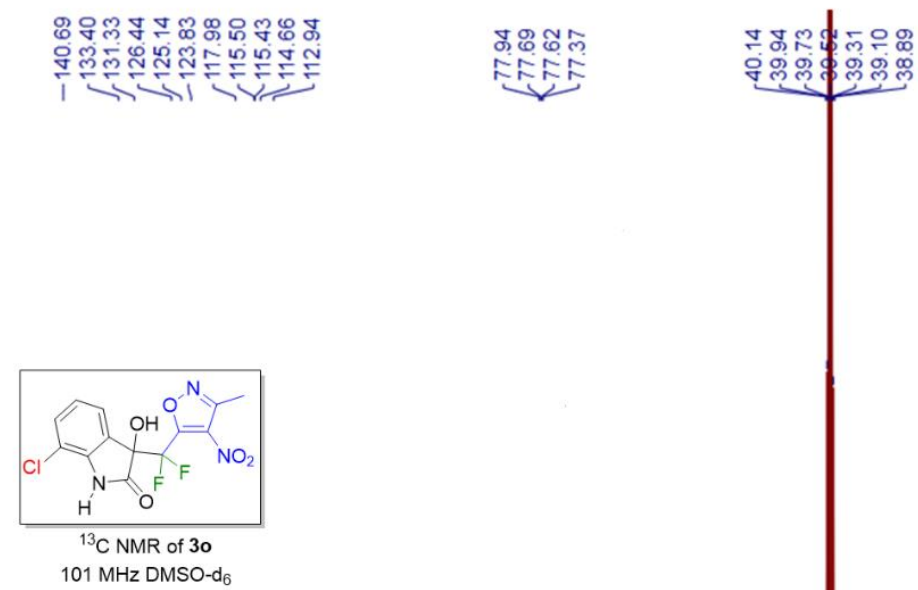

둥

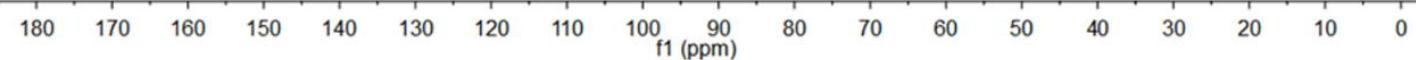



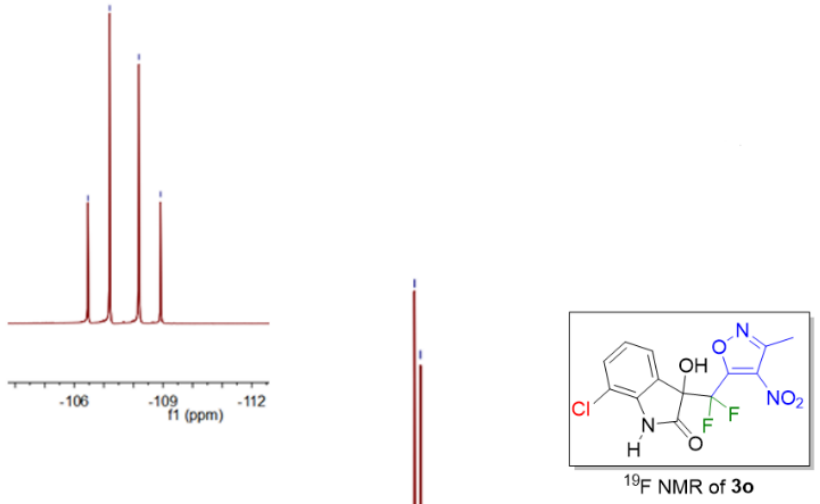

$376 \mathrm{MHz}$ DMSO-d

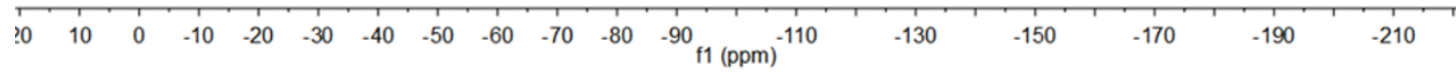

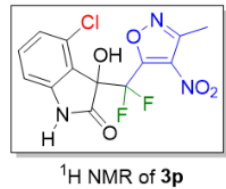

$400 \mathrm{MHz}$ DMSO-d

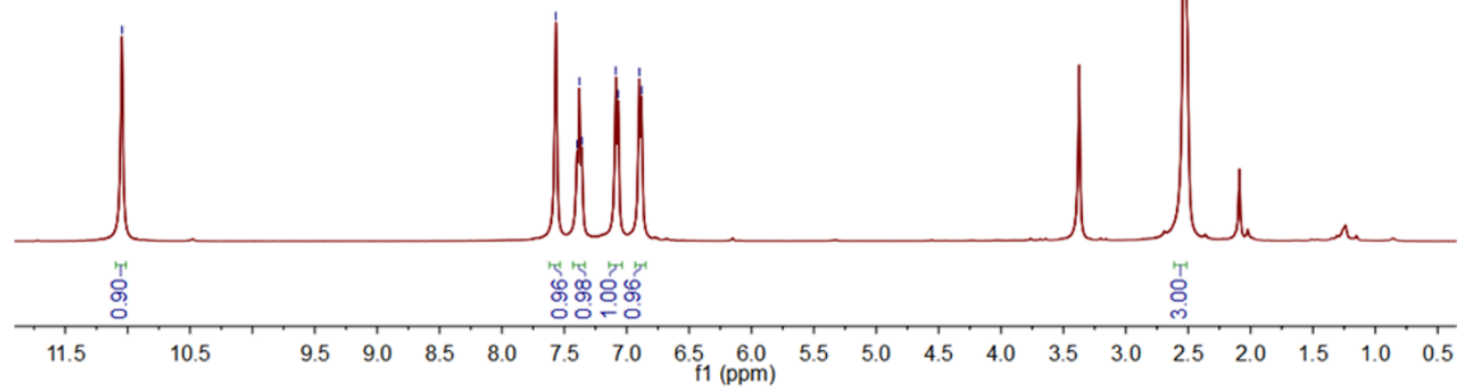




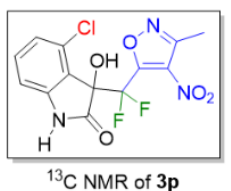
$101 \mathrm{MHz}$ DMSO-d 6

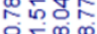
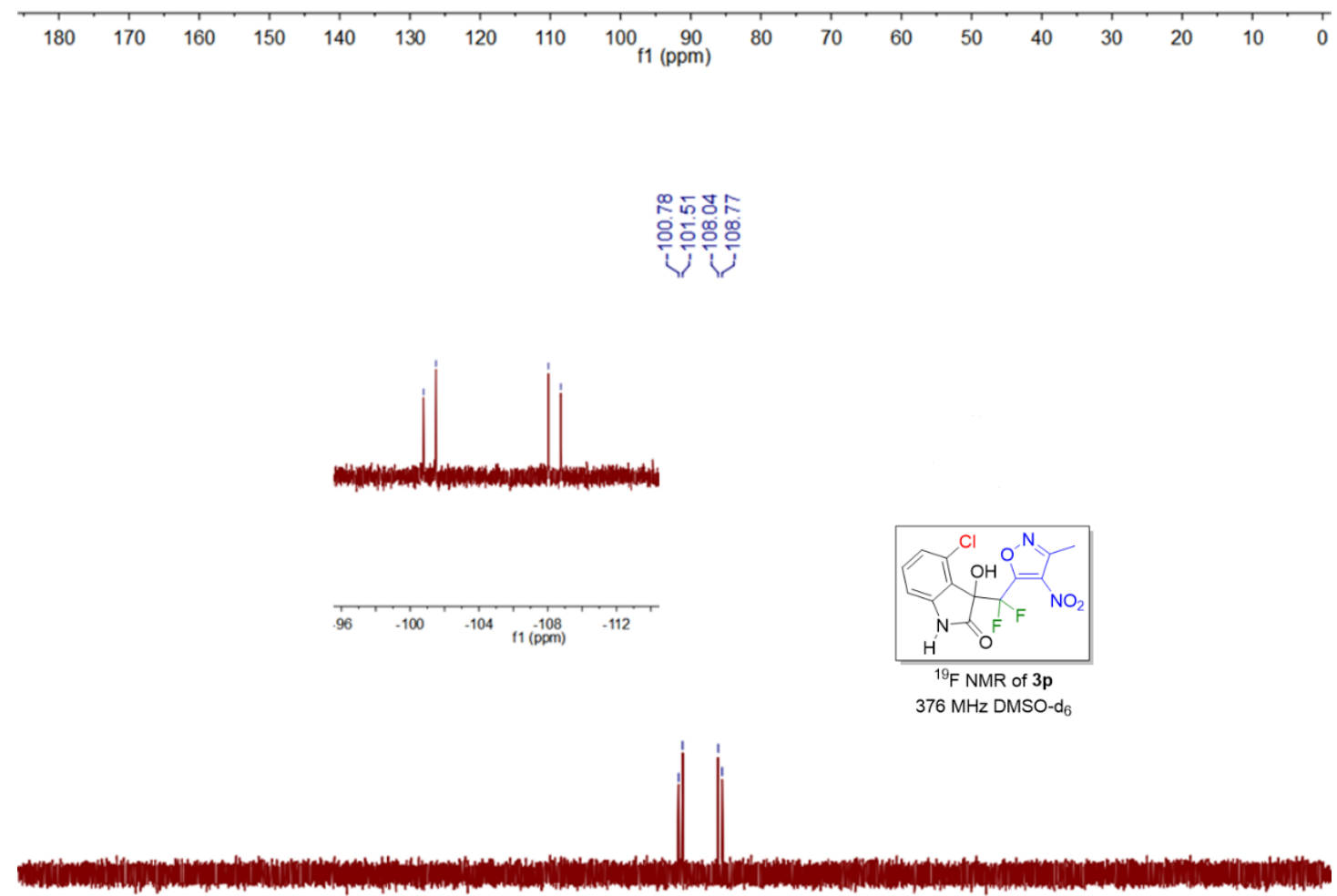

\begin{tabular}{llllllllllllllll}
\hline 0 & 10 & 0 & -10 & -20 & -30 & -40 & -50 & -60 & -70 & -80 & -90 & & \\
& $f 1(\mathrm{ppm})$ & -110 & -130 & -150 & -170 & -190 & -210
\end{tabular}




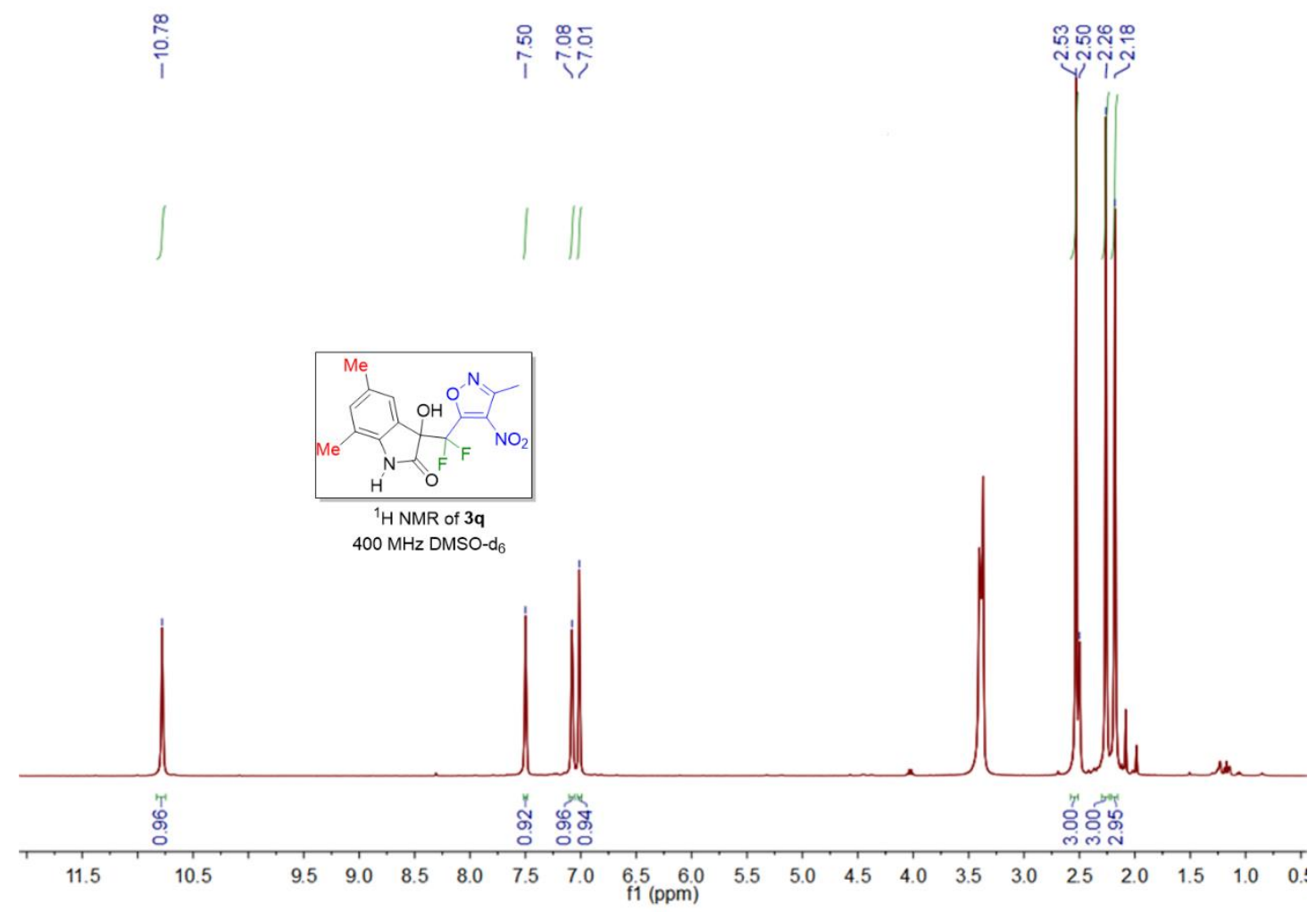

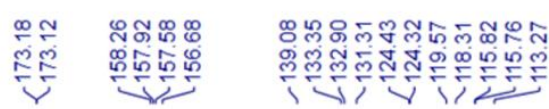

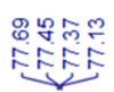
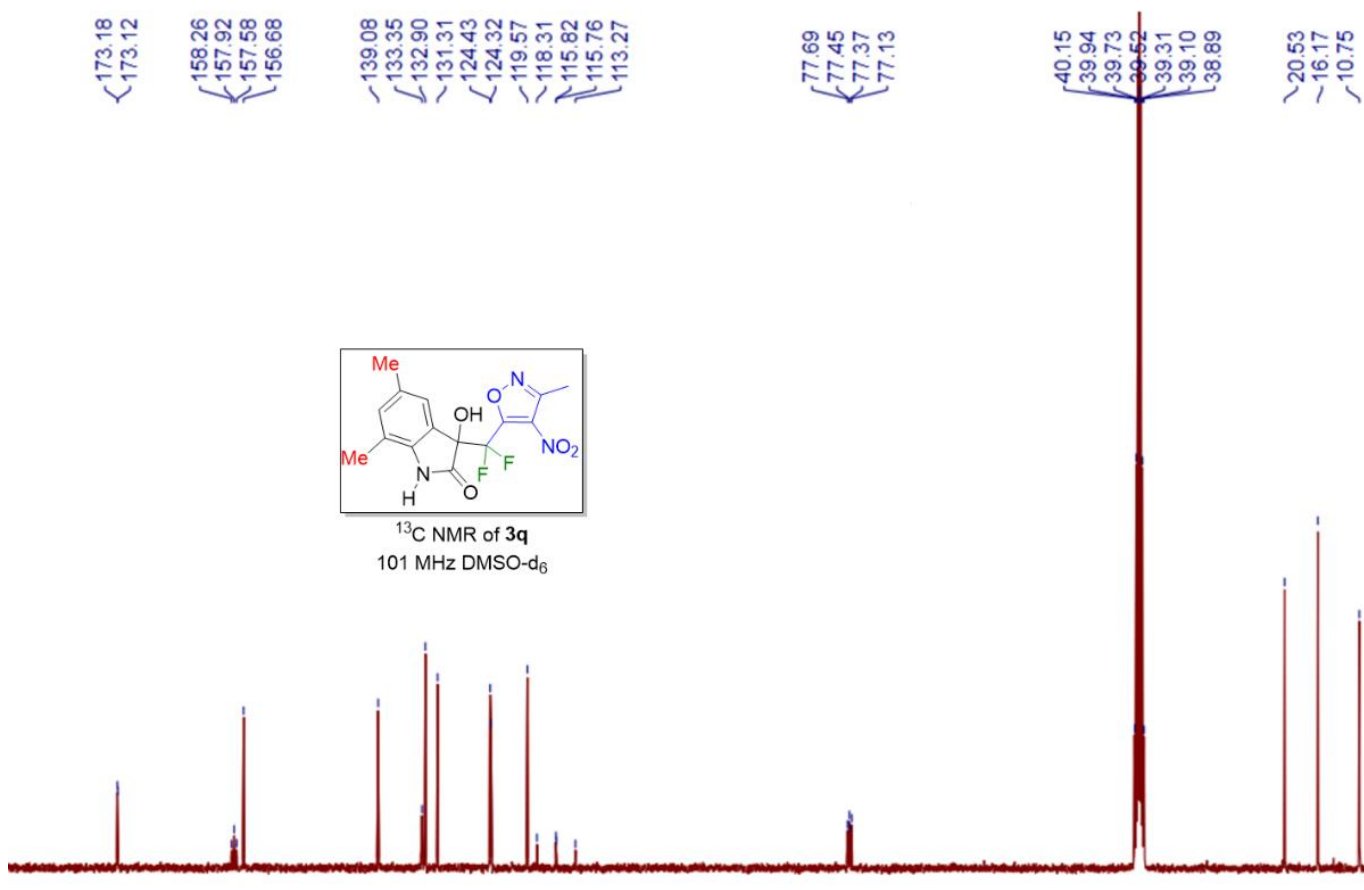

$\begin{array}{lllllllll}180 & 170 & 160 & 150 & 140 & 130 & 120 & 110 & 100 \\ \mathrm{f} 1(\mathrm{ppm})\end{array}$

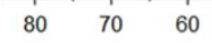

50

$\begin{array}{lllll}40 & 30 & 20 & 10 & 0\end{array}$ 


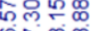

ำำํำ

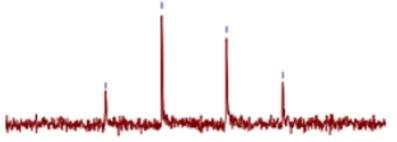

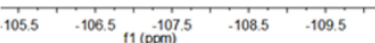

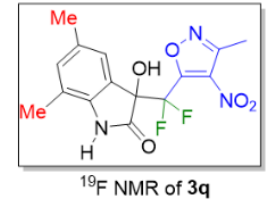

$376 \mathrm{MHz}$ DMSO-d

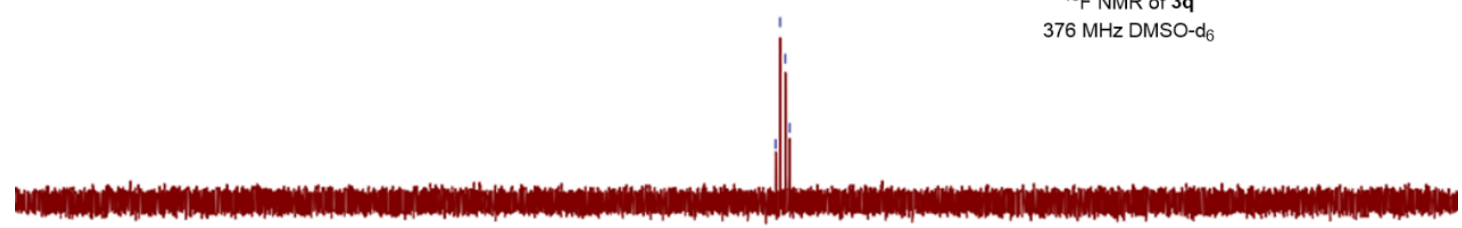

怘

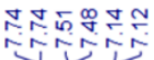

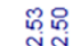

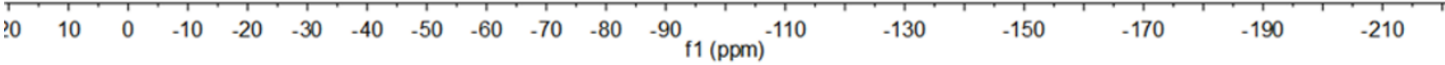

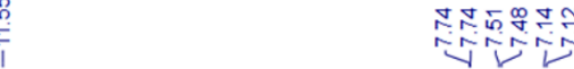

$1 / 1$

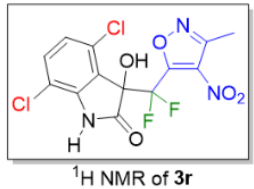

$400 \mathrm{MHz}$ DMSO-d

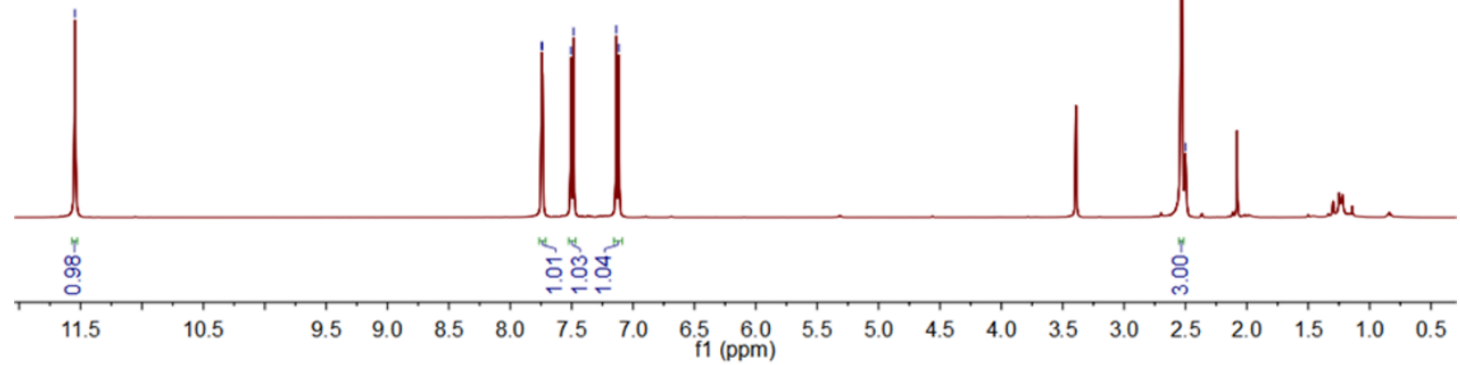



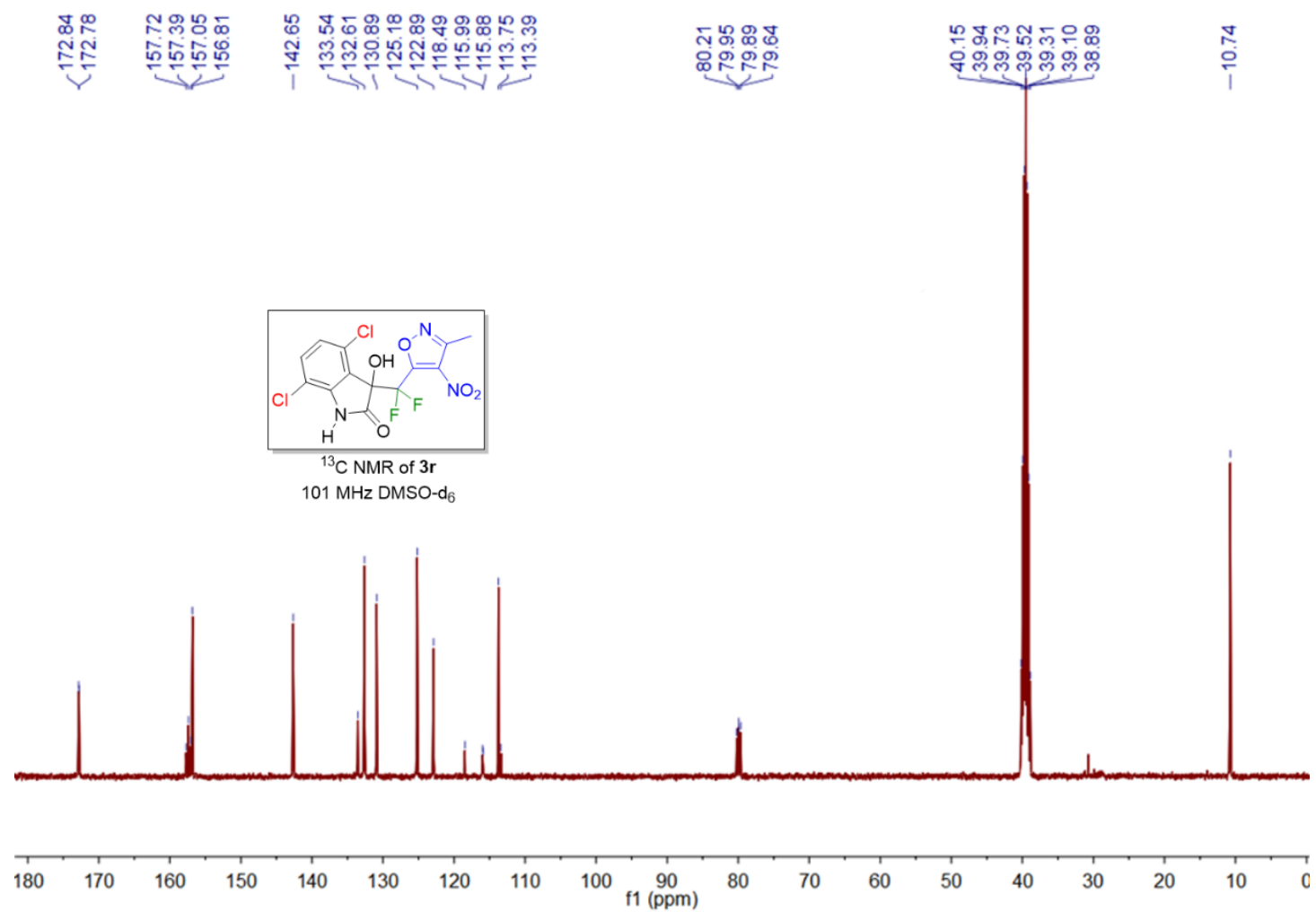

뮹ำ

훙ㅇㅇㅁำ

i
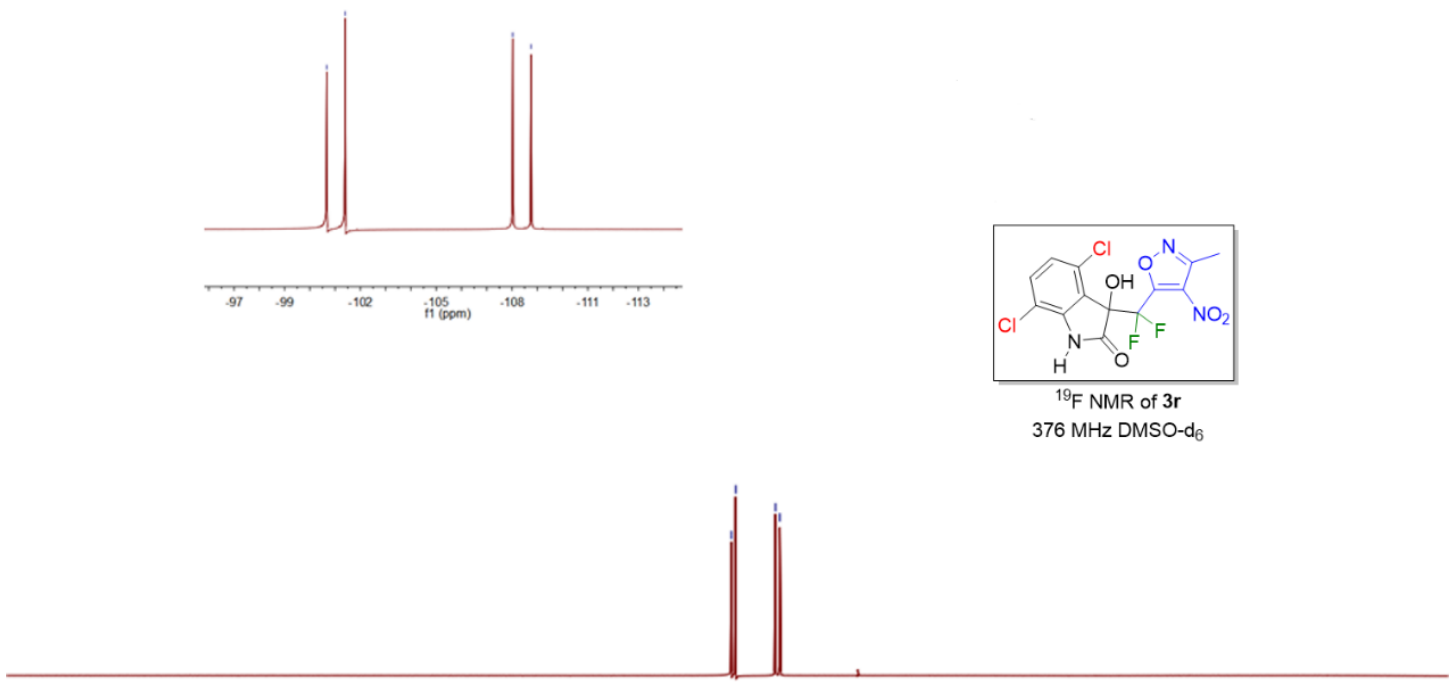

\begin{tabular}{lllllllllllllllllll}
\hline 0 & 10 & 0 & -10 & -20 & -30 & -40 & -50 & -60 & -70 & -80 & -90 & & -110 & -130 & -150 & -170 & -190 & -210
\end{tabular} 


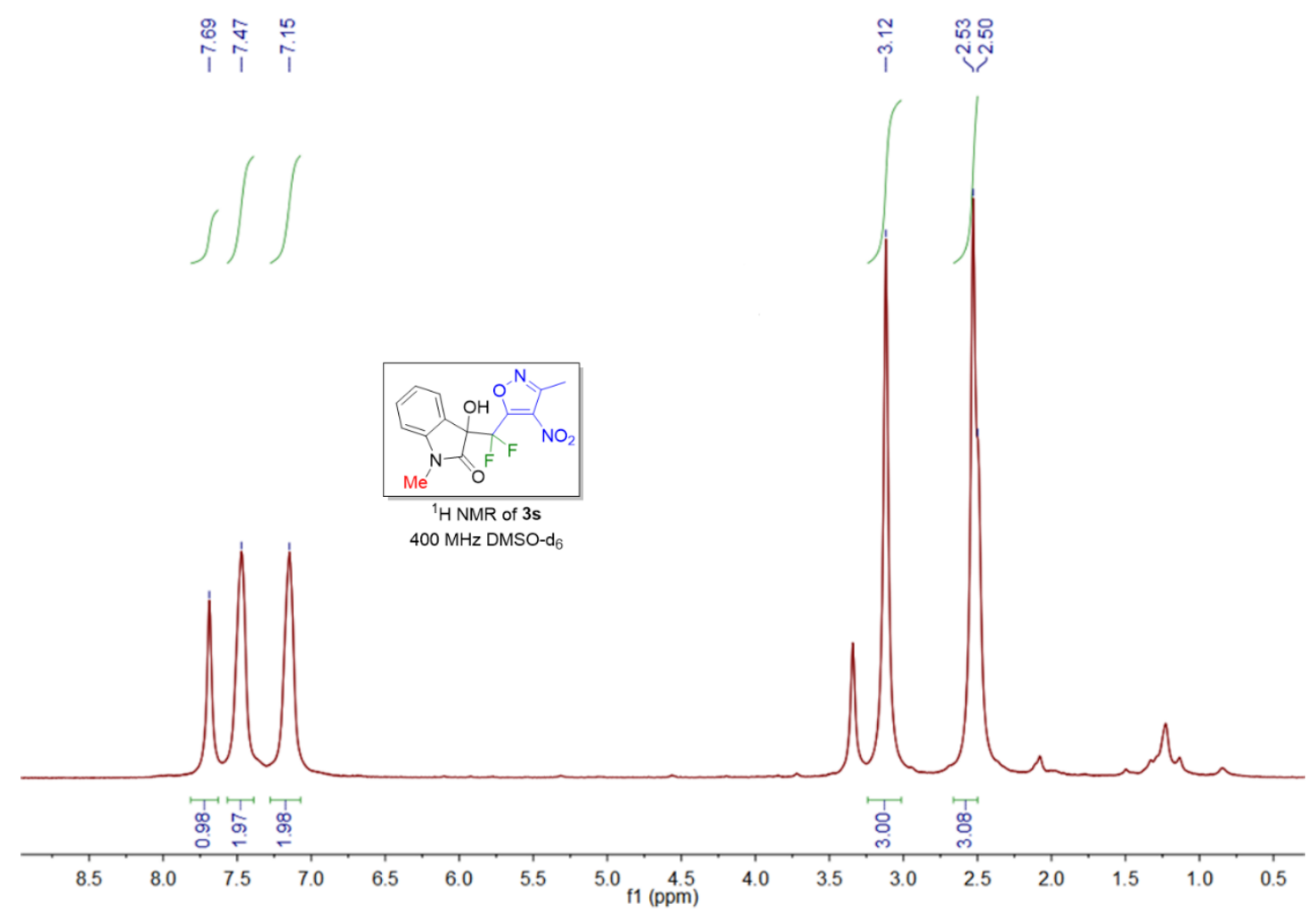

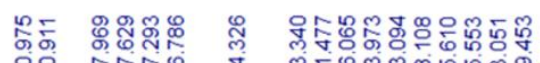

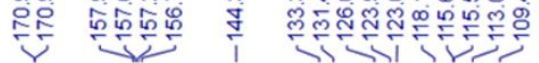

นึฏ

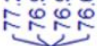

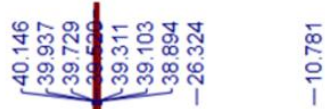

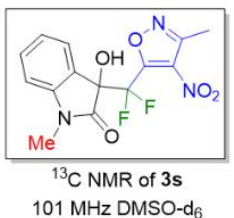

$101 \mathrm{MHz}$ DMSO-d

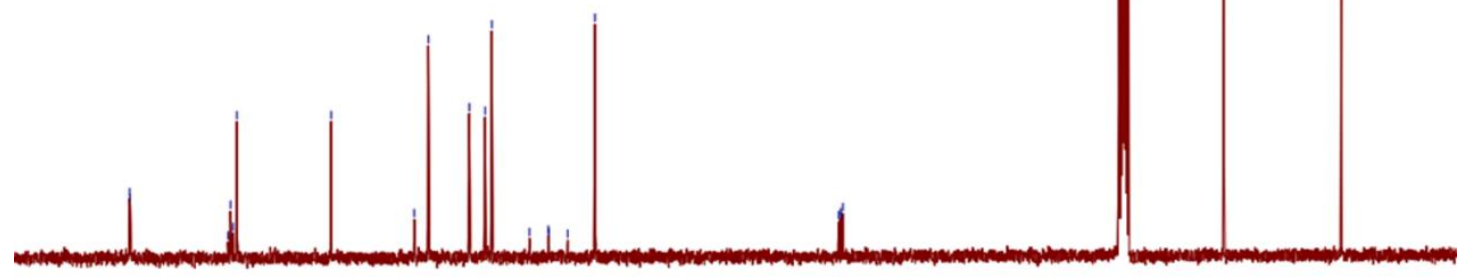

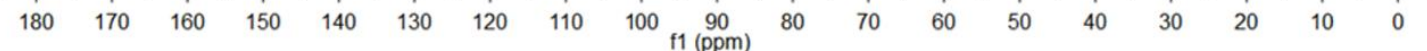


ㅉํำ

은ㅎํㅇํำ
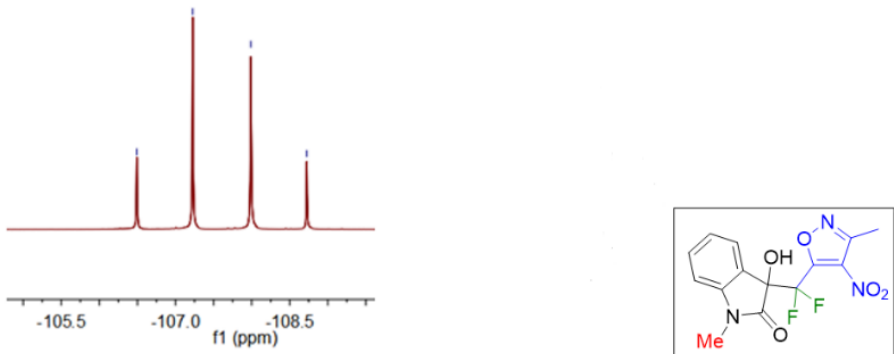

${ }^{19} \mathrm{~F}$ NMR of $3 \mathrm{~s}$ $376 \mathrm{MHz}$ DMSO-d 6

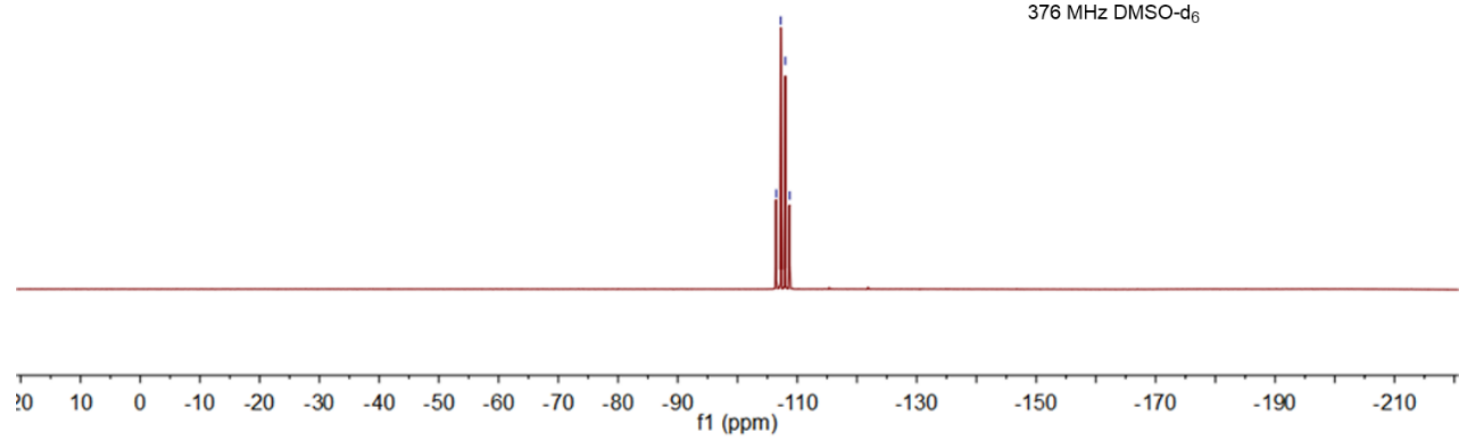

옹ㅁํㄴ

NiN

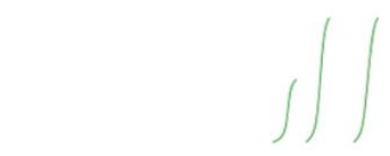

लूल्भल
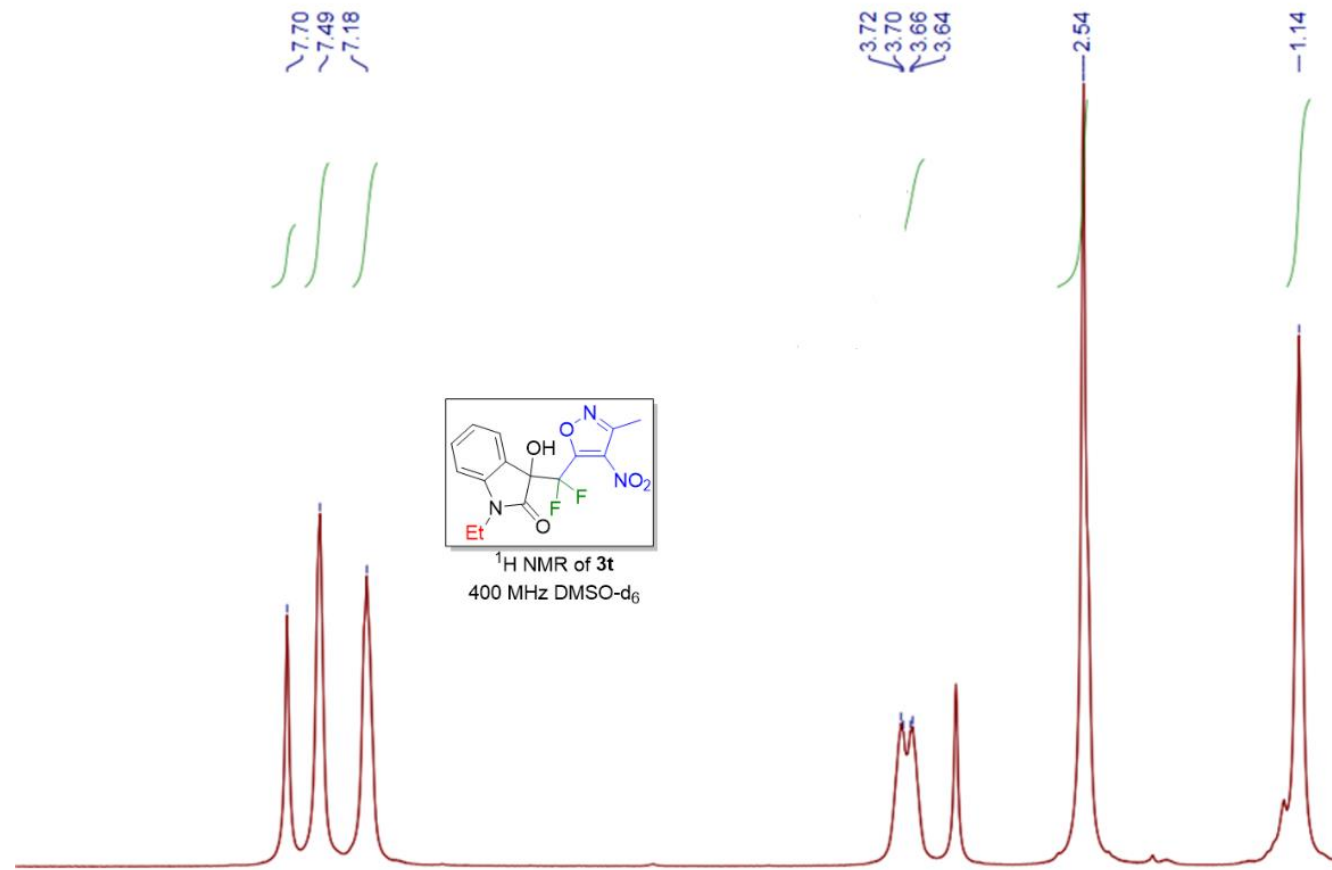

요요

ㄴํㅇ
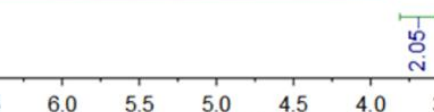

'잉

$\begin{array}{llllllllllllllllll}9.0 & 8.5 & 8.0 & 7.5 & 7.0 & 6.5 & 6.0 & 5.5 & \begin{array}{c}1 \\ \mathrm{f} 1\end{array}(\mathrm{ppm}) & 4 & 4.0 & 3.5 & 3.0 & 2.5 & 2.0 & 1.5 & 1.0 & 0.5\end{array}$

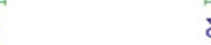



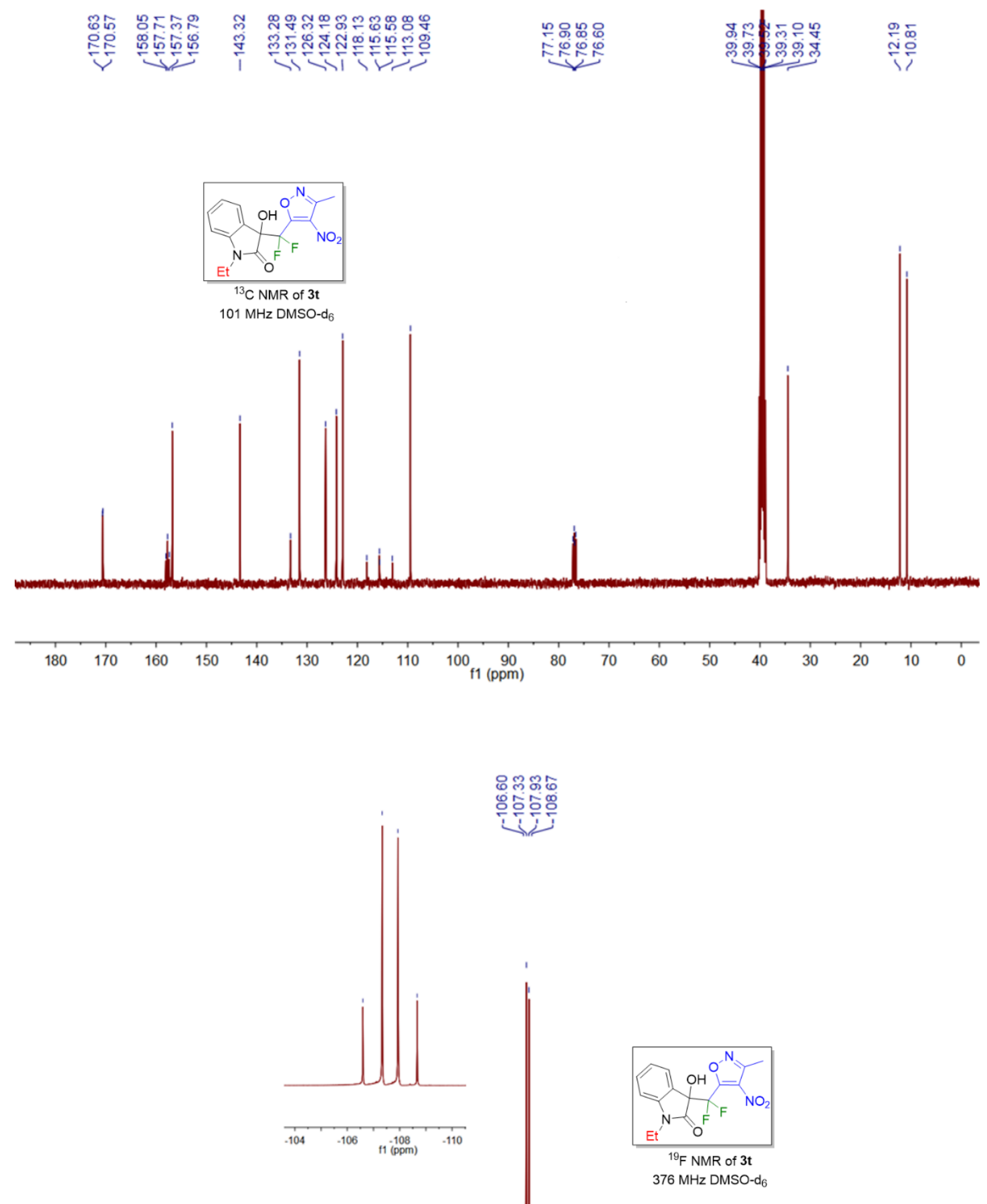

\begin{tabular}{lllllllllllllllllll}
\hline 0 & 10 & 0 & -10 & -20 & -30 & -40 & -50 & -60 & -70 & -80 & -90 & -110 & -130 & -150 & -170 & -190 & -210
\end{tabular} 


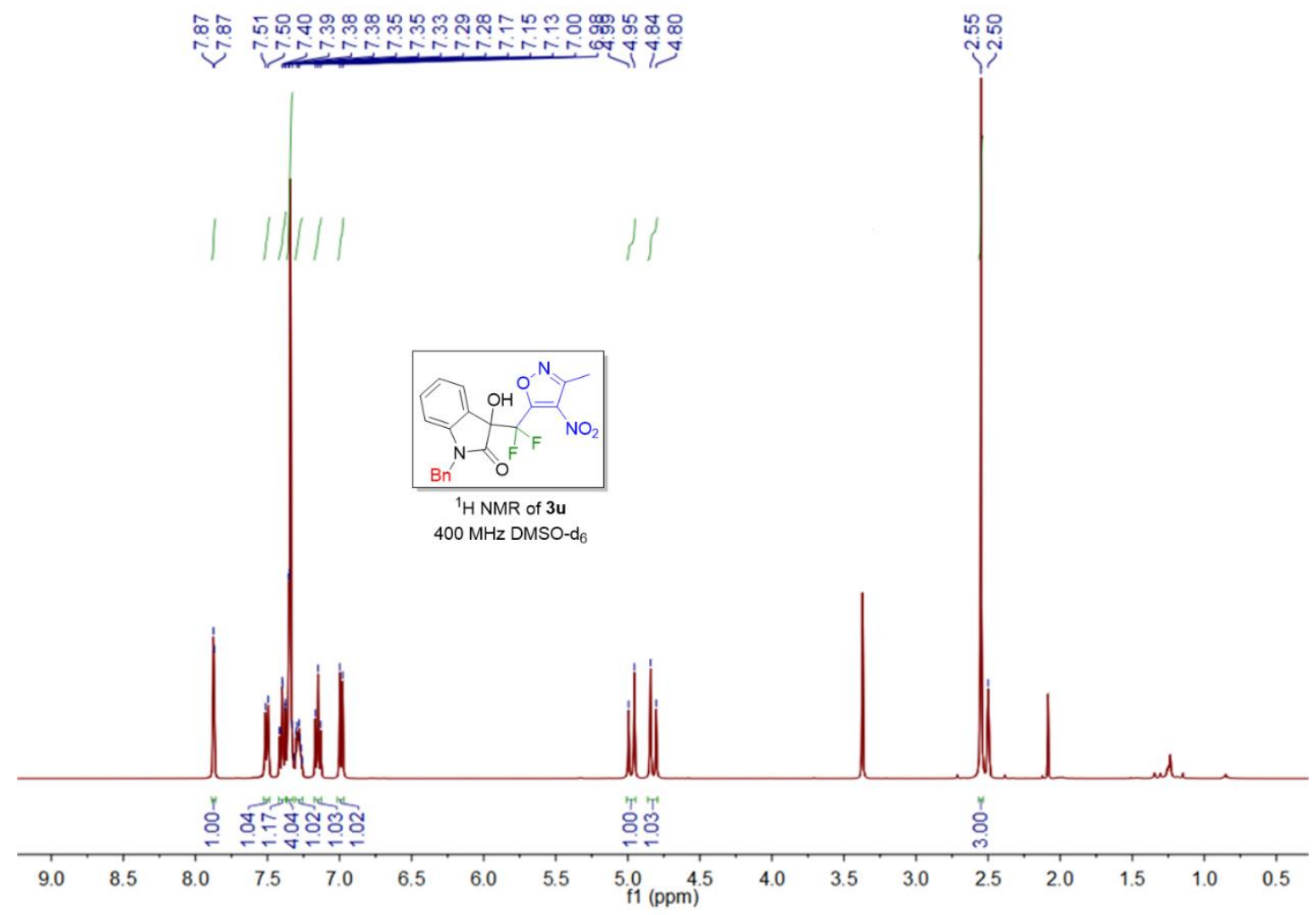

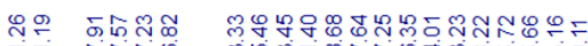

-

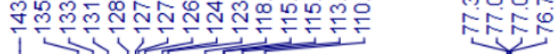

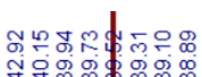

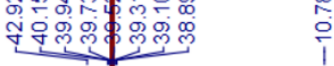

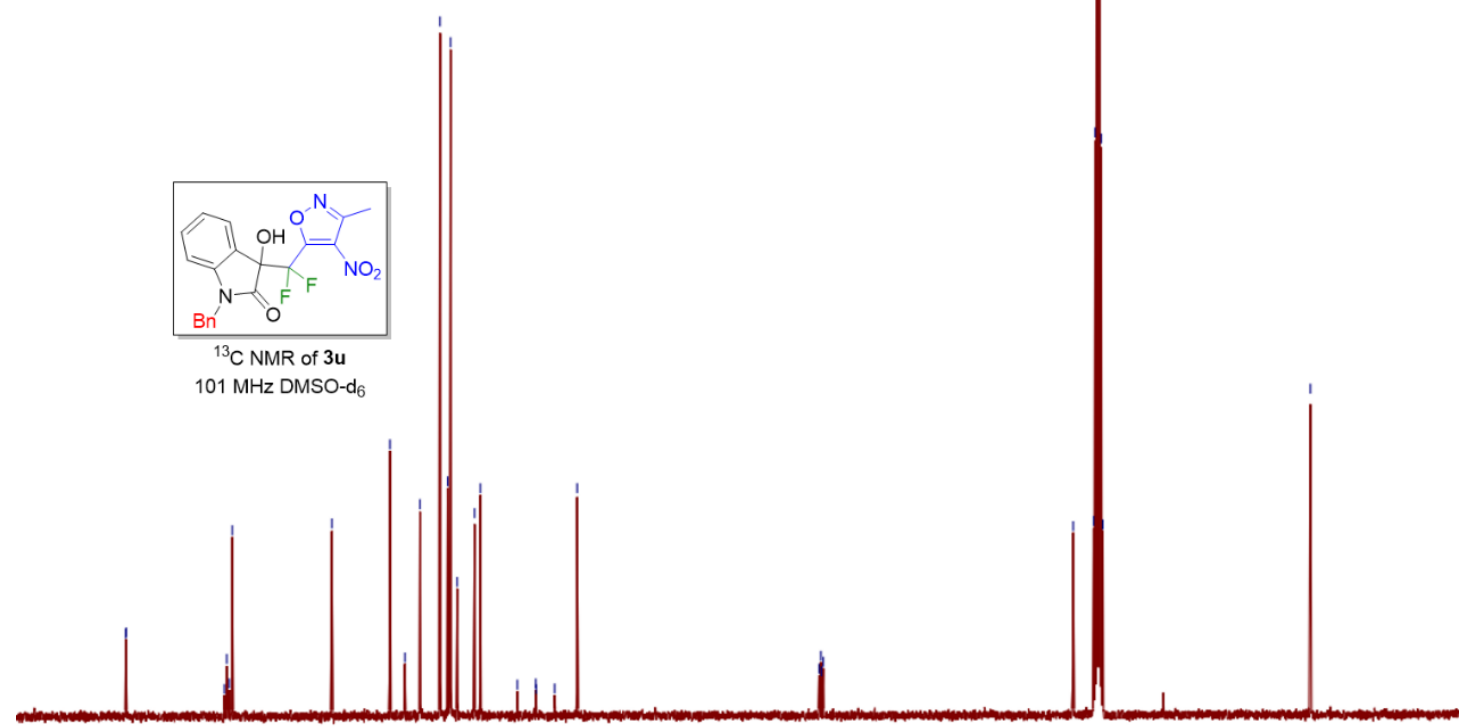

$\begin{array}{lllllllllllllllllll}180 & 170 & 160 & 150 & 140 & 130 & 120 & 110 & 100 & \begin{array}{c}90 \\ \mathrm{f} 1(\mathrm{ppm})\end{array} & 80 & 70 & 60 & 50 & 40 & 30 & 20 & 10 & 0\end{array}$ 

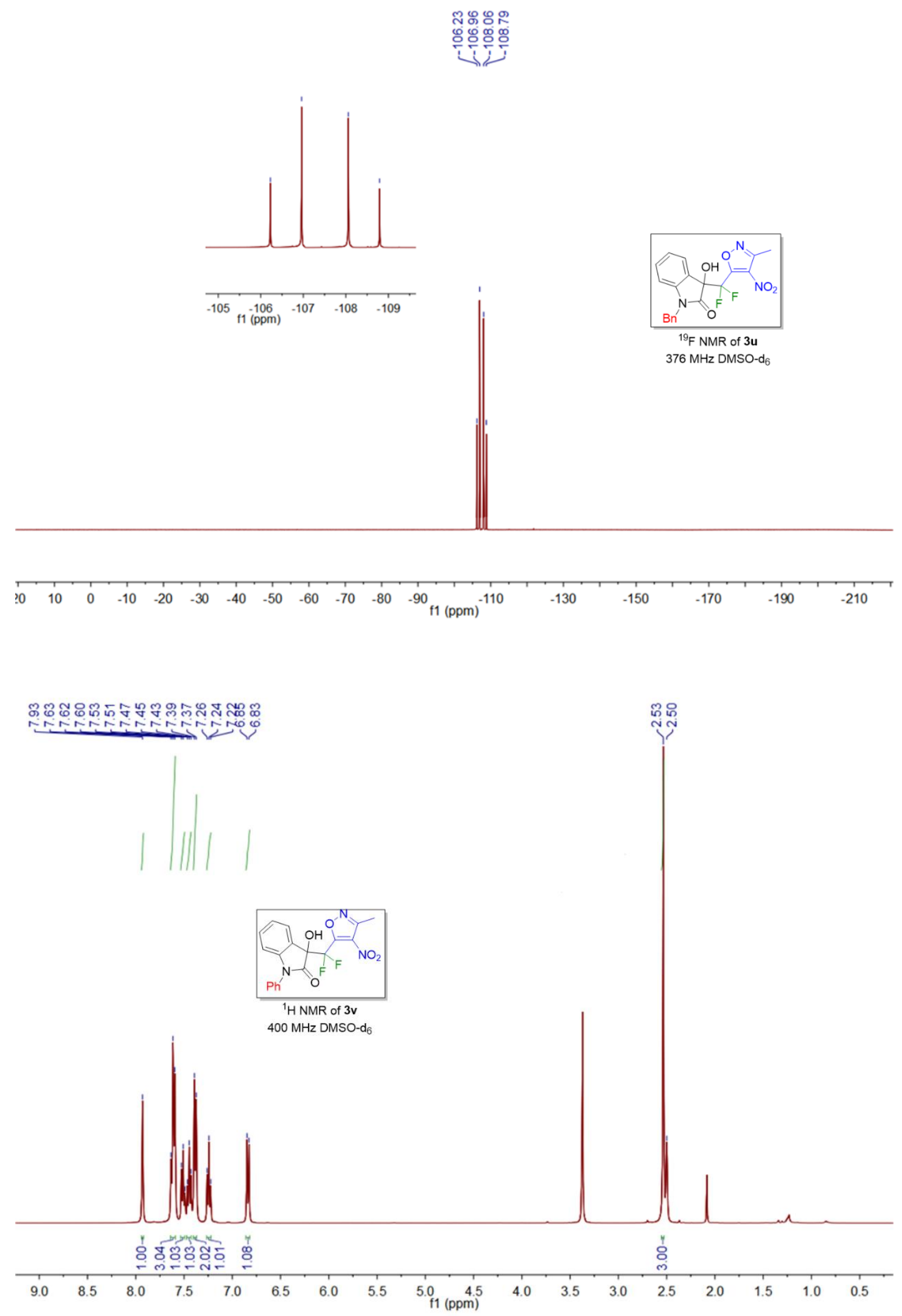

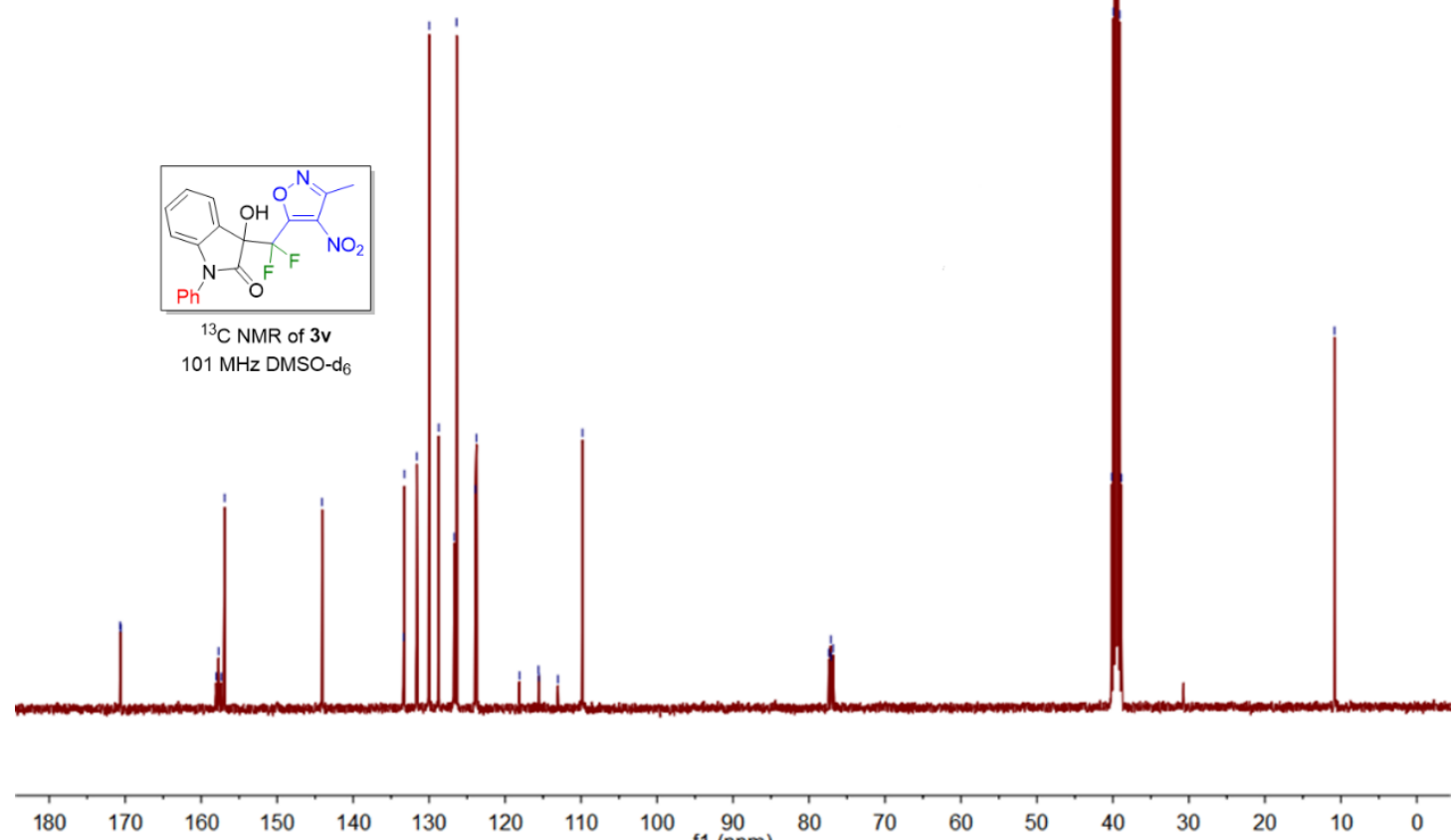

$130 \quad 1$

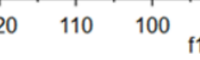

f1 $\stackrel{90}{(\mathrm{ppm})}$

80

50

$40 \quad 30$

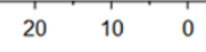

必

ํํㅇํํㅇำ

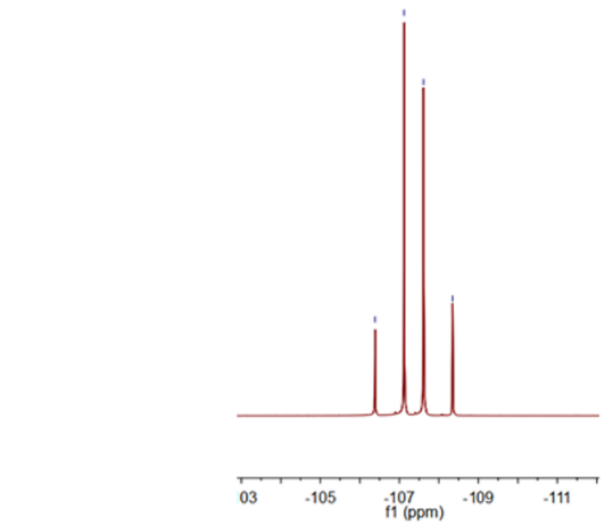

은은은

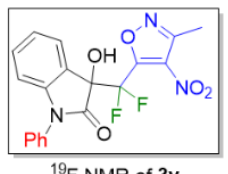

$376 \mathrm{MHz}$ DMSO-d 6

\begin{tabular}{llllllllllll}
\hline 0 & 10 & 0 & -10 & -20 & -30 & -40 & -50 & -60 & -70 & -80 & -90 \\
$\mathrm{f} 1(\mathrm{ppm})$ & -110
\end{tabular}

$-130$

$-150$

$190 \quad-210$ 

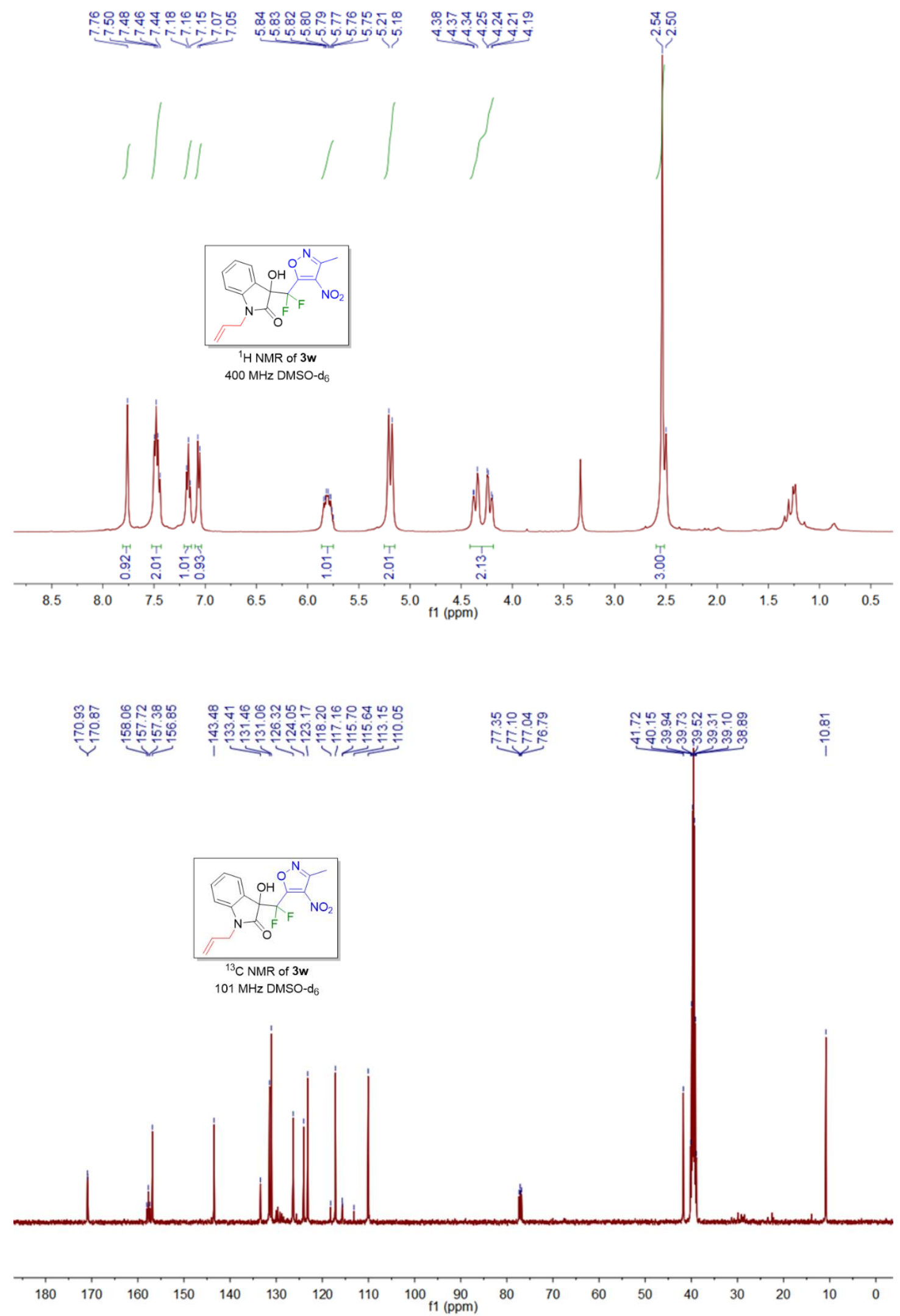


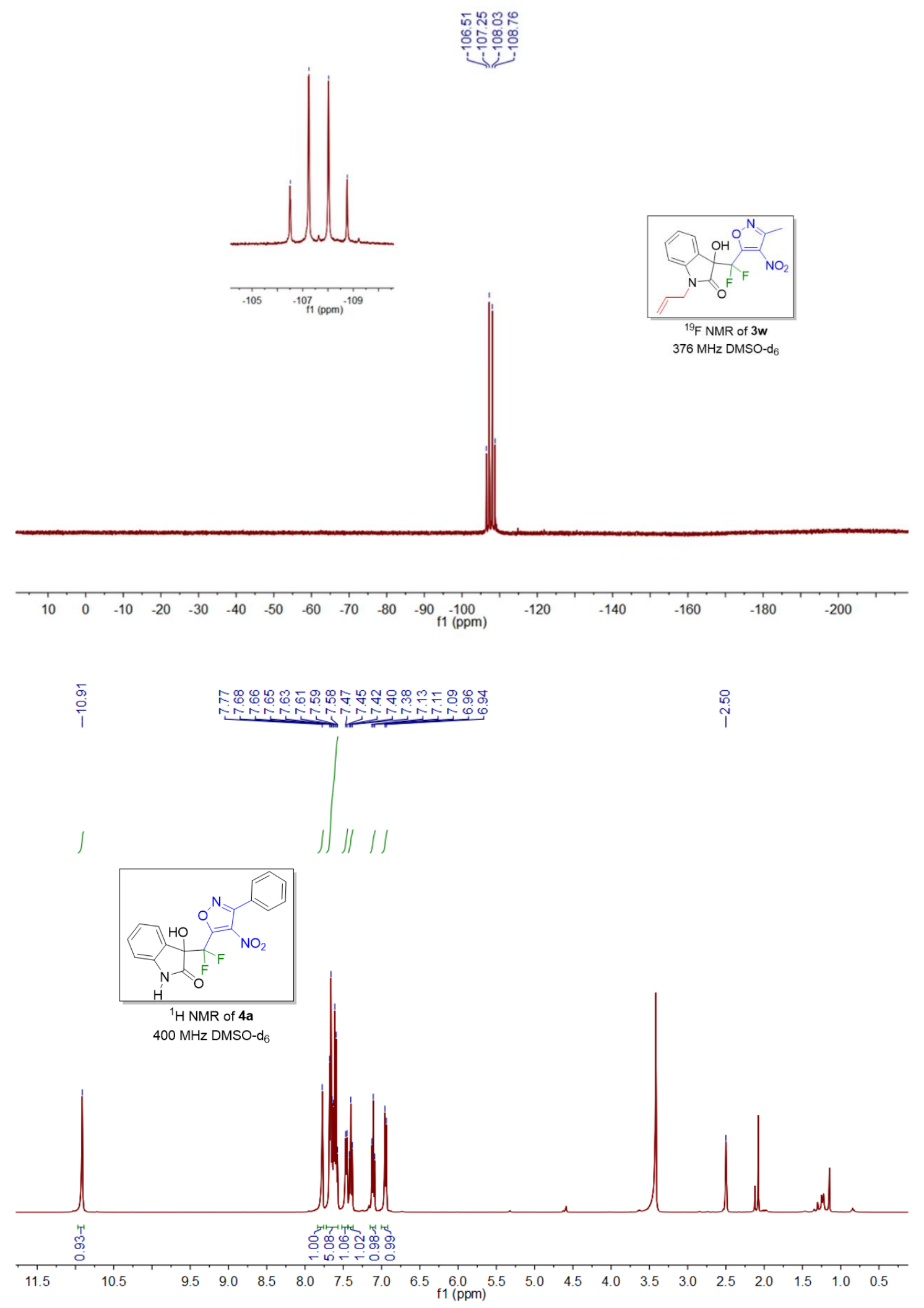



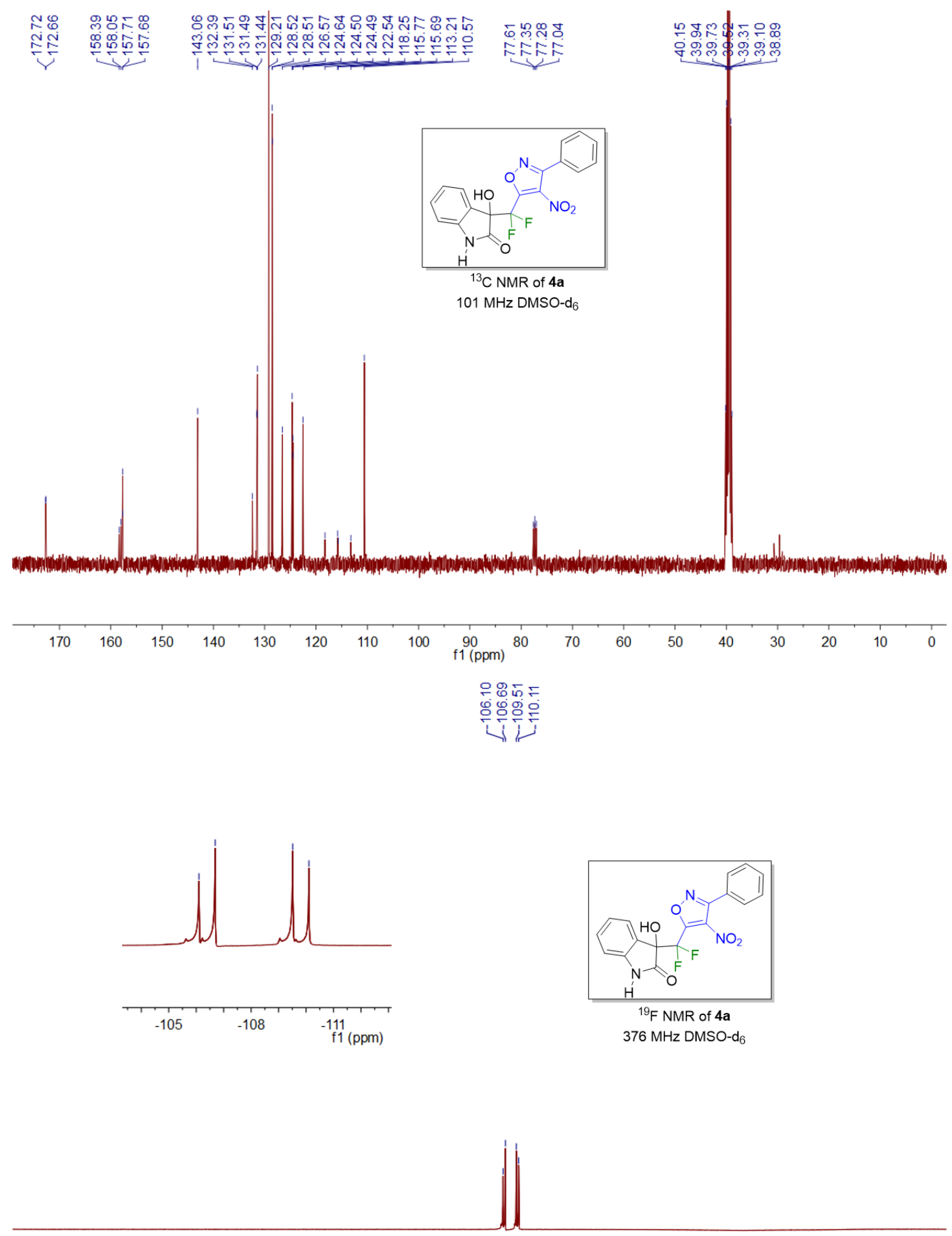

\begin{tabular}{lllllllllllllllllll}
\hline 0 & 10 & 0 & -10 & -20 & -30 & -40 & -50 & -60 & -70 & -80 & -90 & & 110 & -130 & -150 & -170 & -190 & -210
\end{tabular} 


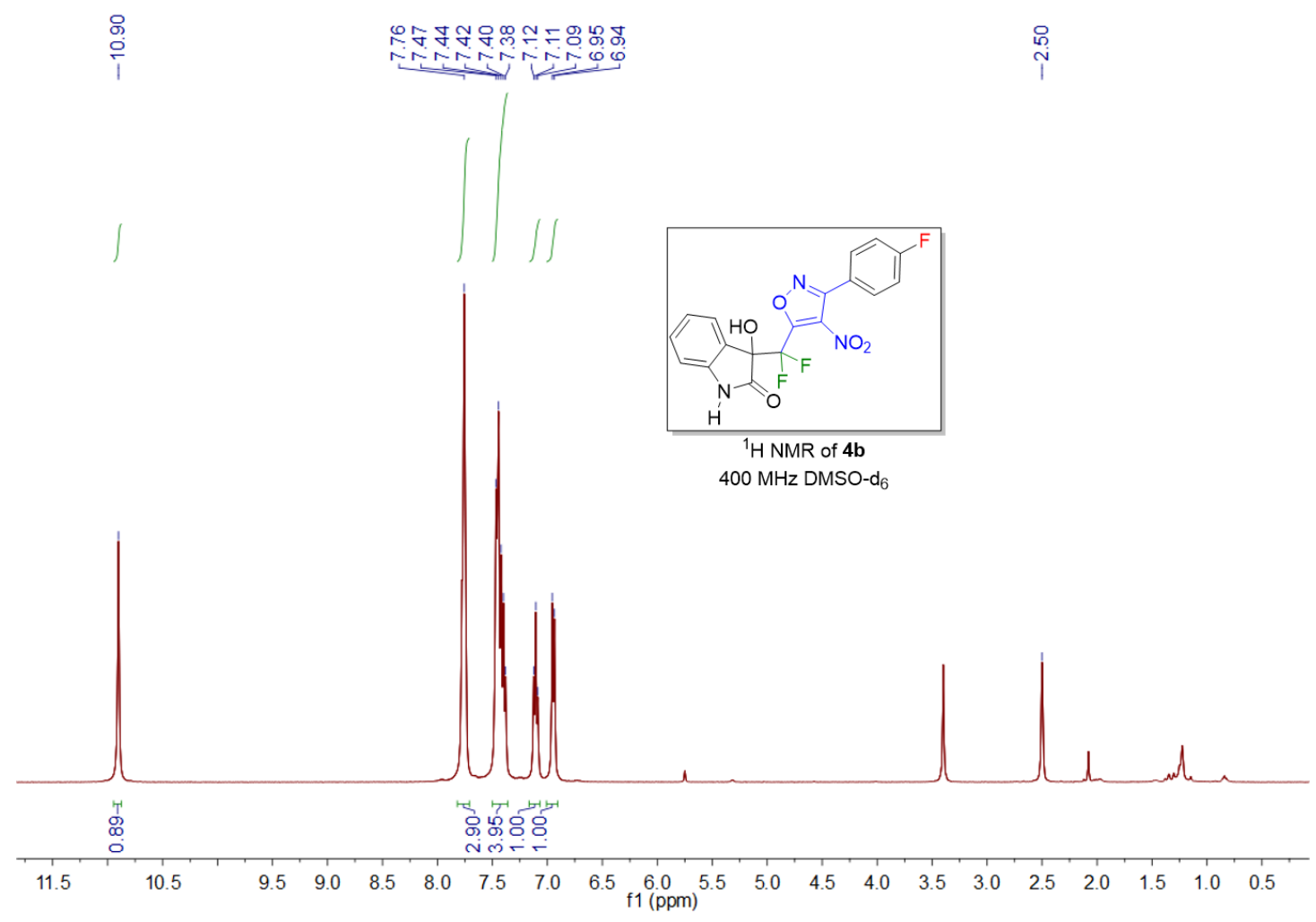

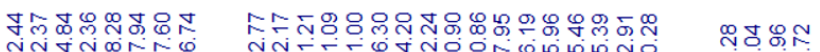

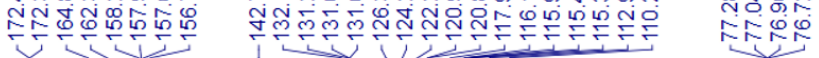

$\infty$

శ్ల

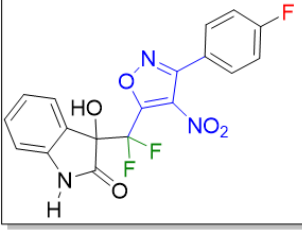

${ }^{13} \mathrm{C}$ NMR of $4 \mathrm{~b}$

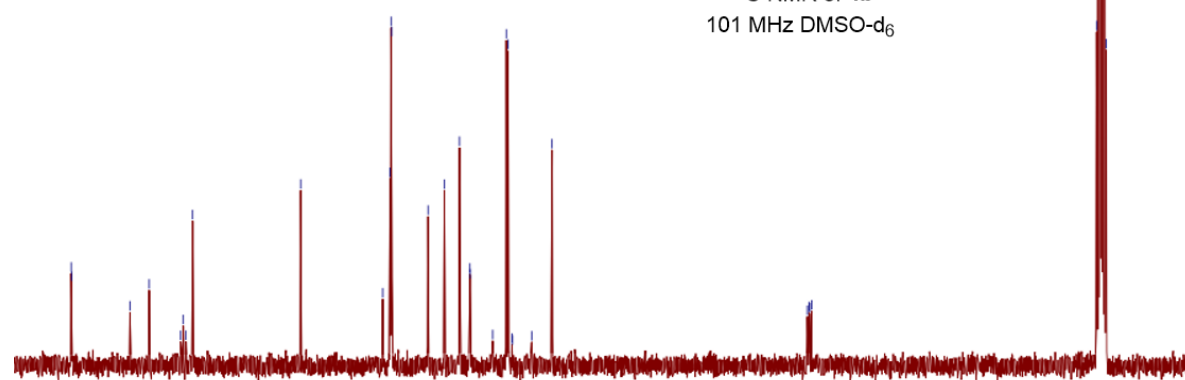

$\begin{array}{lllllllll}170 & 160 & 150 & 140 & 130 & 120 & 110 & 100 & \underset{\mathrm{f} 1}{90}(\mathrm{ppm})\end{array}$

$\begin{array}{llllllll}70 & 60 & 50 & 40 & 30 & 20 & 10 & 0\end{array}$ 


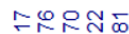

으응응음

近
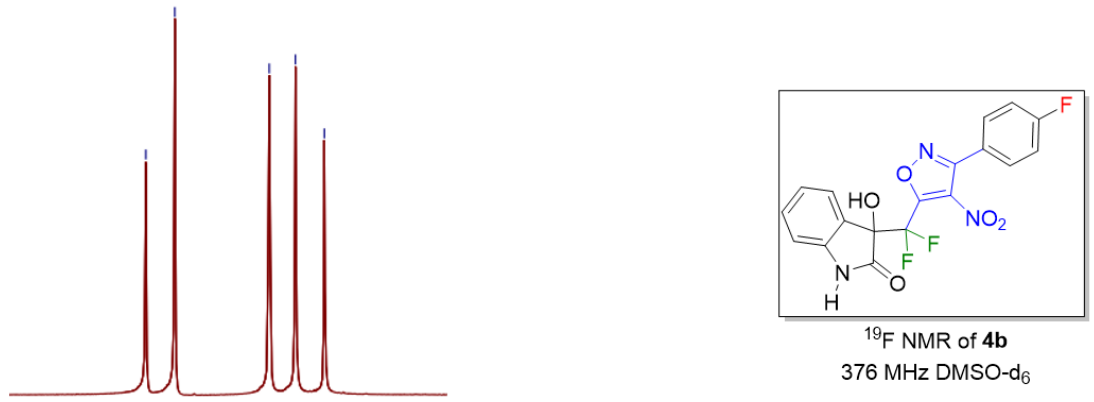

${ }^{19} \mathrm{~F} \mathrm{NMR}$ of $4 \mathrm{~b}$ $376 \mathrm{MHz}$ DMSO-d 6
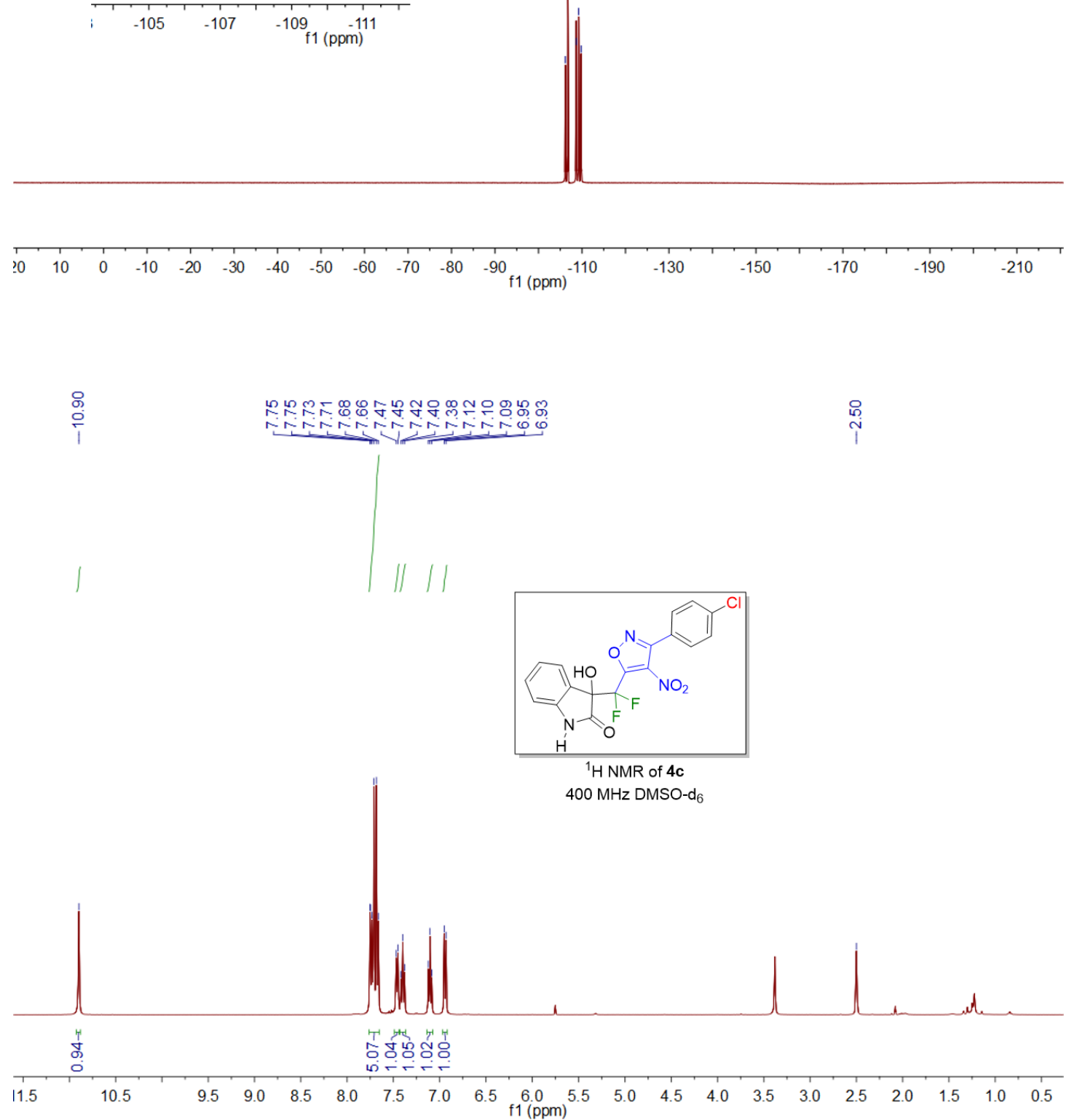

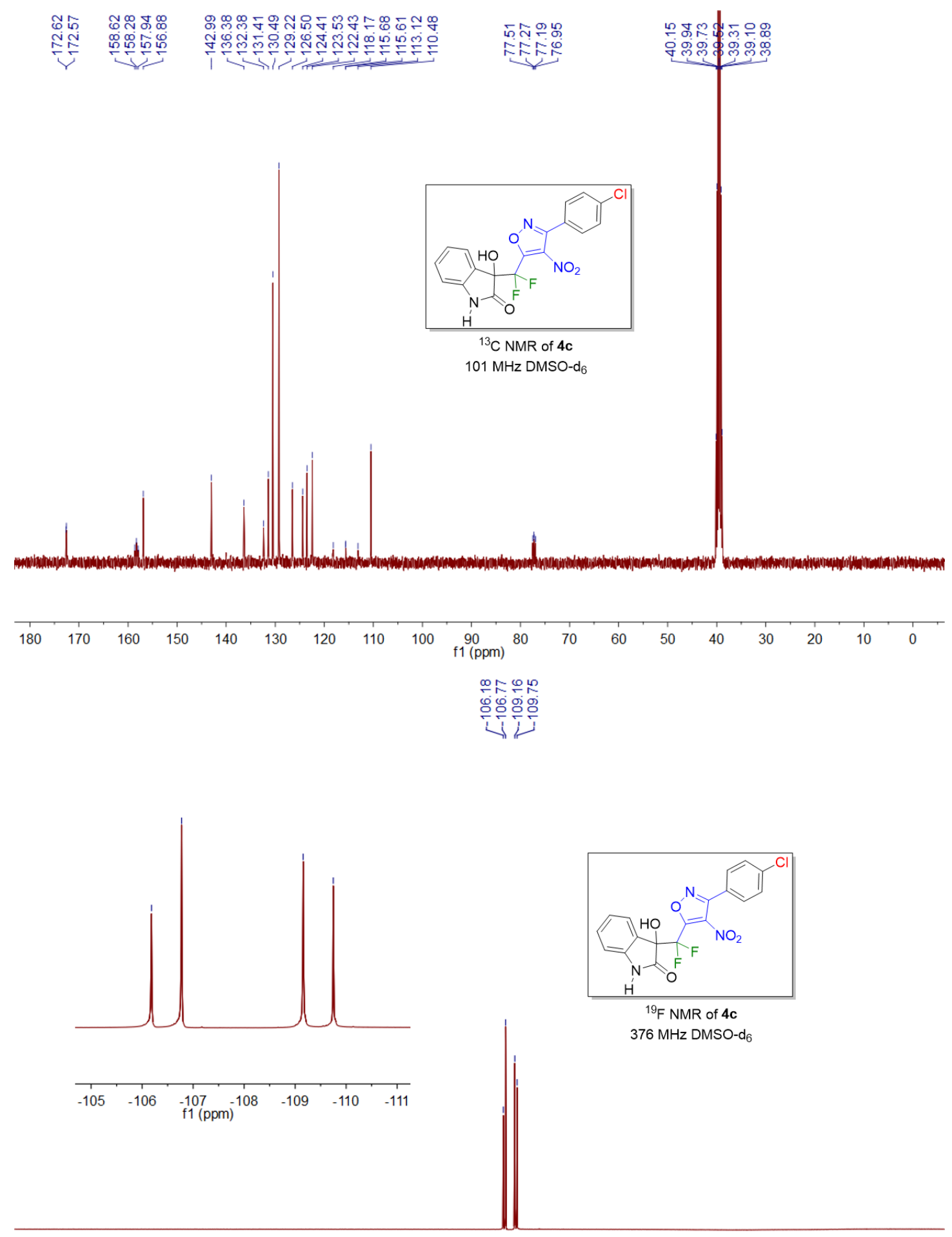

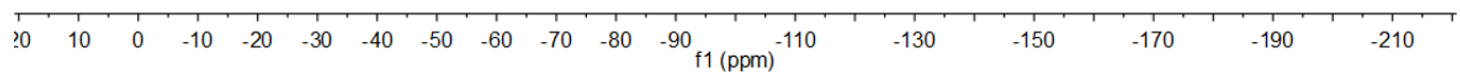




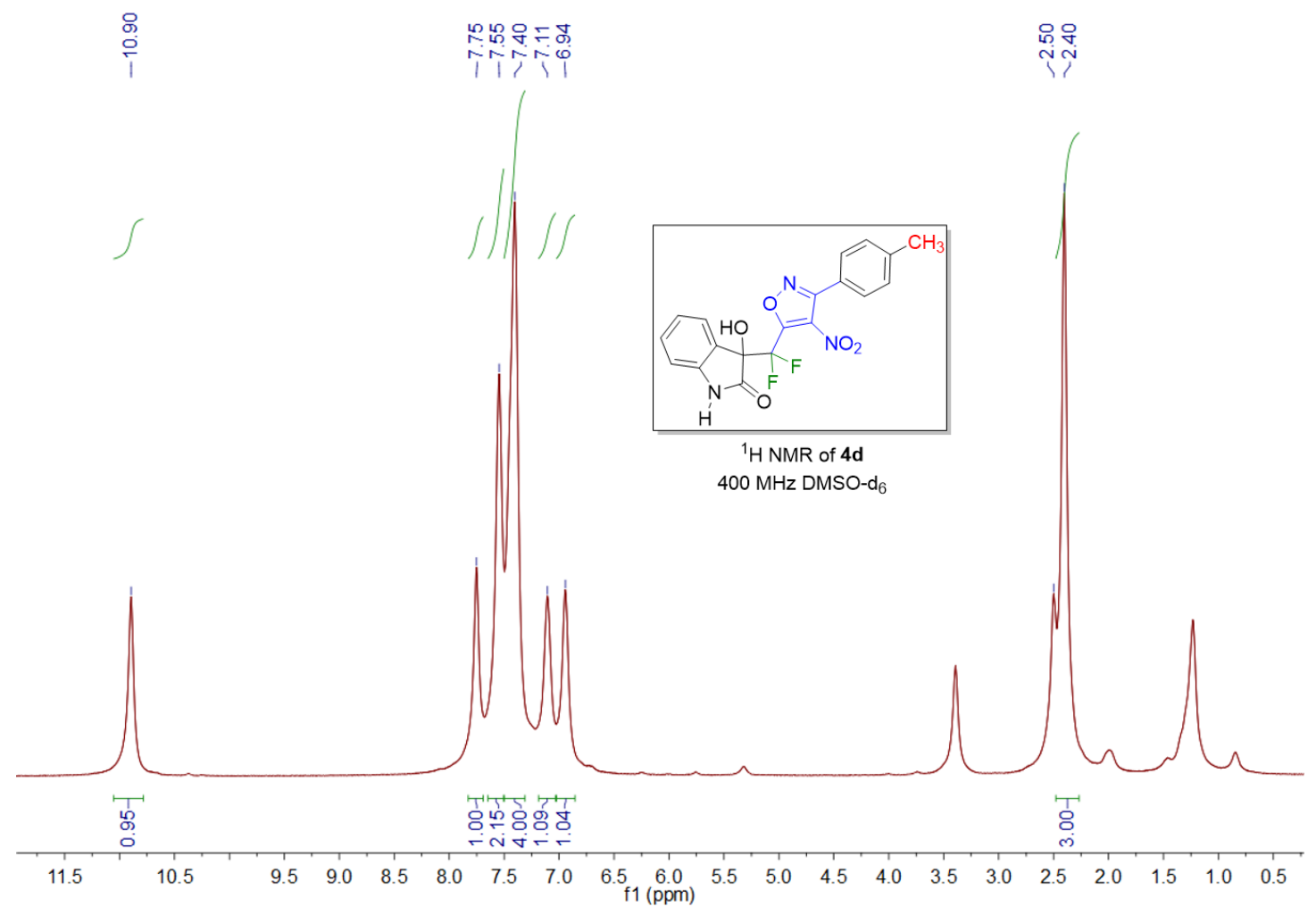

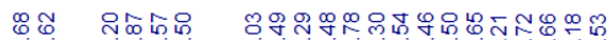

( )

Nine

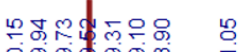

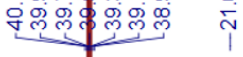

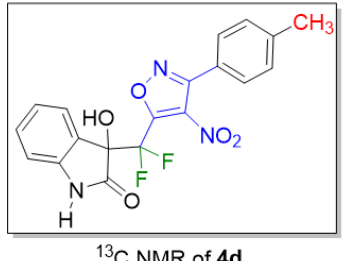

${ }^{13} \mathrm{C}$ NMR of $4 \mathrm{~d}$

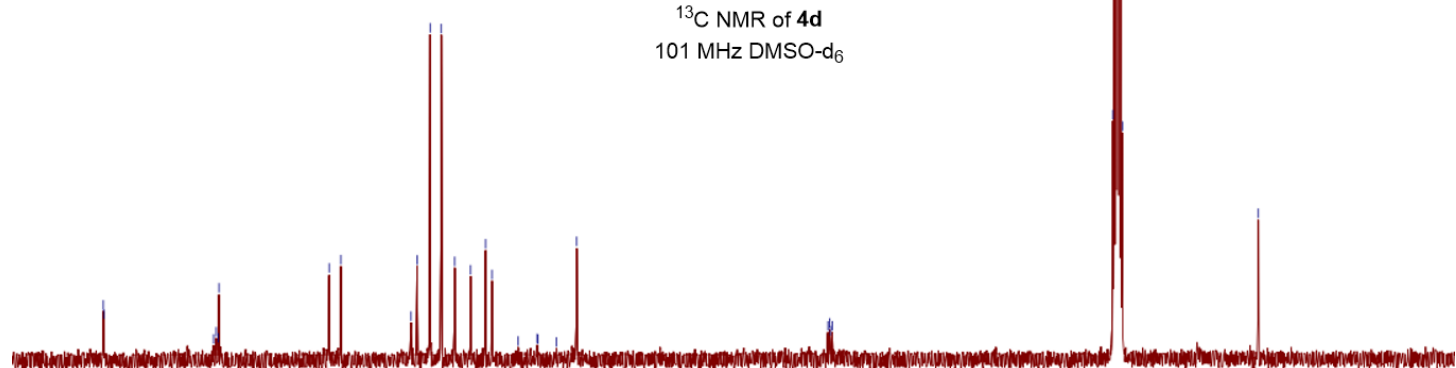

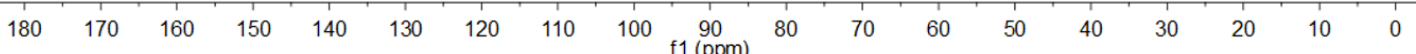


$-\operatorname{cog}_{0}$

응은응을

证
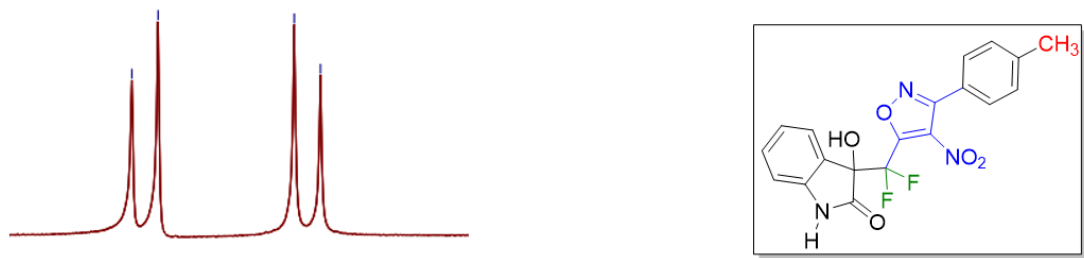

${ }^{19} \mathrm{~F}$ NMR of $\mathbf{4 d}$ $376 \mathrm{MHz}$ DMSO-d

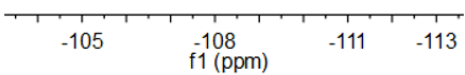

f1 (ppm)

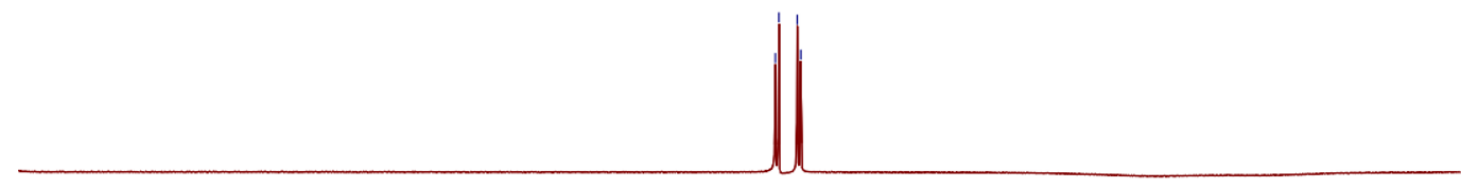

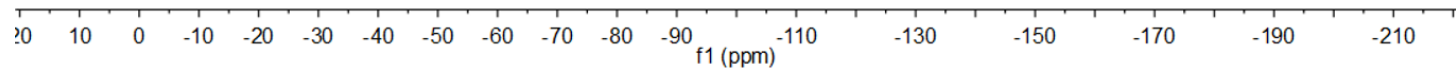
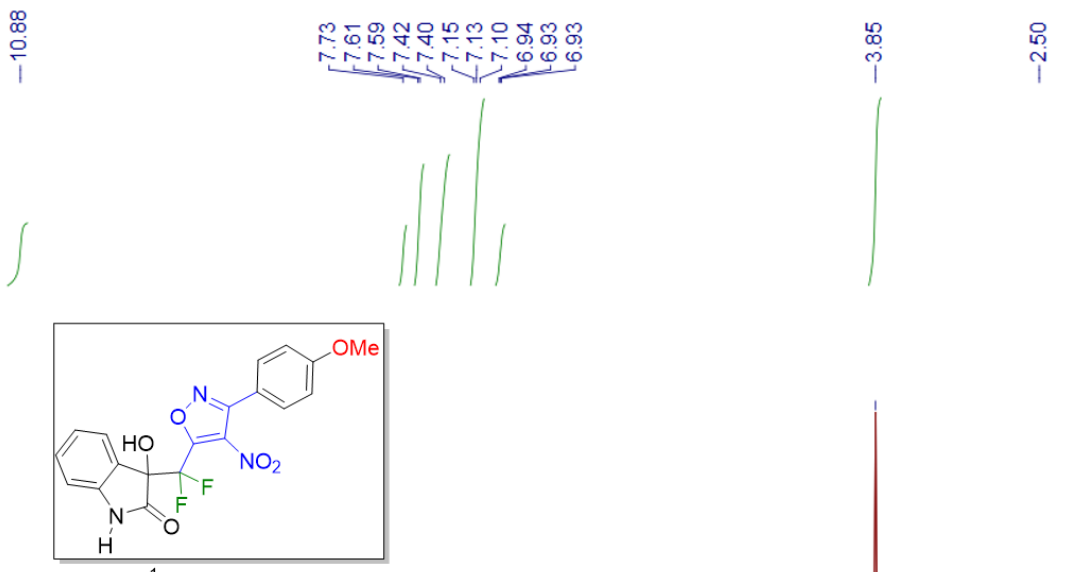

${ }^{1} \mathrm{H}$ NMR of $4 \mathrm{e}$

$400 \mathrm{MHz}$ DMSO-d 6

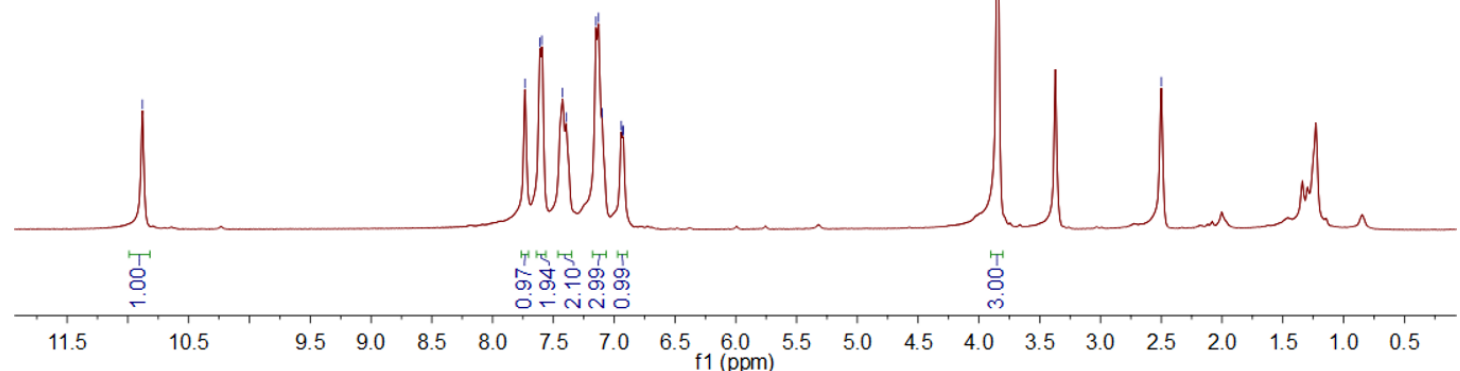




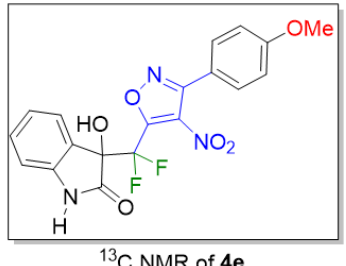

$101 \mathrm{MHz}$ DMSO-d

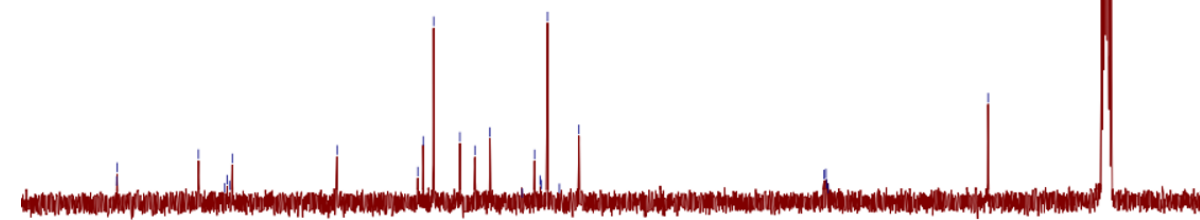

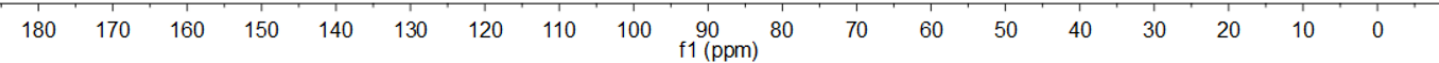

우ㅇㅛㅠ뭉

응 웅을

议
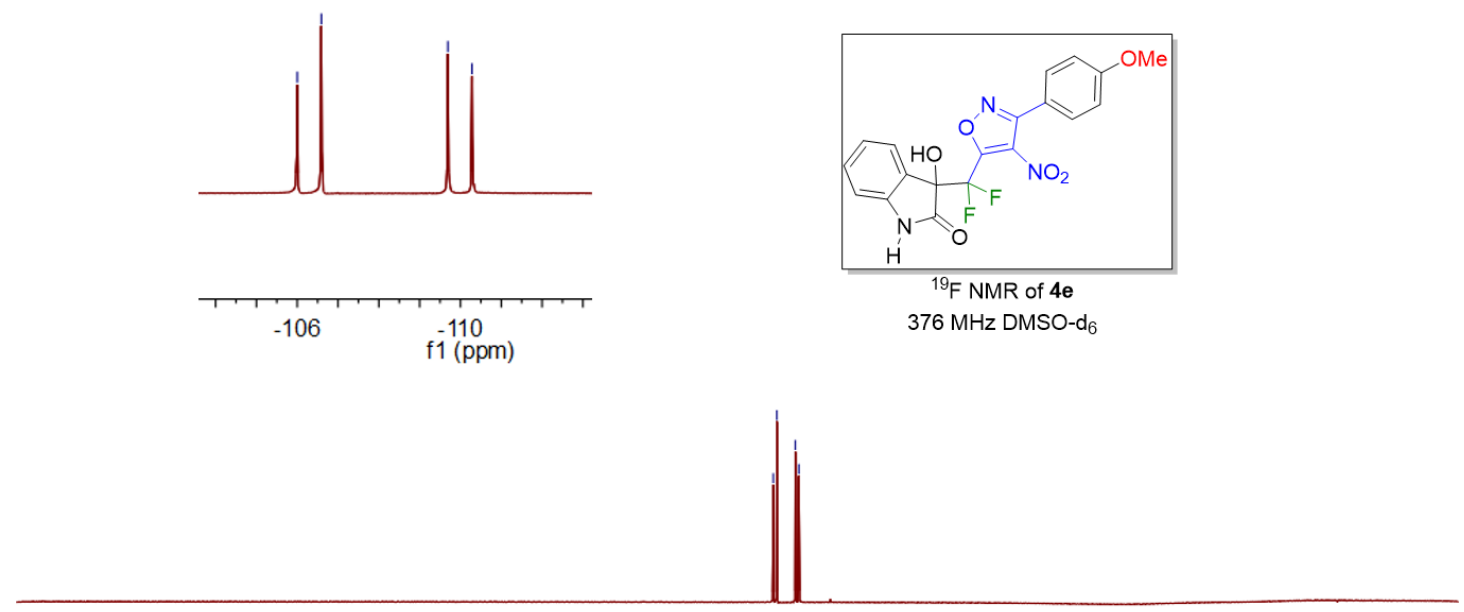

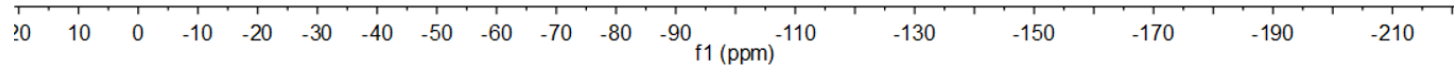




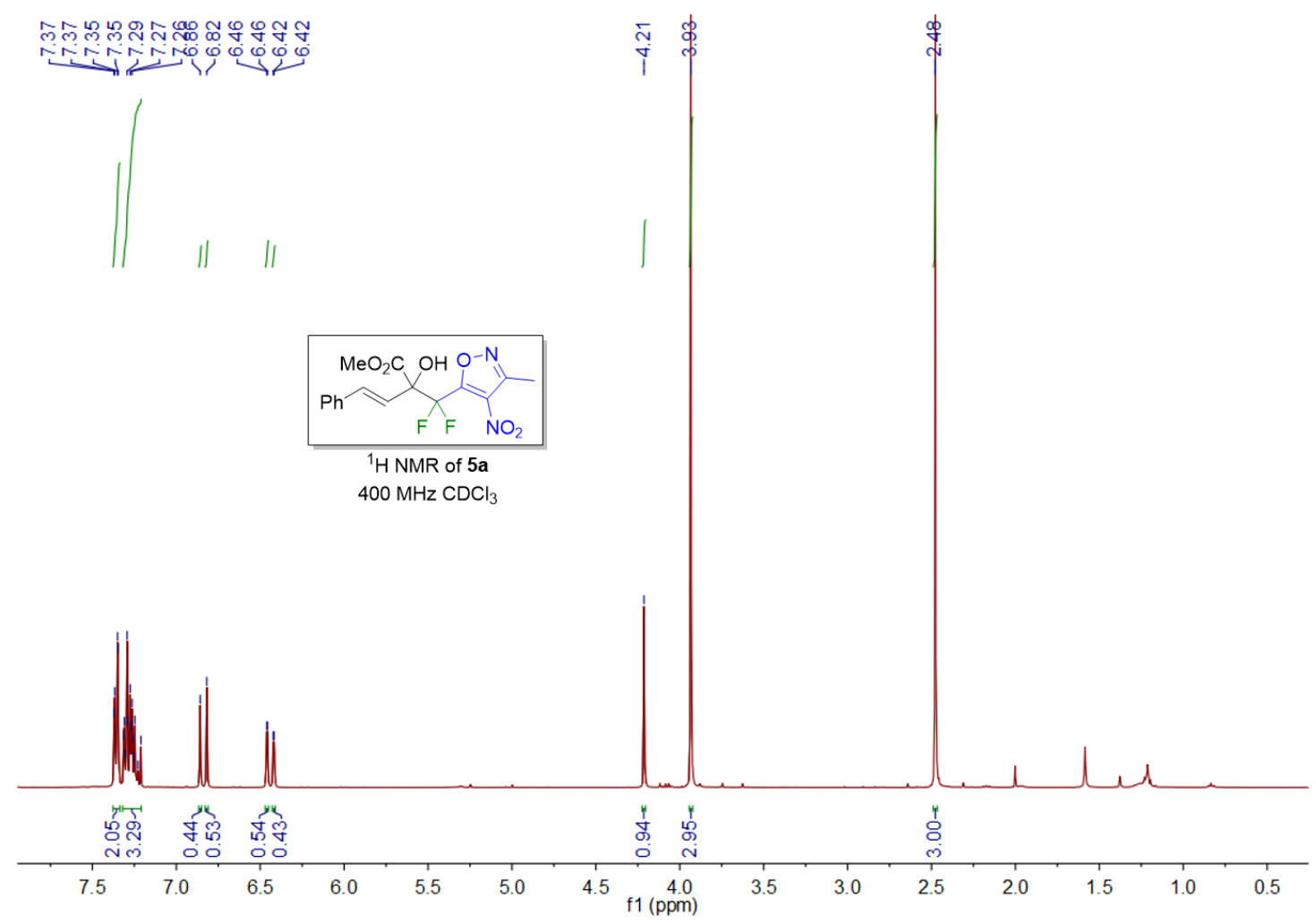

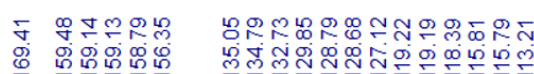

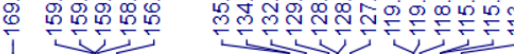
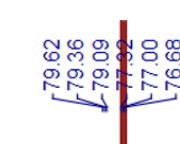

$\underset{\substack{1 \\ 0}}{\substack{0 \\ 0}}$

$\stackrel{ю}{\stackrel{1}{F}}$

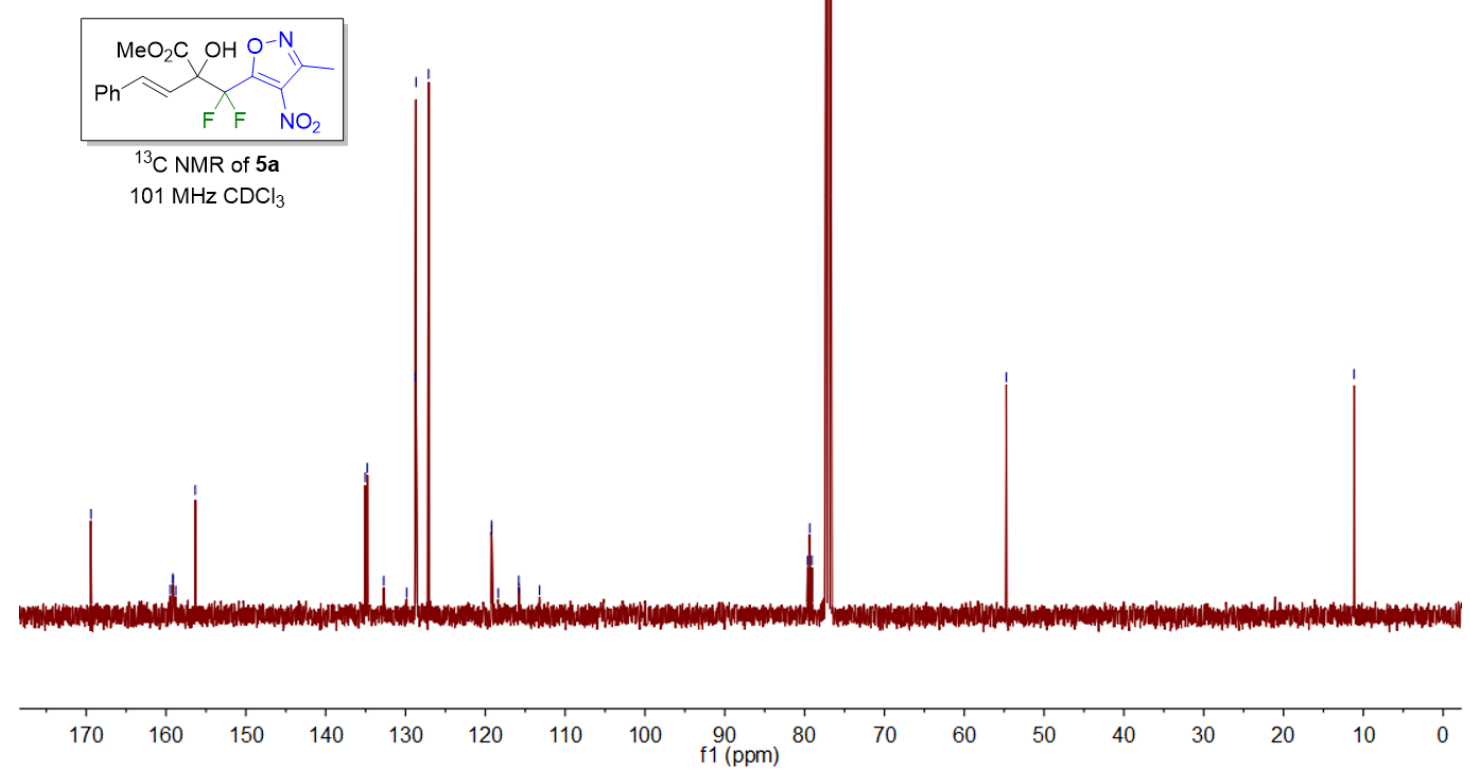



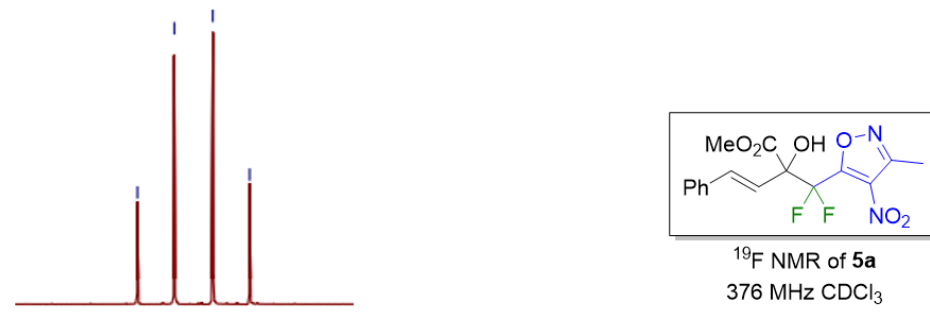

${ }^{19} \mathrm{~F}$ NMR of $5 \mathrm{a}$

$376 \mathrm{MHz} \mathrm{CDCl}_{3}$

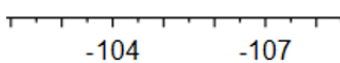

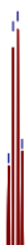

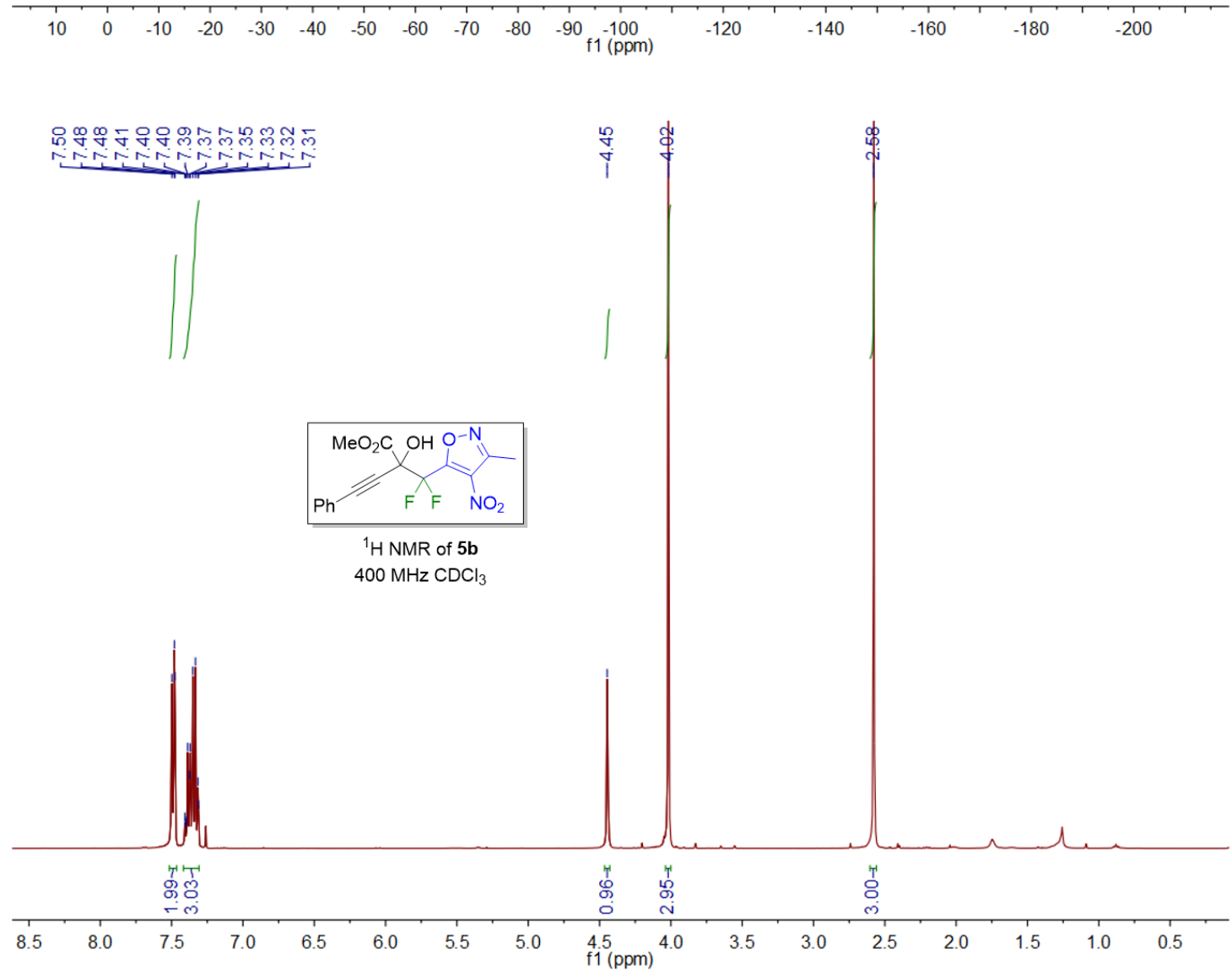



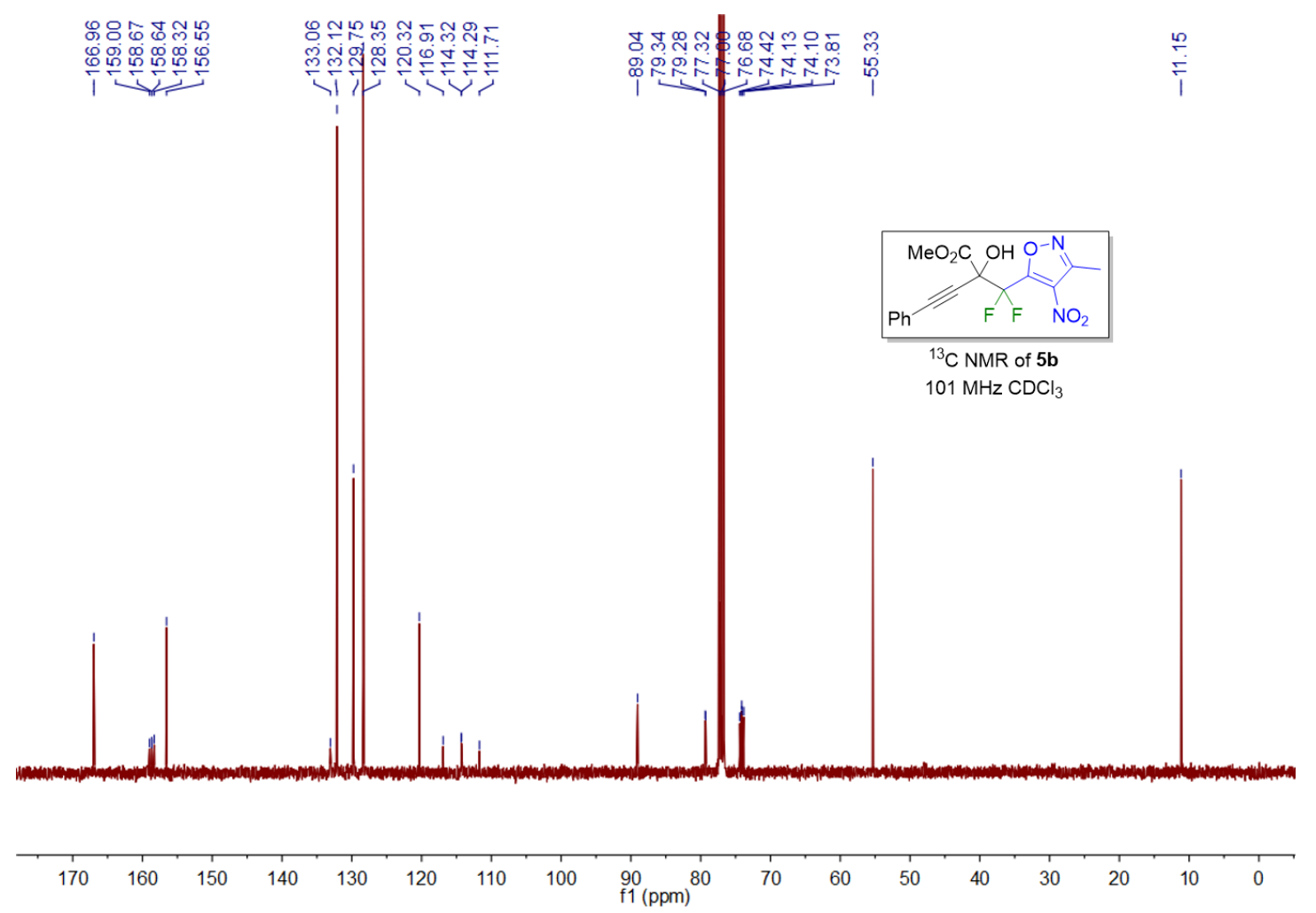

추유 ㄱ

응으융무

पi
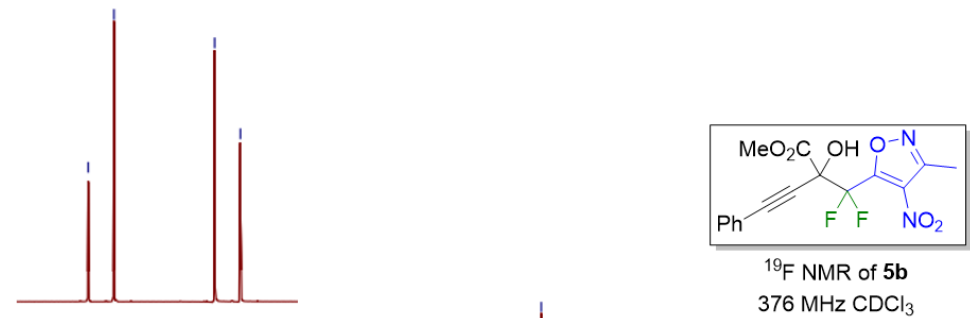

$\begin{array}{llll}-102 & -104 & -106 & -108\end{array}$

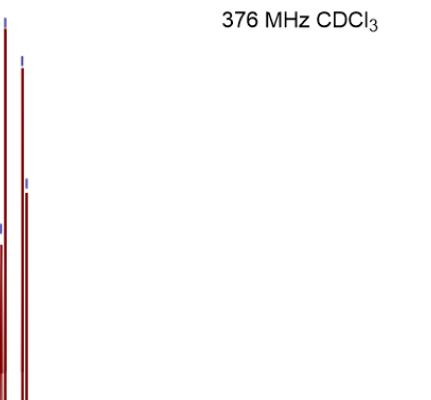

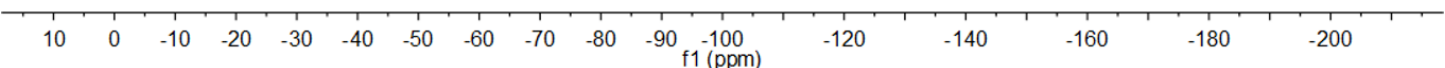




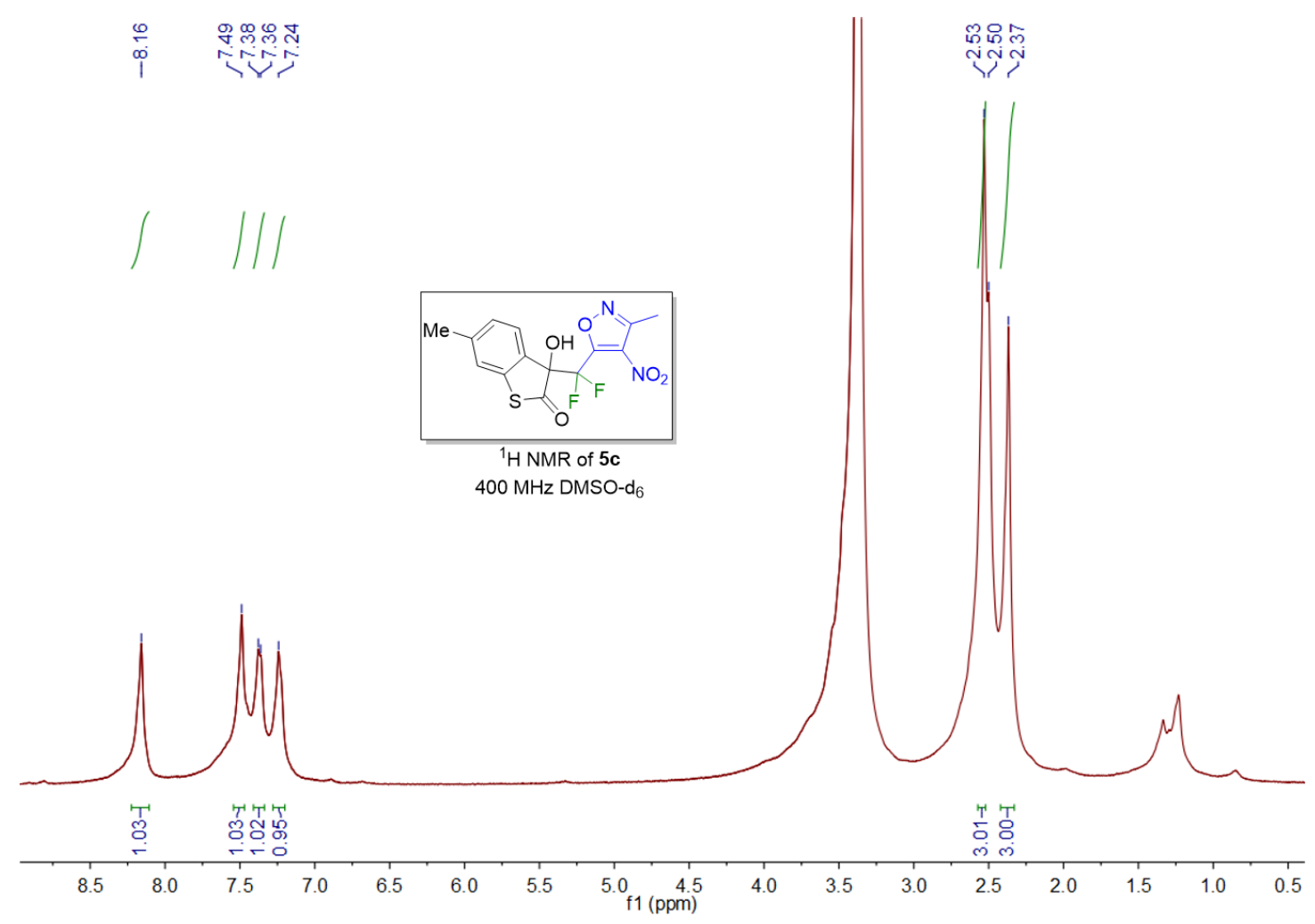

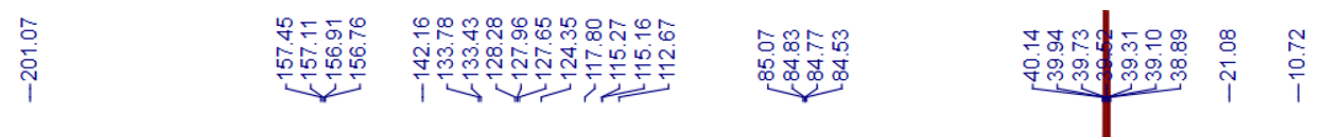

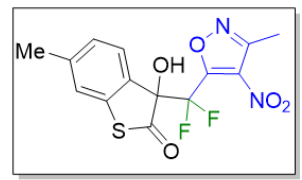

${ }^{13} \mathrm{C}$ NMR of $5 \mathrm{c}$

$101 \mathrm{MHz}$ DMSO-d

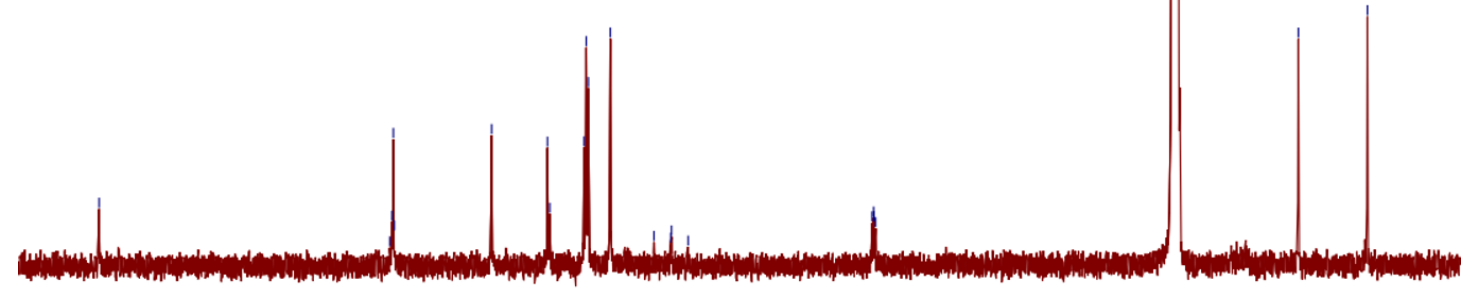

$\begin{array}{lllllllllllllllllllllllllll}1 & 1 & 200 & 190 & 180 & 170 & 160 & 150 & 140 & 130 & 120 & \begin{array}{c}110 \\ \mathrm{f} 1(\mathrm{ppm})\end{array} & 100 & 90 & 80 & 70 & 60 & 50 & 40 & 30 & 20 & 10 & 0\end{array}$ 

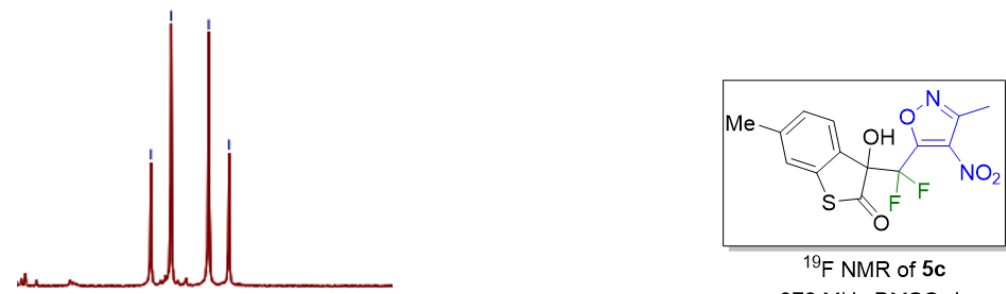

${ }^{19} \mathrm{~F}$ NMR of $5 \mathrm{c}$ $376 \mathrm{MHz}$ DMSO-d 6

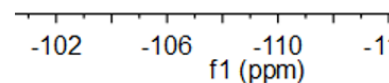

\begin{tabular}{llllllllllllllllll}
\hline 0 & 10 & 0 & -10 & -20 & -30 & -40 & -50 & -60 & -70 & -80 & -90 & -110 & -130 & -150 & -170 & -190 & -210
\end{tabular}

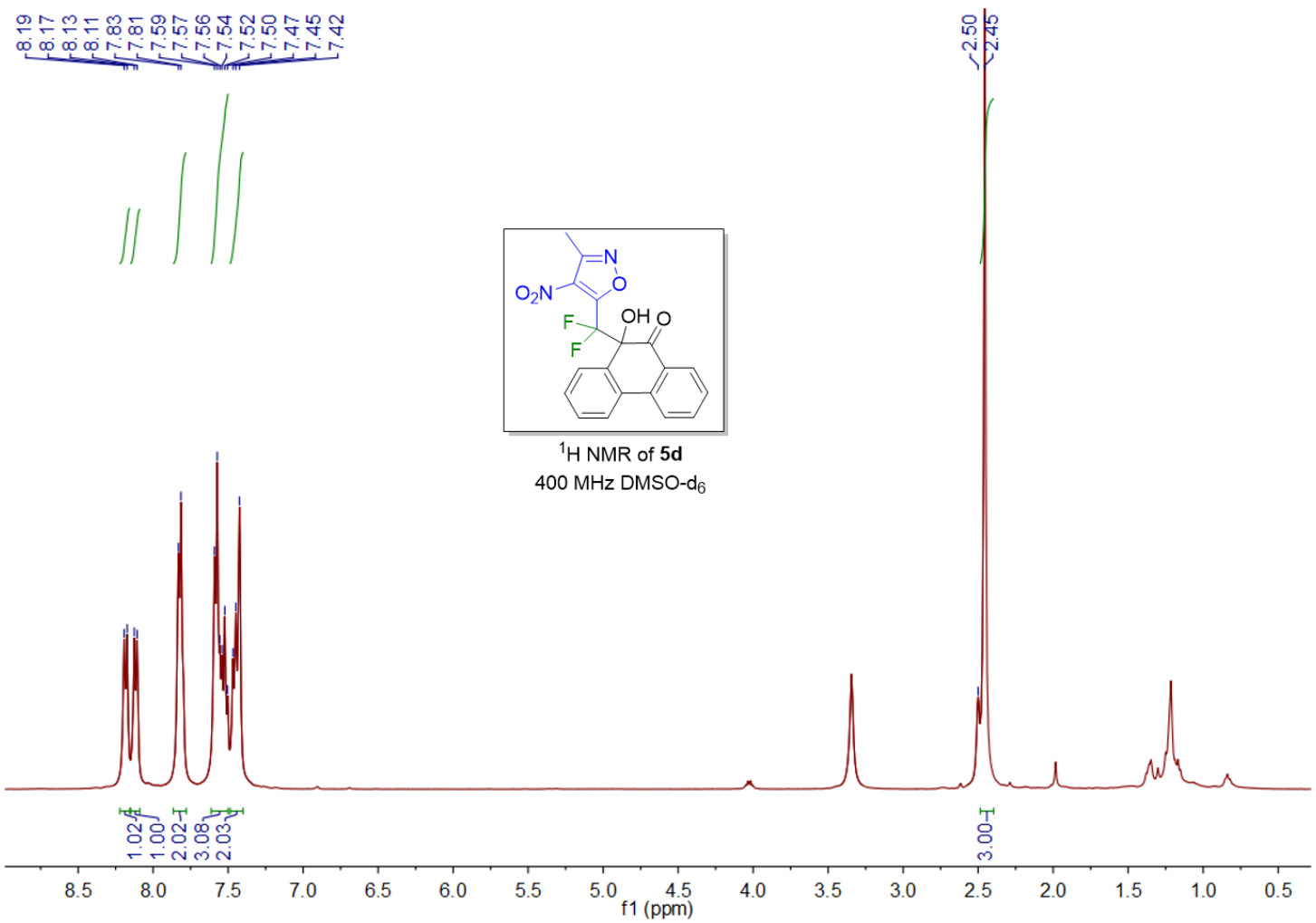



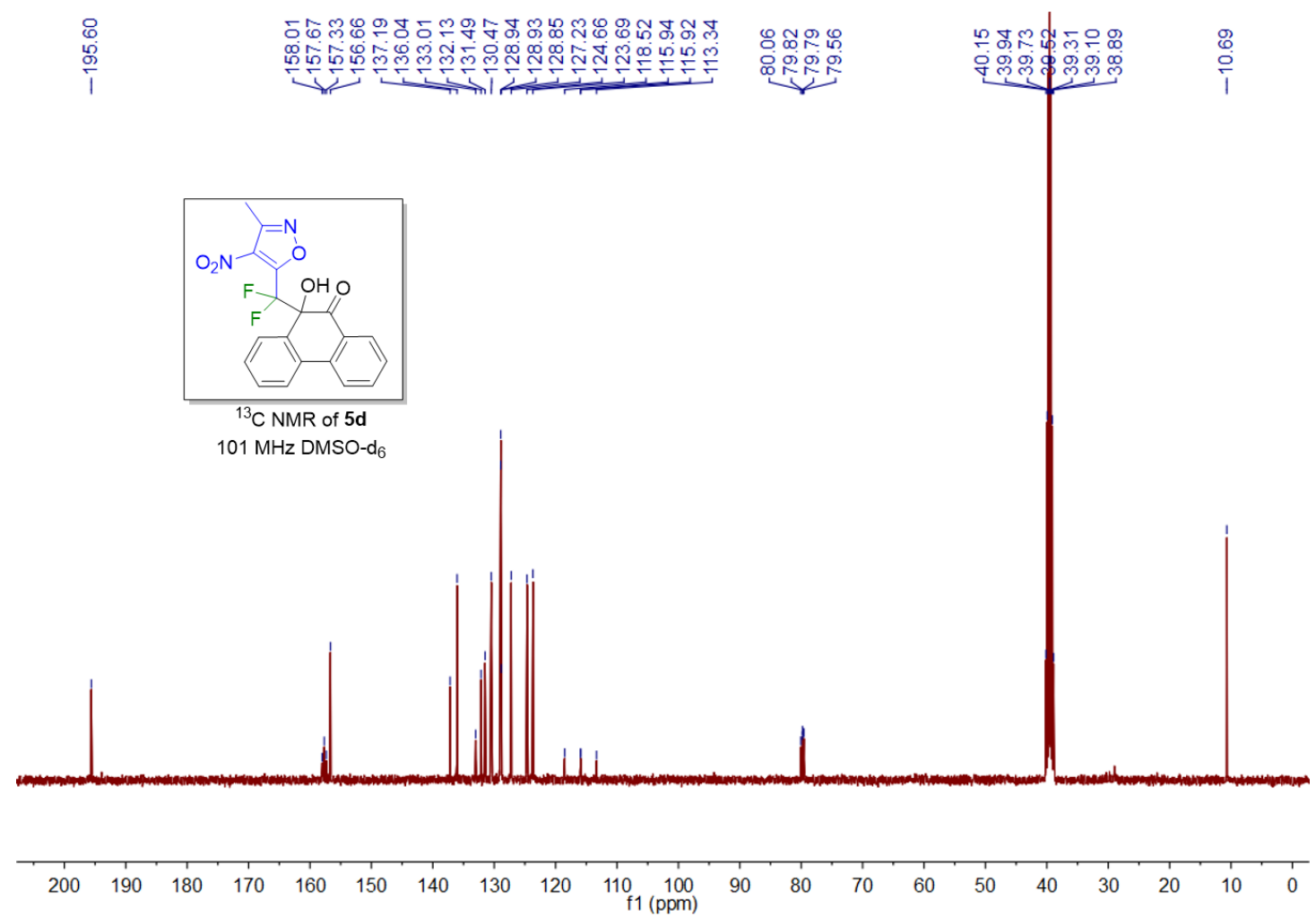

이요

잉훙뭉

운임
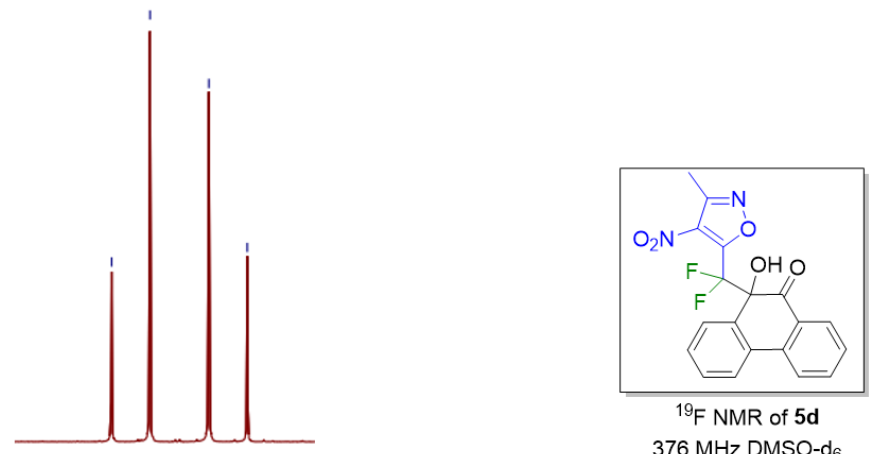

${ }^{19} \mathrm{~F}$ NMR of $5 \mathrm{~d}$

$376 \mathrm{MHz}$ DMSO-d
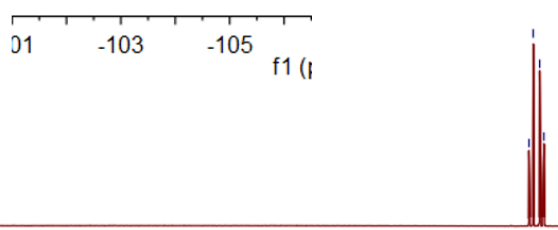

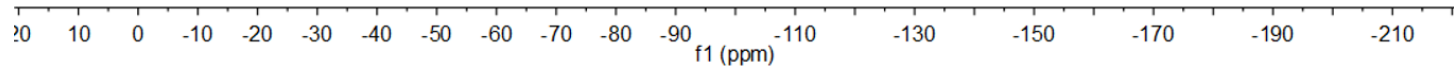



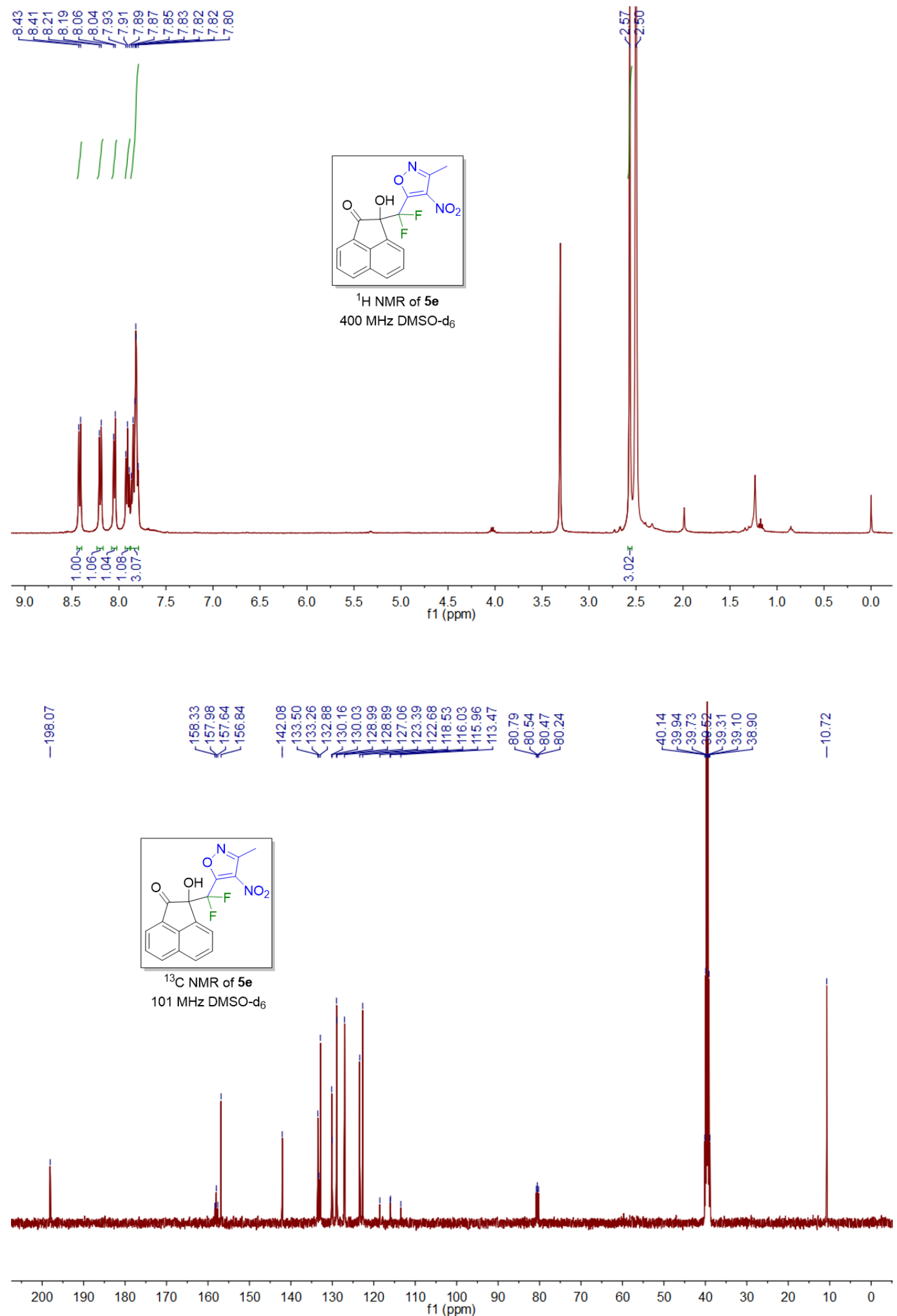


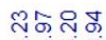

후ㅇㅝㅜ유융
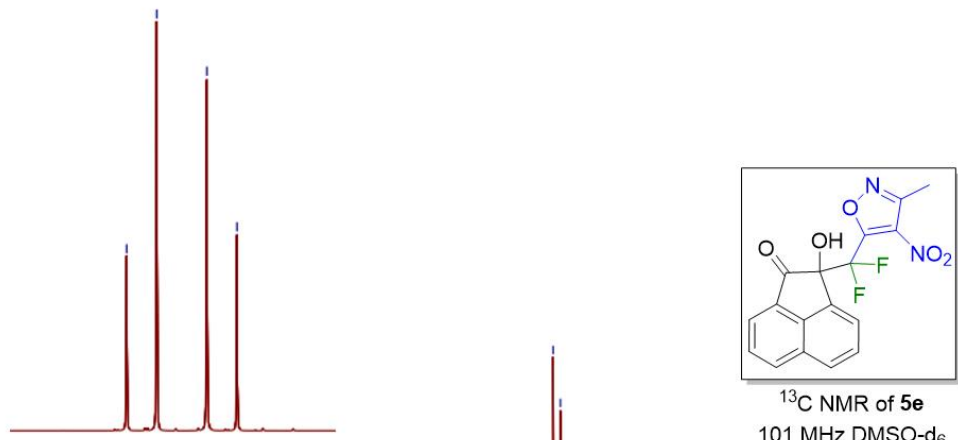

${ }^{13} \mathrm{C} \mathrm{NMR}$ of $5 \mathrm{e}$ $101 \mathrm{MHz}$ DMSO-d 6

\begin{tabular}{lllllllllllllllllllll}
\hline 0 & 10 & 0 & -10 & -20 & -30 & -40 & -50 & -60 & -70 & -80 & -90 & & 1 & 110 & -130 & -150 & -170 & -190 & -210
\end{tabular}

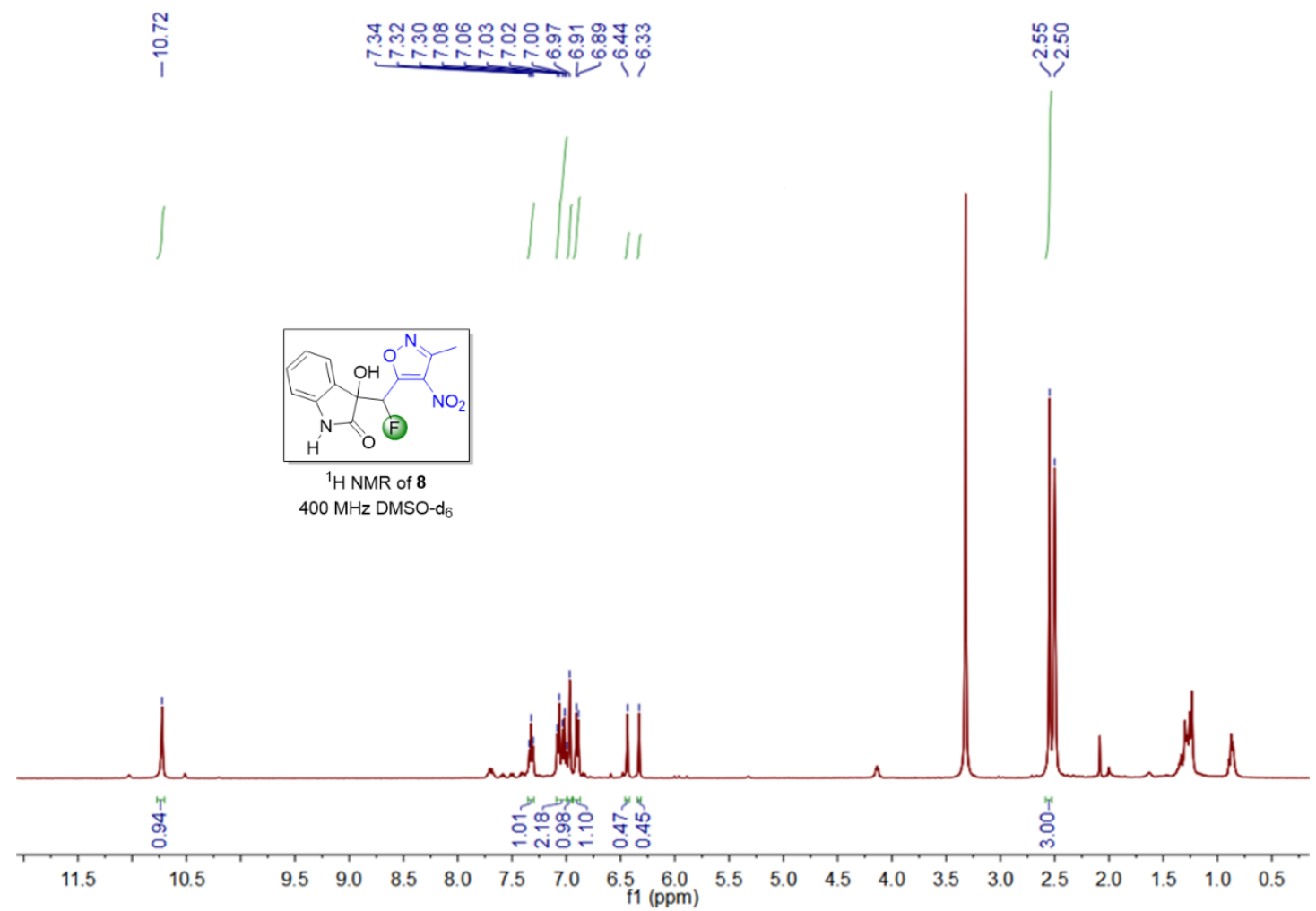

ผูํำ 

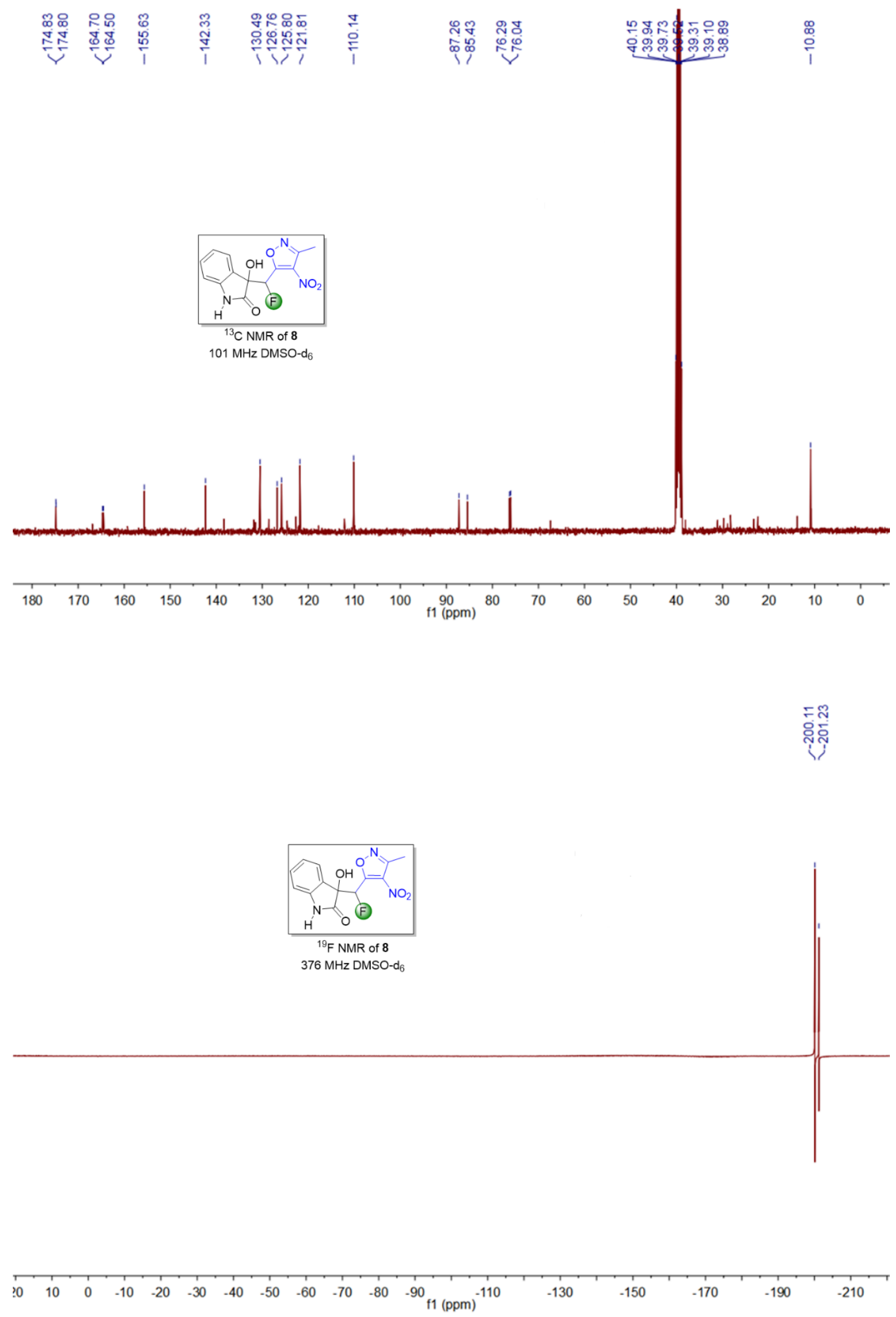


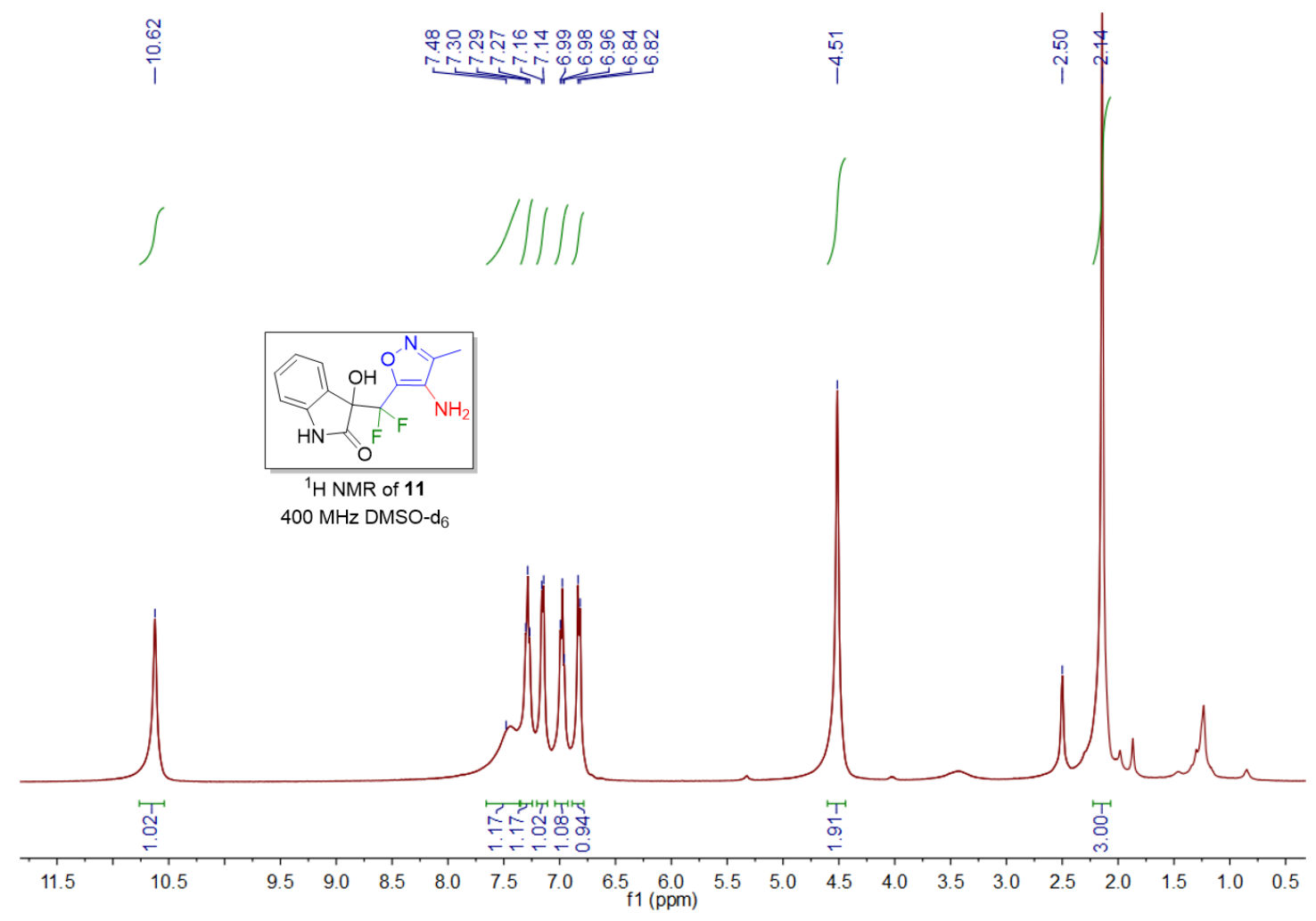

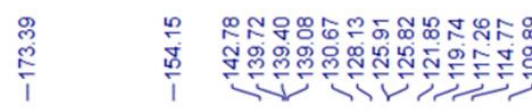

$\operatorname{con}$

산

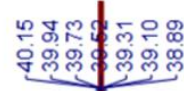

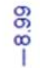

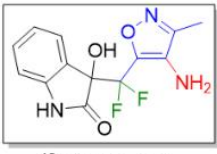

${ }^{13} \mathrm{C}$ NMR of 11

$101 \mathrm{MHz}$ DMSO-d

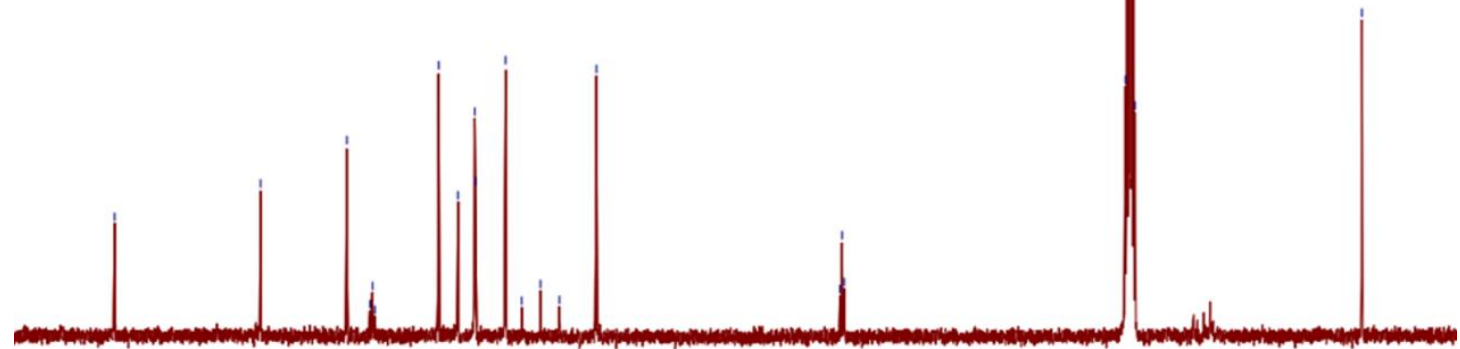

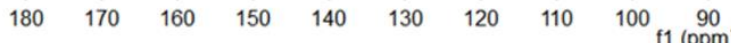

$80 \quad 70$

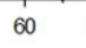

$50 \quad 40$

$10 \quad 0$ 


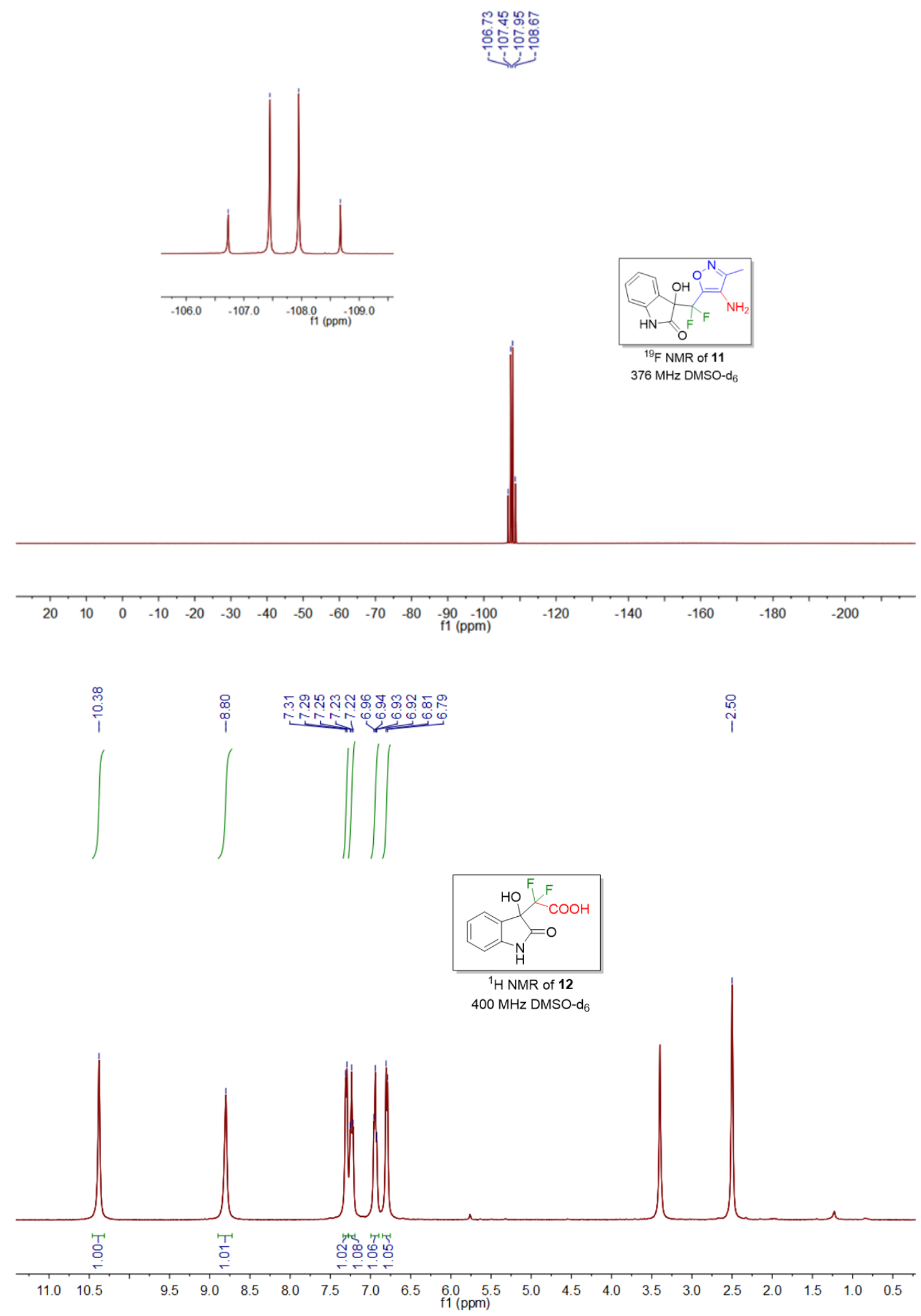




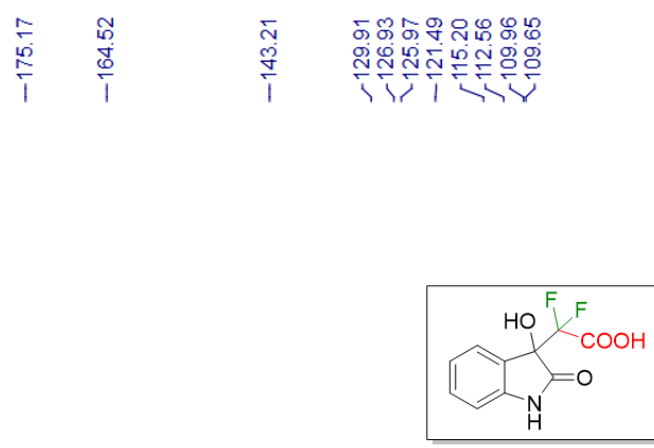

${ }^{13} \mathrm{C}$ NMR of 12

$101 \mathrm{MHz}$ DMSO-d 6

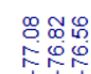

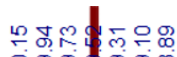

신

하에

$101 \mathrm{MHz}$ DMSO-do
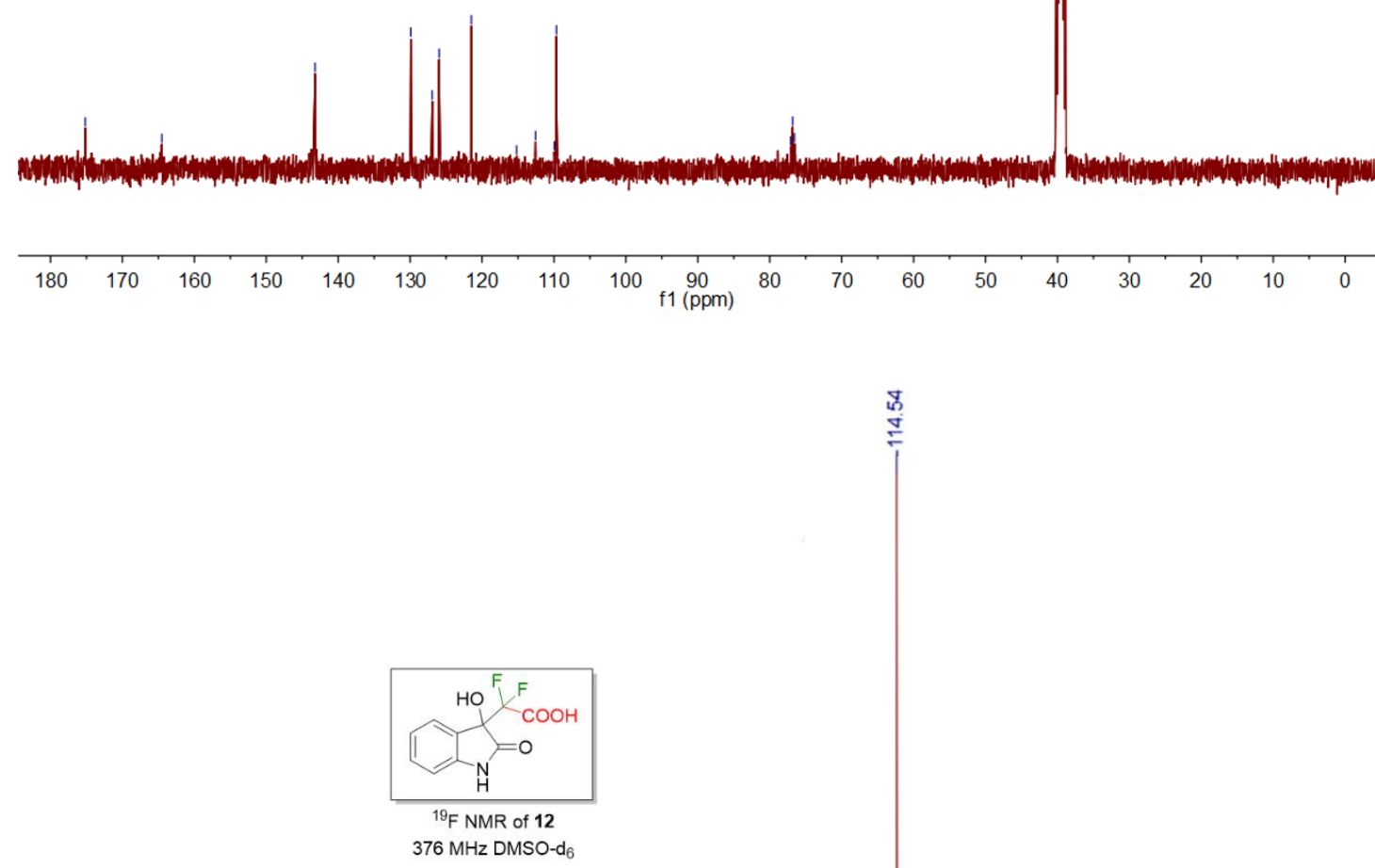

范

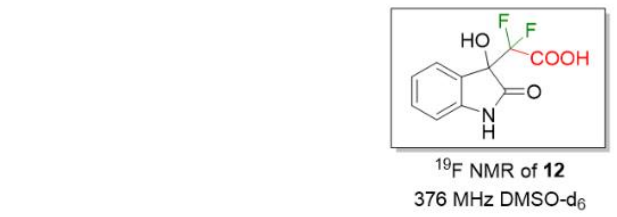

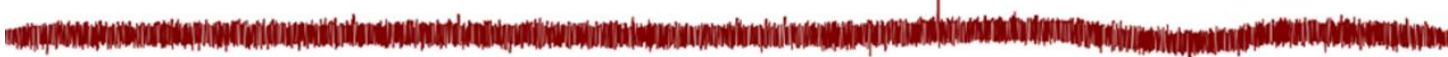

$\begin{array}{lllllllllllll}40 & 30 & 20 & 10 & 0 & -10 & -20 & -30 & -40 & -50 & -60 & -70 & -80 \\ \mathrm{f} 1(\mathrm{ppm}) & -90\end{array}$

$-110$

$-130$

$-150$

$170-190$ 